\title{
A Versatile NHC-Parent Silyliumylidene Cation for Catalytic Chemo- and Regioselective Hydroboration
}

Bi-Xiang Leong, ${ }^{\text {a Jiawen Lee, }}{ }^{\mathrm{a}}$ Yan Li, ${ }^{\mathrm{a}}$ Ming-Chung Yang, ${ }^{\mathrm{b}}$ Chi-Kit Siu, ${ }^{* \mathrm{~d}}$ Ming-Der $\mathrm{Su}^{* \mathrm{~b}, \mathrm{c}}$ and Cheuk-Wai So ${ }^{* a}$

aDivision of Chemistry and Biological Chemistry, School of Physical and Mathematical Sciences, Nanyang Technological University, 637371 Singapore. ${ }^{b}$ Department of Applied Chemistry, National Chiayi University, Chiayi 60004, Taiwan. ${ }^{\mathrm{c}}$ Department of Medicinal and Applied Chemistry, Kaohsiung Medical University, Kaohsiung 80708, Taiwan. ${ }^{\mathrm{d}}$ Department of Chemistry, City University of Hong Kong, Kowloon Tong, Hong Kong SAR, China.

E-mail: CWSo@ntu.edu.sg

\section{Supporting Information}

\section{Content:}

S1. Experimental sections

S2. Selected spectra

S3. X-ray data collection and structural refinement

S4. Theoretical studies

S5. References 


\section{S1. Experimental sections}

General Procedures. All manipulations were carried out under an inert atmosphere of argon gas by standard Schlenk techniques. Compound $\mathbf{1}$ was prepared according to the literature procedure. ${ }^{\mathrm{S} 1}$ Toluene was dried over $\mathrm{Na} / \mathrm{K}$ alloy and distilled prior to use. $\mathrm{C}_{6} \mathrm{D}_{6}$ and $d_{5}$-pyridine were dried over $\mathrm{K}$ metal and distilled prior to use. $\mathrm{CH}_{2} \mathrm{Cl}_{2}$ and $\mathrm{CDCl}_{3}$ were dried over $\mathrm{CaH}_{2}$ and distilled prior to use. Chemicals were purchased and used directly without further purification. The ${ }^{1} \mathrm{H},{ }^{11} \mathrm{~B},{ }^{11} \mathrm{~B}\left\{{ }^{1} \mathrm{H}\right\},{ }^{13} \mathrm{C}\left\{{ }^{1} \mathrm{H}\right\},{ }^{29} \mathrm{Si}$ and ${ }^{29} \mathrm{Si}\left\{{ }^{1} \mathrm{H}\right\}$ NMR spectra were recorded on a JEOL ECA 400 spectrometer. The NMR spectra were recorded in $\mathrm{C}_{6} \mathrm{D}_{6}, \mathrm{CDCl}_{3}$ or $d_{5}$-pyridine, and the chemical shifts are relative to $\mathrm{SiMe}_{4}$ for ${ }^{1} \mathrm{H},{ }^{13} \mathrm{C}$ and ${ }^{29} \mathrm{Si} ; \mathrm{BF}_{3} \cdot \mathrm{Et}_{2} \mathrm{O}$ for ${ }^{11} \mathrm{~B}$, respectively. The following abbreviations are used to describe signal multiplicities: $\mathrm{s}=$ singlet, $\mathrm{d}=$ doublet, $\mathrm{t}=$ triplet, $\mathrm{q}=$ quartet and $\mathrm{m}=$ multiplet. Coupling constants $J$ are given in Hertz $(\mathrm{Hz})$. Melting points were measured in sealed glass tubes and were not corrected. Electrospray ionization (ESI) mass spectra were obtained at the Mass Spectrometry Laboratory at the Division of Chemistry and Biological Chemistry, Nanyang Technological University.

\section{Synthesis of compound 3}

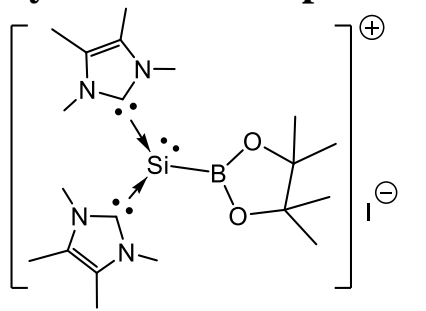

Compound 1 ( $4.0 \mathrm{mg}, 0.01 \mathrm{mmol})$ and pinacolborane, HBpin $(14.5 \mu \mathrm{L}, 10$ equiv, $0.10 \mathrm{mmol})$ were mixed with $0.5 \mathrm{~mL}$ of $\mathrm{C}_{6} \mathrm{D}_{6}$ in a J-Young NMR tube at room temperature. The resulting mixture was stirred at $60{ }^{\circ} \mathrm{C}$ for $5 \mathrm{~min}$. Volatiles were immediately removed at $60{ }^{\circ} \mathrm{C}$ in vacuo. The residue was extracted with benzene $(1 \mathrm{~mL})$. After filtration, the filtrate was removed in vacuo to afford compound $\mathbf{3}$ as a colorless solid (1.6 mg, Yield: $30 \%)$. When the reaction was performed for 16 hours, the yield of compound 3 is $63 \%$ (3.3 mg). Mp: $167{ }^{\circ} \mathrm{C}$ (dec.). HRMS (ESI): $\mathrm{m} / \mathrm{z}$ calcd for $\mathrm{C}_{20} \mathrm{H}_{37} \mathrm{BIN}_{4} \mathrm{O}_{2} \mathrm{Si}[\mathrm{M}-\mathrm{H}]^{+}$: 531.18236; found: 531.18240. Satisfactory elemental analysis data could not be obtained due to compound 3 being highly sensitive to moisture and air. ${ }^{1} \mathrm{H}$ NMR $\left(395.9 \mathrm{MHz}, 24{ }^{\circ} \mathrm{C}, \mathrm{C}_{6} \mathrm{D}_{6}, \mathrm{ppm}\right): \delta=3.69\left(\mathrm{~s}, 12 \mathrm{H}, \mathrm{N}-\mathrm{CH}_{3}\right), 1.75$ (s, $\left.12 \mathrm{H}, \mathrm{C}-\mathrm{CH}_{3}\right), 1.08$ (s, $\left.12 \mathrm{H}, \mathrm{Bpin}-\mathrm{CH}_{3}\right) .{ }^{11} \mathrm{~B}$ NMR $\left(128.41 \mathrm{MHz}, 24{ }^{\circ} \mathrm{C}, \mathrm{C}_{6} \mathrm{D}_{6}, \mathrm{ppm}\right): \delta=0.92$. ${ }^{11} \mathrm{~B}\left\{{ }^{1} \mathrm{H}\right\}$ NMR $\left(128.41 \mathrm{MHz}, 24{ }^{\circ} \mathrm{C}, \mathrm{C}_{6} \mathrm{D}_{6}, \mathrm{ppm}\right): \delta=0.95 .{ }^{29} \mathrm{Si} \mathrm{NMR}\left(78.65 \mathrm{MHz}, 24{ }^{\circ} \mathrm{C}, \mathrm{C}_{6} \mathrm{D}_{6}\right.$, ppm): $\delta=-93.0 .{ }^{29} \mathrm{Si}\left\{{ }^{1} \mathrm{H}\right\} \mathrm{NMR}\left(78.65 \mathrm{MHz}, 24{ }^{\circ} \mathrm{C}, \mathrm{C}_{6} \mathrm{D}_{6}, \mathrm{ppm}\right): \delta=-95.6$. The Si NMR signal is relatively weak due to quadrupolar broadening with the boron nucleus $(I=3 / 2)$.

\section{Decomposition of compound 3}

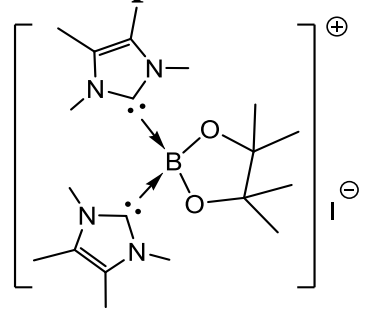

Prolonged crystallization of $\mathbf{3}$ in $\mathrm{C}_{6} \mathrm{D}_{6}$ or toluene afforded a mixture of white precipitates $\left[\left(\mathrm{I}_{\mathrm{Me}}\right)_{2}\right.$ Bpin]I and $\left[\mathrm{I}_{\mathrm{Me}}-\mathrm{H}\right] \mathrm{I}$. They are insoluble in $\mathrm{C}_{6} \mathrm{D}_{6}$ but are highly soluble in $\mathrm{CDCl}_{3}$. Colorless crystals of $\left[\left(\mathrm{I}_{\mathrm{Me}}\right)_{2} \mathrm{Bpin}\right] \mathrm{I}$ and $\left[\mathrm{I}_{\mathrm{Me}}-\mathrm{H}\right] \mathrm{I}$ were isolated from their concentrated $\mathrm{CH}_{2} \mathrm{Cl}_{2}$ solution. ${ }^{1} \mathrm{H}$ NMR of $\left[\left(\mathrm{I}_{\mathrm{Me}}\right)_{2} \mathrm{Bpin}\right] \mathrm{I}\left(395.9 \mathrm{MHz}, 24{ }^{\circ} \mathrm{C}, \mathrm{CDCl}_{3}, \mathrm{ppm}\right): \delta=3.89(\mathrm{~s}, 12 \mathrm{H}, \mathrm{N}-$ 
$\left.\mathrm{CH}_{3}\right), 2.22\left(\mathrm{~s}, 12 \mathrm{H}, \mathrm{C}-\mathrm{CH}_{3}\right), 0.14\left(\mathrm{~s}, 12 \mathrm{H}, \mathrm{Bpin}-\mathrm{CH}_{3}\right) .{ }^{11} \mathrm{~B} \mathrm{NMR}$ of $\left[\left(\mathrm{I}_{\mathrm{Me}}\right)_{2} \mathrm{Bpin}\right] \mathrm{I}(128.41 \mathrm{MHz}$, $\left.24{ }^{\circ} \mathrm{C}, \mathrm{CDCl}_{3}, \mathrm{ppm}\right): \delta=2.37 .{ }^{11} \mathrm{~B}\left\{{ }^{1} \mathrm{H}\right\} \mathrm{NMR}\left(128.41 \mathrm{MHz}, 24{ }^{\circ} \mathrm{C}, \mathrm{CDCl}_{3}, \mathrm{ppm}\right): \delta=2.56$.

\section{Formation of Int01}

Compound 1 ( $4.0 \mathrm{mg}, 0.01 \mathrm{mmol}$ ) and $0.5 \mathrm{~mL}$ of $d_{5}$-pyridine were mixed in a J-Young NMR tube. The NMR tube was immersed in liquid nitrogen under vacuum to afford a frozen solution. The J-Young NMR tube was lifted from liquid nitrogen and 1 bar of $\mathrm{CO}_{2}$ gas was then added. The reaction mixture was warmed to $-40^{\circ} \mathrm{C}$ and analysed by NMR spectroscopy. ${ }^{1} \mathrm{H}$ NMR (395.9 MHz, $-40{ }^{\circ} \mathrm{C}, d_{5}$-pyridine, ppm): $\delta=9.99(\mathrm{~s}, 1 \mathrm{H}, \mathrm{Si}-H), 3.71\left(\mathrm{~s}, 12 \mathrm{H}, \mathrm{N}-\mathrm{CH}_{3}\right), 1.97$ (s, $\left.12 \mathrm{H}, \mathrm{C}-\mathrm{CH}_{3}\right) .{ }^{29} \mathrm{Si} \mathrm{NMR}\left(160.5 \mathrm{MHz},-40{ }^{\circ} \mathrm{C}, d_{5}\right.$-pyridine, ppm): $\delta-68.9\left({ }^{1} J_{\mathrm{SiH}}=202 \mathrm{~Hz}\right)$. ${ }^{29} \mathrm{Si}\left\{{ }^{1} \mathrm{H}\right\} \mathrm{NMR}\left(78.65 \mathrm{MHz},-40{ }^{\circ} \mathrm{C}, d_{5}\right.$-pyridine, $\left.\mathrm{ppm}\right): \delta=-66.2$.

\section{General procedures for the catalytic hydroboration of carbon dioxide using 1 as the catalyst:}

\section{Synthesis of compound $2 a-2 c$}

Catalyst 1 (4.0 mg, $0.01 \mathrm{mmol})$, internal standard 1,3,5-trimethoxybenzene $(8.4 \mathrm{mg}, 0.05 \mathrm{mmol}$, 5 equiv.) and $0.5 \mathrm{~mL}$ of $\mathrm{C}_{6} \mathrm{D}_{6}$ were mixed in a J-Young NMR tube. Pinacolborane, HBpin (14.5 $\mu \mathrm{L}, 0.10 \mathrm{mmol}, 10$ equiv.) was then added. The NMR tube was immersed in liquid nitrogen under vacuum to obtain a frozen solution. The J-Young NMR tube was lifted from liquid nitrogen and 1 bar of $\mathrm{CO}_{2}$ gas was then added. The reaction mixture was warmed to room temperature and then heated at $90{ }^{\circ} \mathrm{C}$. The reaction was monitored by NMR spectroscopy. The yields of products were reported according to the integration of ${ }^{1} \mathrm{H}$ NMR signals of $\operatorname{pinBOC}(=\mathrm{O}) \mathrm{H}(\mathbf{2 a})$ at $0.93 \mathrm{ppm}$ and $(\operatorname{pinB})_{2} \mathrm{O}(\mathbf{2 c})$ at $1.02 \mathrm{ppm}$ with reference to the $-\mathrm{OMe}$ and $\mathrm{C}_{\mathrm{Ar}}-H$ protons $(3.36,6.20 \mathrm{ppm})$ of the internal standard, 1,3,5-trimethoxybenzene. The chemical shifts of the products agree with the reported values in the literature and independent in-situ NMR-scale syntheses. ${ }^{\text {S2 }}$ Formation of $\mathbf{2 b}$ was not observed throughout the catalytic reaction. The catalytic studies were repeated thrice.

\section{Characterization of compound 2a}<smiles>[B][B]OC=O</smiles>

${ }^{1} \mathrm{H}$ NMR $\left(395.9 \mathrm{MHz}, 24{ }^{\circ} \mathrm{C}, \mathrm{C}_{6} \mathrm{D}_{6}, \mathrm{ppm}\right): \delta=8.15$ (s, $\left.1 \mathrm{H}, \mathrm{C}-\mathrm{H}\right), 0.93$ (s, 12H, Bpin-C $\left.\mathrm{H}_{3}\right) .{ }^{11} \mathrm{~B}$ NMR (128.41 MHz, $\left.24{ }^{\circ} \mathrm{C}, \mathrm{C}_{6} \mathrm{D}_{6}, \mathrm{ppm}\right): \delta=22.0 .{ }^{11} \mathrm{~B}\left\{{ }^{1} \mathrm{H}\right\} \mathrm{NMR}\left(128.41 \mathrm{MHz}, 24{ }^{\circ} \mathrm{C}, \mathrm{C}_{6} \mathrm{D}_{6}\right.$, ppm): $\delta=22.0 .{ }^{13} \mathrm{C}\left\{{ }^{1} \mathrm{H}\right\}$ NMR $\left(99.5 \mathrm{MHz}, 24{ }^{\circ} \mathrm{C}, \mathrm{C}_{6} \mathrm{D}_{6}, \mathrm{ppm}\right): \delta=157.7\left(\mathrm{~s}, \mathrm{O}_{2} \mathrm{CH}\right), 93.5(\mathrm{~s}$, $\mathrm{CMe}), 24.2$ (s, CMe).

\section{Characterization of compound $2 c$}<smiles>[B]O[B]O[Pb]</smiles>

${ }^{1} \mathrm{H}$ NMR $\left(395.9 \mathrm{MHz}, 24{ }^{\circ} \mathrm{C}, \mathrm{C}_{6} \mathrm{D}_{6}, \mathrm{ppm}\right): \delta=1.02$ (s, 24H, Bpin-CH$) .{ }^{11} \mathrm{~B}$ NMR $(128.41$ $\left.\mathrm{MHz}, 24{ }^{\circ} \mathrm{C}, \mathrm{C}_{6} \mathrm{D}_{6}, \mathrm{ppm}\right): \delta=20.9 .{ }^{11} \mathrm{~B}\left\{{ }^{1} \mathrm{H}\right\} \mathrm{NMR}\left(128.41 \mathrm{MHz}, 24{ }^{\circ} \mathrm{C}, \mathrm{C}_{6} \mathrm{D}_{6}, \mathrm{ppm}\right): \delta=20.9$. ${ }^{13} \mathrm{C}\left\{{ }^{1} \mathrm{H}\right\}$ NMR $\left(99.5 \mathrm{MHz}, 24{ }^{\circ} \mathrm{C}, \mathrm{C}_{6} \mathrm{D}_{6}, \mathrm{ppm}\right): \delta=84.4$ (s, CMe), 24.5 (s, CMe).

\section{Synthesis of compound $2 d-2 e$}

Catalyst 1 (4.0 mg, $0.01 \mathrm{mmol})$, internal standard methyltriphenylsilane (27.4 mg, $0.10 \mathrm{mmol}$, 10 equiv.) and $0.5 \mathrm{~mL}$ of $\mathrm{C}_{6} \mathrm{D}_{6}$ were mixed in a J-Young NMR tube. Borane dimethylsulfide, 
$\mathrm{BH}_{3} . \mathrm{SMe}_{2}(9.5 \mu \mathrm{L}, 0.10 \mathrm{mmol}, 10$ equiv. $)$ was then added. The NMR tube was immersed in liquid nitrogen under vacuum to obtain a frozen solution. The J-Young NMR tube was lifted from liquid nitrogen and 1 bar of $\mathrm{CO}_{2}$ gas was then added. The reaction mixture was warmed to room temperature and then heated at $90{ }^{\circ} \mathrm{C}$. The reaction was monitored by NMR spectroscopy. The yields of products were reported according to the integration of ${ }^{1} \mathrm{H}$ NMR signals of $\mathrm{B}(\mathrm{OMe})_{3}(\mathbf{2 d})$ at $3.42 \mathrm{ppm}$ and $(\mathrm{MeOBO})_{3}(\mathbf{2 e})$ at $3.34 \mathrm{ppm}$ with reference to the Me protons $(0.71 \mathrm{ppm})$ of the internal standard, methyltriphenylsilane. The chemical shifts of the products agree with the reported values in the literature and independent in-situ NMR-scale syntheses. ${ }^{\mathrm{S} 3}$ The catalytic studies were repeated thrice. Blank reaction of $\mathrm{BH}_{3} . \mathrm{SMe}_{2}$ and $\mathrm{Ph}_{3} \mathrm{SiMe}$ was conducted $\left(90^{\circ} \mathrm{C}, 30 \mathrm{~min}, \mathrm{C}_{6} \mathrm{D}_{6}\right)$, no $\mathrm{H} / \mathrm{Ph}$ exchange between the $\mathrm{B}$ and $\mathrm{Si}$ atoms was observed.

\section{Characterization of compound 2d<smiles>COB(OC)OC</smiles>

${ }^{1} \mathrm{H}$ NMR $\left(395.9 \mathrm{MHz}, 24{ }^{\circ} \mathrm{C}, \mathrm{C}_{6} \mathrm{D}_{6}, \mathrm{ppm}\right): \delta=3.42$ (s, 9H, O-CH3 $) .{ }^{11} \mathrm{~B}$ NMR $(128.41 \mathrm{MHz}$, $\left.24{ }^{\circ} \mathrm{C}, \mathrm{C}_{6} \mathrm{D}_{6}, \mathrm{ppm}\right): \delta=17.8 .{ }^{11} \mathrm{~B}\left\{{ }^{1} \mathrm{H}\right\} \mathrm{NMR}\left(128.41 \mathrm{MHz}, 24{ }^{\circ} \mathrm{C}, \mathrm{C}_{6} \mathrm{D}_{6}, \mathrm{ppm}\right): \delta=17.8$.

\section{Characterization of compound $2 \mathrm{e}$}<smiles>COB1OB(OC)OB(OC)O1</smiles>

${ }^{1} \mathrm{H}$ NMR $\left(395.9 \mathrm{MHz}, 24{ }^{\circ} \mathrm{C}, \mathrm{C}_{6} \mathrm{D}_{6}, \mathrm{ppm}\right): \delta=3.34$ (s, 9H, O-CH$) .{ }^{11} \mathrm{~B}$ NMR $(128.41 \mathrm{MHz}$, $\left.24{ }^{\circ} \mathrm{C}, \mathrm{C}_{6} \mathrm{D}_{6}, \mathrm{ppm}\right): \delta=18.2 .{ }^{11} \mathrm{~B}\left\{{ }^{1} \mathrm{H}\right\} \mathrm{NMR}\left(128.41 \mathrm{MHz}, 24{ }^{\circ} \mathrm{C}, \mathrm{C}_{6} \mathrm{D}_{6}, \mathrm{ppm}\right): \delta=18.2$.

\section{Synthesis of compound $2 \mathrm{f}-2 \mathrm{~g}$}

Catalyst 1 (4.0 mg, $0.01 \mathrm{mmol})$, internal standard 1,3,5-tri-tert-butylbenzene (2.5 $\mathrm{mg}, 0.01$ mmol, 1 equiv.) and $0.5 \mathrm{~mL}$ of $\mathrm{C}_{6} \mathrm{D}_{6}$ were mixed in a J-Young NMR tube. The catecholborane, HBcat $(10.7 \mu \mathrm{L}, 0.10 \mathrm{mmol}, 10$ equiv.) was then added. The NMR tube was immersed in liquid nitrogen under vacuum to obtain a frozen solution. The J-Young NMR tube was lifted from liquid nitrogen and 1 bar of $\mathrm{CO}_{2}$ gas was then added. The reaction mixture was warmed to room temperature and then heated at $90{ }^{\circ} \mathrm{C}$. The reaction was monitored by NMR spectroscopy. The yields of products were reported according to the integration of ${ }^{1} \mathrm{H}$ NMR signals of catBOMe (2f) at $3.39 \mathrm{ppm}$ and $(\mathrm{catB})_{2} \mathrm{O}(\mathbf{2 g})$ at $6.72-6.75 \mathrm{ppm}$ with reference to the Me and $\mathrm{C}_{\mathrm{Ar}}-H$ protons $(1.35,7.42 \mathrm{ppm})$ of the internal standard, 1,3,5-tri-tert-butylbenzene. The chemical shifts of the products agree with the reported values in the literature and independent in-situ NMR-scale syntheses. ${ }^{\mathrm{S} 3}$-S4 The catalytic studies were repeated thrice. Full consumption of HBcat was observed after 24 hours.

\section{Characterization of compound $2 \mathrm{f}$}<smiles>COB1Oc2ccccc2O1</smiles>

${ }^{1} \mathrm{H}$ NMR $\left(395.9 \mathrm{MHz}, 24{ }^{\circ} \mathrm{C}, \mathrm{C}_{6} \mathrm{D}_{6}, \mathrm{ppm}\right): \delta=3.39$ (s, 3H, O-CH $), 6.72-6.75(\mathrm{~m}, 2 \mathrm{H}, \mathrm{Ar}-\mathrm{H}$, overlapped with 2g), $6.89-6.91$ (m, $2 \mathrm{H}, \mathrm{Ar}-\mathrm{H}$, overlapped with 2g). ${ }^{11} \mathrm{~B}$ NMR $(128.41 \mathrm{MHz}$, $\left.24{ }^{\circ} \mathrm{C}, \mathrm{C}_{6} \mathrm{D}_{6}, \mathrm{ppm}\right): \delta=21.3 .{ }^{11} \mathrm{~B}\left\{{ }^{1} \mathrm{H}\right\} \mathrm{NMR}\left(128.41 \mathrm{MHz}, 24{ }^{\circ} \mathrm{C}, \mathrm{C}_{6} \mathrm{D}_{6}, \mathrm{ppm}\right): \delta=21.3$. 
Characterization of compound $2 \mathrm{~g}$<smiles>c1ccc2c(c1)OB(B1Oc3ccccc3O1)O2</smiles>

${ }^{1} \mathrm{H}$ NMR (395.9 MHz, $\left.24{ }^{\circ} \mathrm{C}, \mathrm{C}_{6} \mathrm{D}_{6}, \mathrm{ppm}\right): \delta=6.72-6.75$ (m, 2H, Ar- $H$, overlapped with $2 \mathbf{f}$ ), $6.89-6.91$ (m, $2 \mathrm{H}, \mathrm{Ar}-H$, overlapped with $2 \mathrm{f}) .{ }^{11} \mathrm{~B} \mathrm{NMR}\left(128.41 \mathrm{MHz}, 24{ }^{\circ} \mathrm{C}, \mathrm{C}_{6} \mathrm{D}_{6}, \mathrm{ppm}\right): \delta$ $=22.2 .{ }^{11} \mathrm{~B}\left\{{ }^{1} \mathrm{H}\right\}$ NMR $\left(128.41 \mathrm{MHz}, 24{ }^{\circ} \mathrm{C}, \mathrm{C}_{6} \mathrm{D}_{6}, \mathrm{ppm}\right): \delta=22.2$.

General procedures for the catalytic hydroboration of carbon dioxide using 3 as the catalyst:

\section{Synthesis of compound $2 a-2 c$}

Catalyst 3 (1.3 mg, $0.0025 \mathrm{mmol})$, internal standard 1,3,5-trimethoxybenzene ( $8.4 \mathrm{mg}, 0.05$ mmol, 20 equiv.) and $0.5 \mathrm{~mL}$ of $\mathrm{C}_{6} \mathrm{D}_{6}$ were mixed in a J-Young NMR tube. Pinacolborane, HBpin (14.5 $\mu \mathrm{L}, 0.10 \mathrm{mmol}, 40$ equiv.) was then added. The NMR tube was immersed in liquid nitrogen under vacuum to obtain a frozen solution. The J-Young NMR tube was lifted from liquid nitrogen and 1 bar of $\mathrm{CO}_{2}$ gas was then added. The reaction mixture was warmed to room temperature and then heated at $90{ }^{\circ} \mathrm{C}$. The reaction was monitored by NMR spectroscopy. The yields of products were reported according to the integration of ${ }^{1} \mathrm{H}$ NMR signals of $\operatorname{pinBOC}(=\mathrm{O}) \mathrm{H}(\mathbf{2 a})$ at $0.93 \mathrm{ppm}$ and $(\operatorname{pinB})_{2} \mathrm{O}(\mathbf{2 c})$ at $1.02 \mathrm{ppm}$ with reference to the $-\mathrm{OMe}$ and $\mathrm{C}_{\mathrm{Ar}}-H$ protons $(3.36,6.20 \mathrm{ppm})$ of the internal standard, 1,3,5-trimethoxybenzene. The chemical shifts of the products agree with the reported values in the literature and independent in-situ NMR-scale syntheses. ${ }^{\text {S2 }}$ The catalytic studies were repeated thrice. Formation of $\mathbf{2} \mathbf{b}$ was not observed throughout the catalytic reaction. 
Table S1. Catalytic table for hydroboration of $\mathrm{CO}_{2}$ using $\mathrm{HBpin}$.

\begin{tabular}{|c|c|c|c|c|c|c|c|c|c|c|c|c|c|c|c|c|}
\hline \multirow{2}{*}{$\mathrm{S} / \mathrm{N}$} & \multirow{2}{*}{ Cat } & \multirow{2}{*}{$\mathrm{X}$} & \multirow{2}{*}{$\begin{array}{c}\mathrm{T} \\
\left({ }^{\circ} \mathrm{C}\right)\end{array}$} & \multirow{2}{*}{$\begin{array}{l}\text { Time } \\
\text { (h) }\end{array}$} & \multicolumn{4}{|c|}{ Yield } & \multicolumn{3}{|c|}{ TON } & \multicolumn{3}{|c|}{ TOF $\left(h^{-1}\right)$} & \multirow{2}{*}{ TON } & \multirow{2}{*}{ TOF $\left(h^{-1}\right)$} \\
\hline & & & & & $2 a$ & $2 b$ & $2 c$ & Total & $2 a$ & $2 b$ & $2 c$ & $2 a$ & $2 b$ & $2 c$ & & \\
\hline 1 & - & - & 110 & 13.00 & 0.00 & 0.00 & 0.10 & 0.10 & 0.00 & 0.00 & 0.00 & 0.00 & 0.00 & 0.00 & 0.00 & 0.00 \\
\hline 2 & $\mathrm{I}_{\mathrm{Me}}$ & 0.1 & 90 & 0.25 & 0.12 & 0.02 & 0.05 & 0.19 & 118.1 & 21.80 & 49.60 & 472.4 & 87.20 & 198.4 & 1.90 & 7.58 \\
\hline 4 & 1 & 10 & 24 & $\begin{array}{l}0.08 \\
0.17 \\
1.42\end{array}$ & $\begin{array}{l}0.41 \\
0.62 \\
0.92\end{array}$ & $\begin{array}{l}0.00 \\
0.00 \\
0.00\end{array}$ & $\begin{array}{l}0.02 \\
0.02 \\
0.02\end{array}$ & $\begin{array}{l}0.43 \\
0.64 \\
0.93\end{array}$ & $\begin{array}{l}4.12 \\
6.23 \\
9.15\end{array}$ & $\begin{array}{l}0.00 \\
0.00 \\
0.00\end{array}$ & $\begin{array}{l}0.17 \\
0.17 \\
0.20\end{array}$ & $\begin{array}{c}49.46 \\
37.38 \\
6.46\end{array}$ & $\begin{array}{l}0.00 \\
0.00 \\
0.00\end{array}$ & $\begin{array}{l}1.99 \\
1.02 \\
0.14\end{array}$ & $\begin{array}{l}4.29 \\
6.40 \\
9.35\end{array}$ & $\begin{array}{c}51.45 \\
38.40 \\
6.60\end{array}$ \\
\hline 5 & 1 & 10 & 60 & $\begin{array}{l}0.08 \\
0.17 \\
0.25 \\
0.33\end{array}$ & $\begin{array}{l}0.57 \\
0.80 \\
0.96 \\
0.96 \\
\end{array}$ & $\begin{array}{l}0.00 \\
0.00 \\
0.00 \\
0.00\end{array}$ & $\begin{array}{l}0.01 \\
0.01 \\
0.02 \\
0.04\end{array}$ & $\begin{array}{l}0.57 \\
0.81 \\
0.98 \\
0.99 \\
\end{array}$ & $\begin{array}{l}5.68 \\
7.96 \\
9.61 \\
9.62 \\
\end{array}$ & $\begin{array}{l}0.00 \\
0.00 \\
0.00 \\
0.00 \\
\end{array}$ & $\begin{array}{l}0.06 \\
0.13 \\
0.20 \\
0.38 \\
\end{array}$ & $\begin{array}{l}68.11 \\
47.78 \\
38.43 \\
28.86\end{array}$ & $\begin{array}{l}0.00 \\
0.00 \\
0.00 \\
0.00\end{array}$ & $\begin{array}{l}0.74 \\
0.75 \\
0.79 \\
1.14 \\
\end{array}$ & $\begin{array}{c}5.74 \\
8.09 \\
9.80 \\
10.00\end{array}$ & $\begin{array}{l}68.85 \\
48.53 \\
39.22 \\
30.00 \\
\end{array}$ \\
\hline $7^{a}$ & 1 & 10 & 90 & $\begin{array}{l}0.08 \\
0.17\end{array}$ & $\begin{array}{l}0.94 \\
0.98\end{array}$ & $\begin{array}{l}0.00 \\
0.00\end{array}$ & $\begin{array}{l}0.02 \\
0.02\end{array}$ & $\begin{array}{l}0.96 \\
0.99\end{array}$ & $\begin{array}{l}9.43 \\
9.79\end{array}$ & $\begin{array}{l}0.00 \\
0.00\end{array}$ & $\begin{array}{l}0.15 \\
0.21\end{array}$ & $\begin{array}{c}113.21 \\
58.73\end{array}$ & $\begin{array}{l}0.00 \\
0.00\end{array}$ & $\begin{array}{l}1.85 \\
1.27\end{array}$ & $\begin{array}{c}9.59 \\
10.00\end{array}$ & $\begin{array}{c}115.06 \\
60.00\end{array}$ \\
\hline$\overline{88}$ & $\overline{3}$ & 2.5 & 90 & $\begin{array}{l}0.08 \\
0.17\end{array}$ & $\begin{array}{l}0.85 \\
0.94\end{array}$ & $\begin{array}{l}0.00 \\
0.00\end{array}$ & $\begin{array}{l}0.06 \\
0.06\end{array}$ & $\begin{array}{l}0.91 \\
0.99\end{array}$ & $\begin{array}{l}34.17 \\
37.77\end{array}$ & $\begin{array}{l}0.00 \\
0.00\end{array}$ & $\begin{array}{l}2.20 \\
2.23\end{array}$ & $\begin{array}{l}427.08 \\
226.62\end{array}$ & $\begin{array}{l}0.00 \\
0.00\end{array}$ & $\begin{array}{l}27.54 \\
13.38\end{array}$ & $\begin{array}{l}36.37 \\
40.00\end{array}$ & $\begin{array}{l}454.62 \\
240.00\end{array}$ \\
\hline
\end{tabular}

Conditions: $0.10 \mathrm{mmol}$ of HBpin in $\mathrm{C}_{6} \mathrm{D}_{6}(\sim 0.5 \mathrm{ml})$, amount of catalyst is calculated and then added to obtain the desired catalytic loading, 1 bar 
of $\mathrm{CO}_{2}$. The yield was obtained by ${ }^{1} \mathrm{H}$ NMR using the $\mathrm{Ar}-\mathrm{H}$ and OMe protons of 1,3,5-trimethoxybenzene as an internal standard. TON and

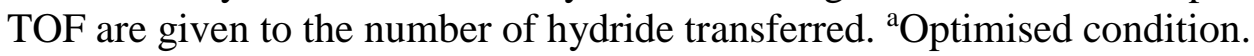

Table S2. Catalytic table for hydroboration of $\mathrm{CO}_{2}$ using $\mathrm{HB}$ pin in $\mathrm{C}_{6} \mathrm{D}_{6}$ containing $\mathrm{O}_{2}$ and/or $\mathrm{H}_{2} \mathrm{O}$.

\begin{tabular}{|c|c|c|c|c|c|c|c|c|c|c|c|c|c|c|}
\hline & & & $\mathcal{O}_{\mathrm{O}^{\prime}}^{\mathrm{B}^{\prime}}$ & $\begin{array}{l}\text { Cat } \\
\text { Tem }\end{array}$ & $\begin{array}{l}\text { yst } 1 \text { ( } \\
\mathrm{C}_{6} \mathrm{D}_{6} \\
\text { rature } \\
\text { Time }\end{array}$ & $\stackrel{\left.{ }^{\circ} \mathrm{C}\right),}{\longrightarrow}$ & $2 a$ & & $2 b$ & + & $2 c$ & & & \\
\hline \multirow{2}{*}{$\mathrm{S} / \mathrm{N}$} & \multirow{2}{*}{ Condition } & \multirow{2}{*}{$\begin{array}{c}\text { Time } \\
\text { (h) }\end{array}$} & \multicolumn{4}{|c|}{ Yield } & \multicolumn{3}{|c|}{ TON } & \multicolumn{3}{|c|}{ TOF $\left(h^{-1}\right)$} & \multirow{2}{*}{ TON } & \multirow{2}{*}{ TOF $\left(h^{-1}\right)$} \\
\hline & & & $2 \mathbf{a}$ & $2 \mathbf{b}$ & $2 c$ & Total & $2 \mathbf{a}$ & $2 b$ & $2 c$ & $2 a$ & $2 \mathbf{b}$ & $2 c$ & & \\
\hline 1 & $78 \mathrm{ppm} \mathrm{H}_{2} \mathrm{O}^{\mathrm{a}}$ & 0.33 & 0.87 & 0.00 & 0.12 & 0.98 & 8.60 & 0.00 & 1.21 & 25.80 & 0.00 & 3.64 & 9.81 & 29.44 \\
\hline 2 & Presence of air ${ }^{b}$ & 0.33 & 0.77 & 0.00 & 0.09 & 0.85 & 7.65 & 0.00 & 0.88 & 22.96 & 0.00 & 2.64 & 8.54 & 25.61 \\
\hline
\end{tabular}

Conditions: $0.10 \mathrm{mmol}$ of HBpin in $\mathrm{C}_{6} \mathrm{D}_{6}(\sim 0.5 \mathrm{ml})$, amount of catalyst is calculated and then added to obtain the desired catalytic loading, 1 bar of $\mathrm{CO}_{2}$. The yield was obtained by ${ }^{1} \mathrm{H}$ NMR using the $\mathrm{Ar}-\mathrm{H}$ and OMe protons of 1,3,5-trimethoxybenzene as an internal standard. TON and TOF are given to the number of hydride transferred. ${ }^{a} \mathrm{~A} 100 \mathrm{~mL}$ packaging of $\mathrm{C}_{6} \mathrm{D}_{6}(\mathrm{D}, 99.5 \%$, containing 78 ppm residual water) was purchased from Cambridge Isotope Laboratories, Inc and subjected to freeze-pump-thaw cycle to remove air. ${ }^{\mathrm{b}}$ Activated molecular sieve was added into a $100 \mathrm{~mL}$ packaging of $\mathrm{C}_{6} \mathrm{D}_{6}\left(\mathrm{D}, 99.5 \%\right.$, containing $78 \mathrm{ppm}$ residual water) and left to stand for 5 days, occasionally agitating the solvent. $\mathrm{C}_{6} \mathrm{D}_{6} \mathrm{was} \mathrm{w}_{\mathrm{i}}$ filtered under air for catalysis. ${ }^{c} \mathrm{~A} 100 \mathrm{~mL}$ packaging of $\mathrm{C}_{6} \mathrm{D}_{6}(\mathrm{D}, 99.5 \%$, containing $78 \mathrm{ppm}$ residual water) was purchased and used directly without further drying and purification. 
Table S3. Catalytic table for hydroboration of $\mathrm{CO}_{2}$ using $\mathrm{BH}_{3} \cdot \mathrm{SMe}_{2}$.

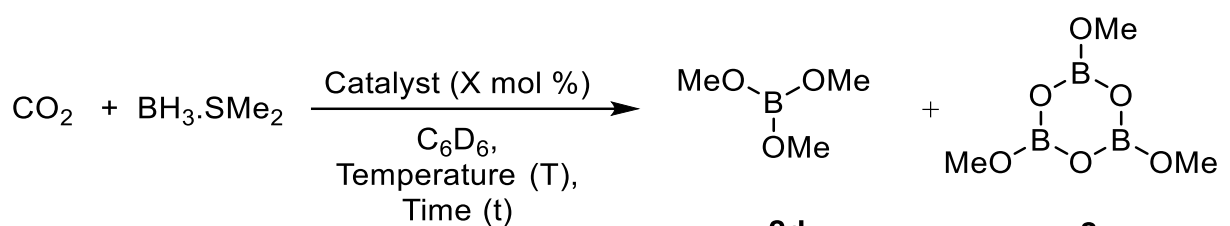

2d

\begin{tabular}{|c|c|c|c|c|c|c|c|c|c|c|c|c|c|}
\hline \multirow{2}{*}{$\mathrm{S} / \mathrm{N}$} & \multirow{2}{*}{ Cat } & \multirow{2}{*}{$\mathrm{X}$} & \multirow{2}{*}{$\mathrm{T}\left({ }^{\mathrm{o}} \mathrm{C}\right)$} & \multirow{2}{*}{$\mathrm{t}(\mathrm{h})$} & \multicolumn{3}{|c|}{ Yield } & \multicolumn{2}{|c|}{ TON } & \multicolumn{2}{|c|}{ TOF $\left(h^{-1}\right)$} & \multirow{2}{*}{ TON } & \multirow{2}{*}{$\operatorname{TOF}\left(\mathrm{h}^{-1}\right)$} \\
\hline & & & & & $2 d$ & $2 e$ & Total & $2 d$ & $2 e$ & $2 d$ & $2 \mathrm{e}$ & & \\
\hline 1 & - & - & 90 & 24 & 0.00 & 0.00 & 0.00 & - & - & - & - & - & - \\
\hline 2 & $\mathrm{I}_{\mathrm{Me}}$ & 0.1 & 90 & 0.50 & 0.00 & 0.09 & 0.09 & 0.00 & 0.13 & 0 & 2.66 & 1.33 & 2.66 \\
\hline \multirow[t]{2}{*}{3} & \multirow[t]{2}{*}{1} & \multirow[t]{2}{*}{10} & \multirow[t]{2}{*}{60} & 0.33 & 0.02 & 0.21 & 0.24 & 0.23 & 2.15 & 0.68 & 6.44 & 2.37 & 7.12 \\
\hline & & & & 1.33 & 0.15 & 0.83 & 0.99 & 1.51 & 8.34 & 1.14 & 6.25 & 9.85 & 7.39 \\
\hline \multirow[t]{2}{*}{$4^{\mathrm{a}}$} & \multirow[t]{2}{*}{1} & \multirow[t]{2}{*}{10} & \multirow[t]{2}{*}{90} & 0.08 & 0.02 & 0.14 & 0.16 & 0.17 & 1.43 & 2.05 & 17.21 & 1.60 & 19.26 \\
\hline & & & & 0.50 & 0.18 & 0.81 & 0.99 & 1.77 & 8.13 & 3.53 & 16.27 & 9.90 & 19.80 \\
\hline
\end{tabular}

$2 e$

Conditions: $0.10 \mathrm{mmol} \mathrm{BH}_{3} . \mathrm{SMe}_{2}$ in $\mathrm{C}_{6} \mathrm{D}_{6}(\sim 0.5 \mathrm{ml})$, amount of catalyst is calculated and then added to obtain the desired catalytic loading, 1 bar of $\mathrm{CO}_{2}$. The yield was obtained by ${ }^{1} \mathrm{H}$ NMR using the $-\mathrm{Me}$ and $-\mathrm{Ph}$ protons of methyltriphenylsilane as an internal standard. TON and TOF are given to the number of hydride transferred. ${ }^{a}$ Optimised condition. There was no reaction between $\mathrm{BH}_{3} . \mathrm{SMe}_{2}$ and methyltriphenylsilane 
Table S4. Catalytic table for hydroboration of $\mathrm{CO}_{2}$ using HBcat.

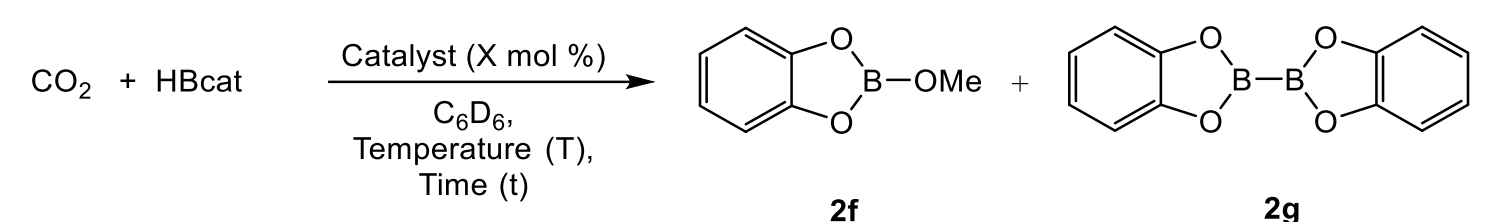
Time (t)

$2 f$

$\mathbf{2 g}$

\begin{tabular}{|c|c|c|c|c|c|c|c|c|c|c|c|c|c|}
\hline \multirow{2}{*}{$\mathrm{S} / \mathrm{N}$} & \multirow{2}{*}{ Cat } & \multirow{2}{*}{$X$} & \multirow{2}{*}{$\mathrm{T}\left({ }^{\circ} \mathrm{C}\right)$} & \multirow{2}{*}{$\mathrm{t}(\mathrm{h})$} & \multicolumn{3}{|c|}{ Yield } & \multicolumn{2}{|c|}{ TON } & \multicolumn{2}{|c|}{ TOF $\left(h^{-1}\right)$} & \multirow{2}{*}{ TON } & \multirow{2}{*}{$\operatorname{TOF}\left(\mathrm{h}^{-1}\right)$} \\
\hline & & & & & $2 f$ & $2 \mathrm{~g}$ & Total & $2 f$ & $2 \mathrm{~g}$ & $2 f$ & $2 \mathrm{~g}$ & & \\
\hline 1 & - & - & 90 & 24 & 0.00 & 0.00 & 0.00 & - & - & - & - & - & - \\
\hline 2 & 1 & 1 & 90 & 24 & 0.01 & 0.40 & 0.41 & 0.77 & 40.11 & 0.03 & 1.67 & 4.09 & 0.17 \\
\hline 3 & 1 & 5 & 90 & 24 & 0.01 & 0.48 & 0.49 & 0.20 & 7.70 & 0.08 & 0.32 & 4.85 & 0.20 \\
\hline $4^{a}$ & 1 & 10 & 90 & 24 & 0.12 & 0.70 & 0.82 & 1.2 & 7.00 & 0.05 & 0.29 & 8.20 & 0.34 \\
\hline
\end{tabular}

Conditions: $0.10 \mathrm{mmol}$ of HBcat in $\mathrm{C}_{6} \mathrm{D}_{6}(\sim 0.5 \mathrm{ml})$, amount of catalyst is calculated and then added to obtain the desired catalytic loading, 1 bar of $\mathrm{CO}_{2}$. The yield was obtained by ${ }^{1} \mathrm{H}$ NMR using the Ar and $t \mathrm{Bu}$ protons of 1,3,5-tri-tert-butylbenzene as an internal standard. TON and TOF are given to the number of hydride transferred. ${ }^{\mathrm{a}}$ Optimised condition. 


\section{General procedures for the catalytic hydroboration of aldehyde, ketone and pyridine derivatives using 1 as the catalyst:}

Catalyst 1 (4.0 mg, $0.01 \mathrm{mmol}$ ) and $0.5 \mathrm{~mL}$ of $\mathrm{C}_{6} \mathrm{D}_{6}$ were added into a J-Young NMR tube. Pinacolborane, HBpin $(16.0 \mu \mathrm{L}, 0.11 \mathrm{mmol}, 10.1$ equiv. $)$ and substrates $(0.10 \mathrm{mmol}, 10$ equiv. $)$ were then added. The reaction conditions are indicated in Table S5 - S7 and the reactions were followed by NMR spectroscopy to determine their yields. The chemical shifts of the products are in good agreement with the reported values in the literatures. All the catalytic trials were repeated in triplicate.

\section{$\underline{\text { Isolation of product 5a-t and 7a-j }}$}

Upon completion of the catalyses, the resulting crude solutions were filtered and the filtrates were dried under vacuum. The dried residues were then further extracted with hexane. The resultant borate esters were isolated by removing volatiles under vacuum for a few hours.

\section{$\underline{\text { Isolation of product 9a-f }}$}

Upon completion of the catalyses, the resulting crude solutions were filtered and the filtrates were dried under vacuum. The dried residues were then further extracted with either diethyl ether, pentane or hexane. The resultant N-boryl-1,4-dihydropyridine derivatives were isolated by removing volatiles under vacuum for a few hours.

\section{Characterization of compound 5a:}<smiles>CCCCOCc1ccccc1</smiles>

${ }^{1} \mathrm{H}$ NMR (395.9 MHz, $\left.24{ }^{\circ} \mathrm{C}, \mathrm{C}_{6} \mathrm{D}_{6}, \mathrm{ppm}\right): \delta=7.30\left(\mathrm{~d}, 2 \mathrm{H}, \mathrm{Ar}-H,{ }^{3} J_{\mathrm{H}, \mathrm{H}}=7.2 \mathrm{~Hz}\right), 7.13(\mathrm{t}$, $\left.2 \mathrm{H}, \operatorname{Ar}-H,{ }^{3} J_{\mathrm{H}, \mathrm{H}}=7.5 \mathrm{~Hz}\right), 7.07-7.03(\mathrm{~m}, 1 \mathrm{H}, \mathrm{Ar}-\mathrm{H}), 4.94\left(\mathrm{~s}, 2 \mathrm{H}, \mathrm{O}-\mathrm{CH}_{2}\right), 1.04(\mathrm{~s}, 12 \mathrm{H}$, Bpin- $\left.\mathrm{CH}_{3}\right) .{ }^{11} \mathrm{~B}\left\{{ }^{1} \mathrm{H}\right\}$ NMR $\left(128.41 \mathrm{MHz}, 24{ }^{\circ} \mathrm{C}, \mathrm{C}_{6} \mathrm{D}_{6}, \mathrm{ppm}\right): \delta=21.8$.

\section{Characterization of compound $5 \mathrm{~b}$ :}<smiles>Cc1ccc(CO[R16](c2ccccc2)c2ccc(C)cc2)cc1</smiles>

${ }^{1} \mathrm{H}$ NMR $\left(395.9 \mathrm{MHz}, 24{ }^{\circ} \mathrm{C}, \mathrm{C}_{6} \mathrm{D}_{6}, \mathrm{ppm}\right): \delta=7.25\left(\mathrm{~d}, 2 \mathrm{H}, \mathrm{Ar}-H,{ }^{3} J_{\mathrm{H}, \mathrm{H}}=7.7 \mathrm{~Hz}\right), 6.96(\mathrm{~d}$, $\left.2 \mathrm{H}, \mathrm{Ar}-\mathrm{H},{ }^{3} J_{\mathrm{H}, \mathrm{H}}=8.2 \mathrm{~Hz}\right), 4.95\left(\mathrm{~s}, 2 \mathrm{H}, \mathrm{O}-\mathrm{CH}_{2}\right), 2.08\left(\mathrm{~s}, 3 \mathrm{H},-\mathrm{CH}_{3}\right), 1.05\left(\mathrm{~s}, 12 \mathrm{H}, \mathrm{Bpin}-\mathrm{CH}_{3}\right)$. ${ }^{11} \mathrm{~B}\left\{{ }^{1} \mathrm{H}\right\}$ NMR $\left(128.41 \mathrm{MHz}, 24{ }^{\circ} \mathrm{C}, \mathrm{C}_{6} \mathrm{D}_{6}, \mathrm{ppm}\right): \delta=21.9$.

\section{Characterization of compound $5 \mathrm{c}$ :}<smiles>COc1cccc(CO[R16](=O)c2ccccc2)c1</smiles>

${ }^{1} \mathrm{H}$ NMR $\left(395.9 \mathrm{MHz}, 24{ }^{\circ} \mathrm{C}, \mathrm{C}_{6} \mathrm{D}_{6}, \mathrm{ppm}\right): \delta=7.09-7.06(\mathrm{~m}, 1 \mathrm{H}, \mathrm{Ar}-H), 6.99$ (s, $\left.1 \mathrm{H}, \mathrm{Ar}-H\right)$, $6.92\left(\mathrm{~d}, 1 \mathrm{H}, \mathrm{Ar}-H,{ }^{3} J_{\mathrm{H}, \mathrm{H}}=7.2 \mathrm{~Hz}\right), 6.71\left(\mathrm{dd}, 1 \mathrm{H}, \mathrm{Ar}-H,{ }^{3} J_{\mathrm{H}, \mathrm{H}}=2.3,8.2 \mathrm{~Hz}\right), 4.94(\mathrm{~s}, 2 \mathrm{H}, \mathrm{O}-$ $\left.\mathrm{CH}_{2}\right), 3.32$ (s, 3H, O-CH $), 1.05$ (s, $\left.12 \mathrm{H}, \mathrm{Bpin}-\mathrm{CH}_{3}\right) .{ }^{11} \mathrm{~B}\left\{{ }^{1} \mathrm{H}\right\} \mathrm{NMR}\left(128.41 \mathrm{MHz}, 24{ }^{\circ} \mathrm{C}\right.$, $\left.\mathrm{C}_{6} \mathrm{D}_{6}, \mathrm{ppm}\right): \delta=21.8$. 


\section{Characterization of compound 5d:}<smiles>CCCCOCCC1CCCCC1</smiles>

${ }^{1} \mathrm{H}$ NMR (395.9 MHz, $\left.24{ }^{\circ} \mathrm{C}, \mathrm{C}_{6} \mathrm{D}_{6}, \mathrm{ppm}\right): \delta=3.73\left(\mathrm{~d}, 2 \mathrm{H}, \mathrm{O}-\mathrm{CH}_{2},{ }^{3} \mathrm{~J}_{\mathrm{H}, \mathrm{H}}=4.5 \mathrm{~Hz}\right), 1.71-$ $1.38(\mathrm{~m}, 11 \mathrm{H}, \mathrm{Cy}-H), 1.07\left(\mathrm{~s}, 12 \mathrm{H}, \mathrm{Bpin}-\mathrm{CH}_{3}\right) .{ }^{11} \mathrm{~B}\left\{{ }^{1} \mathrm{H}\right\} \mathrm{NMR}\left(128.41 \mathrm{MHz}, 24{ }^{\circ} \mathrm{C}, \mathrm{C}_{6} \mathrm{D}_{6}\right.$, ppm): $\delta=21.6$.

\section{Characterization of compound 5e:}<smiles>COC(=O)c1ccc(COc2ccccc2)cc1</smiles>

${ }^{1} \mathrm{H}$ NMR (395.9 MHz, $\left.24{ }^{\circ} \mathrm{C}, \mathrm{C}_{6} \mathrm{D}_{6}, \mathrm{ppm}\right): \delta=8.08\left(\mathrm{~d}, 2 \mathrm{H}, \mathrm{Ar}-H,{ }^{3} J_{\mathrm{H}, \mathrm{H}}=8.2 \mathrm{~Hz}\right), 7.22(\mathrm{~d}$, $\left.2 \mathrm{H}, \mathrm{Ar}-\mathrm{H},{ }^{3} \mathrm{~J}_{\mathrm{H}, \mathrm{H}}=7.7 \mathrm{~Hz}\right), 4.85\left(\mathrm{~s}, 2 \mathrm{H}, \mathrm{O}-\mathrm{CH}_{2}\right), 3.47$ (s, 3H, O-CH $), 1.03$ (s, 12H, Bpin$\left.\mathrm{CH}_{3}\right) .{ }^{11} \mathrm{~B}\left\{{ }^{1} \mathrm{H}\right\}$ NMR $\left(128.41 \mathrm{MHz}, 24{ }^{\circ} \mathrm{C}, \mathrm{C}_{6} \mathrm{D}_{6}, \mathrm{ppm}\right): \delta=21.8$.

\section{Characterization of compound $5 f$ :}<smiles>c1ccc([PbH2]OCc2cccs2)cc1</smiles>

${ }^{1} \mathrm{H}$ NMR (395.9 MHz, $\left.24{ }^{\circ} \mathrm{C}, \mathrm{C}_{6} \mathrm{D}_{6}, \mathrm{ppm}\right): \delta=6.88\left(\mathrm{~d}, 1 \mathrm{H}, \mathrm{Ar}-H,{ }^{3} J_{\mathrm{H}, \mathrm{H}}=5.0 \mathrm{~Hz}\right), 6.82(\mathrm{~d}$, $\left.1 \mathrm{H}, \mathrm{Ar}-H,{ }^{3} J_{\mathrm{H}, \mathrm{H}}=3.2 \mathrm{~Hz}\right), 6.69-6.67(\mathrm{~m}, 1 \mathrm{H}, \mathrm{Ar}-H), 4.98\left(\mathrm{~s}, 2 \mathrm{H}, \mathrm{O}-\mathrm{CH}_{2}\right), 1.04(\mathrm{~s}, 12 \mathrm{H}$, Bpin- $\left.\mathrm{CH}_{3}\right) .{ }^{11} \mathrm{~B}\left\{{ }^{1} \mathrm{H}\right\}$ NMR $\left(128.41 \mathrm{MHz}, 24{ }^{\circ} \mathrm{C}, \mathrm{C}_{6} \mathrm{D}_{6}, \mathrm{ppm}\right): \delta=21.8$.

\section{Characterization of compound 5g:}

Cp1

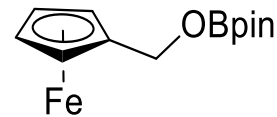

Cp2

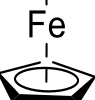

${ }^{1} \mathrm{H}$ NMR (395.9 MHz, $\left.24{ }^{\circ} \mathrm{C}, \mathrm{C}_{6} \mathrm{D}_{6}, \mathrm{ppm}\right): \delta=4.76\left(\mathrm{~s}, 2 \mathrm{H}, \mathrm{O}-\mathrm{CH}_{2}\right), 4.21\left(\mathrm{t}, 2 \mathrm{H}, \mathrm{Cp} 1-H,{ }^{3} J_{\mathrm{H}, \mathrm{H}}\right.$ $=1.8 \mathrm{~Hz}), 3.98(\mathrm{~s}, 5 \mathrm{H}, \mathrm{Cp} 2-H), 3.95\left(\mathrm{t}, 2 \mathrm{H}, \mathrm{Cp} 1-H,{ }^{3} J_{\mathrm{H}, \mathrm{H}}=1.8 \mathrm{~Hz}\right), 1.08$ (s, 12H, Bpin$\left.\mathrm{CH}_{3}\right) .{ }^{11} \mathrm{~B}\left\{{ }^{1} \mathrm{H}\right\} \mathrm{NMR}\left(128.41 \mathrm{MHz}, 24{ }^{\circ} \mathrm{C}, \mathrm{C}_{6} \mathrm{D}_{6}, \mathrm{ppm}\right): \delta=21.8$.

\section{Characterization of compound 5h:}<smiles>CCCCOCCC1CC=CCC1</smiles>

${ }^{1} \mathrm{H}$ NMR $\left(395.9 \mathrm{MHz}, 24{ }^{\circ} \mathrm{C}, \mathrm{C}_{6} \mathrm{D}_{6}, \mathrm{ppm}\right): \delta=5.59(\mathrm{~s}, 2 \mathrm{H}, \mathrm{C}=\mathrm{CH}), 3.78\left(\mathrm{~d}, 2 \mathrm{H}, \mathrm{O}-\mathrm{CH}_{2},{ }^{3} J_{\mathrm{H}, \mathrm{H}}\right.$ $=5.9 \mathrm{~Hz}), 2.03-1.67(\mathrm{~m}, 7 \mathrm{H}, \mathrm{Cy}-H), 1.06\left(\mathrm{~s}, 12 \mathrm{H}, \mathrm{Bpin}-\mathrm{CH}_{3}\right) .{ }^{11} \mathrm{~B}\left\{{ }^{1} \mathrm{H}\right\} \mathrm{NMR}(128.41 \mathrm{MHz}$, $\left.24{ }^{\circ} \mathrm{C}, \mathrm{C}_{6} \mathrm{D}_{6}, \mathrm{ppm}\right): \delta=21.6$.

\section{Characterization of compound $5 \mathbf{j}$ :}<smiles>COC(=O)c1ccc(/C=C/COc2ccccc2)cc1OC</smiles>

${ }^{1} \mathrm{H}$ NMR $\left(395.9 \mathrm{MHz}, 24{ }^{\circ} \mathrm{C}, \mathrm{C}_{6} \mathrm{D}_{6}, \mathrm{ppm}\right): \delta=6.90\left(\mathrm{~d}, 1 \mathrm{H}, \mathrm{Ar}-H,{ }^{3} J_{\mathrm{H}, \mathrm{H}}=8.2\right), 6.75(\mathrm{dd}, 1 \mathrm{H}$, Ar- $\left.H,{ }^{3} J_{\mathrm{H}, \mathrm{H}}=1.8,8.2 \mathrm{~Hz}\right), 6.68\left(\mathrm{~d}, 1 \mathrm{H}, \mathrm{Ar}-H,{ }^{3} J_{\mathrm{H}, \mathrm{H}}=1.3 \mathrm{~Hz}\right), 6.59\left(\mathrm{~d}, 1 \mathrm{H}, \mathrm{Ar}-\mathrm{CH},{ }^{3} J_{\mathrm{H}, \mathrm{H}}=\right.$ $15.9 \mathrm{~Hz}), 6.12\left(\mathrm{dt}, 1 \mathrm{H}, \mathrm{Ar}-\mathrm{C}=\mathrm{CH},{ }^{3} J_{\mathrm{H}, \mathrm{H}}=5.2,15.9 \mathrm{~Hz}\right), 4.58\left(\mathrm{dd}, 2 \mathrm{H}, \mathrm{O}-\mathrm{CH}_{2},{ }^{3} J_{\mathrm{H}, \mathrm{H}}=1.6,5.2\right.$ $\mathrm{Hz}), 3.26\left(\mathrm{~s}, 3 \mathrm{H}, \mathrm{CO}_{2}-\mathrm{CH}_{3}\right), 1.90\left(\mathrm{~s}, 3 \mathrm{H}, \mathrm{ArO}-\mathrm{CH}_{3}\right), 1.08\left(\mathrm{~s}, 12 \mathrm{H}, \mathrm{Bpin}-\mathrm{CH}_{3}\right) .{ }^{13} \mathrm{C}\left\{{ }^{1} \mathrm{H}\right\} \mathrm{NMR}$ 
(99.5 MHz, $\left.24{ }^{\circ} \mathrm{C}, \mathrm{C}_{6} \mathrm{D}_{6}, \mathrm{ppm}\right): \delta=168.1(C=\mathrm{O}), 151.9(\mathrm{Ar}-C), 140.2(\mathrm{Ar}-C), 136.1$

$(\mathrm{CH}=\mathrm{CH}), 130.3(\mathrm{Ar}-\mathrm{C}), 127.7(\mathrm{Ar}-\mathrm{C}), 123.3(\mathrm{CH}=\mathrm{CH}), 119.2(\mathrm{Ar}-\mathrm{C}), 110.6(\mathrm{Ar}-\mathrm{C}), 82.8$

$\left(C\left(\mathrm{CH}_{3}\right)_{2}-\mathrm{Bpin}\right), 65.5\left(\mathrm{O}-\mathrm{CH}_{2}\right), 55.3\left(\mathrm{CO}_{2}-\mathrm{CH}_{3}\right), 24.7\left(\mathrm{Bpin}-\mathrm{CH}_{3}\right), 20.3\left(\mathrm{O}-\mathrm{CH}_{3}\right)$.

\section{Characterization of compound 5k:}<smiles>CC(C)(C)CO[18OH]</smiles>

${ }^{1} \mathrm{H}$ NMR (395.9 MHz, $\left.24{ }^{\circ} \mathrm{C}, \mathrm{C}_{6} \mathrm{D}_{6}, \mathrm{ppm}\right): \delta=3.65$ (s, 2H, O-CH2), 1.07 (s, 12H, Bpin- $\mathrm{CH}_{3}$ ), $0.90\left(\mathrm{~s}, 9 \mathrm{H},-\mathrm{CH}_{3}\right) .{ }^{11} \mathrm{~B}\left\{{ }^{1} \mathrm{H}\right\} \mathrm{NMR}\left(128.41 \mathrm{MHz}, 24{ }^{\circ} \mathrm{C}, \mathrm{C}_{6} \mathrm{D}_{6}, \mathrm{ppm}\right): \delta=21.7$.

\section{Characterization of compound 5l:}

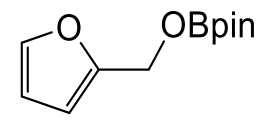

${ }^{1} \mathrm{H}$ NMR $\left(395.9 \mathrm{MHz}, 24{ }^{\circ} \mathrm{C}, \mathrm{C}_{6} \mathrm{D}_{6}, \mathrm{ppm}\right): \delta=7.05(\mathrm{~m}, 1 \mathrm{H}, \mathrm{Ar}-H), 6.13\left(\mathrm{~d}, 1 \mathrm{H}, \mathrm{Ar}-H,{ }^{3} J_{\mathrm{H}, \mathrm{H}}=\right.$ $3.2 \mathrm{~Hz}), 6.02-6.01(\mathrm{~m}, 1 \mathrm{H}, \mathrm{Ar}-\mathrm{H}), 4.85$ (s, 2H, O-CH$), 1.03\left(\mathrm{~s}, 12 \mathrm{H}, \mathrm{Bpin}-\mathrm{CH}_{3}\right) .{ }^{11} \mathrm{~B}\left\{{ }^{1} \mathrm{H}\right\}$ NMR (128.41 MHz, $\left.24{ }^{\circ} \mathrm{C}, \mathrm{C}_{6} \mathrm{D}_{6}, \mathrm{ppm}\right): \delta=21.8$.

\section{Characterization of compound $5 \mathrm{~m}$ :}

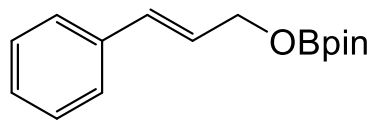

${ }^{1} \mathrm{H}$ NMR $\left(395.9 \mathrm{MHz}, 24{ }^{\circ} \mathrm{C}, \mathrm{C}_{6} \mathrm{D}_{6}, \mathrm{ppm}\right): \delta=7.18\left(\mathrm{~d}, 2 \mathrm{H}, \mathrm{Ar}-H,{ }^{3} J_{\mathrm{H}, \mathrm{H}}=7.2 \mathrm{~Hz}\right), 7.08(\mathrm{t}, 2 \mathrm{H}$, $\left.\operatorname{Ar}-H,{ }^{3} J_{\mathrm{H}, \mathrm{H}}=7.2 \mathrm{~Hz}\right), 7.03-6.99(\mathrm{~m}, 1 \mathrm{H}, \mathrm{Ar}-H), 6.61\left(\mathrm{~d}, 1 \mathrm{H}, \mathrm{Ar}-\mathrm{C} H,{ }^{3} J_{\mathrm{H}, \mathrm{H}}=16.3 \mathrm{~Hz}\right), 6.17$ $\left(\mathrm{dt}, 1 \mathrm{H}, \mathrm{Ar}-\mathrm{C}=\mathrm{CH},{ }^{3} J_{\mathrm{H}, \mathrm{H}}=5.2,15.9 \mathrm{~Hz}\right), 4.54\left(\mathrm{dd}, 2 \mathrm{H}, \mathrm{O}-\mathrm{CH}_{2},{ }^{3} J_{\mathrm{H}, \mathrm{H}}=5.2 \mathrm{~Hz},{ }^{4} J_{\mathrm{H}, \mathrm{H}}=1.6\right.$ $\mathrm{Hz}), 1.06$ (s, 12H, Bpin- $\left.\mathrm{CH}_{3}\right) .{ }^{11} \mathrm{~B}\left\{{ }^{1} \mathrm{H}\right\}$ NMR (128.41 MHz, $\left.24{ }^{\circ} \mathrm{C}, \mathrm{C}_{6} \mathrm{D}_{6}, \mathrm{ppm}\right): \delta=21.8$.

\section{Characterization of compound 5n:}<smiles></smiles>

${ }^{1} \mathrm{H}$ NMR (395.9 MHz, $\left.24{ }^{\circ} \mathrm{C}, \mathrm{C}_{6} \mathrm{D}_{6}, \mathrm{ppm}\right): \delta=7.09-7.05(\mathrm{~m}, 2 \mathrm{H}, \mathrm{Ar}-H), 6.78-6.74(\mathrm{~m}, 2 \mathrm{H}$, $\mathrm{Ar}-\mathrm{H}), 4.80$ (s, 2H, O-CH$), 1.04$ (s, $\left.12 \mathrm{H}, \mathrm{Bpin}-\mathrm{CH}_{3}\right) .{ }^{11} \mathrm{~B}\left\{{ }^{1} \mathrm{H}\right\} \mathrm{NMR}\left(128.41 \mathrm{MHz}, 24{ }^{\circ} \mathrm{C}\right.$, $\left.\mathrm{C}_{6} \mathrm{D}_{6}, \mathrm{ppm}\right): \delta=21.8$.

\section{Characterization of compound 5p:}<smiles>Brc1ccc(CO[SeH2]c2ccccc2)cc1</smiles>

${ }^{1} \mathrm{H}$ NMR $\left(395.9 \mathrm{MHz}, 24{ }^{\circ} \mathrm{C}, \mathrm{C}_{6} \mathrm{D}_{6}, \mathrm{ppm}\right): \delta=7.21\left(\mathrm{~d}, 2 \mathrm{H}, \mathrm{Ar}-H,{ }^{3} J_{\mathrm{H}, \mathrm{H}}=8.6 \mathrm{~Hz}\right), 6.93(\mathrm{~d}$, $2 \mathrm{H}, \mathrm{Ar}-\mathrm{H},{ }^{3} \mathrm{~J}_{\mathrm{H}, \mathrm{H}}=8.6 \mathrm{~Hz}$ ), $4.73\left(\mathrm{~s}, 2 \mathrm{H}, \mathrm{O}-\mathrm{CH}_{2}\right), 1.03\left(\mathrm{~s}, 12 \mathrm{H}, \mathrm{Bpin}-\mathrm{CH}_{3}\right) .{ }^{11} \mathrm{~B}\left\{{ }^{1} \mathrm{H}\right\} \mathrm{NMR}$ (128.41 MHz, $\left.24{ }^{\circ} \mathrm{C}, \mathrm{C}_{6} \mathrm{D}_{6}, \mathrm{ppm}\right): \delta=21.8$.

\section{Characterization of compound 5q:}<smiles>CC(=Cc1ccccc1)COC(C)(C)C</smiles>

${ }^{1} \mathrm{H}$ NMR $\left(395.9 \mathrm{MHz}, 24{ }^{\circ} \mathrm{C}, \mathrm{C}_{6} \mathrm{D}_{6}, \mathrm{ppm}\right): \delta=7.20-7.12$ (m, $4 \mathrm{H}$ [overlap with $\mathrm{C}_{6} \mathrm{D}_{6}$ ], Ar$H), 7.06-7.02(\mathrm{~m}, 1 \mathrm{H}, \mathrm{Ar}-H), 6.69(\mathrm{~s}, 1 \mathrm{H}, \mathrm{C}=\mathrm{CH}), 4.46$ (s, 2H, O-CH $), 1.72$ (s, 3H, C$\left.\mathrm{CH}_{3}\right), 1.08$ (s, 12H, Bpin- $\left.\mathrm{CH}_{3}\right) .{ }^{11} \mathrm{~B}\left\{{ }^{1} \mathrm{H}\right\}$ NMR $\left(128.41 \mathrm{MHz}, 24{ }^{\circ} \mathrm{C}, \mathrm{C}_{6} \mathrm{D}_{6}, \mathrm{ppm}\right): \delta=21.9$. 


\section{Characterization of compound 5r:}<smiles>N#Cc1ccc(CO[R16](=O)c2ccccc2)cc1</smiles>

${ }^{1} \mathrm{H}$ NMR (395.9 MHz, $\left.24{ }^{\circ} \mathrm{C}, \mathrm{C}_{6} \mathrm{D}_{6}, \mathrm{ppm}\right): \delta=6.99\left(\mathrm{~d}, 2 \mathrm{H}, \mathrm{Ar}-H,{ }^{3} J_{\mathrm{H}, \mathrm{H}}=4.6 \mathrm{~Hz}\right), 6.87(\mathrm{~d}$, $\left.2 \mathrm{H}, \mathrm{Ar}-H,{ }^{3} J_{\mathrm{H}, \mathrm{H}}=5.4 \mathrm{~Hz}\right), 4.67\left(\mathrm{~s}, 2 \mathrm{H}, \mathrm{O}-\mathrm{CH}_{2}\right), 1.04$ (s, 12H, Bpin-CH$) .{ }^{11} \mathrm{~B}\left\{{ }^{1} \mathrm{H}\right\} \mathrm{NMR}$ (128.41 MHz, $\left.24{ }^{\circ} \mathrm{C}, \mathrm{C}_{6} \mathrm{D}_{6}, \mathrm{ppm}\right): \delta=21.8$.

\section{Characterization of compound $5 \mathrm{~s}$ :}

BpinO<smiles>CCc1cccc2cnccc12</smiles>

${ }^{1} \mathrm{H}$ NMR $\left(395.9 \mathrm{MHz}, 24{ }^{\circ} \mathrm{C}, \mathrm{C}_{6} \mathrm{D}_{6}, \mathrm{ppm}\right): \delta=9.17(\mathrm{~s}, 1 \mathrm{H}, \mathrm{Ar}-H), 8.54\left(\mathrm{~d}, 1 \mathrm{H}, \mathrm{Ar}-H,{ }^{3} J_{\mathrm{H}, \mathrm{H}}=\right.$ $5.9 \mathrm{~Hz}), 7.64\left(\mathrm{~d}, 1 \mathrm{H}, \mathrm{Ar}-H,{ }^{3} J_{\mathrm{H}, \mathrm{H}}=7.2 \mathrm{~Hz}\right), 7.43\left(\mathrm{~d}, 1 \mathrm{H}, \mathrm{Ar}-H,{ }^{3} J_{\mathrm{H}, \mathrm{H}}=5.9 \mathrm{~Hz}\right), 7.35(\mathrm{~d}, 1 \mathrm{H}$, $\left.\operatorname{Ar}-H,{ }^{3} J_{\mathrm{H}, \mathrm{H}}=8.2 \mathrm{~Hz}\right), 7.10\left(\mathrm{t}, 1 \mathrm{H}, \mathrm{Ar}-H,{ }^{3} J_{\mathrm{H}, \mathrm{H}}=7.5 \mathrm{~Hz}\right), 5.23\left(\mathrm{~s}, 2 \mathrm{H}, \mathrm{O}-\mathrm{CH}_{2}\right), 1.05(\mathrm{~s}, 12 \mathrm{H}$, Bpin- $\left.\mathrm{CH}_{3}\right) .{ }^{11} \mathrm{~B}\left\{{ }^{1} \mathrm{H}\right\}$ NMR $\left(128.41 \mathrm{MHz}, 24{ }^{\circ} \mathrm{C}, \mathrm{C}_{6} \mathrm{D}_{6}, \mathrm{ppm}\right): \delta=21.9$.

\section{Characterization of compound $5 \mathrm{t}$ :}<smiles>CC(=O)c1ccc(COc2ccccc2)cc1</smiles>

${ }^{1} \mathrm{H}$ NMR $\left(395.9 \mathrm{MHz}, 24{ }^{\circ} \mathrm{C}, \mathrm{C}_{6} \mathrm{D}_{6}, \mathrm{ppm}\right): \delta=7.75\left(\mathrm{~d}, 2 \mathrm{H}, \mathrm{Ar}-H,{ }^{3} J_{\mathrm{H}, \mathrm{H}}=8.2 \mathrm{~Hz}\right), 7.21(\mathrm{~d}$, $\left.2 \mathrm{H}, \mathrm{Ar}-\mathrm{H},{ }^{3} J_{\mathrm{H}, \mathrm{H}}=8.6 \mathrm{~Hz}\right), 4.88\left(\mathrm{~s}, 2 \mathrm{H}, \mathrm{O}-\mathrm{CH}_{2}\right), 2.08$ (s, 3H, C-CH3), 1.05 (s 12H, Bpin$\left.\mathrm{CH}_{3}\right) .{ }^{11} \mathrm{~B}\left\{{ }^{1} \mathrm{H}\right\}$ NMR $\left(128.41 \mathrm{MHz}, 24{ }^{\circ} \mathrm{C}, \mathrm{C}_{6} \mathrm{D}_{6}, \mathrm{ppm}\right): \delta=21.9$.

\section{Characterization of compound 7a:}<smiles>CC(O)c1ccccc1</smiles>

${ }^{1} \mathrm{H}$ NMR $\left(395.9 \mathrm{MHz}, 24{ }^{\circ} \mathrm{C}, \mathrm{C}_{6} \mathrm{D}_{6}, \mathrm{ppm}\right): \delta=7.37$ (d, 2H, Ar- $\left.H,{ }^{3} J_{\mathrm{H}, \mathrm{H}}=7.7 \mathrm{~Hz}\right), 7.16-7.12$ $\left(\mathrm{m}, 2 \mathrm{H}\right.$ [overlap with $\mathrm{C}_{6} \mathrm{D}_{6}$ ], Ar- $\left.H\right), 7.07-7.03(\mathrm{~m}, 1 \mathrm{H}, \mathrm{Ar}-H), 5.42\left(\mathrm{q}, 1 \mathrm{H}, \mathrm{O}-\mathrm{CH},{ }^{3} J_{\mathrm{H}, \mathrm{H}}=\right.$ $6.5 \mathrm{~Hz}), 1.46\left(\mathrm{~d}, 3 \mathrm{H}, \mathrm{OCH}-\mathrm{CH}_{3},{ }^{3} \mathrm{~J}_{\mathrm{H}, \mathrm{H}}=6.3 \mathrm{~Hz}\right), 1.02\left(\mathrm{~s}, 6 \mathrm{H}, \mathrm{Bpin}-\mathrm{CH}_{3}\right), 1.00$ (s, 6H, Bpin$\left.\mathrm{CH}_{3}\right) .{ }^{11} \mathrm{~B}\left\{{ }^{1} \mathrm{H}\right\}$ NMR $\left(128.41 \mathrm{MHz}, 24{ }^{\circ} \mathrm{C}, \mathrm{C}_{6} \mathrm{D}_{6}, \mathrm{ppm}\right): \delta=21.7$.

\section{Characterization of compound 7b:}<smiles>CC(Oc1ccccc1)c1ccc2ccccc2c1</smiles>

${ }^{1} \mathrm{H}$ NMR (395.9 MHz, $\left.24{ }^{\circ} \mathrm{C}, \mathrm{C}_{6} \mathrm{D}_{6}, \mathrm{ppm}\right): \delta=7.85$ (s, $\left.1 \mathrm{H}, \mathrm{Ar}-H\right), 7.62-7.59(\mathrm{~m}, 3 \mathrm{H}, \mathrm{Ar}-H)$, $7.47\left(\mathrm{dd}, 1 \mathrm{H}, \operatorname{Ar}-H,{ }^{3} J_{\mathrm{H}, \mathrm{H}}=1.8,8.6 \mathrm{~Hz}\right), 7.26-7.20(\mathrm{~m}, 2 \mathrm{H}, \mathrm{Ar}-H), 5.58\left(\mathrm{q}, 1 \mathrm{H}, \mathrm{O}-\mathrm{CH},{ }^{3} J_{\mathrm{H}, \mathrm{H}}\right.$ $=6.3 \mathrm{~Hz}), 1.55\left(\mathrm{~d}, 3 \mathrm{H}, \mathrm{OCH}-\mathrm{CH}_{3},{ }^{3} J_{\mathrm{H}, \mathrm{H}}=6.4 \mathrm{~Hz}\right), 1.03\left(\mathrm{~s}, 6 \mathrm{H}, \mathrm{Bpin}-\mathrm{CH}_{3}\right), 1.00(\mathrm{~s}, 6 \mathrm{H}, \mathrm{Bpin}-$ $\left.\mathrm{CH}_{3}\right) .{ }^{11} \mathrm{~B}\left\{{ }^{1} \mathrm{H}\right\}$ NMR $\left(128.41 \mathrm{MHz}, 24{ }^{\circ} \mathrm{C}, \mathrm{C}_{6} \mathrm{D}_{6}, \mathrm{ppm}\right): \delta=21.6$. 


\section{Characterization of compound 7c:}<smiles>CC(O[GaH2])c1cccc2ccccc12</smiles>

${ }^{1} \mathrm{H}$ NMR (395.9 MHz, $\left.24{ }^{\circ} \mathrm{C}, \mathrm{C}_{6} \mathrm{D}_{6}, \mathrm{ppm}\right): \delta=7.97-7.95(\mathrm{~m}, 1 \mathrm{H}, \mathrm{Ar}-H), 7.88(\mathrm{~d}, 1 \mathrm{H}, \mathrm{Ar}-H$, $\left.{ }^{3} J_{\mathrm{H}, \mathrm{H}}=7.2 \mathrm{~Hz}\right), 7.64-7.62(\mathrm{~m}, 1 \mathrm{H}, \mathrm{Ar}-H), 7.54\left(\mathrm{~d}, 1 \mathrm{H}, \mathrm{Ar}-H,{ }^{3} J_{\mathrm{H}, \mathrm{H}}=8.2 \mathrm{~Hz}\right), 7.30-7.26$ $(\mathrm{m}, 1 \mathrm{H}, \mathrm{Ar}-H), 7.25-7.20(\mathrm{~m}, 2 \mathrm{H}, \mathrm{Ar}-H), 6.19\left(\mathrm{q}, 1 \mathrm{H}, \mathrm{O}-\mathrm{CH},{ }^{3} \mathrm{~J}_{\mathrm{H}, \mathrm{H}}=6.3 \mathrm{~Hz}\right), 1.63(\mathrm{~d}, 3 \mathrm{H}$, $\left.\mathrm{OCH}-\mathrm{CH}_{3},{ }^{3} \mathrm{~J}_{\mathrm{H}, \mathrm{H}}=6.3 \mathrm{~Hz}\right), 1.02\left(\mathrm{~s}, 6 \mathrm{H}, \mathrm{Bpin}-\mathrm{CH}_{3}\right), 0.98\left(\mathrm{~s}, 6 \mathrm{H}, \mathrm{Bpin}-\mathrm{CH}_{3}\right) .{ }^{11} \mathrm{~B}\left\{{ }^{1} \mathrm{H}\right\} \mathrm{NMR}$ (128.41 MHz, $\left.24{ }^{\circ} \mathrm{C}, \mathrm{C}_{6} \mathrm{D}_{6}, \mathrm{ppm}\right): \delta=21.7$.

\section{Characterization of compound 7d:}

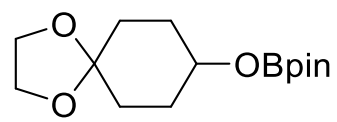

${ }^{1} \mathrm{H}$ NMR (395.9 MHz, $\left.24{ }^{\circ} \mathrm{C}, \mathrm{C}_{6} \mathrm{D}_{6}, \mathrm{ppm}\right): \delta=4.35-4.29(\mathrm{~m}, 1 \mathrm{H}, \mathrm{O}-\mathrm{CH}), 3.53-3.46(\mathrm{~m}$, $\left.4 \mathrm{H}, \mathrm{O}-\mathrm{CH}_{2}\right), 1.92-1.85(\mathrm{~m}, 6 \mathrm{H}, \mathrm{Cy}-\mathrm{H}), 1.58-1.47(\mathrm{~m}, 2 \mathrm{H}, \mathrm{Cy}-\mathrm{H}), 1.06$ (s, 12H, Bpin- $\left.\mathrm{CH}_{3}\right)$. ${ }^{11} \mathrm{~B}\left\{{ }^{1} \mathrm{H}\right\} \mathrm{NMR}\left(128.41 \mathrm{MHz}, 24{ }^{\circ} \mathrm{C}, \mathrm{C}_{6} \mathrm{D}_{6}, \mathrm{ppm}\right): \delta=21.3$.

\section{Characterization of compound 7e:}<smiles>CC(C)C(C)O[PbH2]</smiles>

${ }^{1} \mathrm{H}$ NMR $\left(395.9 \mathrm{MHz}, 24{ }^{\circ} \mathrm{C}, \mathrm{C}_{6} \mathrm{D}_{6}, \mathrm{ppm}\right): \delta=4.15$ (dq, $\left.1 \mathrm{H}, \mathrm{O}-\mathrm{CH},{ }^{3} J_{\mathrm{H}, \mathrm{H}}=6.1 \mathrm{~Hz}\right), 1.69-$ $1.61\left(\mathrm{~m}, 1 \mathrm{H},\left(\mathrm{CH}_{3}\right)_{2} \mathrm{CH}\right), 1.16\left(\mathrm{~d}, 3 \mathrm{H},-\mathrm{CH}_{3},{ }^{3} \mathrm{~J}_{\mathrm{H}, \mathrm{H}}=6.3 \mathrm{~Hz}\right), 1.07\left(\mathrm{~s}, 12 \mathrm{H}, \mathrm{Bpin}-\mathrm{CH}_{3}\right), 0.92$ $\left(\mathrm{d}, 3 \mathrm{H},-\mathrm{CH}_{3},{ }^{3} J_{\mathrm{H}, \mathrm{H}}=6.4 \mathrm{~Hz}\right), 0.86\left(\mathrm{~d}, 3 \mathrm{H},-\mathrm{CH}_{3},{ }^{3} \mathrm{~J}_{\mathrm{H}, \mathrm{H}}=6.8 \mathrm{~Hz}\right) .{ }^{11} \mathrm{~B}\left\{{ }^{1} \mathrm{H}\right\} \mathrm{NMR}(128.41$ $\left.\mathrm{MHz}, 24^{\circ} \mathrm{C}, \mathrm{C}_{6} \mathrm{D}_{6}, \mathrm{ppm}\right): \delta=21.5$.

\section{Characterization of compound 7f:}<smiles>CC(O[R18](=O)c1ccccc1)c1cccs1</smiles>

${ }^{1} \mathrm{H}$ NMR (395.9 MHz, $\left.24{ }^{\circ} \mathrm{C}, \mathrm{C}_{6} \mathrm{D}_{6}, \mathrm{ppm}\right): \delta=6.85\left(\mathrm{dd}, 1 \mathrm{H}, \mathrm{Ar}-H,{ }^{3} J_{\mathrm{H}, \mathrm{H}}=0.9,3.6 \mathrm{~Hz}\right), 6.82$ $\left(\mathrm{dd}, 1 \mathrm{H}, \operatorname{Ar}-H,{ }^{3} J_{\mathrm{H}, \mathrm{H}}=0.9,5.0 \mathrm{~Hz}\right), 6.69\left(\mathrm{dd}, 1 \mathrm{H}, \mathrm{Ar}-H,{ }^{3} J_{\mathrm{H}, \mathrm{H}}=3.6,5.0 \mathrm{~Hz}\right), 5.64(\mathrm{q}, 1 \mathrm{H}, \mathrm{O}-$ $\left.\mathrm{CH},{ }^{3} J_{\mathrm{H}, \mathrm{H}}=6.3 \mathrm{~Hz}\right), 1.52\left(\mathrm{~d}, 3 \mathrm{H}, \mathrm{OCH}-\mathrm{CH}_{3},{ }^{3} J_{\mathrm{H}, \mathrm{H}}=6.3 \mathrm{~Hz}\right), 1.03\left(\mathrm{~s}, 12 \mathrm{H}, \mathrm{Bpin}-\mathrm{CH}_{3}\right)$.

${ }^{11} \mathrm{~B}\left\{{ }^{1} \mathrm{H}\right\}$ NMR $\left(128.41 \mathrm{MHz}, 24{ }^{\circ} \mathrm{C}, \mathrm{C}_{6} \mathrm{D}_{6}, \mathrm{ppm}\right): \delta=21.7$.

\section{Characterization of compound 7g:}<smiles>CC(OCc1ccccc1)c1ccccn1</smiles>

${ }^{1} \mathrm{H}$ NMR $\left(395.9 \mathrm{MHz}, 24{ }^{\circ} \mathrm{C}, \mathrm{C}_{6} \mathrm{D}_{6}, \mathrm{ppm}\right): \delta=8.38\left(\mathrm{~d}, 1 \mathrm{H}, \mathrm{Ar}-H,{ }^{3} J_{\mathrm{H}, \mathrm{H}}=5.0 \mathrm{~Hz}\right), 7.30(\mathrm{~d}$, $\left.1 \mathrm{H}, \operatorname{Ar}-H,{ }^{3} J_{\mathrm{H}, \mathrm{H}}=7.7 \mathrm{~Hz}\right), 7.15-7.10(\mathrm{~m}, 1 \mathrm{H}, \mathrm{Ar}-H), 6.64-6.61(\mathrm{~m}, 1 \mathrm{H}, \operatorname{Ar}-H), 5.54(\mathrm{q}, 1 \mathrm{H}$, $\left.\mathrm{O}-\mathrm{CH},{ }^{3} J_{\mathrm{H}, \mathrm{H}}=6.7 \mathrm{~Hz}\right), 1.60\left(\mathrm{~d}, 3 \mathrm{H}, \mathrm{OCH}-\mathrm{CH}_{3},{ }^{3} J_{\mathrm{H}, \mathrm{H}}=6.3 \mathrm{~Hz}\right), 1.13\left(\mathrm{~s}, 6 \mathrm{H}, \mathrm{Bpin}-\mathrm{CH}_{3}\right), 1.11$ (s, 6H, Bpin- $\left.\mathrm{CH}_{3}\right) .{ }^{11} \mathrm{~B}\left\{{ }^{1} \mathrm{H}\right\}$ NMR $\left(128.41 \mathrm{MHz}, 24{ }^{\circ} \mathrm{C}, \mathrm{C}_{6} \mathrm{D}_{6}, \mathrm{ppm}\right): \delta=19.5$. 


\section{Characterization of compound 7h:}<smiles>CC(OCCCOc1ccccc1)c1ccc(Br)cc1</smiles>

${ }^{1} \mathrm{H}$ NMR $\left(395.9 \mathrm{MHz}, 24{ }^{\circ} \mathrm{C}, \mathrm{C}_{6} \mathrm{D}_{6}, \mathrm{ppm}\right): \delta 7.23\left(\mathrm{~d}, 2 \mathrm{H}, \mathrm{Ar}-H,{ }^{3} J_{\mathrm{H}, \mathrm{H}}=8.6 \mathrm{~Hz}\right), 6.99,(\mathrm{~d}, 2 \mathrm{H}$, $\left.\mathrm{Ar}-H,{ }^{3} J_{\mathrm{H}, \mathrm{H}}=8.2 \mathrm{~Hz}\right), 5.23\left(\mathrm{q}, 1 \mathrm{H}, \mathrm{O}-\mathrm{CH},{ }^{3} J_{\mathrm{H}, \mathrm{H}}=6.5 \mathrm{~Hz}\right), 1.33\left(\mathrm{~d}, 3 \mathrm{H}, \mathrm{OCH}-\mathrm{CH}_{3},{ }^{3} J_{\mathrm{H}, \mathrm{H}}=6.4\right.$ $\mathrm{Hz}), 1.02$ (s, 6H, Bpin- $\left.\mathrm{CH}_{3}\right), 0.99$ (s, 6H, Bpin- $\left.\mathrm{CH}_{3}\right) .{ }^{11} \mathrm{~B}\left\{{ }^{1} \mathrm{H}\right\} \mathrm{NMR}\left(128.41 \mathrm{MHz}, 24{ }^{\circ} \mathrm{C}\right.$, $\left.\mathrm{C}_{6} \mathrm{D}_{6}, \mathrm{ppm}\right): \delta=21.5$.

\section{Characterization of compound $7 \mathbf{j}$ :}<smiles>CCCCCCOC(C)c1ccc(C#N)cc1</smiles>

${ }^{1} \mathrm{H}$ NMR $\left(395.9 \mathrm{MHz}, 24{ }^{\circ} \mathrm{C}, \mathrm{C}_{6} \mathrm{D}_{6}, \mathrm{ppm}\right): \delta=7.02-6.94(\mathrm{~m}, 4 \mathrm{H}, \mathrm{Ar}-H), 5.15$ (q, 1H, O-CH, $\left.{ }^{3} J_{\mathrm{H}, \mathrm{H}}=6.5 \mathrm{~Hz}\right), 1.23\left(\mathrm{~d}, 3 \mathrm{H}, \mathrm{OCH}-\mathrm{CH}_{3},{ }^{3} J_{\mathrm{H}, \mathrm{H}}=6.3 \mathrm{~Hz}\right), 1.02\left(\mathrm{~s}, 6 \mathrm{H}, \mathrm{Bpin}-\mathrm{CH}_{3}\right), 0.99(\mathrm{~s}, 6 \mathrm{H}$, Bpin- $\left.\mathrm{CH}_{3}\right) .{ }^{11} \mathrm{~B}\left\{{ }^{1} \mathrm{H}\right\} \mathrm{NMR}\left(128.41 \mathrm{MHz}, 24{ }^{\circ} \mathrm{C}, \mathrm{C}_{6} \mathrm{D}_{6}, \mathrm{ppm}\right): \delta=21.6$.

\section{Characterization of compound 9a:}<smiles>Brc1ccccc1</smiles>

${ }^{1} \mathrm{H}$ NMR $\left(395.9 \mathrm{MHz}, 24{ }^{\circ} \mathrm{C}, \mathrm{C}_{6} \mathrm{D}_{6}, \mathrm{ppm}\right): \delta=6.52-6.50(\mathrm{~m}, 2 \mathrm{H}, \mathrm{N}=\mathrm{CH}), 4.59-4.55(\mathrm{~m}$, $2 \mathrm{H}, \mathrm{C}=\mathrm{CH}), 2.83-2.81\left(\mathrm{~m}, 2 \mathrm{H},-\mathrm{CH}_{2}\right), 0.98\left(\mathrm{~s}, 12 \mathrm{H}, \mathrm{Bpin}-\mathrm{CH}_{3}\right) .{ }^{11} \mathrm{~B}\left\{{ }^{1} \mathrm{H}\right\} \mathrm{NMR}(128.41$ $\left.\mathrm{MHz}, 24^{\circ} \mathrm{C}, \mathrm{C}_{6} \mathrm{D}_{6}, \mathrm{ppm}\right): \delta=23.1$.

\section{Characterization of compound 9b:}<smiles>COC(=O)C1=CN(c2ccccc2)C=CC1</smiles>

${ }^{1} \mathrm{H}$ NMR $\left(395.9 \mathrm{MHz}, 24{ }^{\circ} \mathrm{C}, \mathrm{C}_{6} \mathrm{D}_{6}, \mathrm{ppm}\right): \delta=7.83$ (s, $\left.1 \mathrm{H}, \mathrm{N}=\mathrm{CH}\right), 6.33\left(\mathrm{~d}, 1 \mathrm{H}, \mathrm{N}=\mathrm{CH},{ }^{3} J_{\mathrm{H}, \mathrm{H}}\right.$ $=6.8 \mathrm{~Hz}), 4.70-4.68(\mathrm{~m}, 1 \mathrm{H}, \mathrm{C}=\mathrm{CH}), 3.40\left(\mathrm{~s}, 3 \mathrm{H}, \mathrm{O}-\mathrm{CH}_{3}\right), 3.20\left(\mathrm{~s}, 2 \mathrm{H},-\mathrm{CH}_{2}\right), 0.93(\mathrm{~s}, 12 \mathrm{H}$, Bpin- $\left.\mathrm{CH}_{3}\right) .{ }^{11} \mathrm{~B}\left\{{ }^{1} \mathrm{H}\right\}$ NMR $\left(128.41 \mathrm{MHz}, 24{ }^{\circ} \mathrm{C}, \mathrm{C}_{6} \mathrm{D}_{6}, \mathrm{ppm}\right): \delta=23.1$.

\section{Characterization of compound 9c:}<smiles>N#CC1=CN(c2ccccc2)C=CC1</smiles>

${ }^{1} \mathrm{H}$ NMR $\left(395.9 \mathrm{MHz}, 24{ }^{\circ} \mathrm{C}, \mathrm{C}_{6} \mathrm{D}_{6}, \mathrm{ppm}\right): \delta=6.82\left(\mathrm{~d}, 1 \mathrm{H}, \mathrm{N}=\mathrm{CH},{ }^{3} J_{\mathrm{H}, \mathrm{H}}=0.9 \mathrm{~Hz}\right), 6.05(\mathrm{dq}$, $\left.1 \mathrm{H}, \mathrm{N}=\mathrm{CH},{ }^{3} \mathrm{~J}_{\mathrm{H}, \mathrm{H}}=1.7,8.2 \mathrm{~Hz}\right), 4.30\left(\mathrm{dt}, 1 \mathrm{H}, \mathrm{C}=\mathrm{CH},{ }^{3} J_{\mathrm{H}, \mathrm{H}}=3.4,8.2 \mathrm{~Hz}\right), 2.62\left(\mathrm{t}, 2 \mathrm{H},-\mathrm{CH}_{2}\right.$, $\left.{ }^{3} J_{\mathrm{H}, \mathrm{H}}=1.4 \mathrm{~Hz}\right), 0.94\left(\mathrm{~s}, 12 \mathrm{H}, \mathrm{Bpin}-\mathrm{CH}_{3}\right) .{ }^{11} \mathrm{~B}\left\{{ }^{1} \mathrm{H}\right\} \mathrm{NMR}\left(128.41 \mathrm{MHz}, 24{ }^{\circ} \mathrm{C}, \mathrm{C}_{6} \mathrm{D}_{6}, \mathrm{ppm}\right): \delta$ $=22.7$. 


\section{Characterization of compound 9d:}<smiles>Brc1ccccc1</smiles>

${ }^{1} \mathrm{H}$ NMR $\left(395.9 \mathrm{MHz}, 24{ }^{\circ} \mathrm{C}, \mathrm{C}_{6} \mathrm{D}_{6}, \mathrm{ppm}\right): \delta=7.80\left(\mathrm{~d}, 1 \mathrm{H}, \mathrm{N}=\mathrm{CH},{ }^{3} J_{\mathrm{H}, \mathrm{H}}=8.2 \mathrm{~Hz}\right), 7.11-$ $7.07(\mathrm{~m}, 1 \mathrm{H}, \mathrm{Ar}-H), 6.86-6.78(\mathrm{~m}, 2 \mathrm{H}, \mathrm{Ar}-H), 6.26\left(\mathrm{~d}, 1 \mathrm{H}, \mathrm{Ar}-H,{ }^{3} J_{\mathrm{H}, \mathrm{H}}=9.1 \mathrm{~Hz}\right), 5.60-$ $5.56(\mathrm{~m}, 1 \mathrm{H}, \mathrm{C}=\mathrm{CH}), 4.16-4.15\left(\mathrm{~m}, 2 \mathrm{H},-\mathrm{CH}_{2}\right), 1.04\left(\mathrm{~s}, 12 \mathrm{H}, \mathrm{Bpin}-\mathrm{CH}_{3}\right) .{ }^{11} \mathrm{~B}\left\{{ }^{1} \mathrm{H}\right\} \mathrm{NMR}$ $\left(128.41 \mathrm{MHz}, 24{ }^{\circ} \mathrm{C}, \mathrm{C}_{6} \mathrm{D}_{6}, \mathrm{ppm}\right): \delta=23.0$.

\section{Characterization of compound 9e:}<smiles>Brc1cccnn1</smiles>

${ }^{1} \mathrm{H}$ NMR $\left(395.9 \mathrm{MHz}, 24{ }^{\circ} \mathrm{C}, \mathrm{C}_{6} \mathrm{D}_{6}, \mathrm{ppm}\right): \delta=6.62-6.61(\mathrm{~m}, 1 \mathrm{H}, \mathrm{N}=\mathrm{CH}), 6.48(\mathrm{~d}, 1 \mathrm{H}$, $\left.\mathrm{N}=\mathrm{CH},{ }^{3} J_{\mathrm{H}, \mathrm{H}}=1.8 \mathrm{~Hz}\right), 4.32-4.30(\mathrm{~m}, 1 \mathrm{H}, \mathrm{C}=\mathrm{CH}), 2.30\left(\mathrm{~s}, 2 \mathrm{H},-\mathrm{CH}_{2}\right), 1.04(\mathrm{~s}, 12 \mathrm{H}, \mathrm{Bpin}-$ $\left.\mathrm{CH}_{3}\right) .{ }^{11} \mathrm{~B}\left\{{ }^{1} \mathrm{H}\right\}$ NMR $\left(128.41 \mathrm{MHz}, 24{ }^{\circ} \mathrm{C}, \mathrm{C}_{6} \mathrm{D}_{6}, \mathrm{ppm}\right): \delta=23.6$.

\section{Characterization of compound 9f:}<smiles>Brc1cccnc1</smiles>

${ }^{1} \mathrm{H}$ NMR $\left(395.9 \mathrm{MHz}, 24{ }^{\circ} \mathrm{C}, \mathrm{C}_{6} \mathrm{D}_{6}, \mathrm{ppm}\right): \delta=8.17\left(\mathrm{~d}, 1 \mathrm{H}, \mathrm{N}=\mathrm{CH},{ }^{3} J_{\mathrm{H}, \mathrm{H}}=4.5 \mathrm{~Hz}\right), 6.28(\mathrm{dt}$, $\left.1 \mathrm{H}, \mathrm{C}=\mathrm{CH},{ }^{3} \mathrm{~J}_{\mathrm{H}, \mathrm{H}}=1.5,4.9 \mathrm{~Hz}\right), 4.82(\mathrm{~s}, 1 \mathrm{H}, \mathrm{N}=\mathrm{CH}), 3.83-3.81\left(\mathrm{~m}, 2 \mathrm{H},-\mathrm{CH}_{2}\right), 1.06(\mathrm{~s}$, $\left.12 \mathrm{H}, \mathrm{Bpin}-\mathrm{CH}_{3}\right) .{ }^{11} \mathrm{~B}\left\{{ }^{1} \mathrm{H}\right\}$ NMR (128.41 MHz, $\left.24{ }^{\circ} \mathrm{C}, \mathrm{C}_{6} \mathrm{D}_{6}, \mathrm{ppm}\right): \delta=23.2$. 
Table S5. Catalytic table for hydroboration of aldehyde derivatives using HBpin. Isolated yields are shown in parentheses.

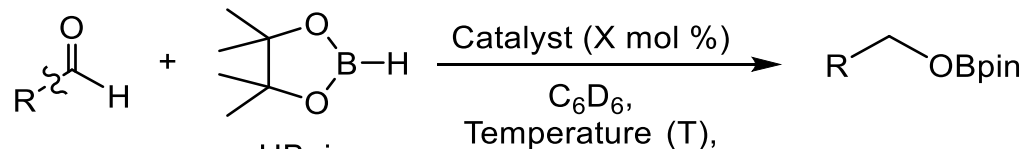

$$
\begin{aligned}
& 4 \text { HBpin Time }(\mathrm{t}) \text {, }
\end{aligned}
$$

\begin{tabular}{|c|c|c|c|c|c|c|c|c|}
\hline Entry & Aldehyde & Product & Cat & $X$ & $\mathrm{~T}\left({ }^{\circ} \mathrm{C}\right)$ & $t(\min )$ & $\begin{array}{l}\text { Yield } \\
(\%)\end{array}$ & $\begin{array}{l}\text { TOF } \\
\left(\mathrm{h}^{-1}\right)\end{array}$ \\
\hline 1 & & & - & - & 24 & 25 & 0 & - \\
\hline 2 & & & $\mathrm{I}_{\mathrm{Me}}$ & 0.1 & 24 & 20 & 23.9 & 717 \\
\hline 3 & & & 1 & 1 & 24 & 25 & 90.1 & 216.2 \\
\hline 4 & & & 1 & 5 & 24 & 20 & 98.0 & 58.8 \\
\hline 5 & & & 1 & 10 & 24 & 10 & $\begin{array}{l}>99 \\
(89)\end{array}$ & 115.8 \\
\hline 6 & & & 1 & 10 & 24 & 10 & $\begin{array}{l}>99 \\
(91)\end{array}$ & 115.8 \\
\hline 7 & & & 1 & 10 & 24 & 10 & $\begin{array}{l}>99 \\
(87)\end{array}$ & 115.8 \\
\hline 8 & & & 1 & 10 & 24 & 10 & $\begin{array}{l}>99 \\
(87)\end{array}$ & 115.8 \\
\hline 9 & & & 1 & 10 & 24 & 10 & $\begin{array}{l}>99 \\
(83)\end{array}$ & 115.8 \\
\hline 10 & & & 1 & 10 & 24 & 10 & $>99$ & 115.8 \\
\hline
\end{tabular}




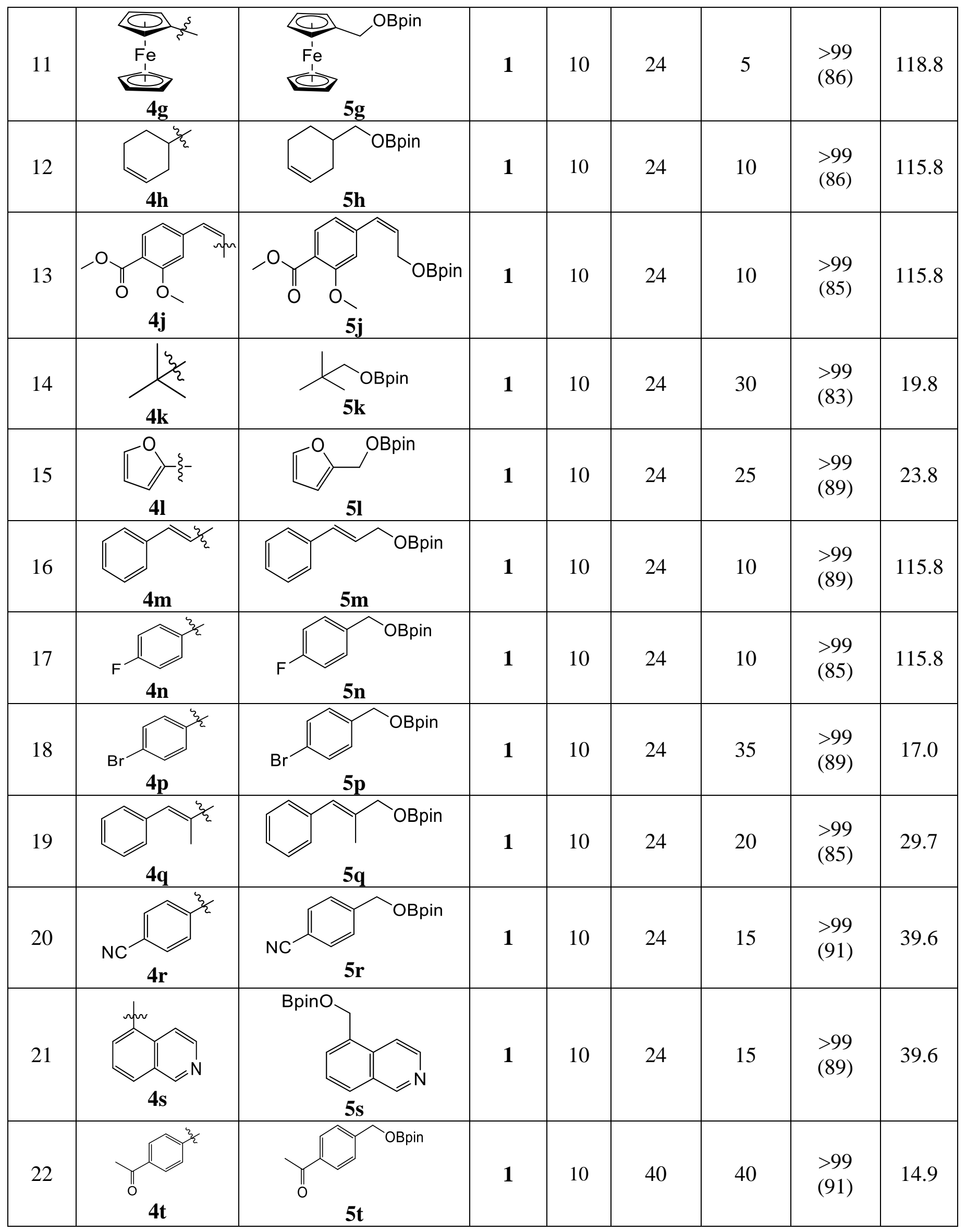


Table S6. Catalytic table for hydroboration of ketone derivatives using HBpin. Isolated yields are shown in parentheses.

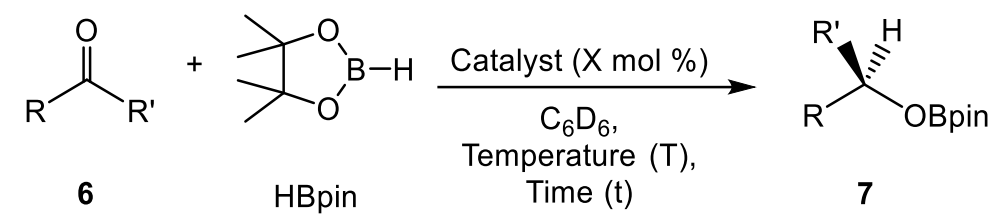

\begin{tabular}{|c|c|c|c|c|c|c|c|c|}
\hline Entry & Ketone & Product & Cat & $X$ & $\begin{array}{c}\mathrm{T} \\
\left({ }^{\circ} \mathrm{C}\right)\end{array}$ & $t(h)$ & $\begin{array}{c}\text { Yield } \\
(\%)\end{array}$ & $\begin{array}{l}\text { TOF } \\
\left(\mathrm{h}^{-1}\right)\end{array}$ \\
\hline 1 & & & - & - & 90 & 24 & 0 & - \\
\hline 2 & & & $\mathrm{I}_{\mathrm{Me}}$ & 0.1 & 90 & 24 & 0 & - \\
\hline 3 & & & 1 & 1 & 90 & 6 & 20.9 & 3.48 \\
\hline 4 & & & 1 & 5 & 90 & 6 & 62.7 & 2.09 \\
\hline 5 & & & 1 & 10 & 90 & 6 & $\begin{array}{l}>99 \\
(84)\end{array}$ & 1.65 \\
\hline 6 & & & 1 & 10 & 90 & 5 & $\begin{array}{l}>99 \\
(90)\end{array}$ & 1.98 \\
\hline 7 & & & 1 & 10 & 90 & 6 & $\begin{array}{l}>99 \\
(86)\end{array}$ & 1.65 \\
\hline 8 & 6d & $7 d$ & 1 & 10 & 90 & 1 & $\begin{array}{l}>99 \\
(85)\end{array}$ & 9.90 \\
\hline
\end{tabular}




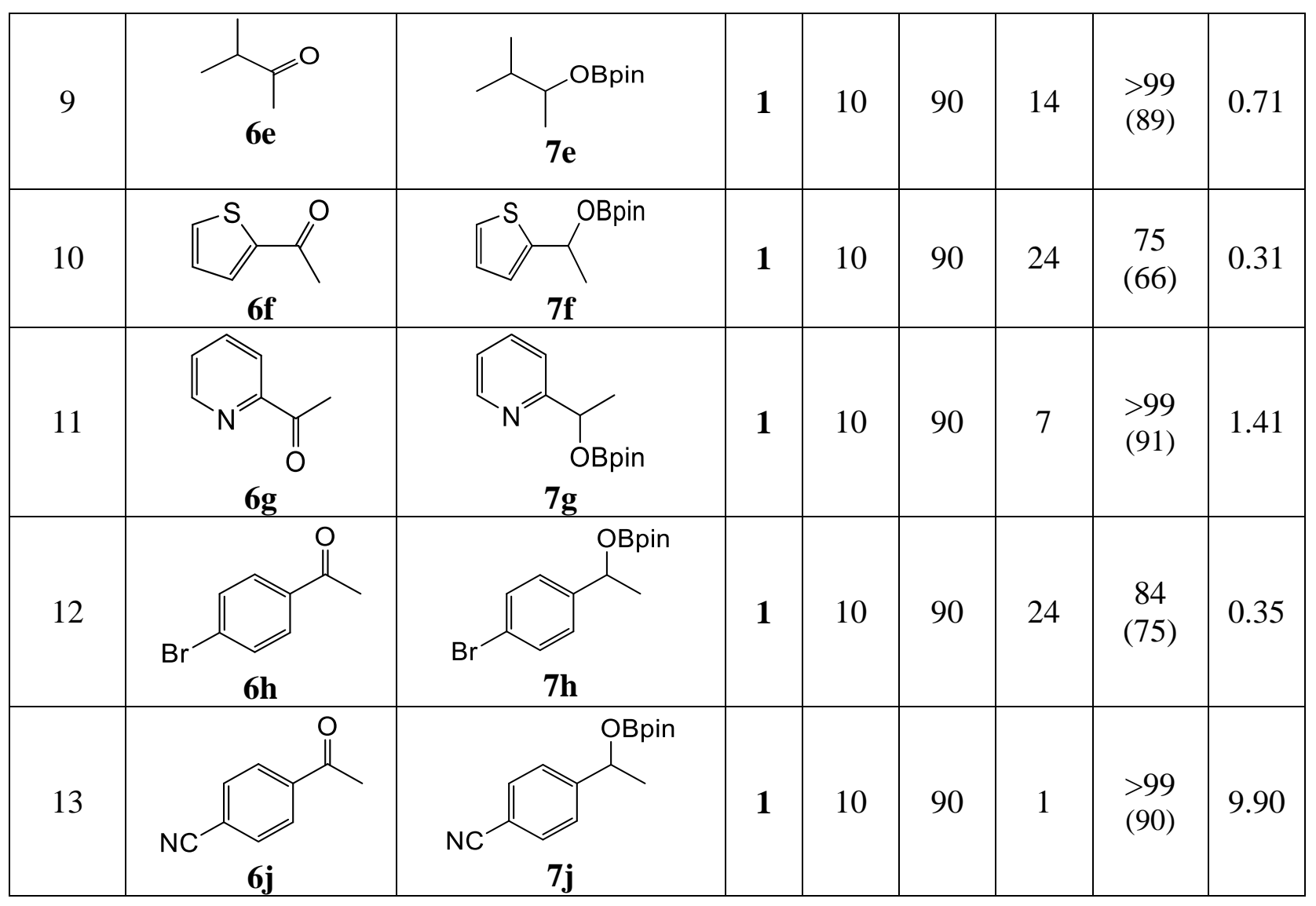


Table S7. Catalytic table for hydroboration of pyridine derivatives using HBpin. Isolated yields are shown in parentheses.

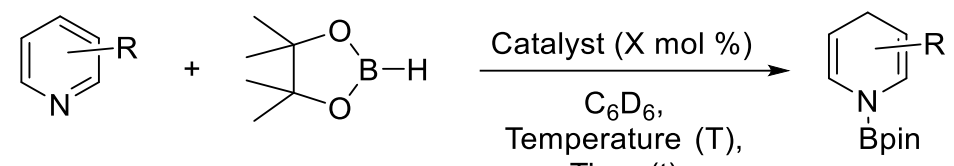

$$
\begin{aligned}
& 8 \text { HBpin } \quad \text { Time (t) } \quad 9
\end{aligned}
$$

\begin{tabular}{|c|c|c|c|c|c|c|c|c|}
\hline Entry & Pyridine & Product & Cat & $X$ & $\begin{array}{c}\mathrm{T} \\
\left({ }^{\circ} \mathrm{C}\right) \\
\end{array}$ & $\mathrm{t}(\mathrm{h})$ & $\begin{array}{c}\text { Yield } \\
(\%)\end{array}$ & $\begin{array}{l}\text { TOF } \\
\left(\mathrm{h}^{-1}\right) \\
\end{array}$ \\
\hline 1 & $8 \mathbf{a}$ & $\begin{array}{l}\text { Bpin } \\
\mathbf{9 a}\end{array}$ & - & - & 90 & 24 & 0 & - \\
\hline 2 & $8 \mathbf{a}$ & $\begin{array}{l}\text { Bpin } \\
\mathbf{9 a}\end{array}$ & $\mathrm{I}_{\mathrm{Me}}$ & 0.1 & 90 & 24 & 0 & - \\
\hline 3 & $8 \mathbf{a}$ & $\begin{array}{l}\text { Bpin } \\
\mathbf{9 a}\end{array}$ & $\mathrm{I}_{\mathrm{Me}}$ & 10 & 90 & 24 & 0 & - \\
\hline 4 & $8 \mathbf{a}$ & $\begin{array}{l}\text { Bpin } \\
\mathbf{9 a}\end{array}$ & $\mathrm{Ph}_{3} \mathrm{SiH}$ & 10 & 24 & 24 & 0 & 0 \\
\hline 5 & $8 \mathbf{a}$ & $\begin{array}{l}\text { Bpin } \\
\mathbf{9 a}\end{array}$ & $(\mathrm{EtO})_{3} \mathrm{SiH}$ & 10 & 24 & 24 & 0 & 0 \\
\hline 6 & $8 \mathbf{a}$ & $\begin{array}{l}\text { Bpin } \\
9 \mathbf{a}\end{array}$ & 1 & 1 & 90 & 4 & 26.8 & 6.7 \\
\hline 7 & $8 \mathbf{a}$ & $\begin{array}{l}\text { Bpin } \\
9 \mathbf{a}\end{array}$ & 1 & 5 & 90 & 4 & 32.5 & 1.63 \\
\hline
\end{tabular}




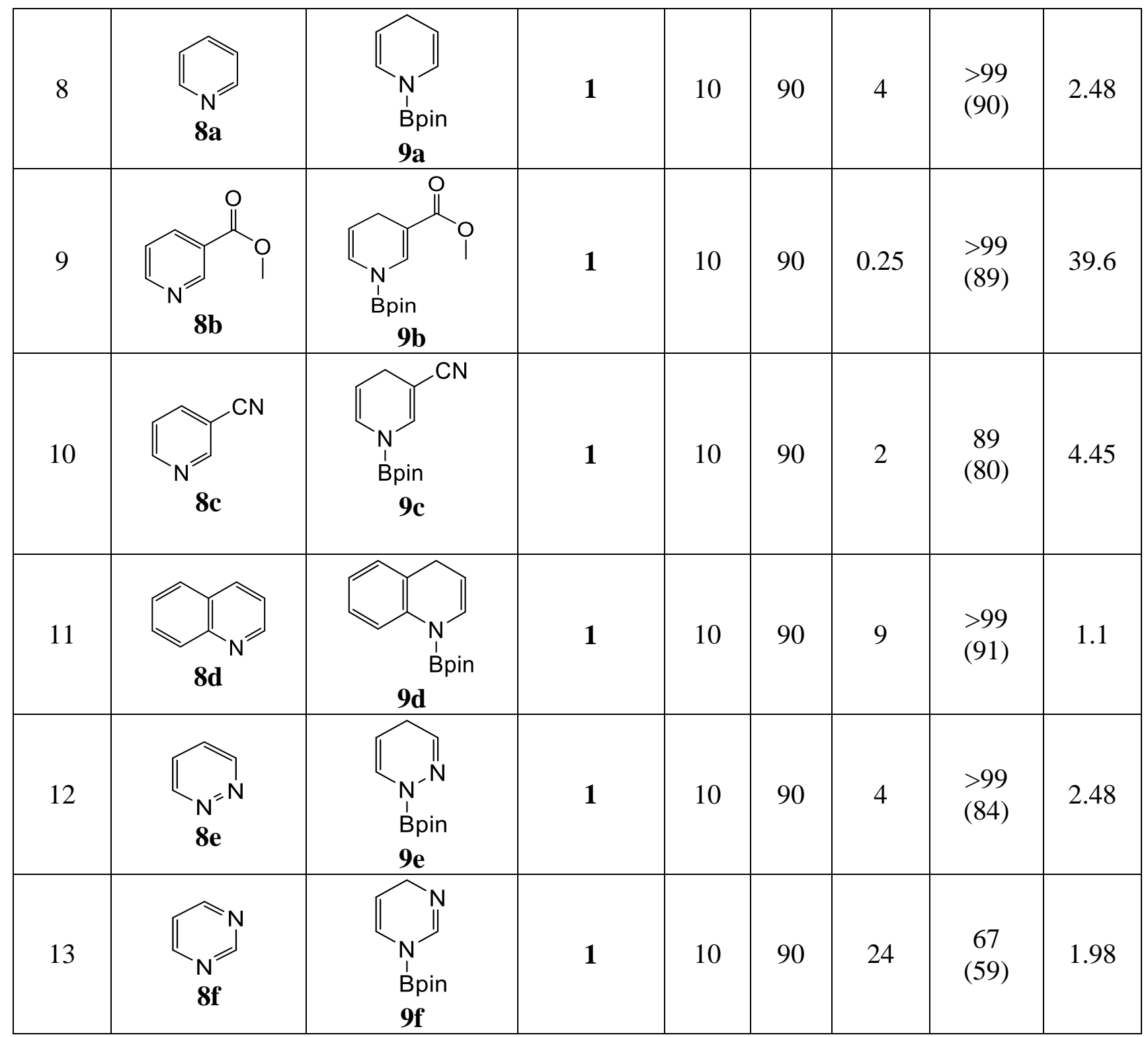

Page S22 
General procedures for the catalytic hydroboration of aldehyde, ketone and pyridine derivatives using 3 as the catalyst:

Catalyst 3 (5.3 mg, $0.01 \mathrm{mmol})$, HBpin $(16.0 \mu \mathrm{L}, 0.11 \mathrm{mmol}, 1.1$ equiv. $)$ and substrates $(0.10$ mmol, 1 equiv.) were mixed with $0.5 \mathrm{~mL}$ of $\mathrm{C}_{6} \mathrm{D}_{6}$ in a J-Young NMR tube at ambient temperature. The reaction mixture was stirred with the reaction conditions stated in Tables S8 - S10. The reactions were monitored by NMR spectroscopy to determine their yields. The chemical shifts of the products agree with the reported values in the literatures. ${ }^{\mathrm{S}}$ All the catalytic trials were repeated in triplicate.

Table S8. Catalytic table for hydroboration of aldehyde derivatives using HBpin.

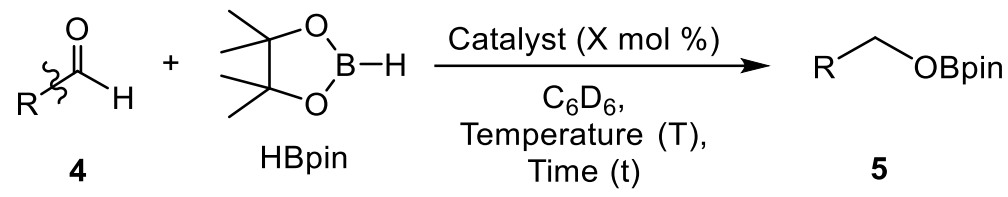

\begin{tabular}{|c|c|c|c|c|c|c|c|c|}
\hline Entry & \multirow{2}{*}{ Aldehyde } & Product & Cat & $\mathrm{X}$ & $\mathrm{T}\left({ }^{\circ} \mathrm{C}\right)$ & $\mathrm{t}(\mathrm{h})$ & $\begin{array}{c}\text { Yield } \\
(\%)\end{array}$ & $\begin{array}{c}\mathrm{TOF} \\
\left(\mathrm{h}^{-1}\right)\end{array}$ \\
\cline { 1 - 8 } 1 & \multirow{4a}{*}{2} & $\mathbf{5 a}$ & $\mathbf{3}$ & 2.5 & 24 & 0.08 & 86 & 430 \\
\cline { 1 - 8 } & & $\mathbf{3}$ & 2.5 & 24 & 0.17 & $>99$ & 235 \\
\hline
\end{tabular}

Table S9. Catalytic table for hydroboration of ketone derivatives using HBpin.

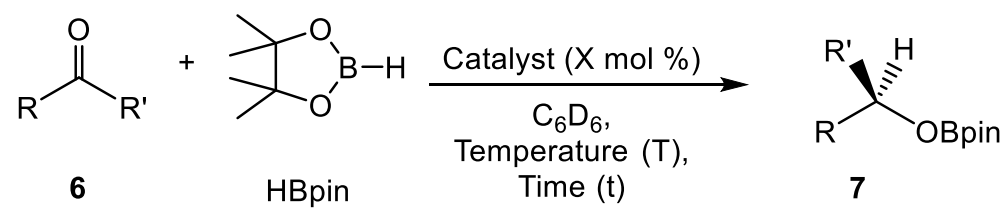

\begin{tabular}{|c|c|c|c|c|c|c|c|c|}
\hline Entry & Ketone & Product & Cat & $\mathrm{X}$ & $\mathrm{T}\left({ }^{\circ} \mathrm{C}\right)$ & $\mathrm{t}(\mathrm{h})$ & $\begin{array}{c}\text { Yield } \\
(\%)\end{array}$ & $\begin{array}{l}\text { TOF } \\
\left(\mathrm{h}^{-1}\right)\end{array}$ \\
\hline \multirow[t]{2}{*}{1} & \multirow{3}{*}{$6 d$} & \multirow{3}{*}{$7 d$} & 3 & 3 & 90 & 0.08 & 8.9 & 37 \\
\hline & & & 3 & 3 & 90 & 0.58 & 31 & 18 \\
\hline \multirow[t]{2}{*}{2} & & & 3 & 5 & 90 & 0.17 & 65 & 77 \\
\hline & & & 3 & 5 & 90 & 1.00 & $>99$ & 20 \\
\hline
\end{tabular}


Table S10. Catalytic table for hydroboration of pyridine derivatives using HBpin.

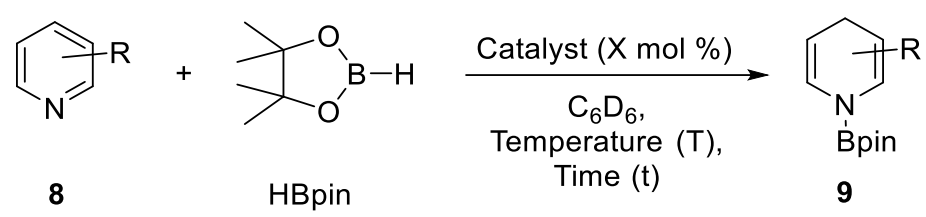

\begin{tabular}{|c|c|c|c|c|c|c|c|c|}
\hline Entry & Pyridine & Product & Cat & $\mathrm{X}$ & $\mathrm{T}\left({ }^{\circ} \mathrm{C}\right)$ & $\mathrm{t}(\mathrm{h})$ & $\begin{array}{c}\text { Yield } \\
(\%)\end{array}$ & $\begin{array}{c}\mathrm{TOF} \\
\left(\mathrm{h}^{-1}\right)\end{array}$ \\
\hline 1 & & $\mathbf{3}$ & 3 & 90 & 1 & 18 & 6.0 \\
& $\mathbf{8 a}$ & $\begin{array}{c}\text { Bpin } \\
\text { Ba }\end{array}$ & $\mathbf{3}$ & 3 & 90 & 3 & 72 & 8.0 \\
& & $\mathbf{9 a}$ & 3 & 90 & 4 & $>99$ & 8.3 \\
\hline
\end{tabular}

\section{S2. Selected spectra}

List of abbreviation used in NMR spectra.

\begin{tabular}{|c|c|}
\hline Abbreviation & Definition \\
\hline Cat & Catalyst \\
HBpin & Pinacolborane \\
IS & Internal standard \\
sm & Starting material (substrate) \\
pdt & Hydroboration product \\
\hline
\end{tabular}




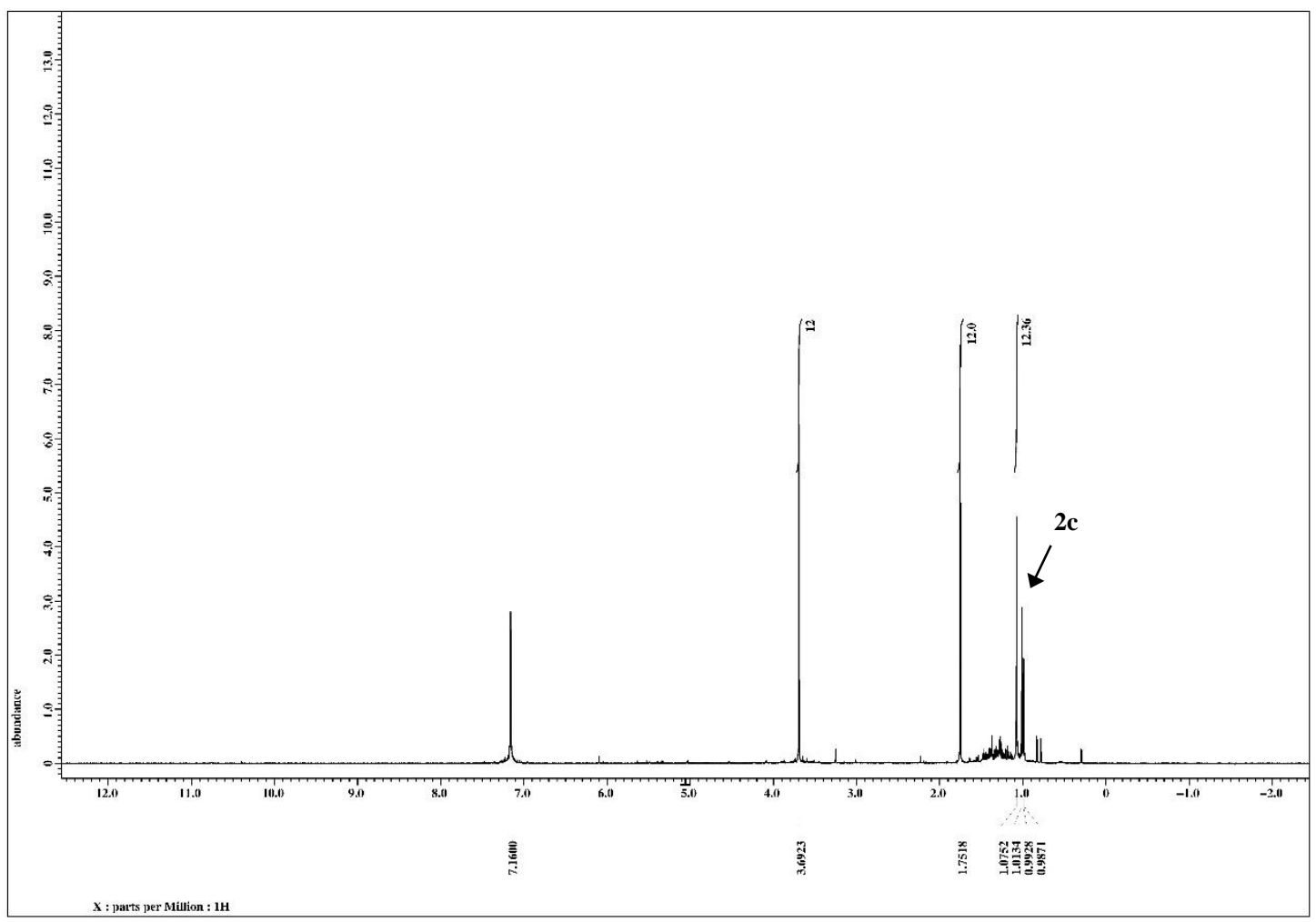

Figure S1. ${ }^{1} \mathrm{H}$ NMR spectrum of compound 3 (in $\mathrm{C}_{6} \mathrm{D}_{6}$ ).

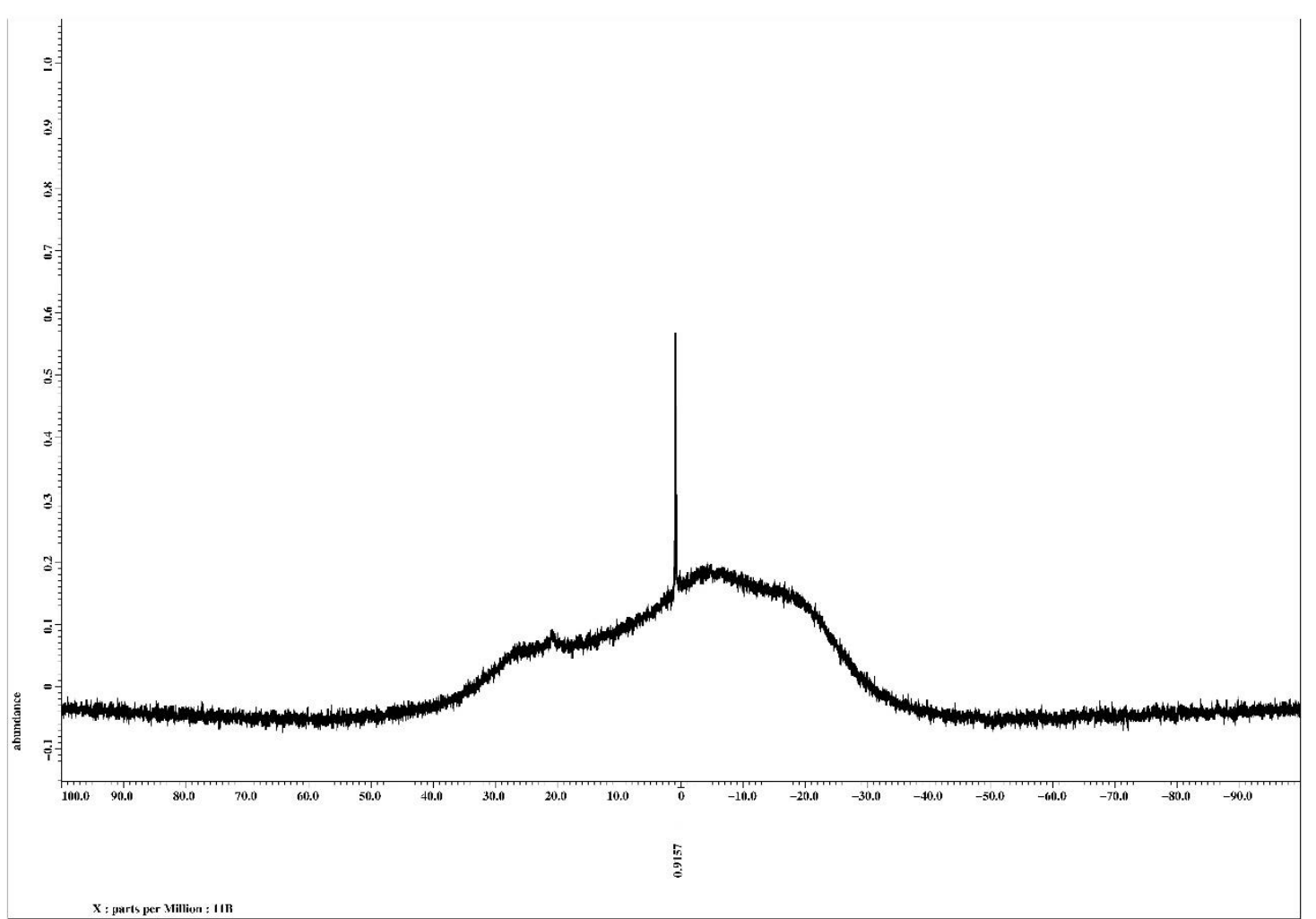

Figure S2. ${ }^{11} \mathrm{~B}$ NMR spectrum of compound 3 (in $\mathrm{C}_{6} \mathrm{D}_{6}$ ). 


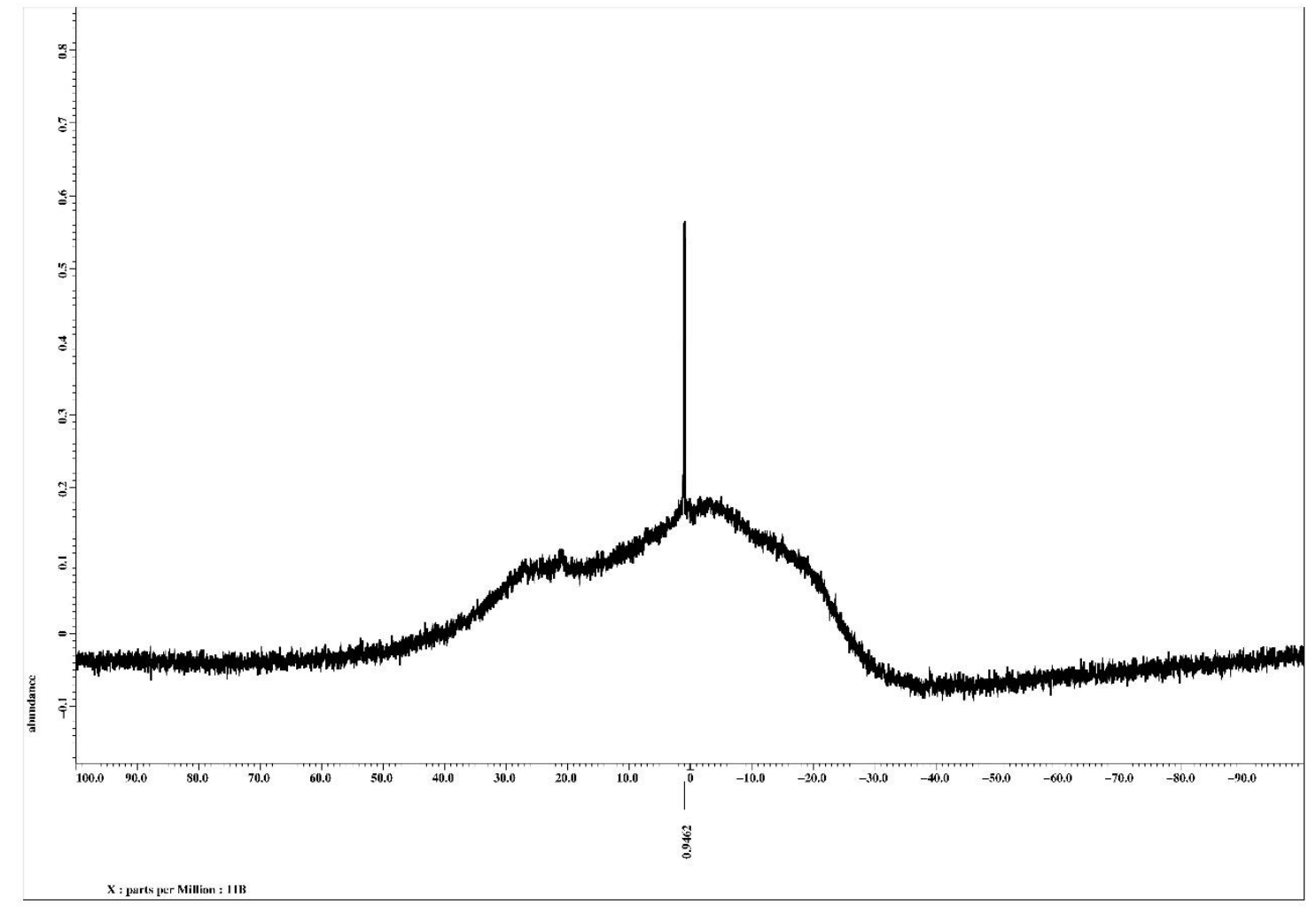

Figure S3. ${ }^{11} \mathrm{~B}\left\{{ }^{1} \mathrm{H}\right\}$ NMR spectrum of compound 3 (in $\mathrm{C}_{6} \mathrm{D}_{6}$ ).

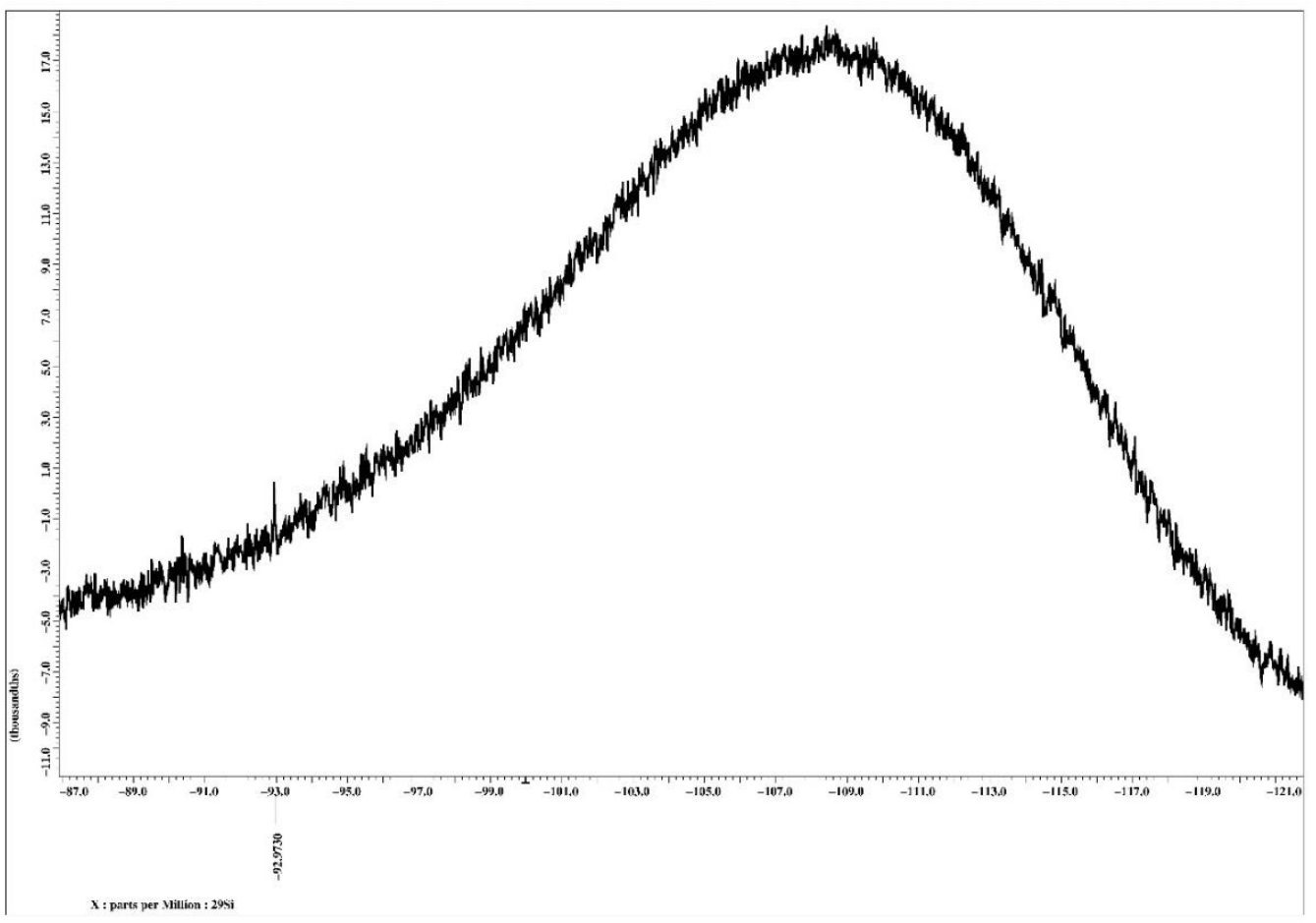

Figure S4. ${ }^{29} \mathrm{Si}$ NMR spectrum of compound 3 (in $\mathrm{C}_{6} \mathrm{D}_{6}$ ). 


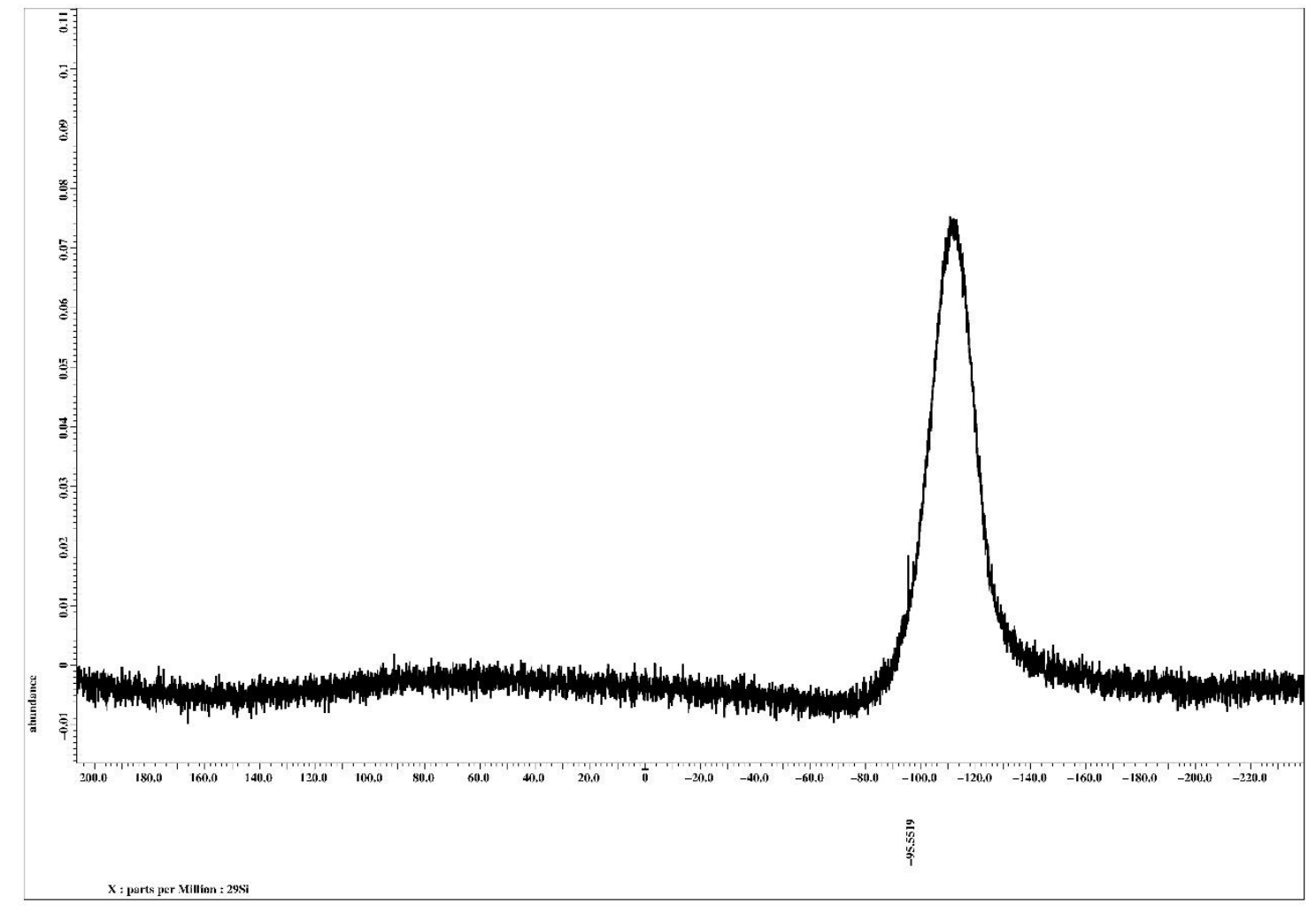

(a)

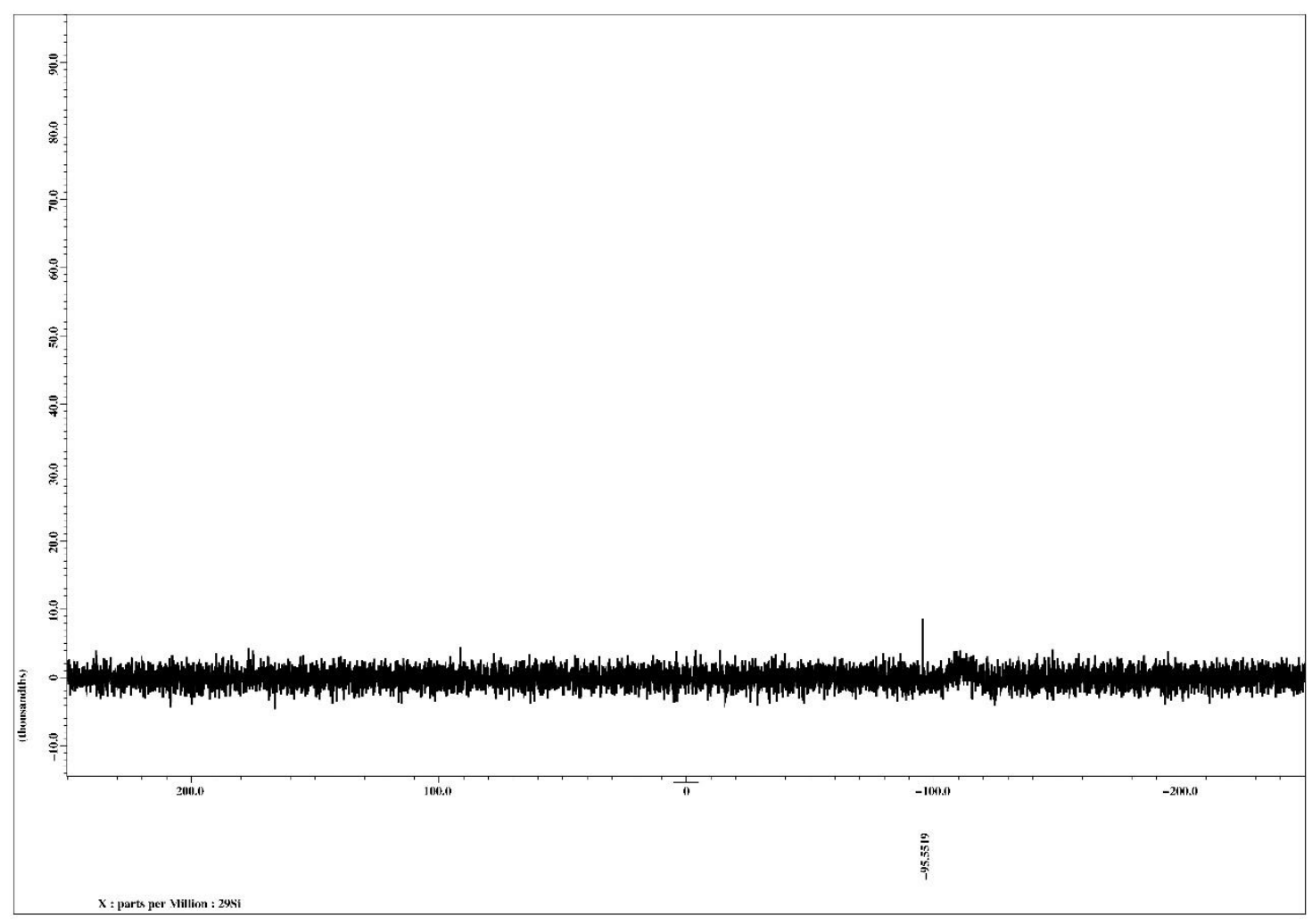

(b)

Page S27 


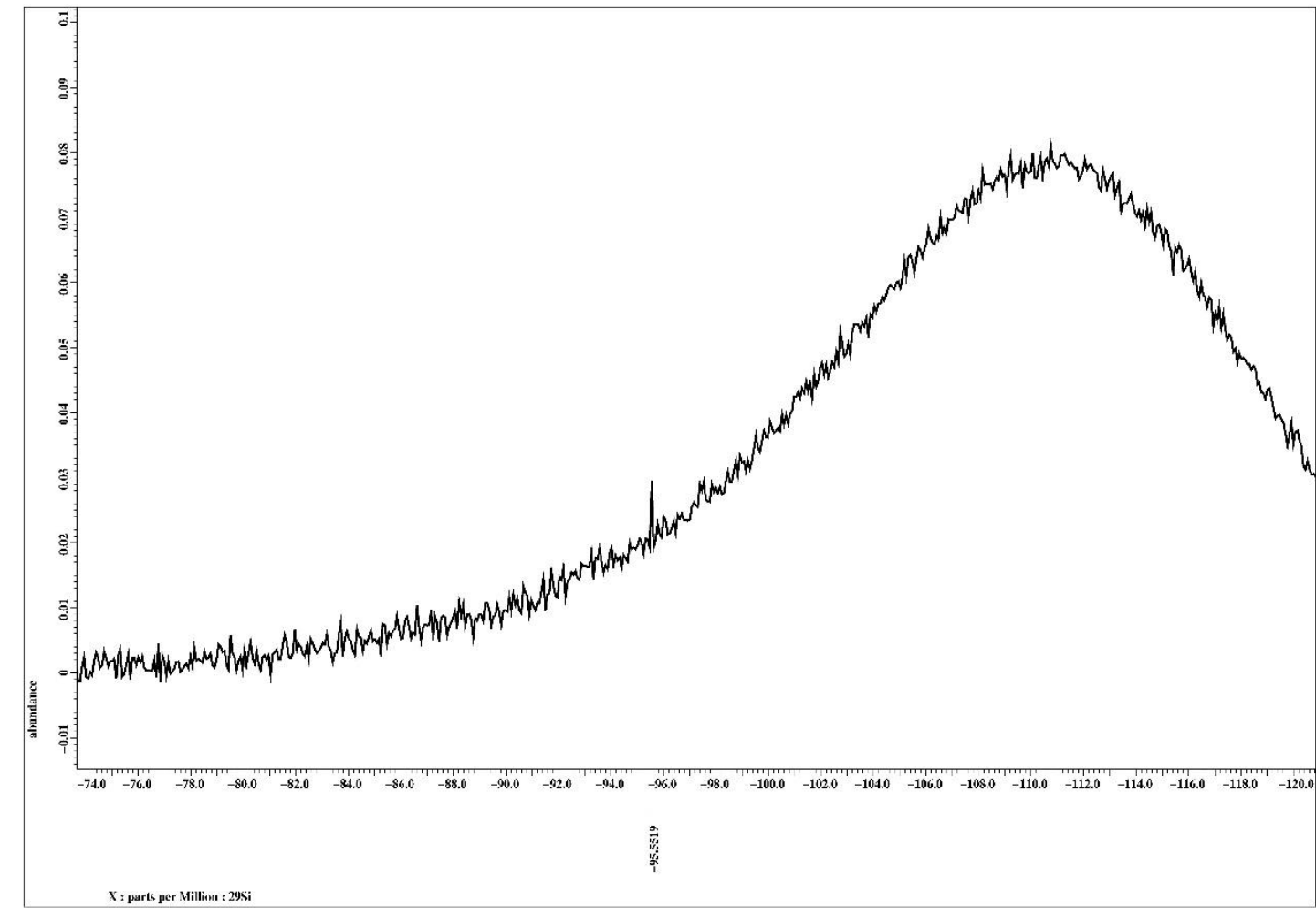

(c)

Figure S5. ${ }^{29} \mathrm{Si}\left\{{ }^{1} \mathrm{H}\right\}$ NMR spectrum of compound 3 (in $\mathrm{C}_{6} \mathrm{D}_{6}$ ), (a) before flattening the baseline, (b) after flattening the baseline, (c) focus view.

Single Mass Analysis

Tolerance $=10.0 \mathrm{PPM}, \mathrm{DBE}: \min =-1.5, \max =50.0$

Flerance $=10.0 \mathrm{PPM} /$

Number of isotope peaks used for $\mathrm{i}-\mathrm{FIT}=3$

Monoisotopic Mass, Even Electron lons
1 formula(e) evaluated with 1 results within limits (up to 50 closest results for each mass)

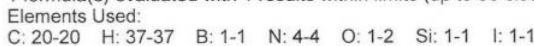

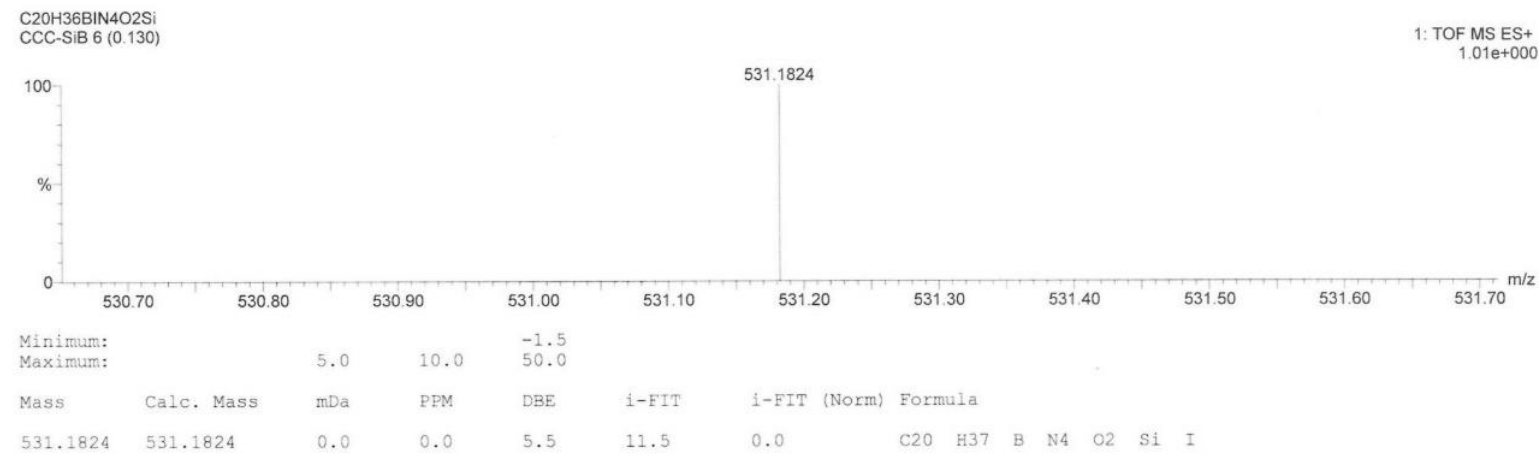

Figure S6. ESI-MS of 3. 


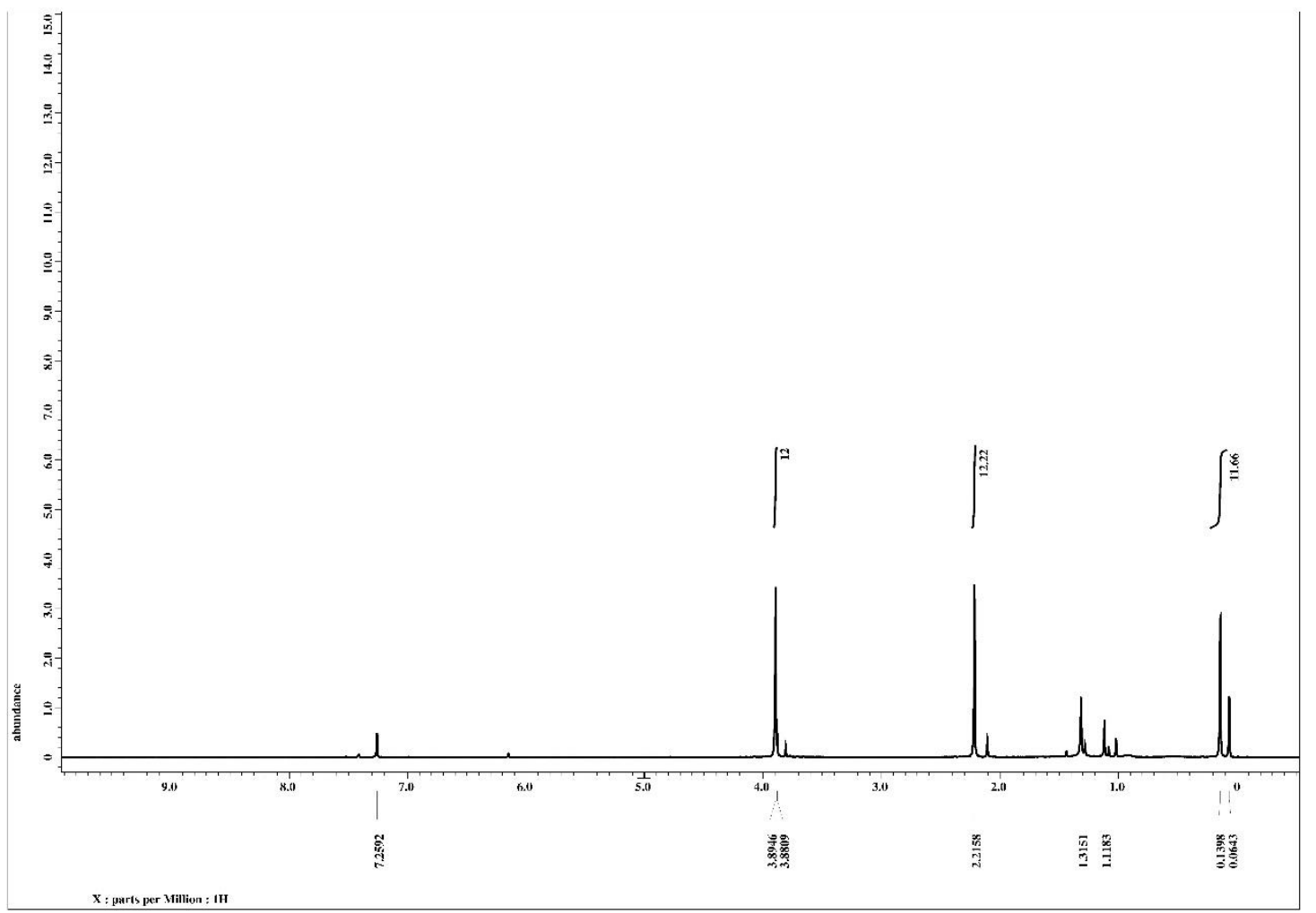

Figure S7. ${ }^{1} \mathrm{H}$ NMR spectrum of compound [( $\mathbf{I M e}_{\mathbf{M e}} \mathbf{B}$ pin]I (in $\left.\mathrm{CDCl}_{3}\right)$.

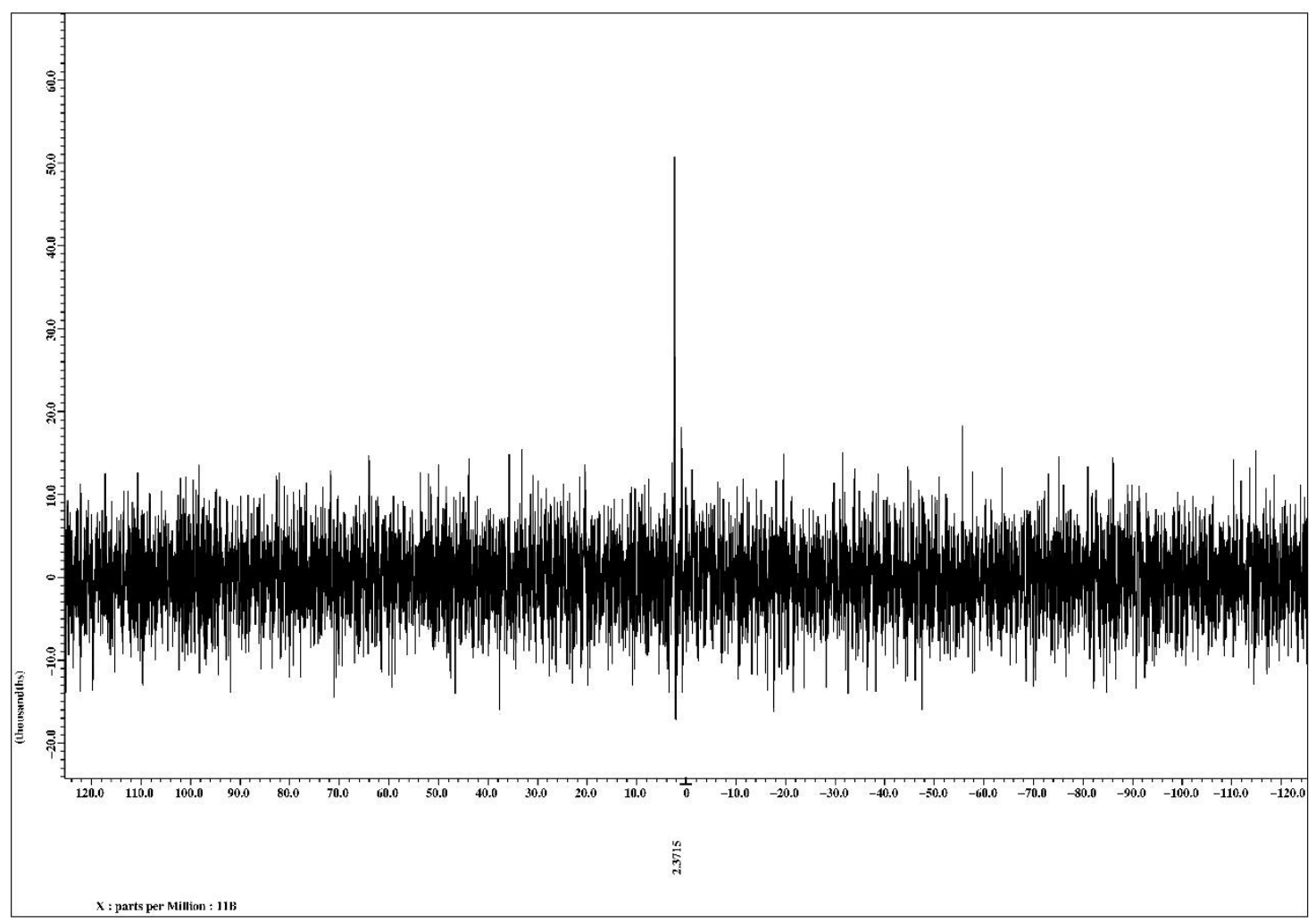

Figure S8. ${ }^{11} \mathrm{~B}$ NMR spectrum of compound [( $\left.\left.\mathbf{I}_{\mathbf{M e}}\right)_{2} \mathbf{B p i n}\right] \mathbf{I}$ (in $\mathrm{CDCl}_{3}$ ). 


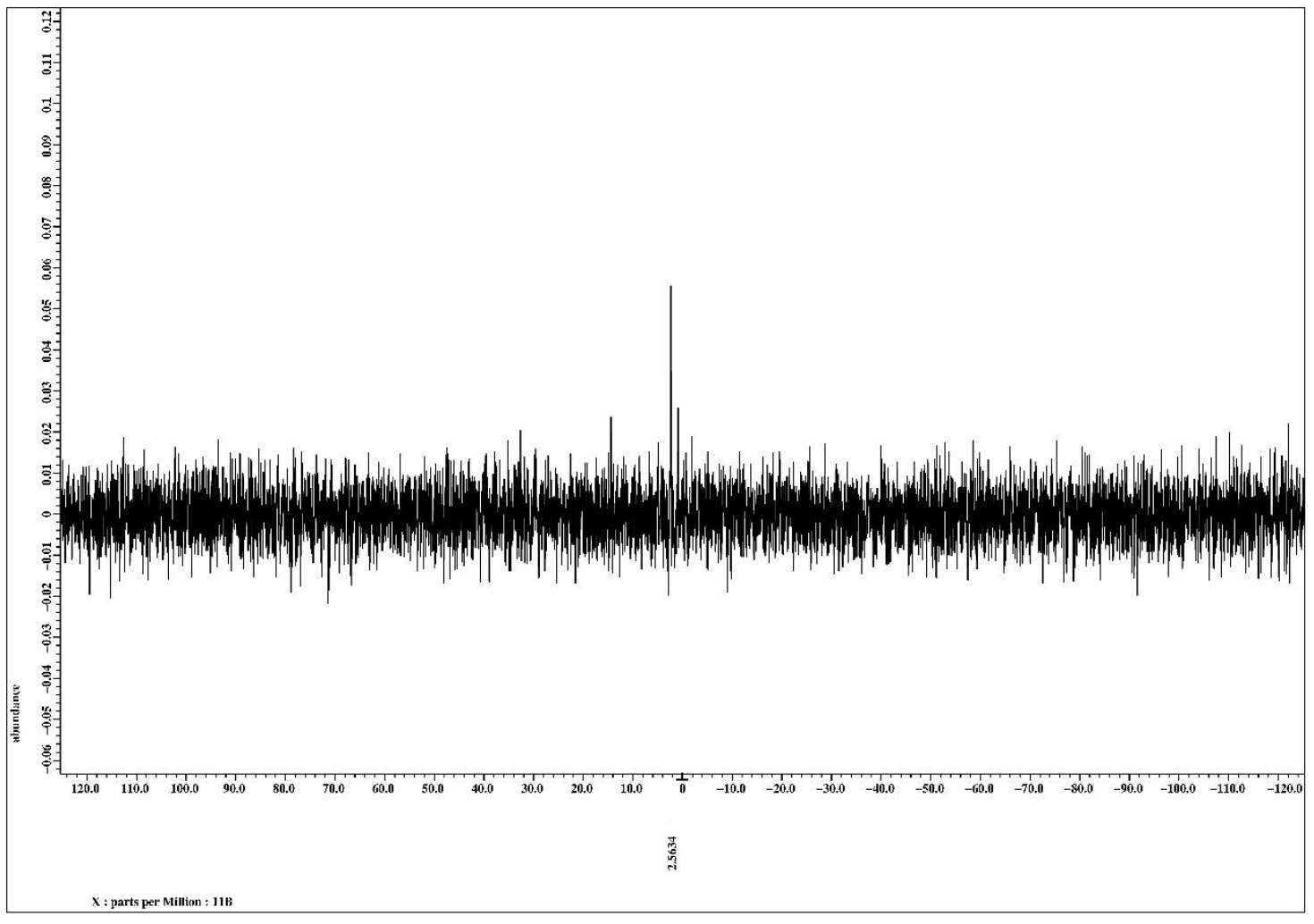

Figure S9. ${ }^{11} \mathrm{~B}\left\{{ }^{1} \mathrm{H}\right\}$ NMR spectrum of compound [( $\left.\left.\mathbf{I}_{\mathbf{M e}}\right)_{2} \mathbf{B p i n}\right] \mathbf{I}\left(\right.$ in $\left.\mathrm{CDCl}_{3}\right)$.

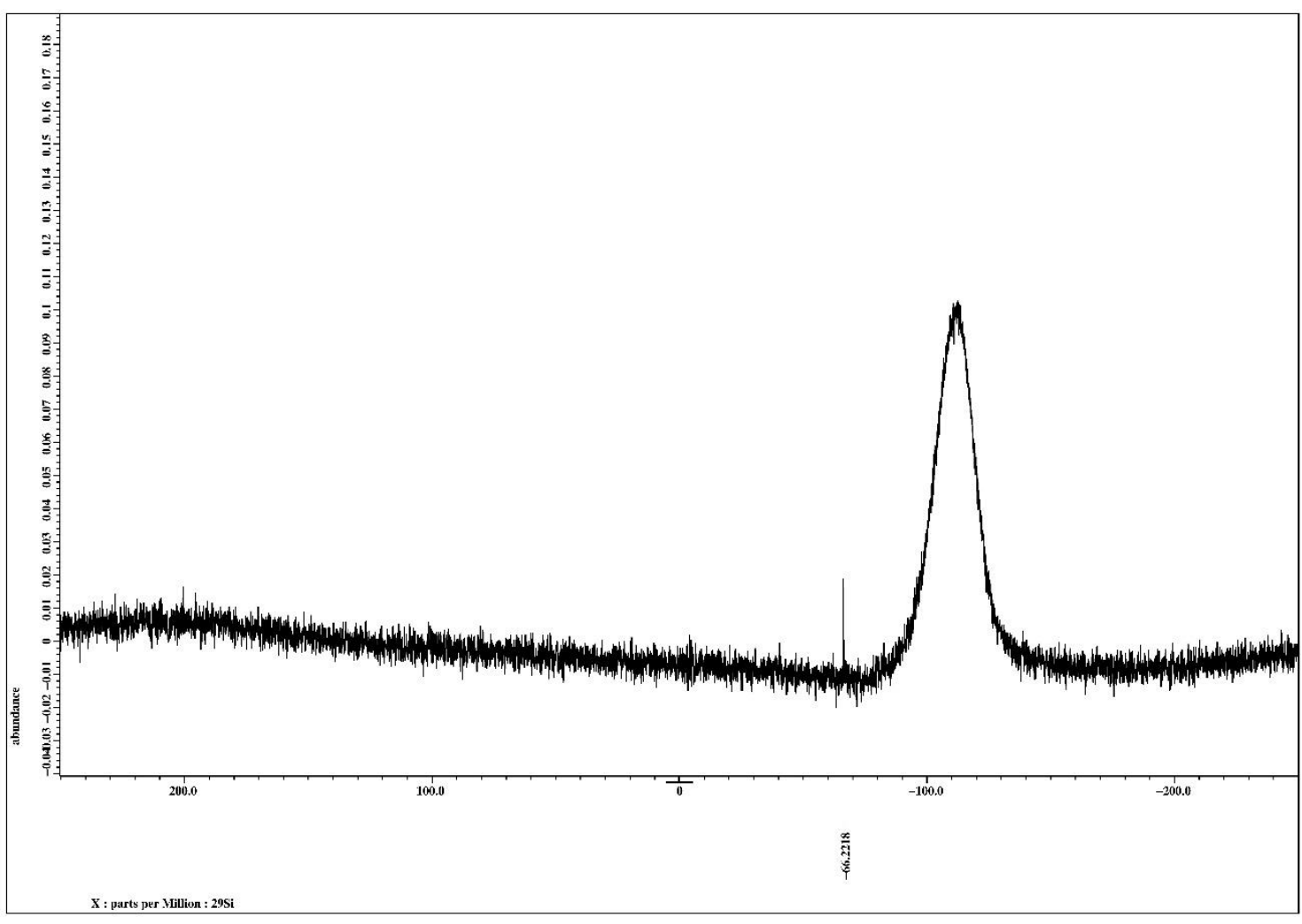

Figure S10. ${ }^{29} \mathrm{Si}\left\{{ }^{1} \mathrm{H}\right\}$ NMR spectrum of compound Int01 (in $d_{5}$-pyridine). 

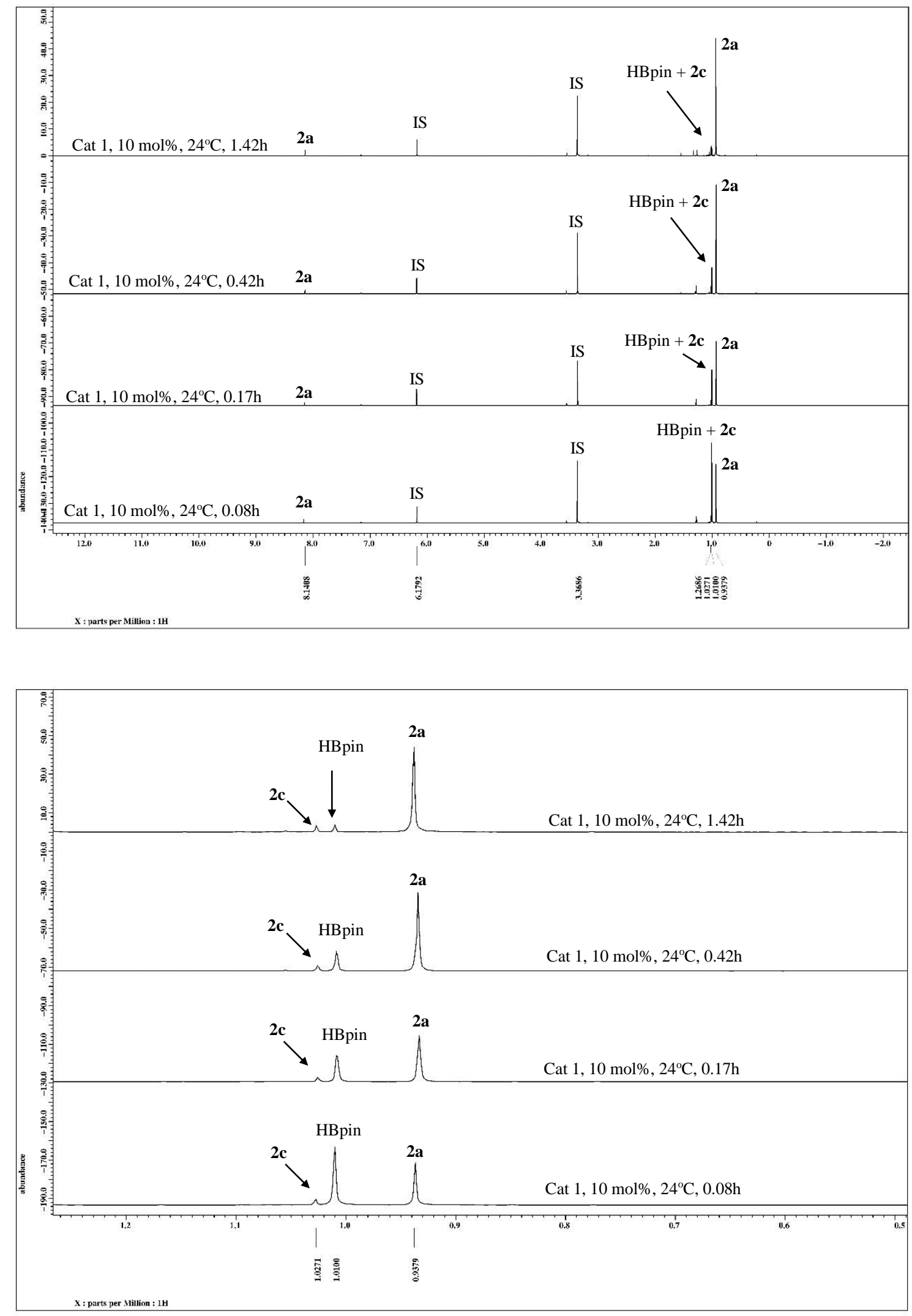

(a)

Page S31 

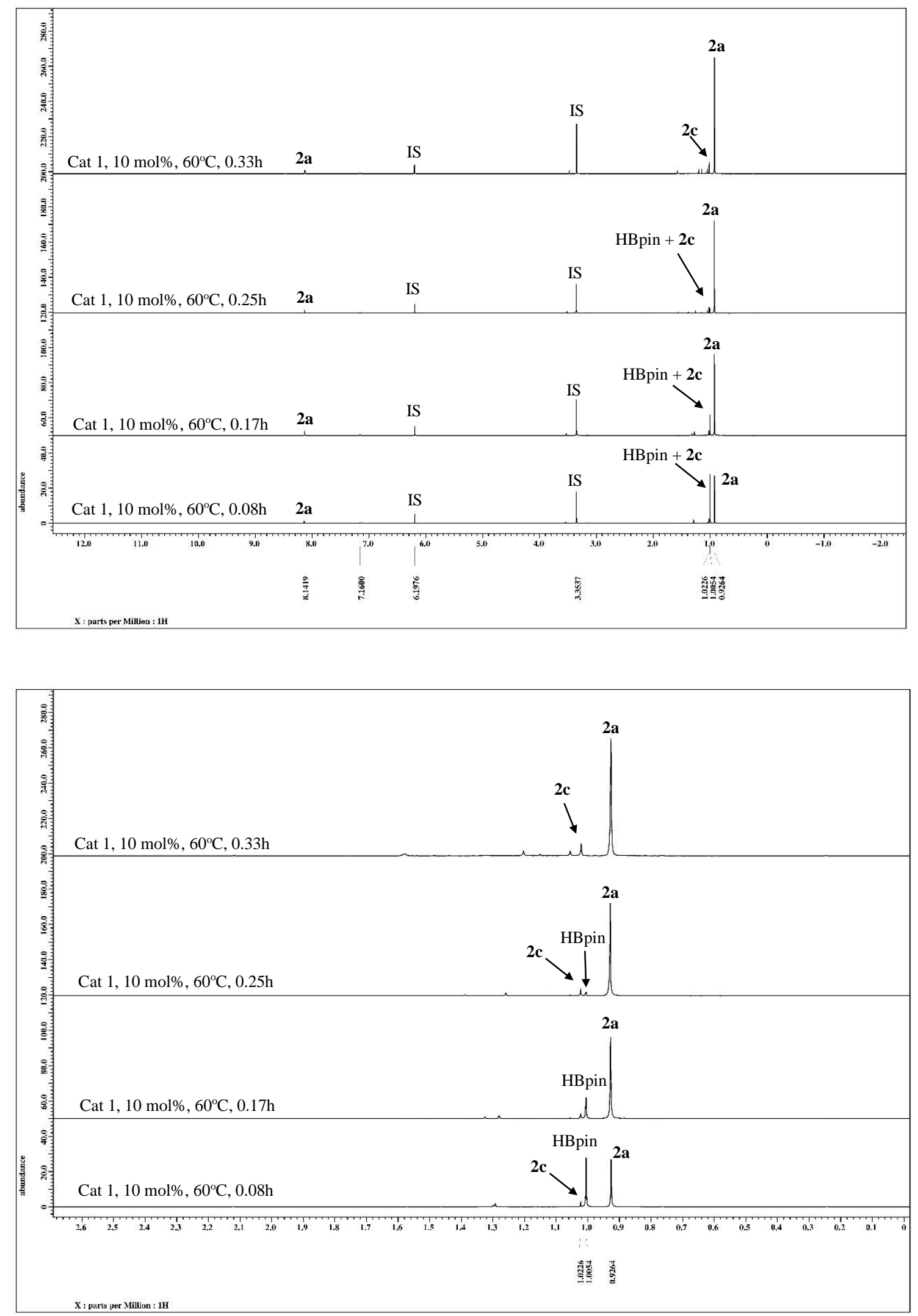

(b)

Page S32 

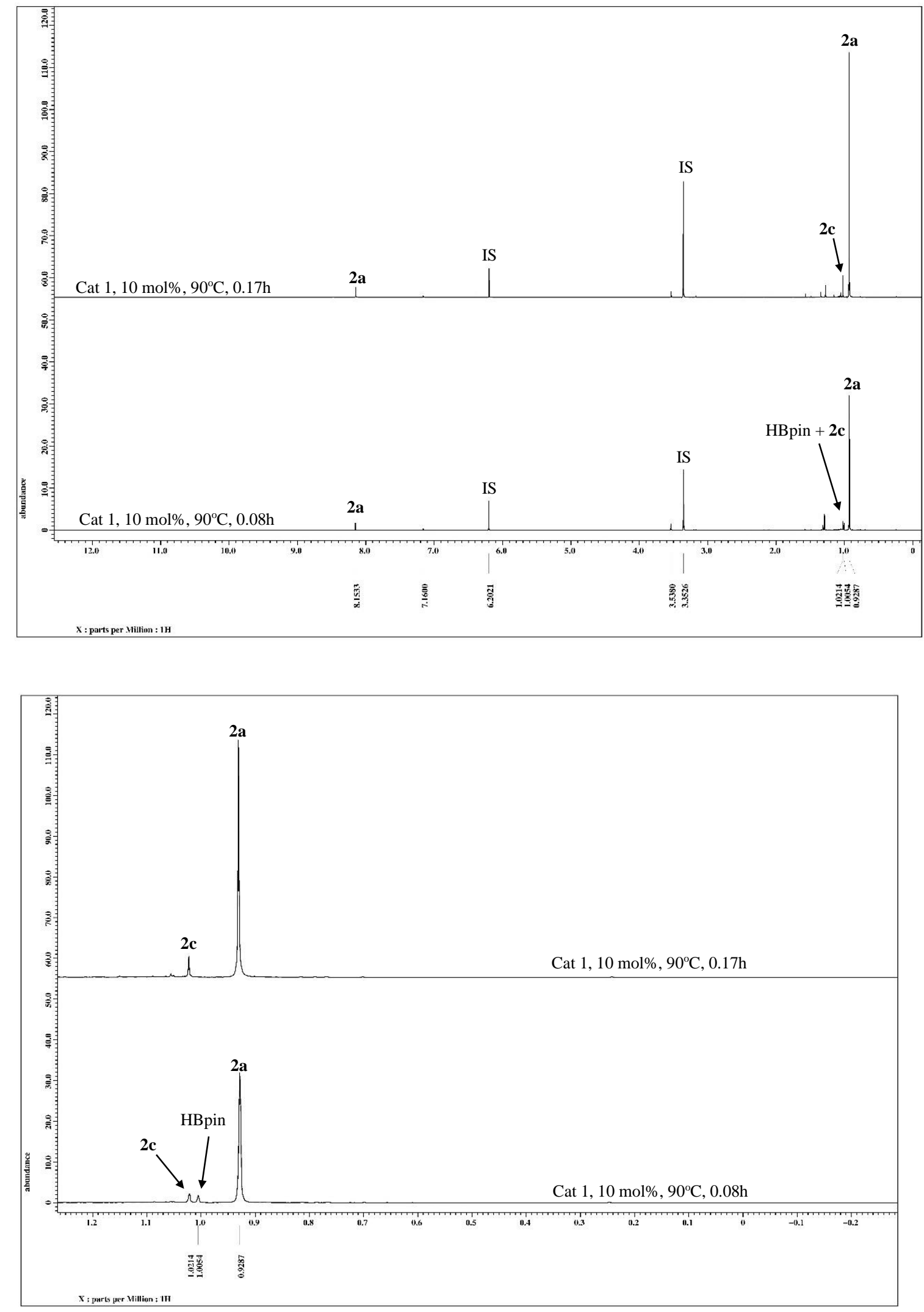

(c)

Figure S11. ${ }^{1} \mathrm{H}$ NMR spectra of the reaction of $\mathrm{CO}_{2}$ with $\mathrm{HBpin}$ catalysed by $\mathbf{1}(10 \mathrm{~mol} \%$, in $\mathrm{C}_{6} \mathrm{D}_{6}$ ), at (a) $24^{\circ} \mathrm{C}$, (b) $60^{\circ} \mathrm{C}$ and (c) $90^{\circ} \mathrm{C}$. 


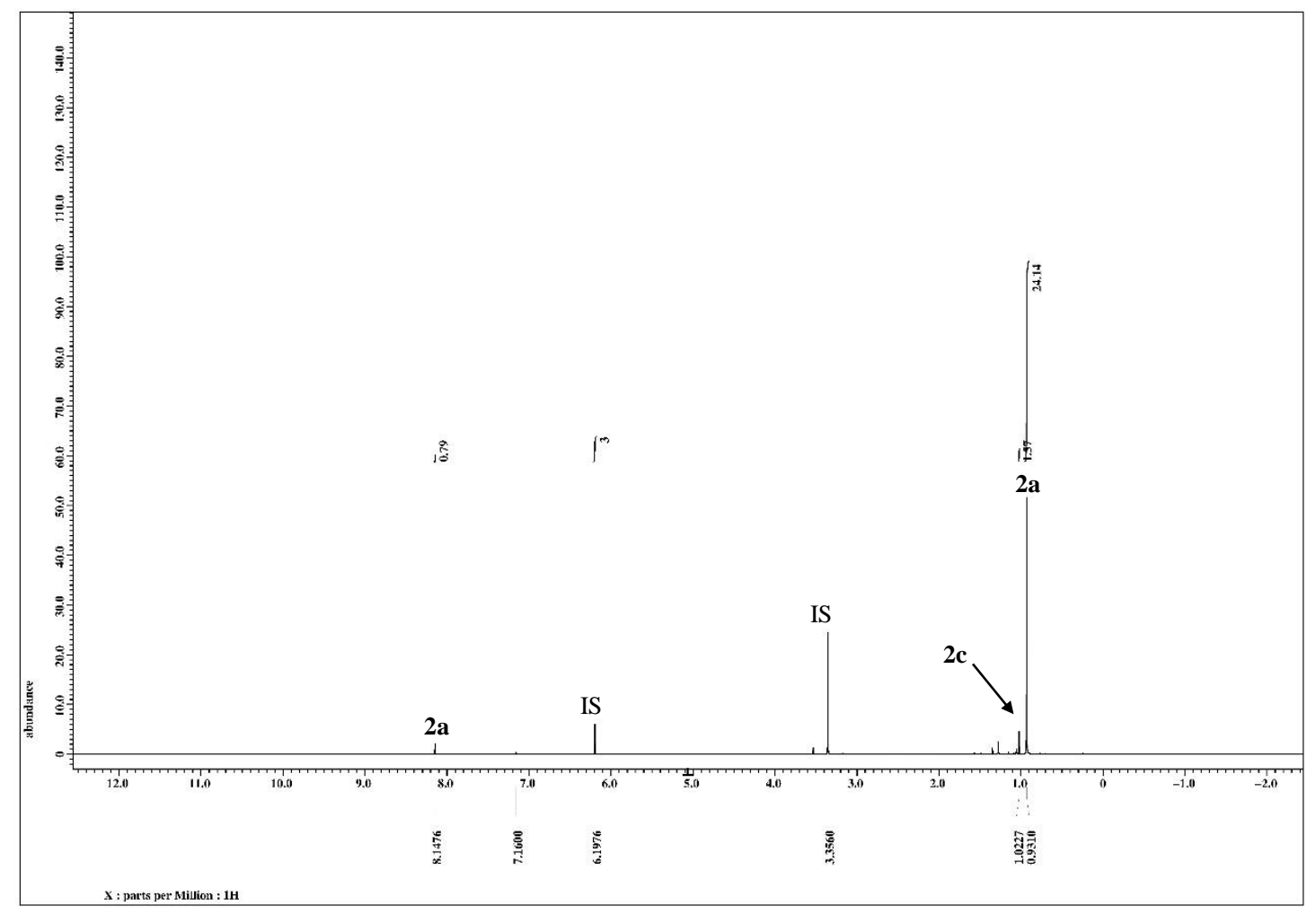

(a)

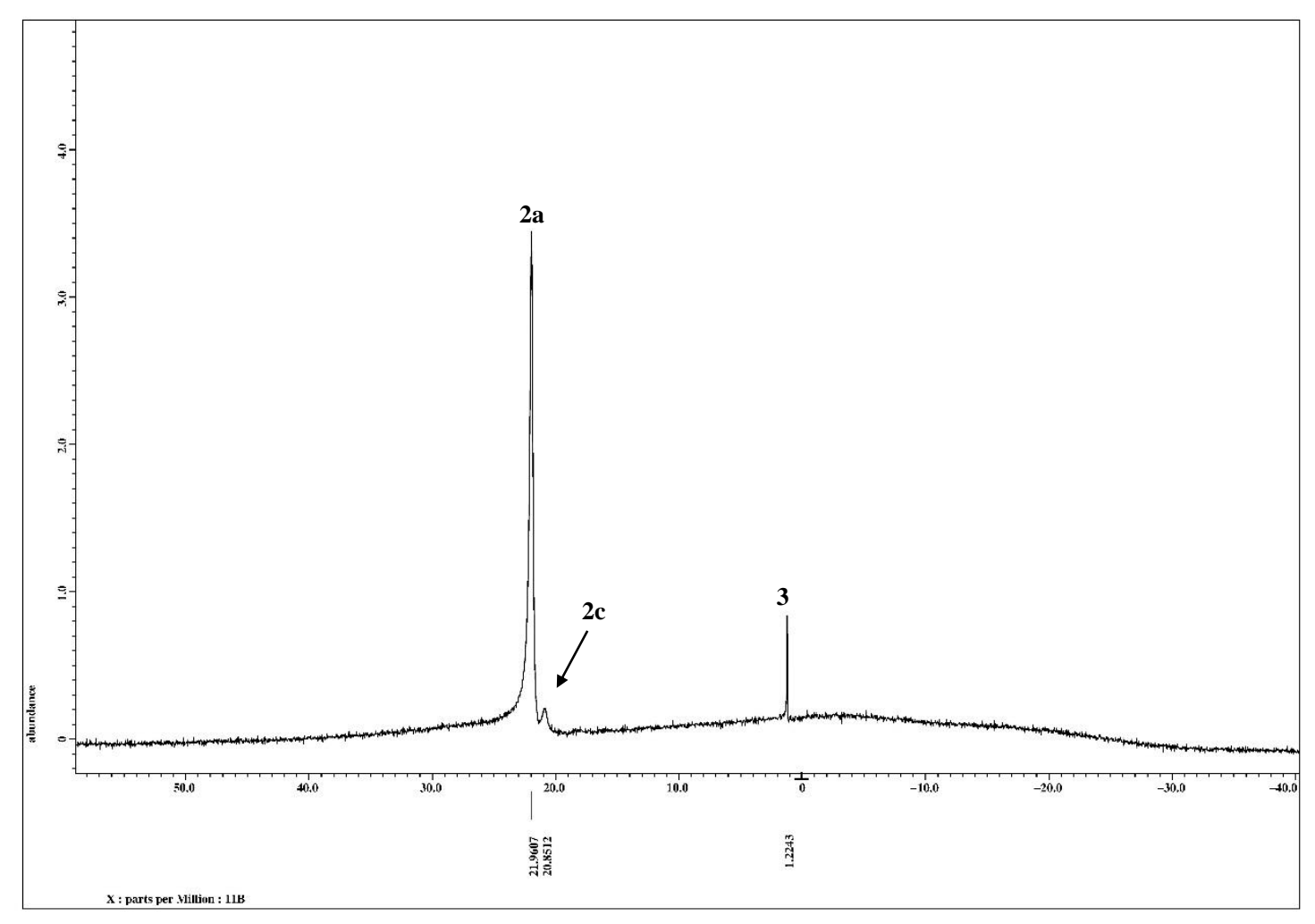

(b)

Page S34 


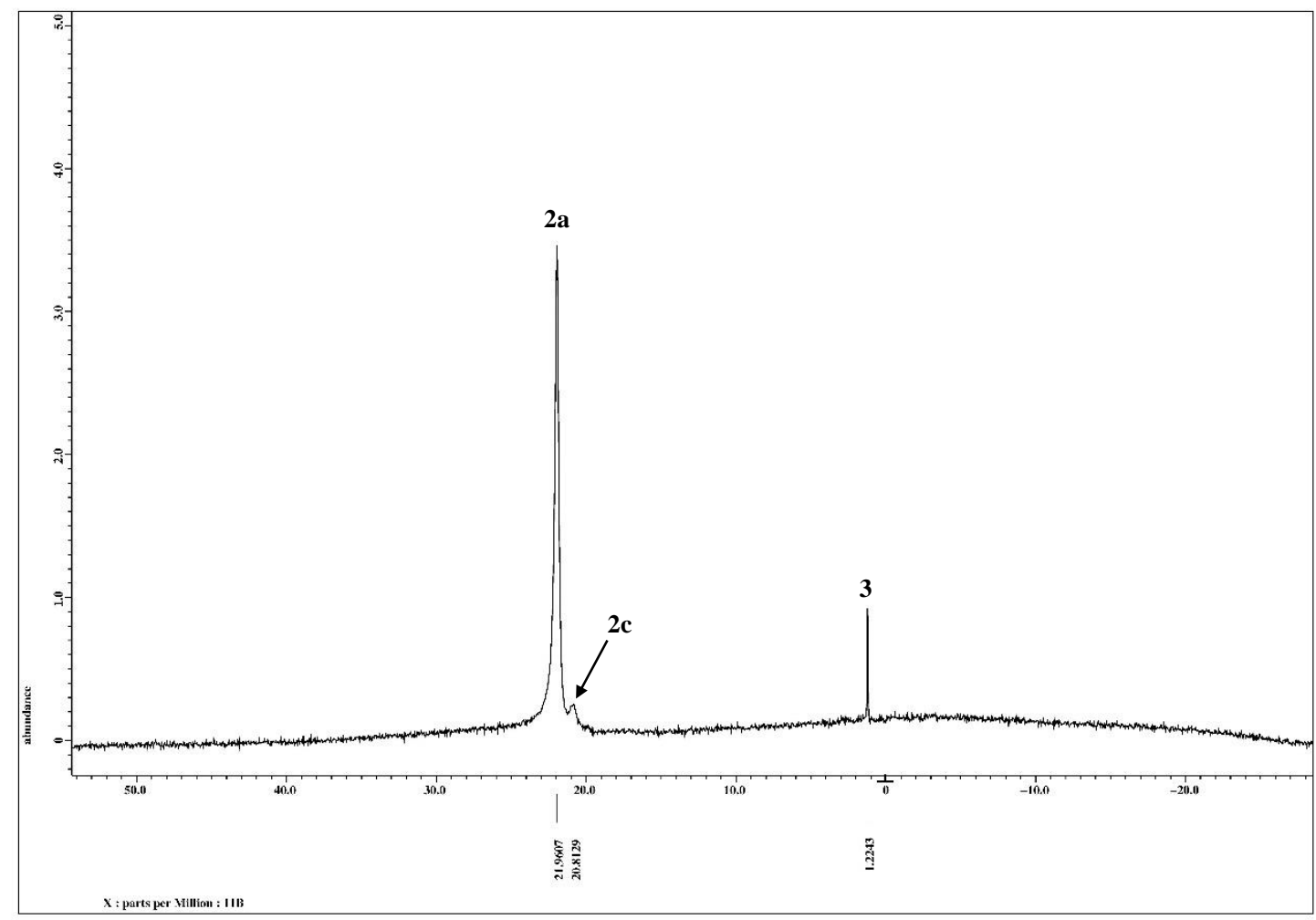

(c)

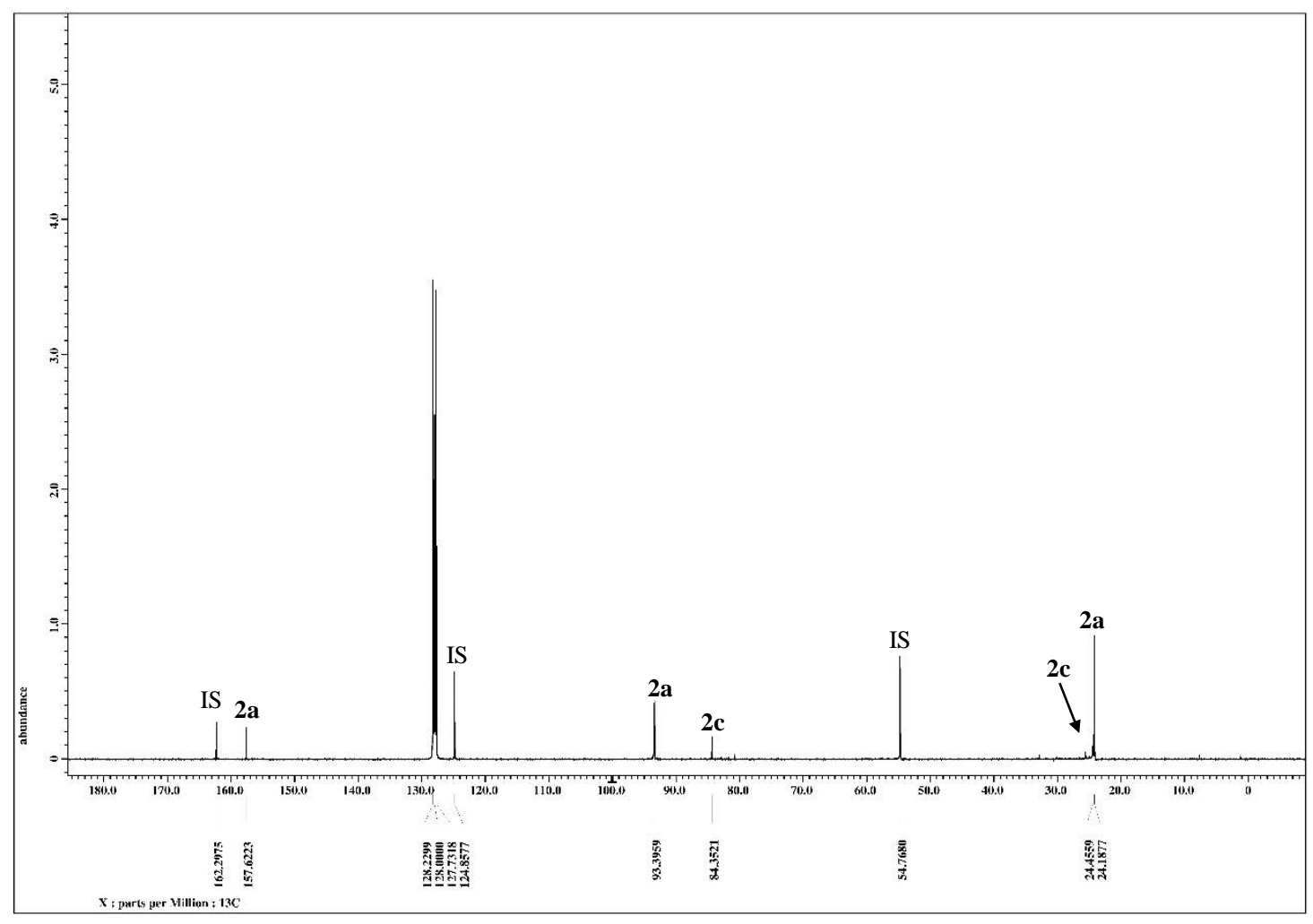

(d)

Figure S12. NMR spectra of the resultant reaction of $\mathrm{CO}_{2}$ with $\mathrm{HB}$ pin catalysed by $\mathbf{1}$ (10 mol $\%$, in $\mathrm{C}_{6} \mathrm{D}_{6}$ ), (a) ${ }^{1} \mathrm{H}$, (b) ${ }^{11} \mathrm{~B}$, (c) ${ }^{11} \mathrm{~B}\left\{{ }^{1} \mathrm{H}\right\}$ and (d) ${ }^{13} \mathrm{C}\left\{{ }^{1} \mathrm{H}\right\}$. 

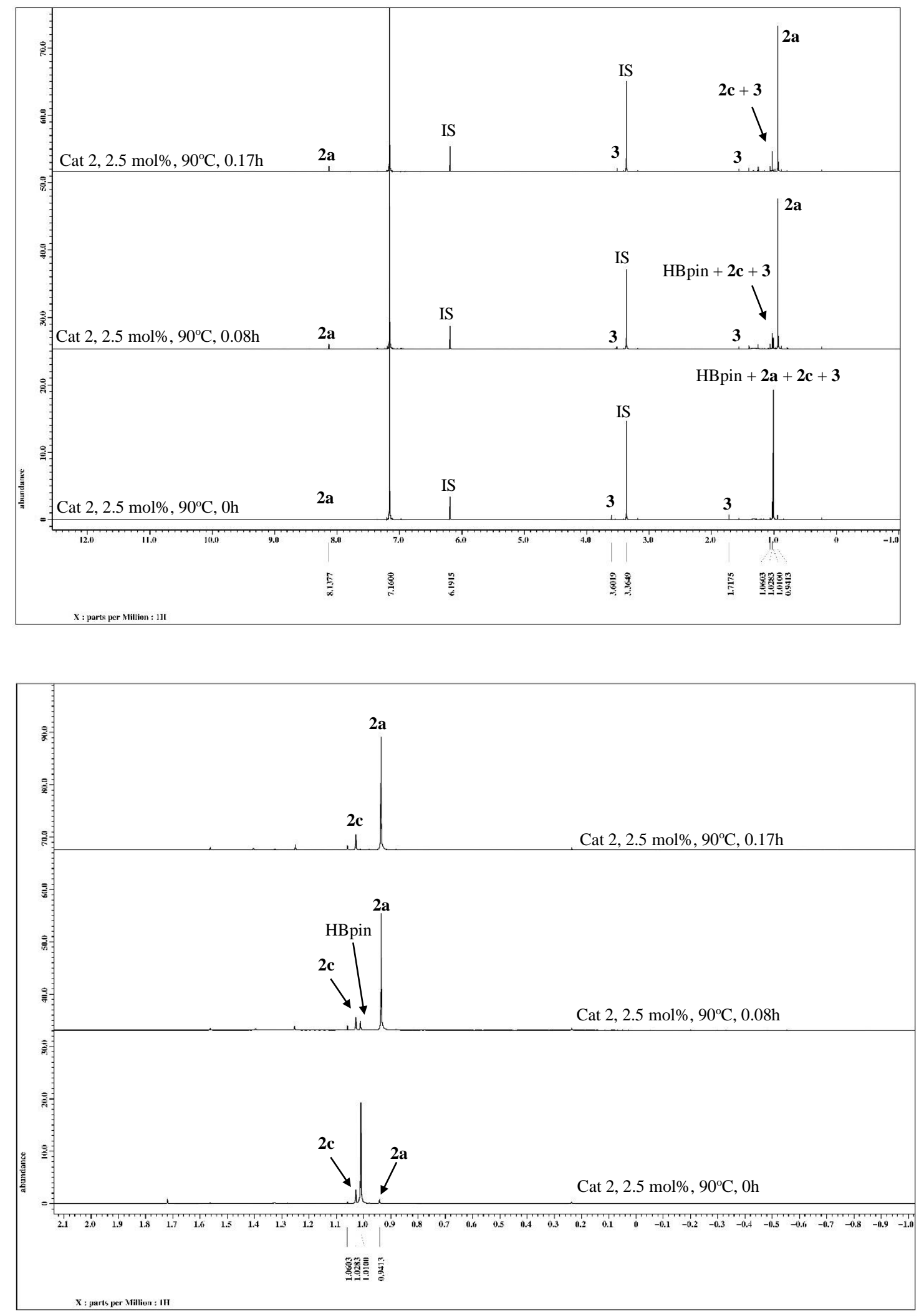

(a)

Page S36 


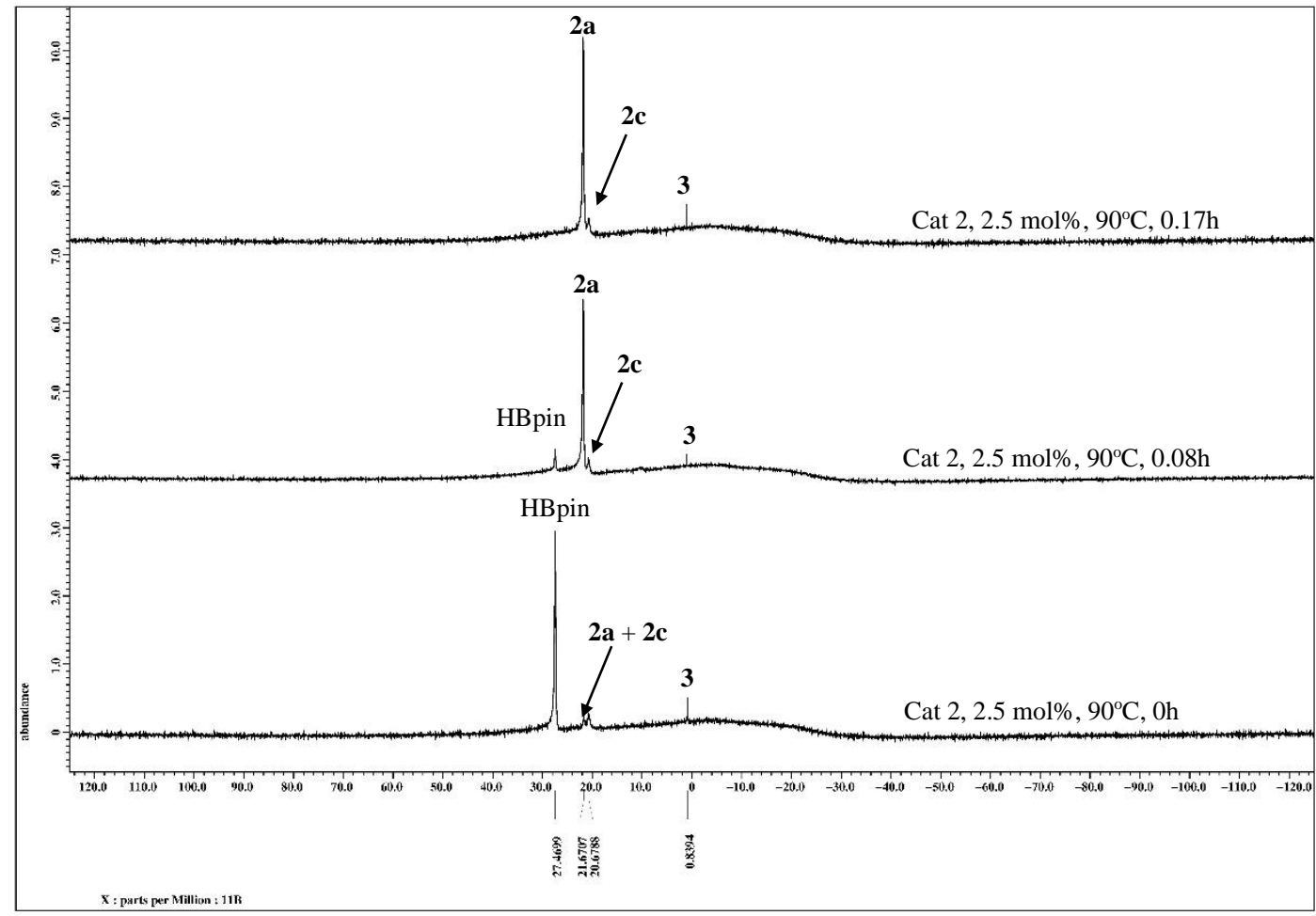

(b)

Figure S13. NMR spectra of the reaction of $\mathrm{CO}_{2}$ with $\mathrm{HBpin}$ catalysed by $\mathbf{3}\left(2.5 \mathrm{~mol} \%, 90^{\circ} \mathrm{C}\right.$, in $\mathrm{C}_{6} \mathrm{D}_{6}$ ), (a) ${ }^{1} \mathrm{H}$ and (b) ${ }^{11} \mathrm{~B}\left\{{ }^{1} \mathrm{H}\right\}$. 


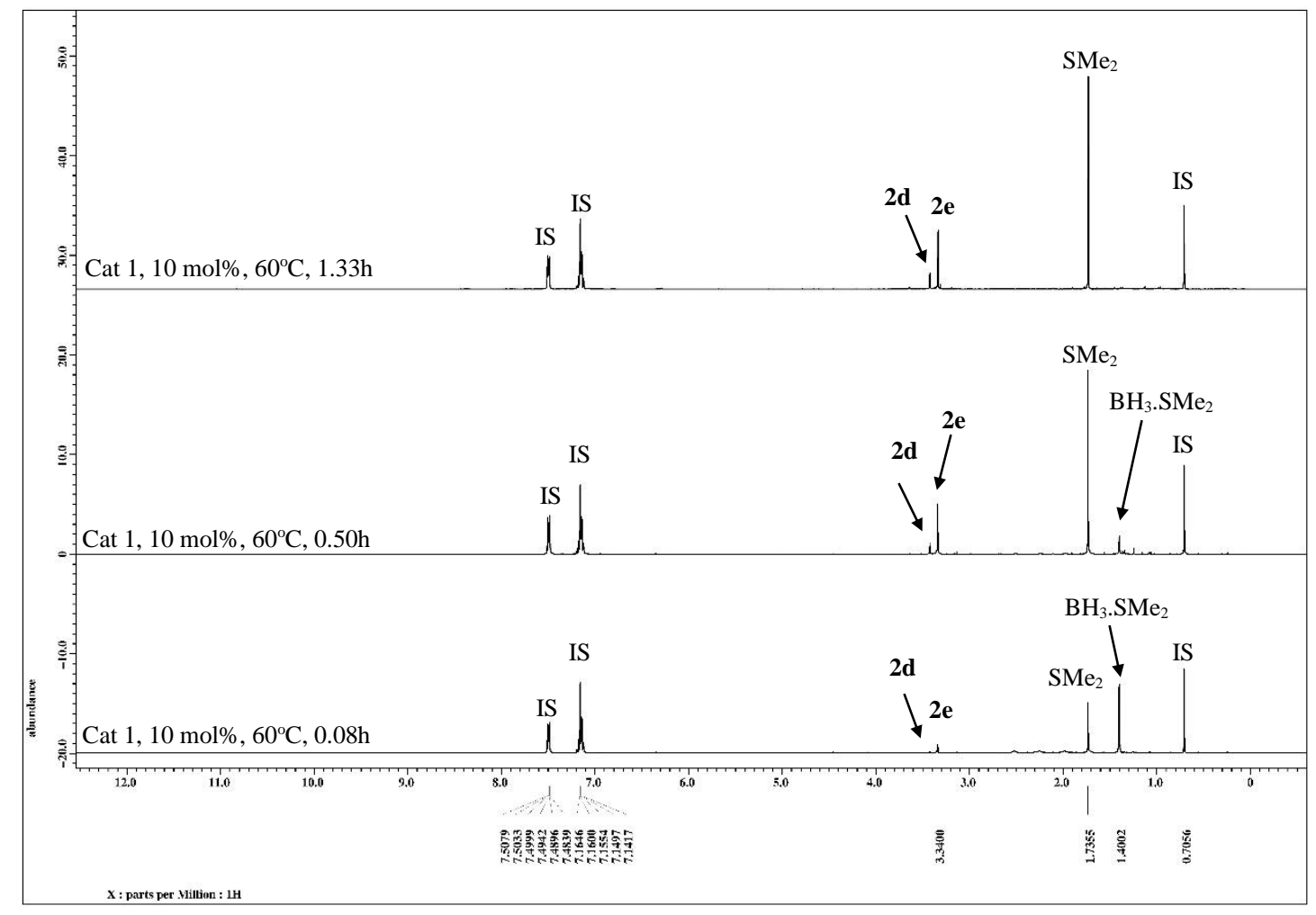

(a)

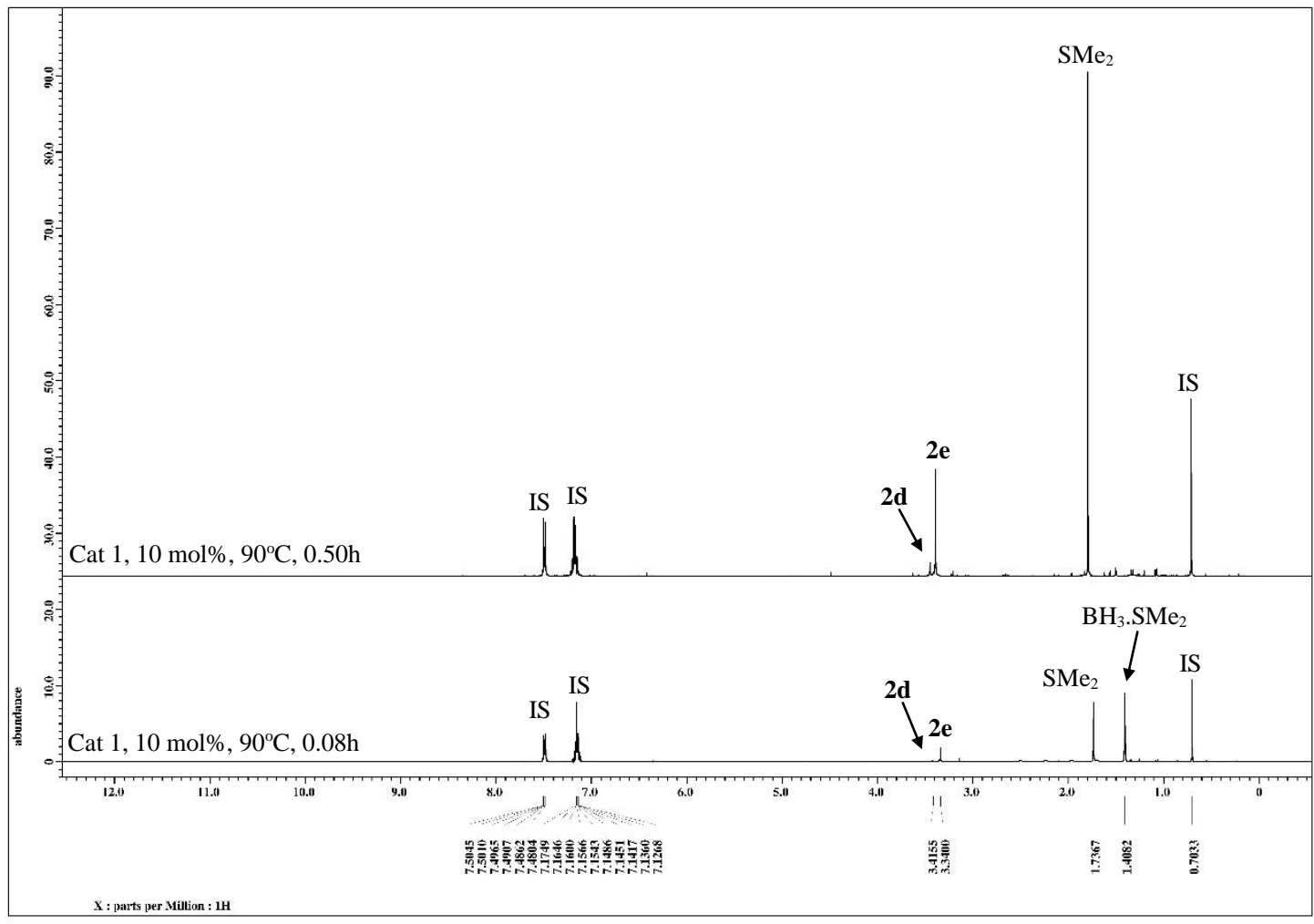

(b)

Figure S14. ${ }^{1} \mathrm{H}$ NMR of the reaction of $\mathrm{CO}_{2}$ with $\mathrm{BH}_{3} \cdot \mathrm{SMe}_{2}$ catalysed by 1 (10 mol \%, in $\mathrm{C}_{6} \mathrm{D}_{6}$ ), at (a) $60^{\circ} \mathrm{C}$ and (b) $90^{\circ} \mathrm{C}$. 


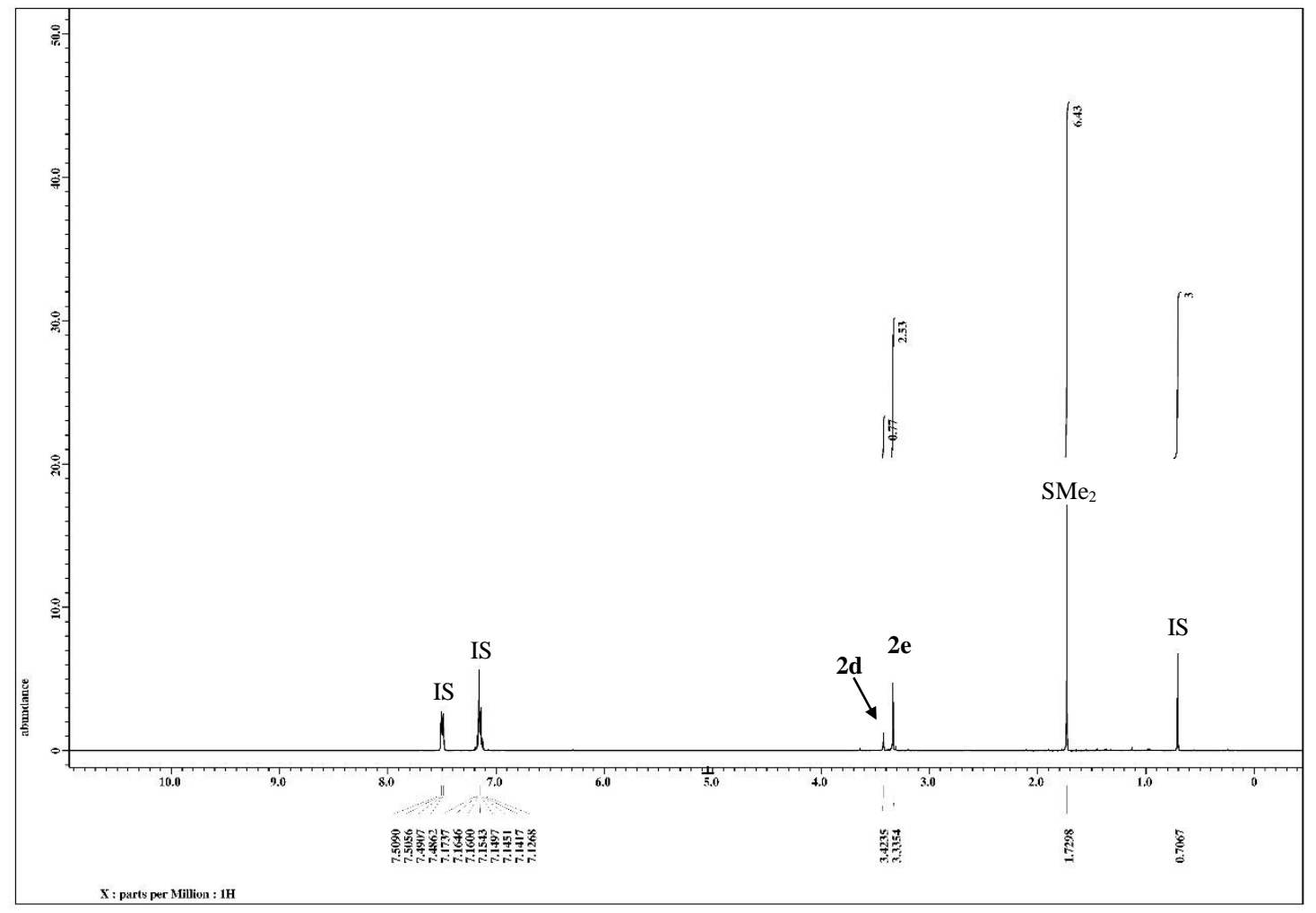

(a)

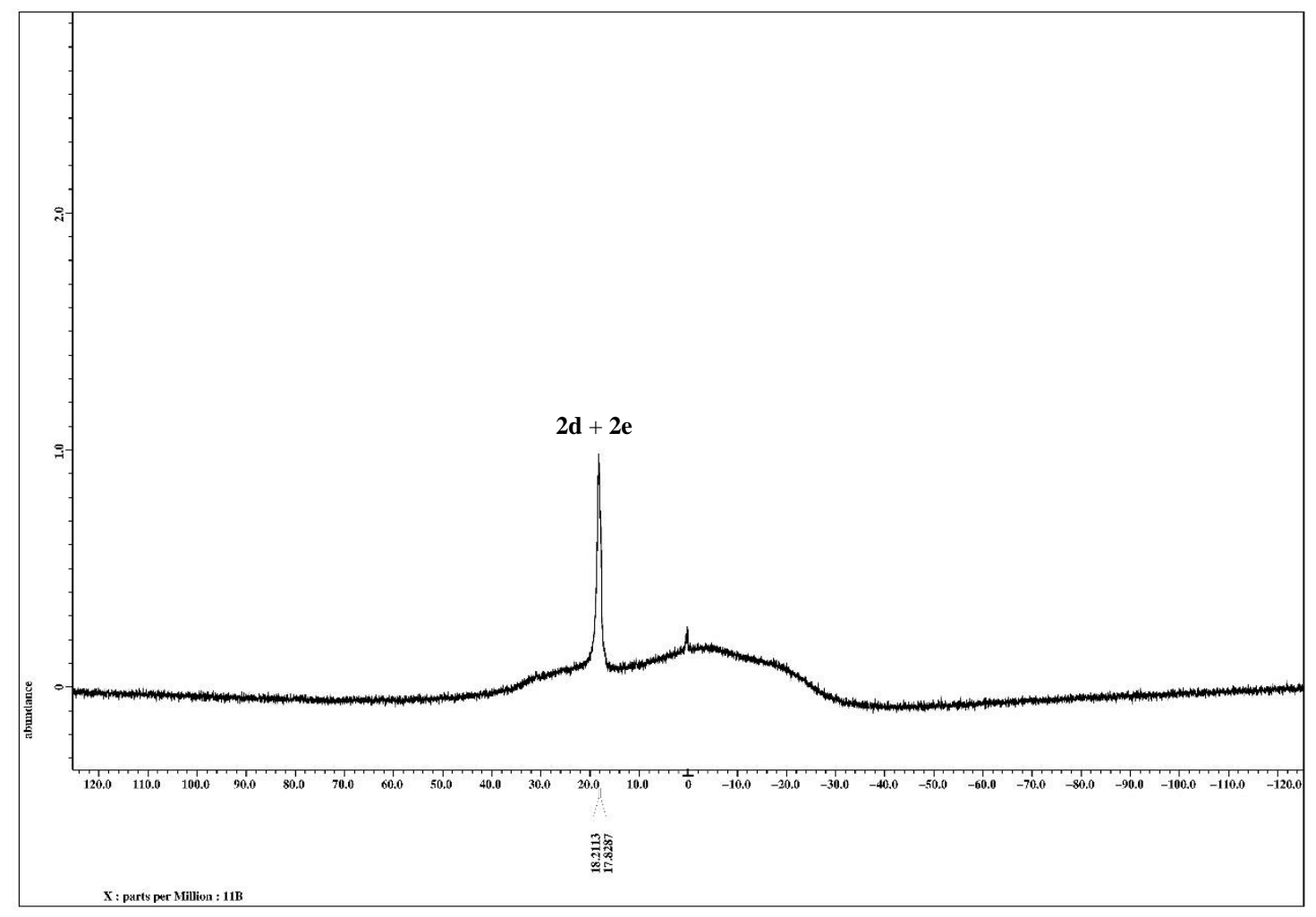

(b)

Page S39 


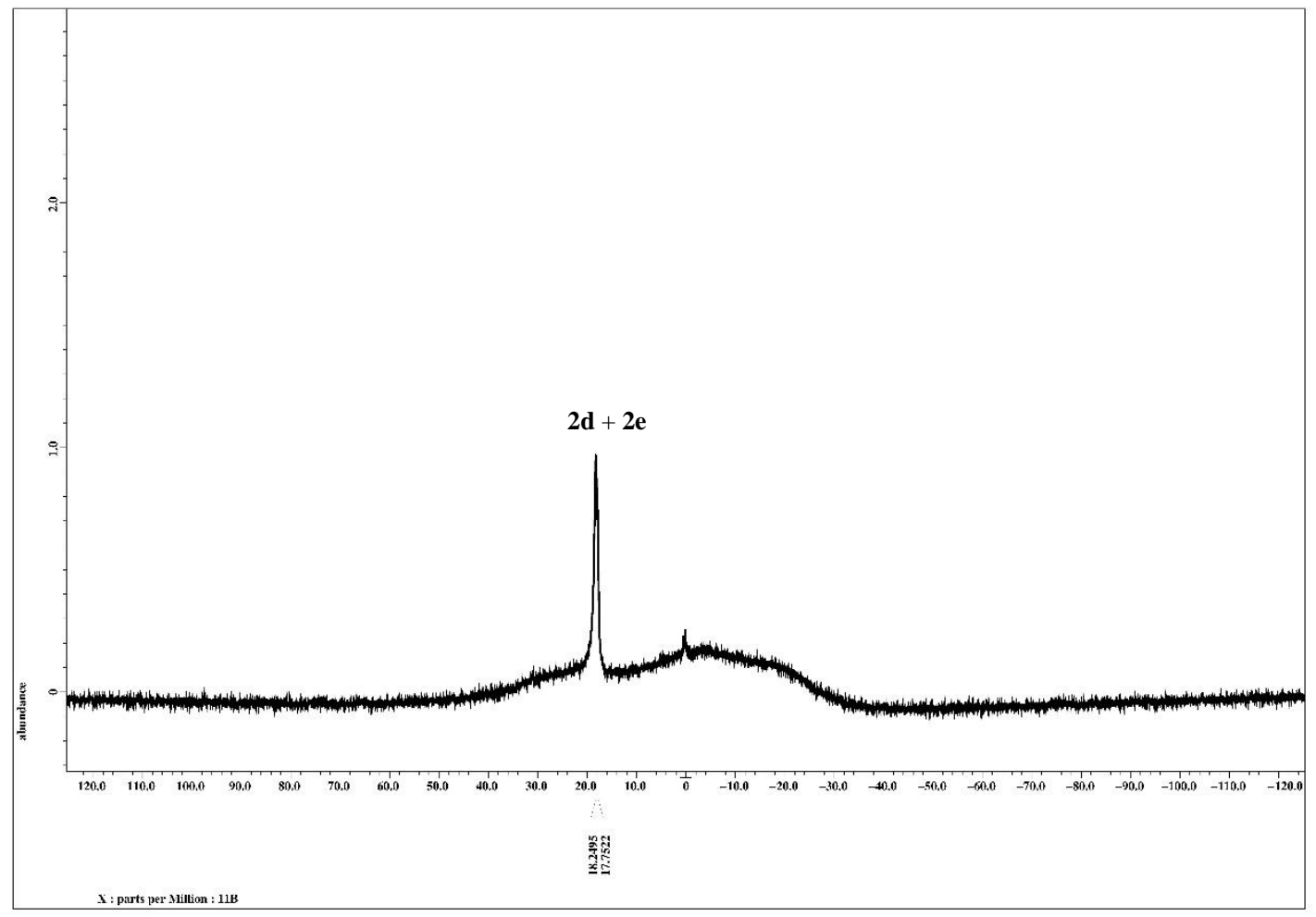

(c)

Figure S15. NMR spectra of the resultant reaction of $\mathrm{CO}_{2}$ with $\mathrm{BH}_{3} \cdot \mathrm{SMe}_{2}$ catalysed by 1 (10 mol \%, in $\mathrm{C}_{6} \mathrm{D}_{6}$ ), (a) ${ }^{1} \mathrm{H}$, (b) ${ }^{11} \mathrm{~B}$, (c) ${ }^{11} \mathrm{~B}\left\{{ }^{1} \mathrm{H}\right\}$. 


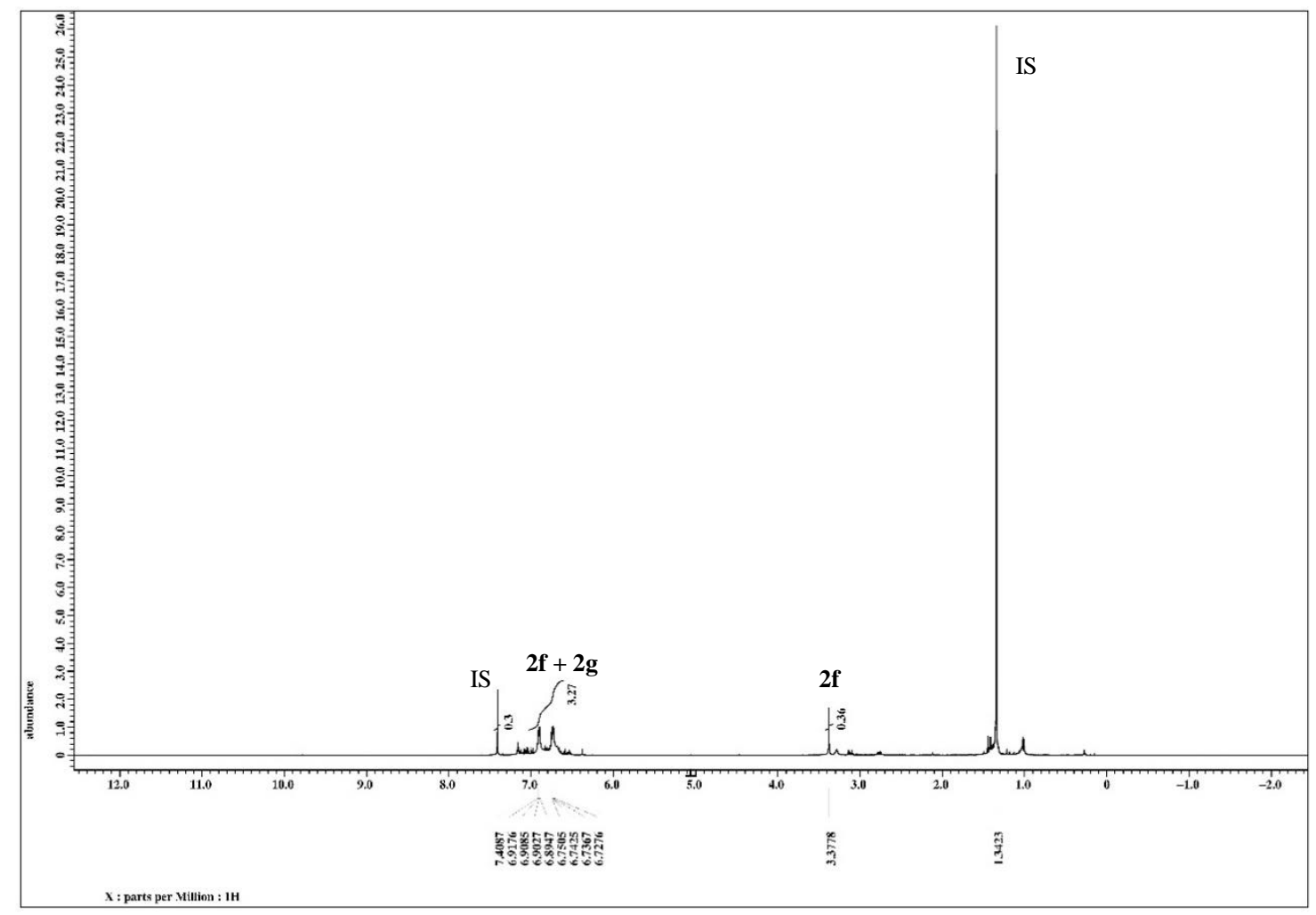

(a)

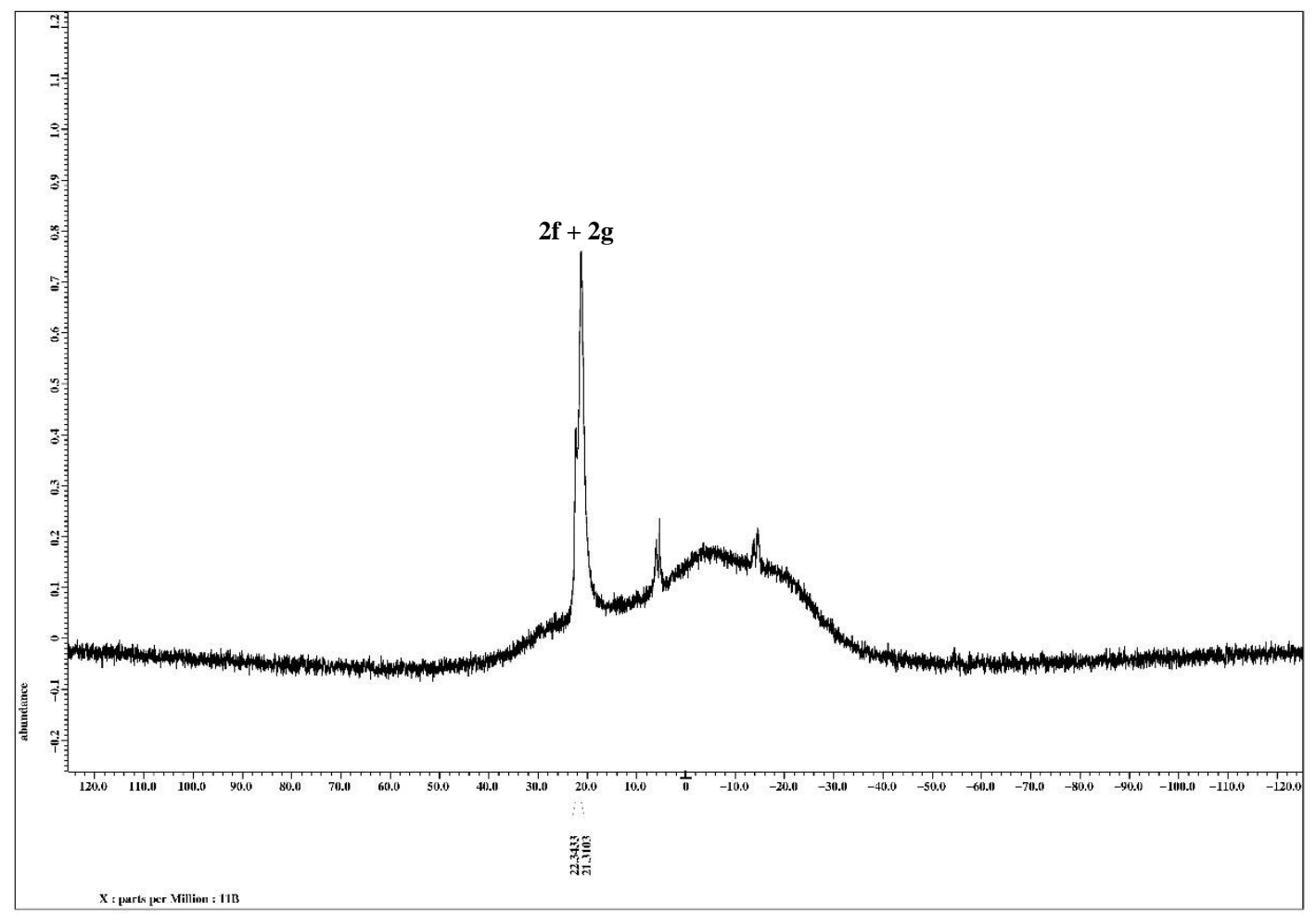

(b)

Page S41 


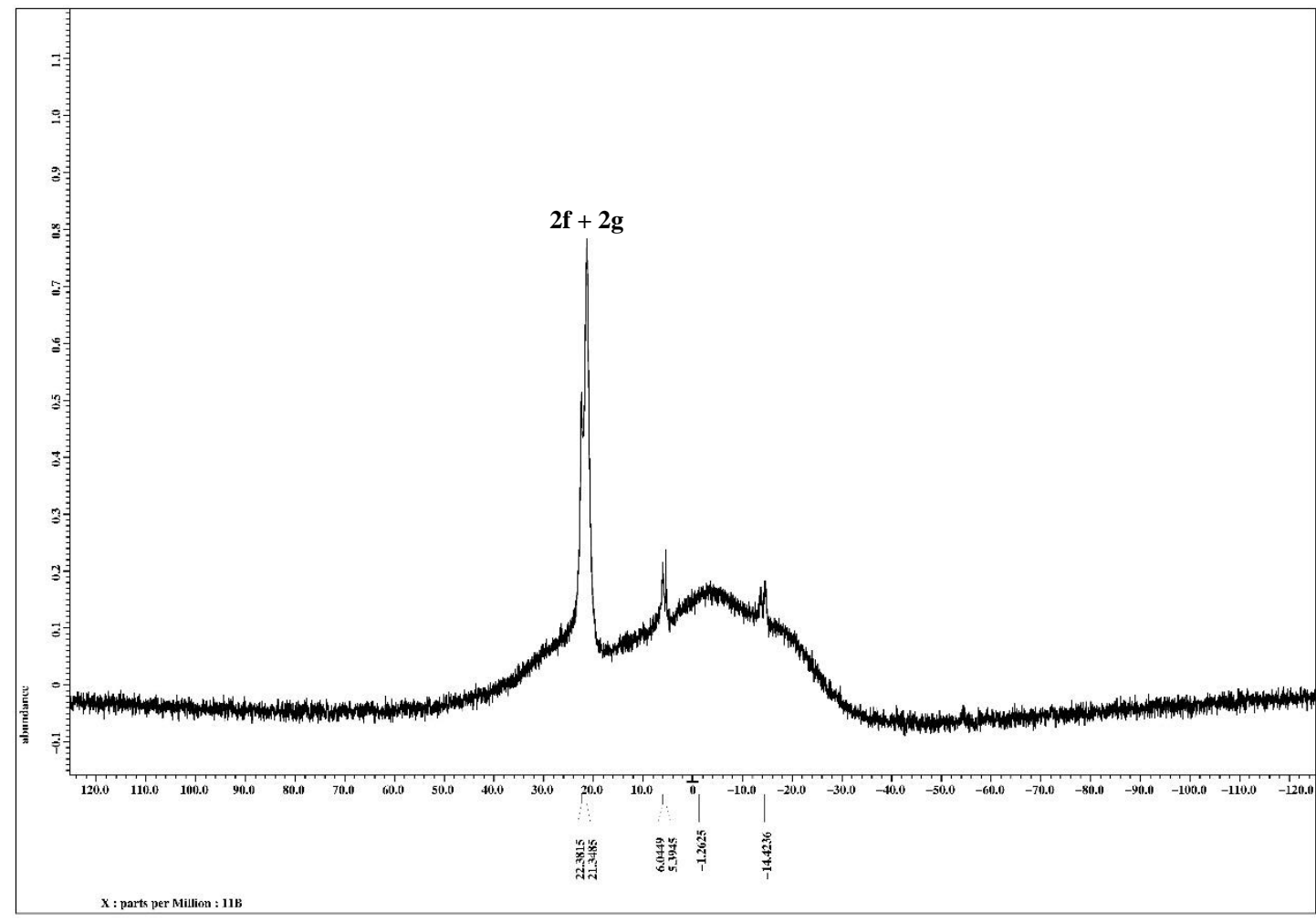

(c)

Figure S16. NMR spectra of the resultant reaction of $\mathrm{CO}_{2}$ with $\mathrm{HBcat}$ catalysed by $\mathbf{1}$ (10 mol $\%$, in $\mathrm{C}_{6} \mathrm{D}_{6}$ ), (a) ${ }^{1} \mathrm{H}$, (b) ${ }^{11} \mathrm{~B}$, (c) ${ }^{11} \mathrm{~B}\left\{{ }^{1} \mathrm{H}\right\}$. 


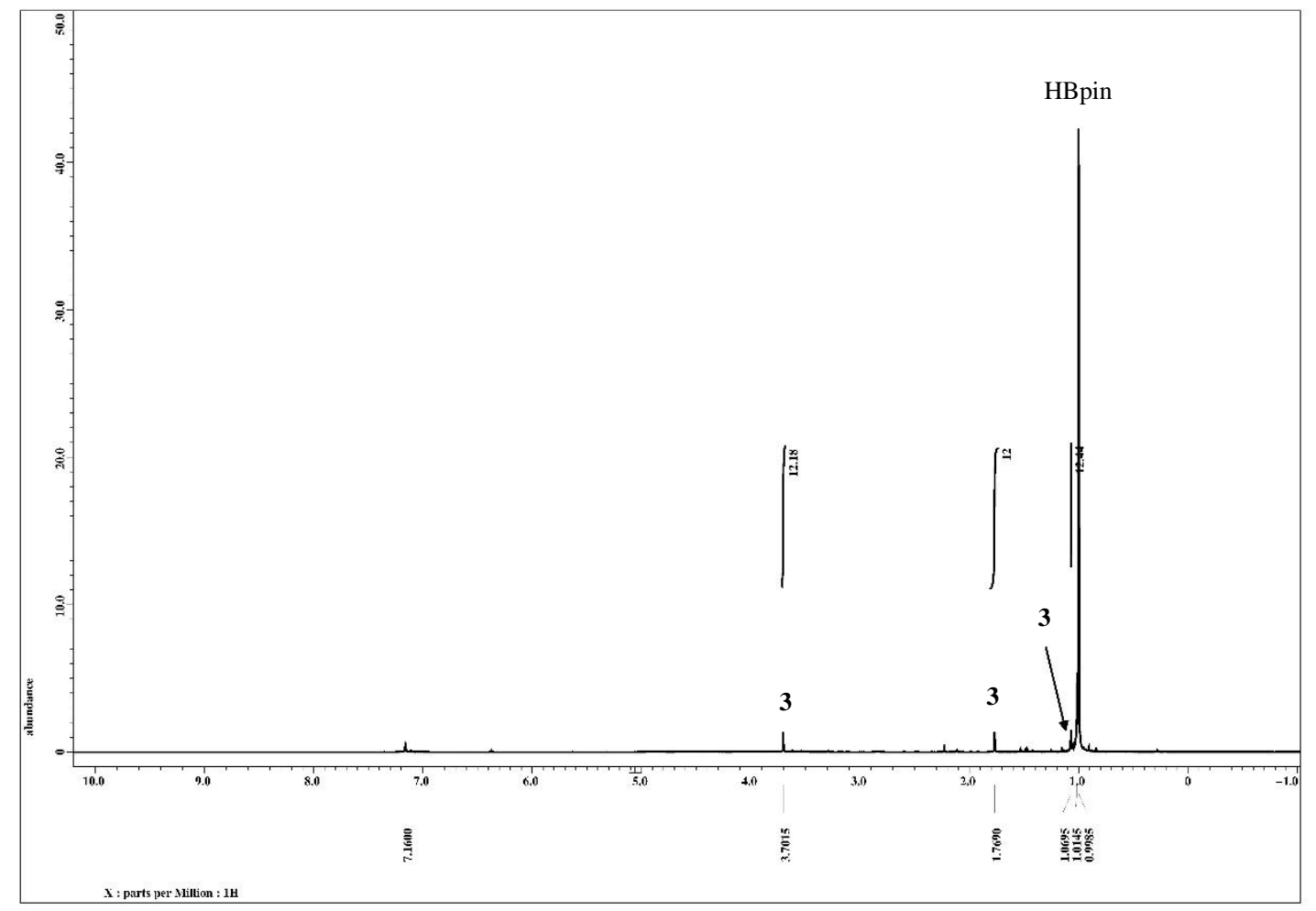

(a)

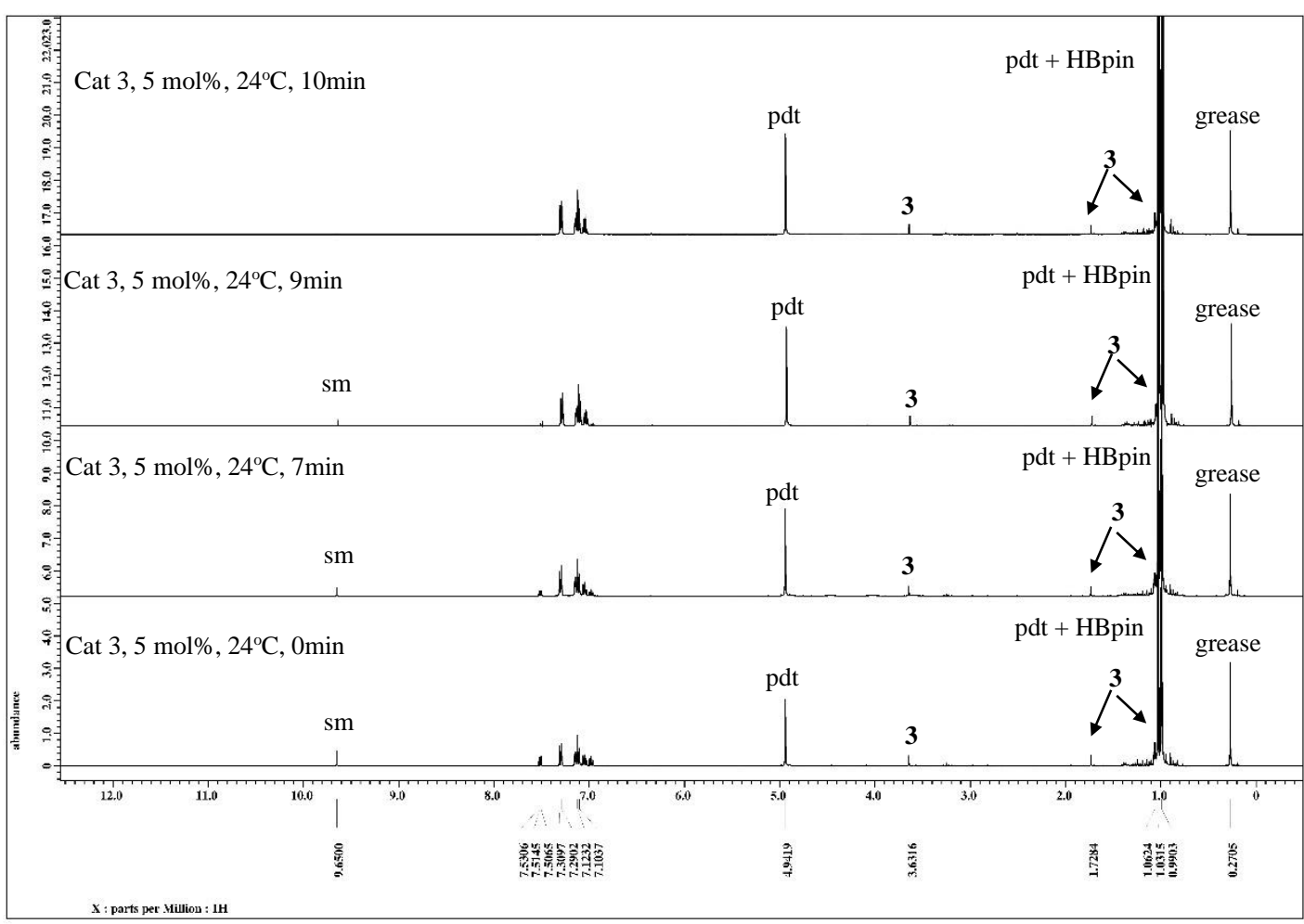

(b)

Figure S17. Stepwise hydroboration of benzaldehyde catalysed by $3\left(5 \mathrm{~mol} \%, 24{ }^{\circ} \mathrm{C}\right.$, in $\left.\mathrm{C}_{6} \mathrm{D}_{6}\right)$, monitored by ${ }^{1} \mathrm{H}$ NMR spectroscopy, (a) first step: addition of HBpin to $\mathbf{1}$ to afford $\mathbf{3}$ and (b) second step: addition of benzaldehyde. 


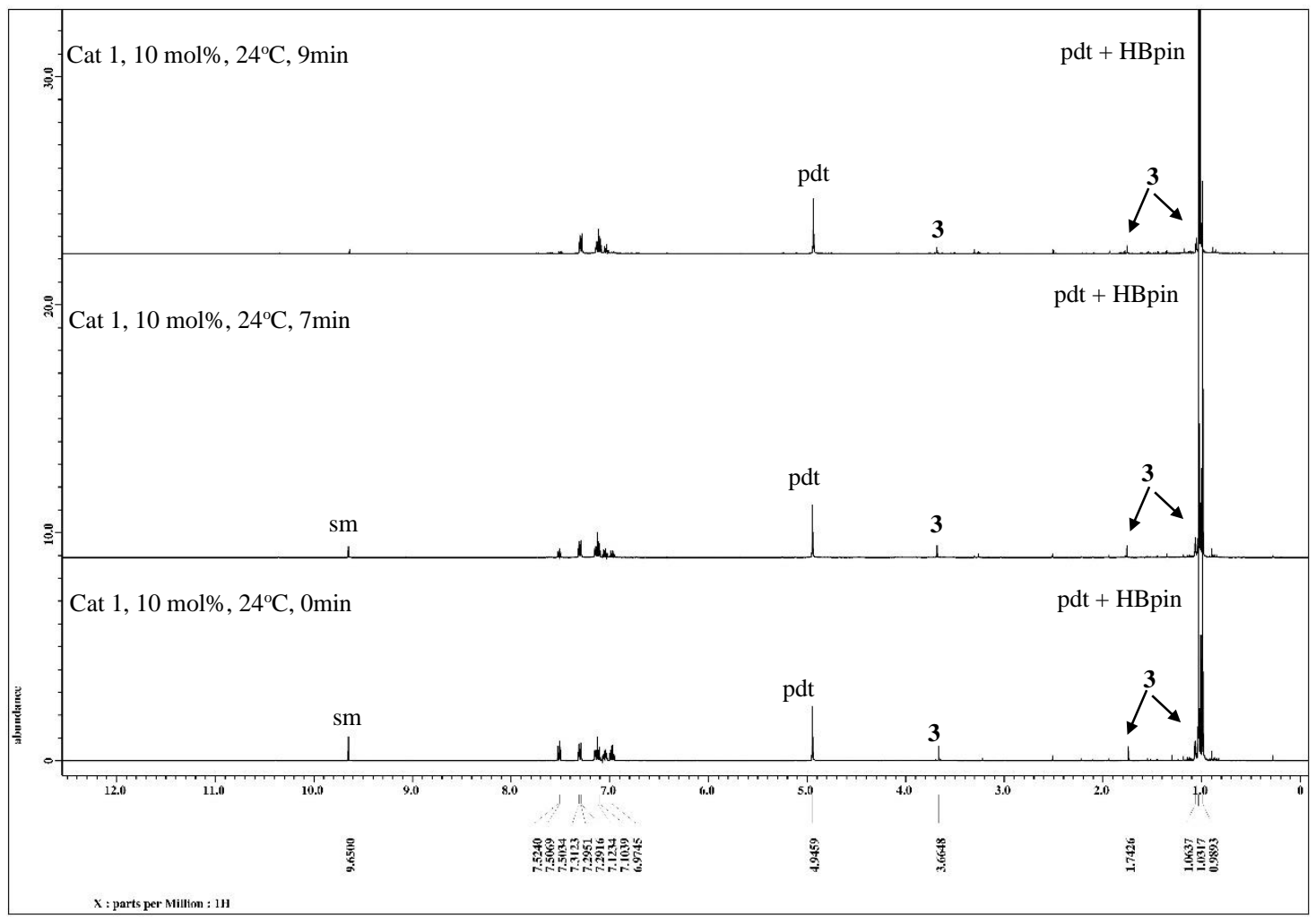

Figure S18. Timeline study of hydroboration of benzaldehyde $4 \mathbf{a}$ catalysed by $\mathbf{1}$ (10 mol \%, $24{ }^{\circ} \mathrm{C}$, in $\mathrm{C}_{6} \mathrm{D}_{6}$ ), monitored by ${ }^{1} \mathrm{H}$ NMR spectroscopy. 

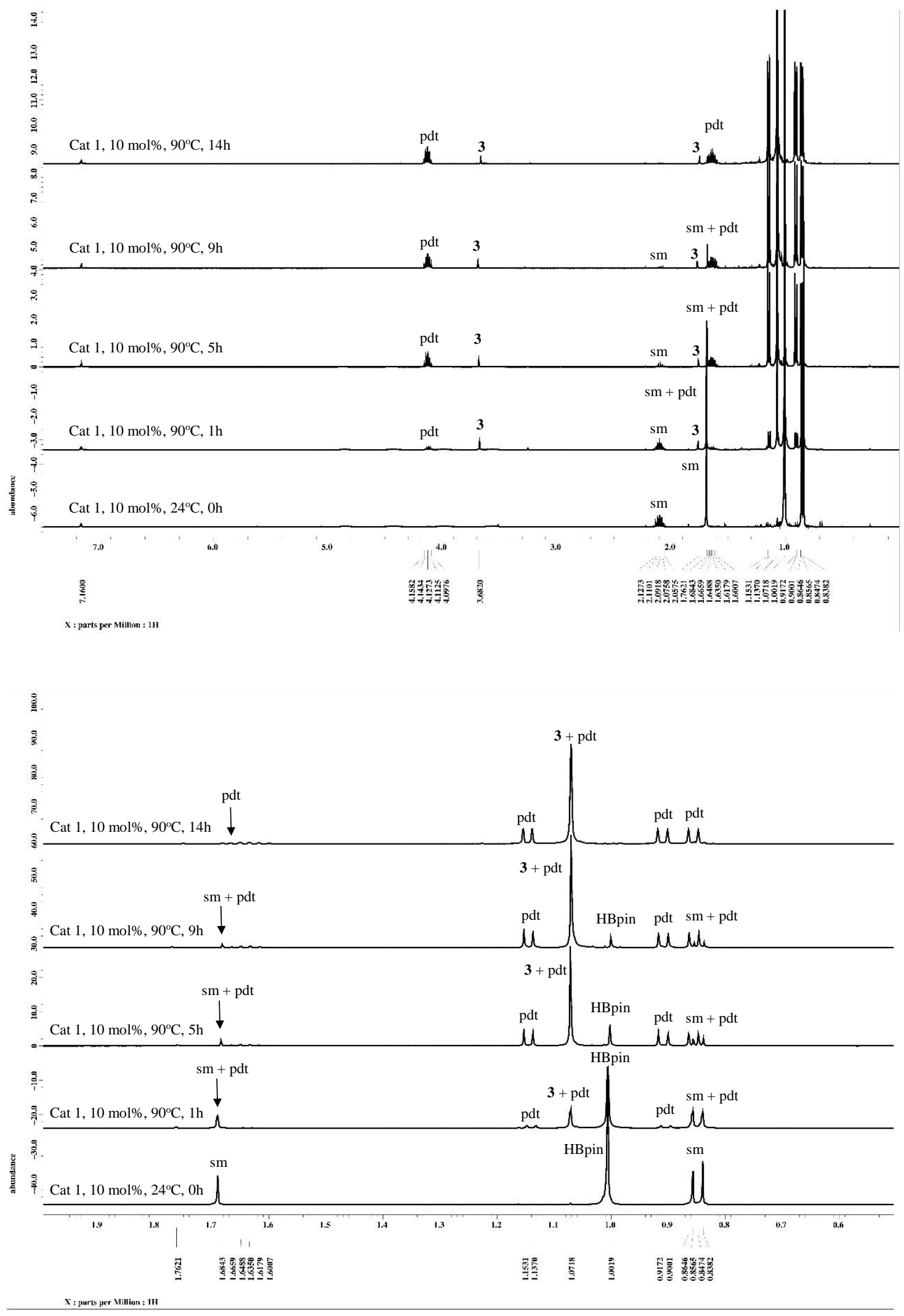

Figure S19. Timeline study of hydroboration of 3-methyl-2-butanone 6e catalysed by 1 (10 mol \%, $90{ }^{\circ} \mathrm{C}$, in $\mathrm{C}_{6} \mathrm{D}_{6}$ ), monitored by ${ }^{1} \mathrm{H}$ NMR spectroscopy. 

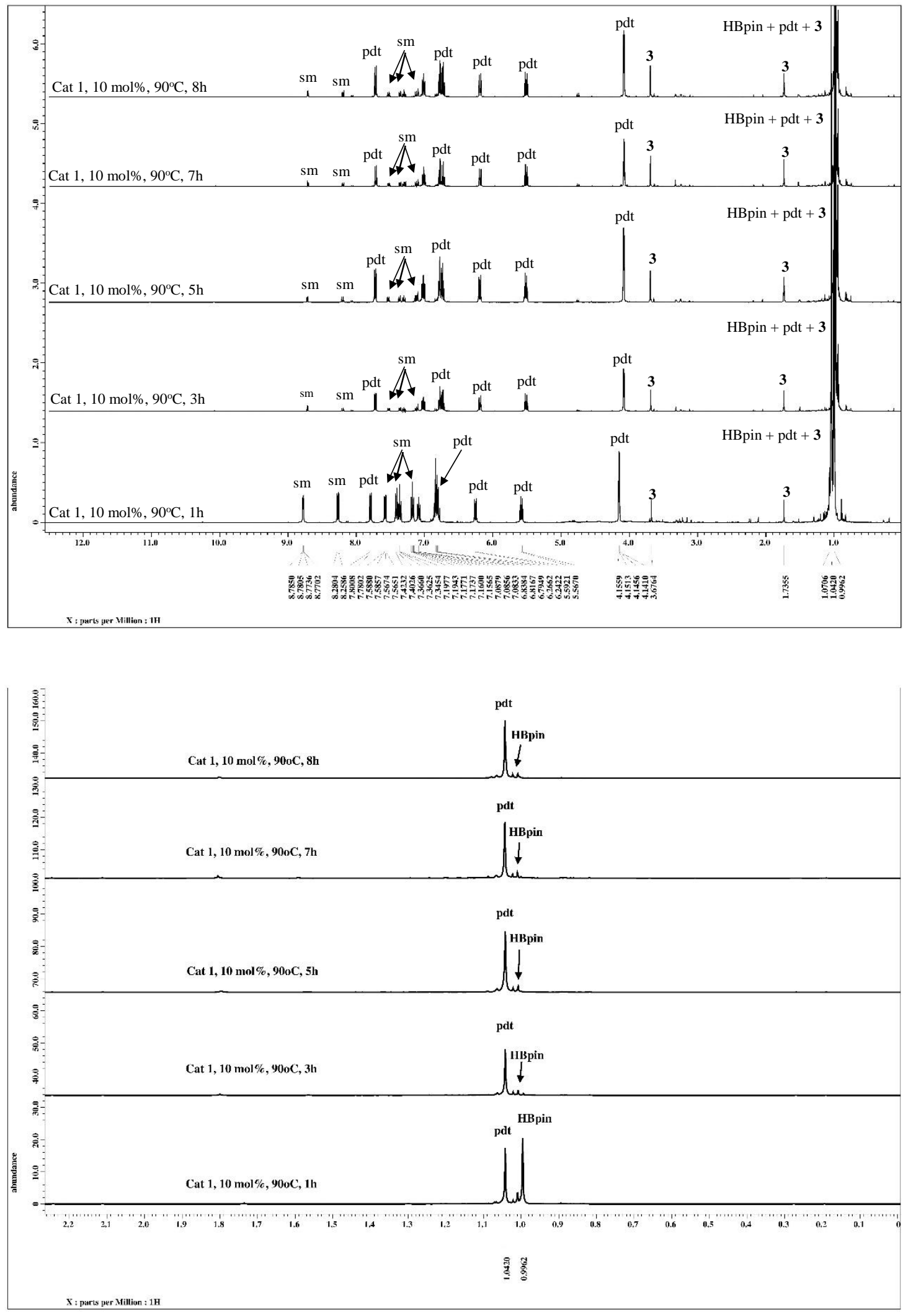

Figure S20. Timeline study of hydroboration of quinoline $8 \mathbf{d}$ catalysed by $\mathbf{1}\left(10 \mathrm{~mol} \%, 90{ }^{\circ} \mathrm{C}\right.$, in $\mathrm{C}_{6} \mathrm{D}_{6}$ ), monitored by ${ }^{1} \mathrm{H}$ NMR spectroscopy. 

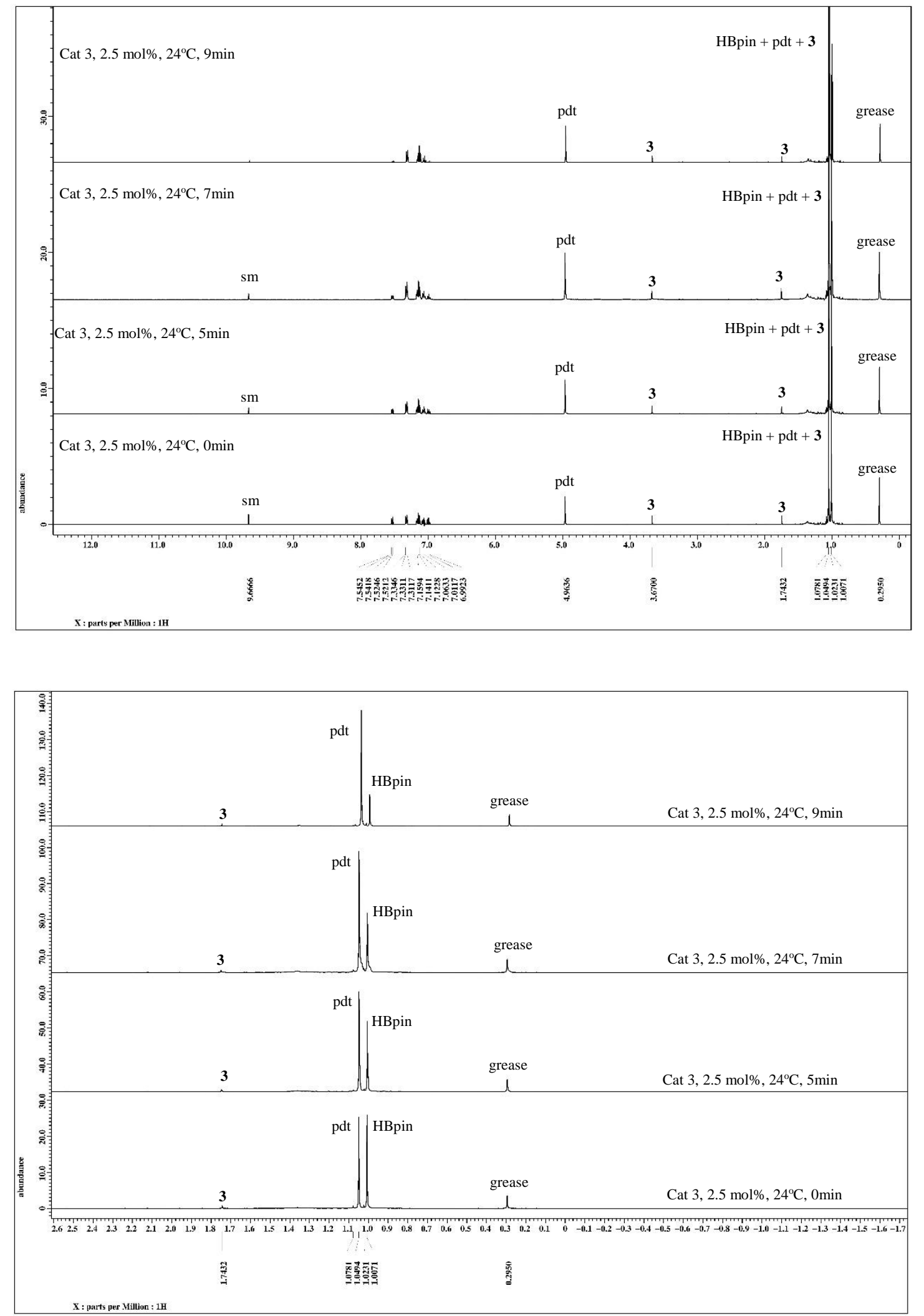

Figure S21. Timeline study of hydroboration of benzaldehyde $4 \mathbf{a}$ catalysed by 3 (2.5 mol \%, $24{ }^{\circ} \mathrm{C}$, in $\mathrm{C}_{6} \mathrm{D}_{6}$ ), monitored by ${ }^{1} \mathrm{H}$ NMR spectroscopy. 

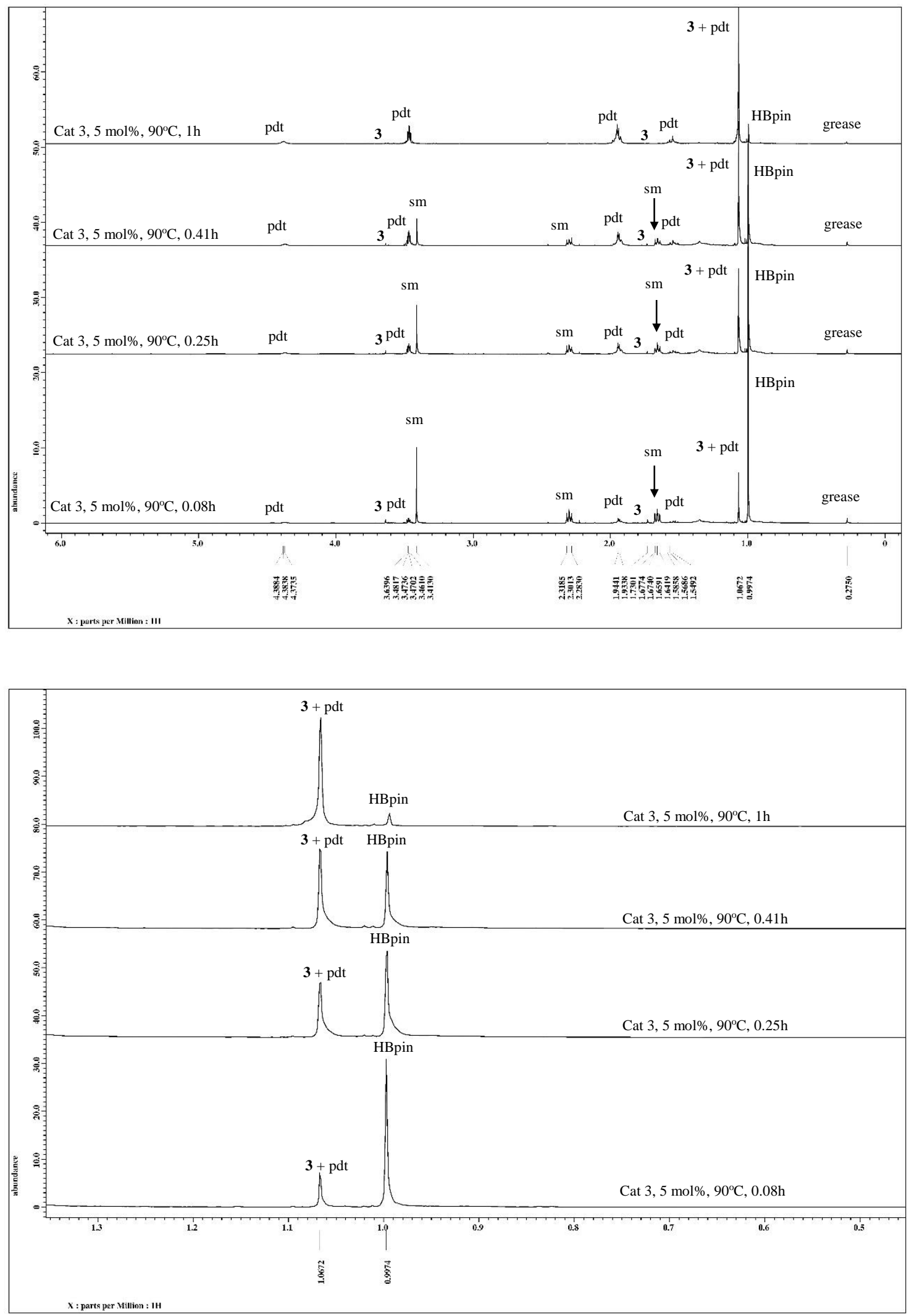

Figure S22. Timeline study of hydroboration of 1,4-dioxaspiro[4.5]decan-8-one $\mathbf{6 d}$ catalysed by $3\left(5 \mathrm{~mol} \%, 90{ }^{\circ} \mathrm{C}\right.$, in $\left.\mathrm{C}_{6} \mathrm{D}_{6}\right)$, monitored by ${ }^{1} \mathrm{H}$ NMR spectroscopy. 

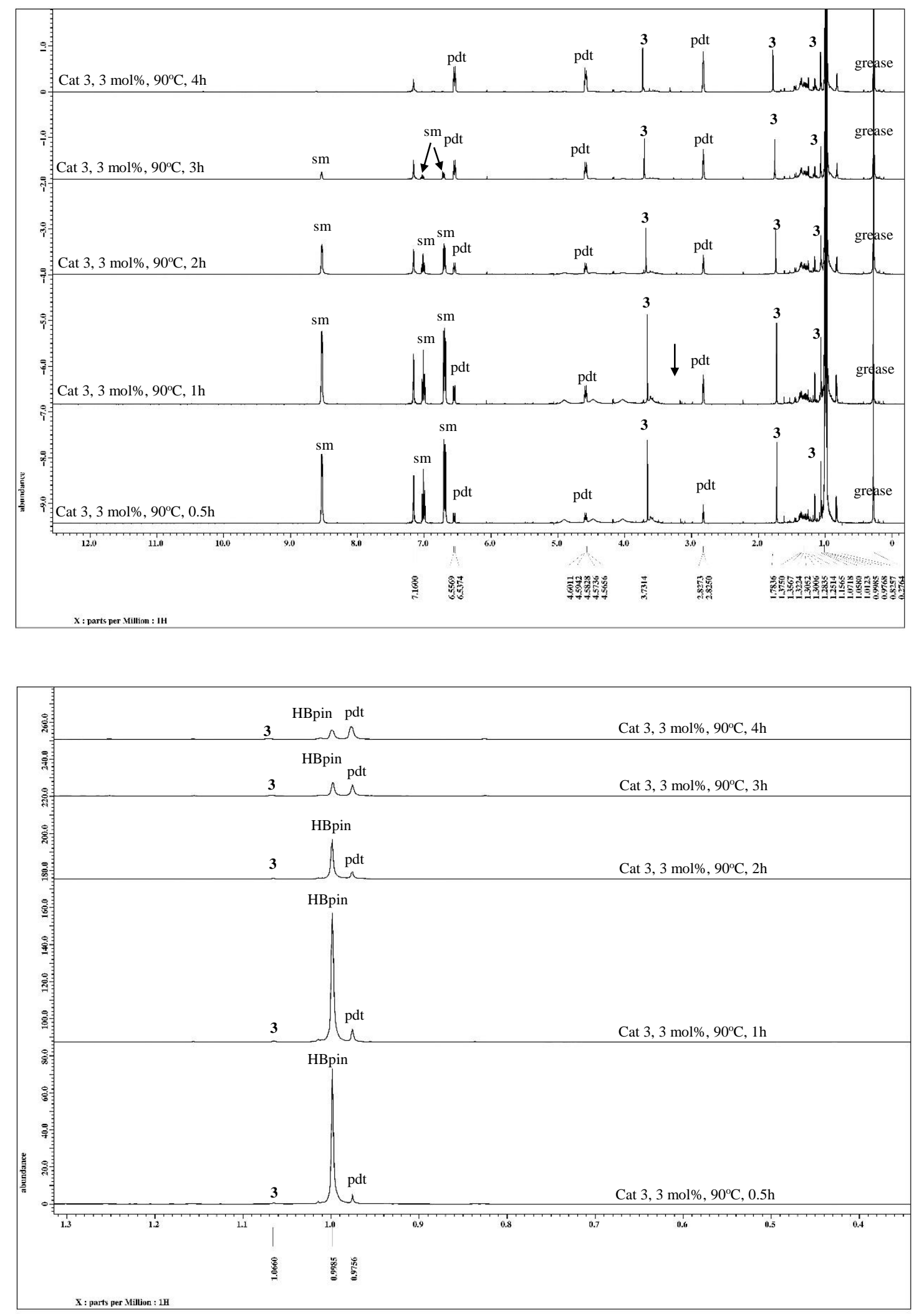

Figure S23. Timeline study of hydroboration of pyridine 8a catalysed by $\mathbf{3}$ (3 mol \%, $90{ }^{\circ} \mathrm{C}$, in $\mathrm{C}_{6} \mathrm{D}_{6}$ ), monitored by ${ }^{1} \mathrm{H}$ NMR spectroscopy. 


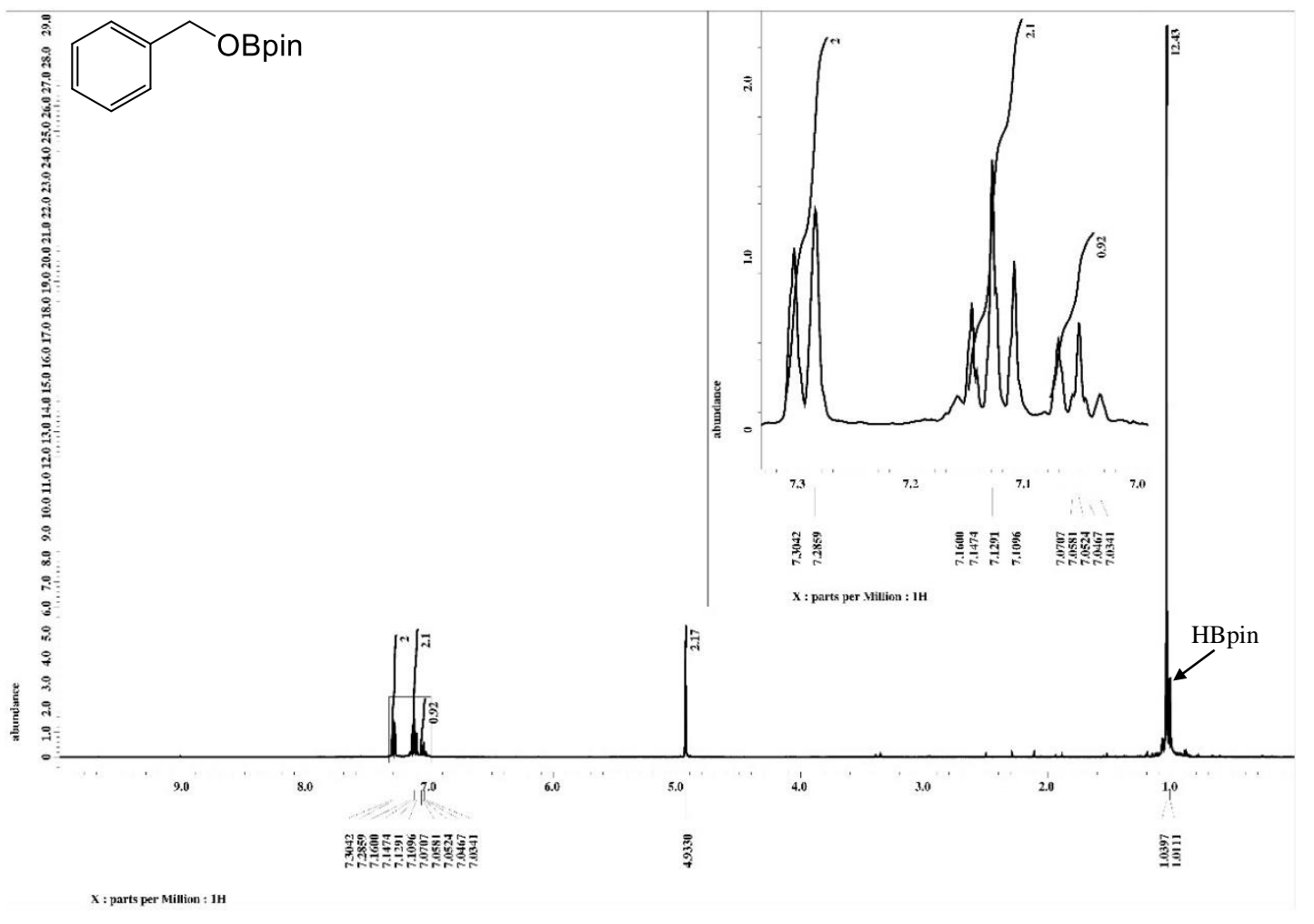

(a)

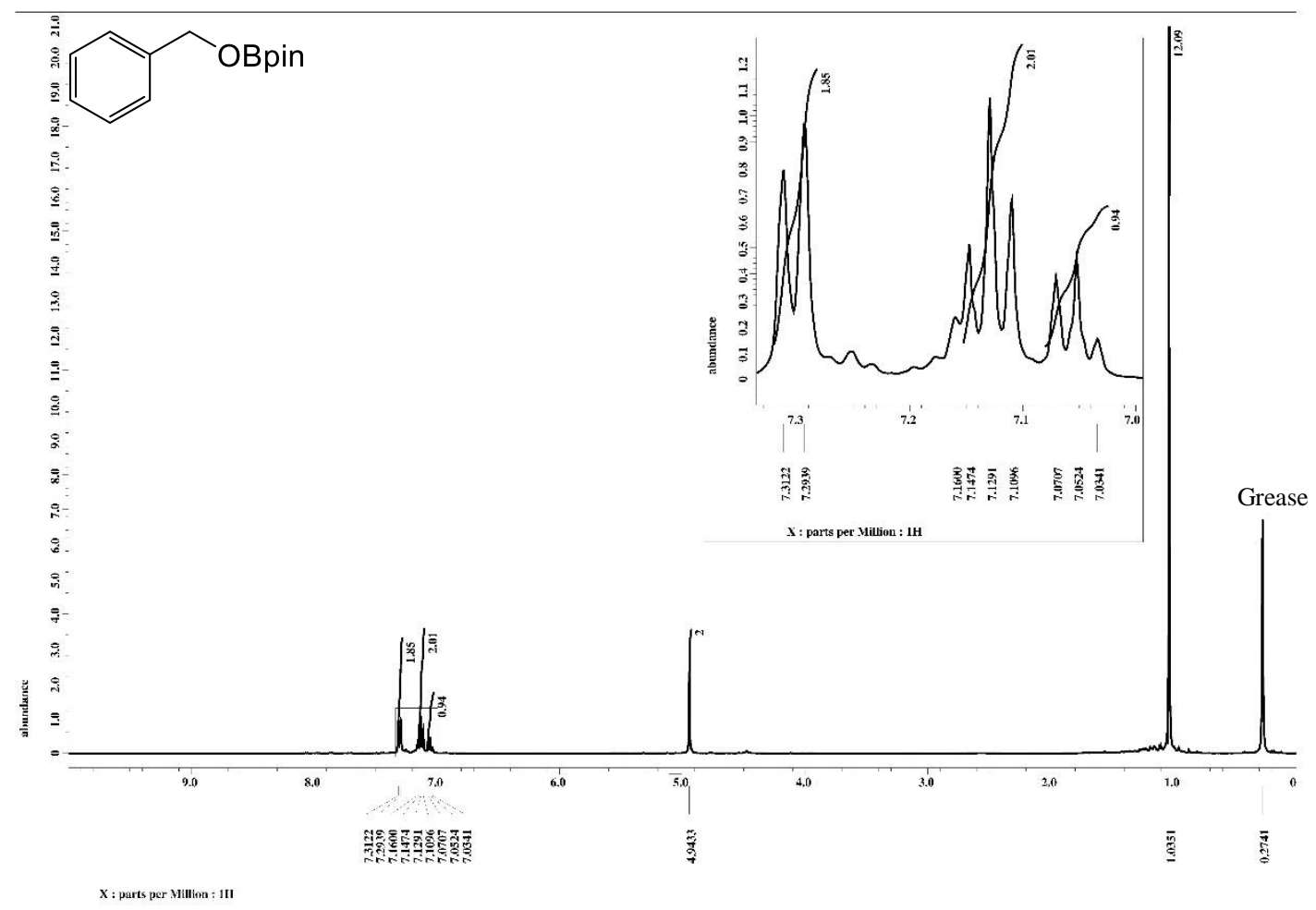

(b)

Figure S24. ${ }^{1} \mathrm{H}$ NMR spectrum of compound $5 \mathbf{a}$ (in $\mathrm{C}_{6} \mathrm{D}_{6}$ ), (a) crude solution and (b) isolated compound. 


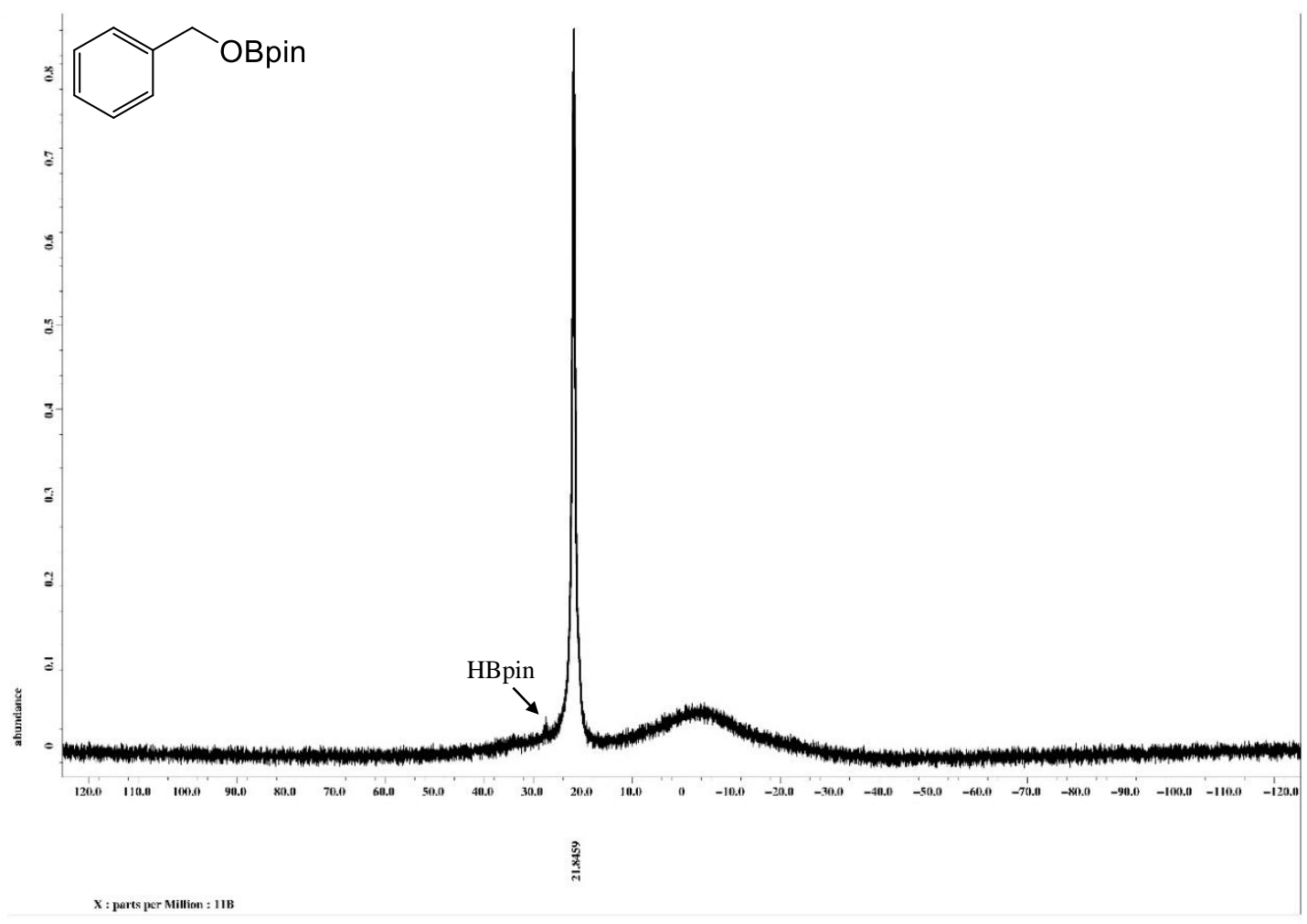

(a)

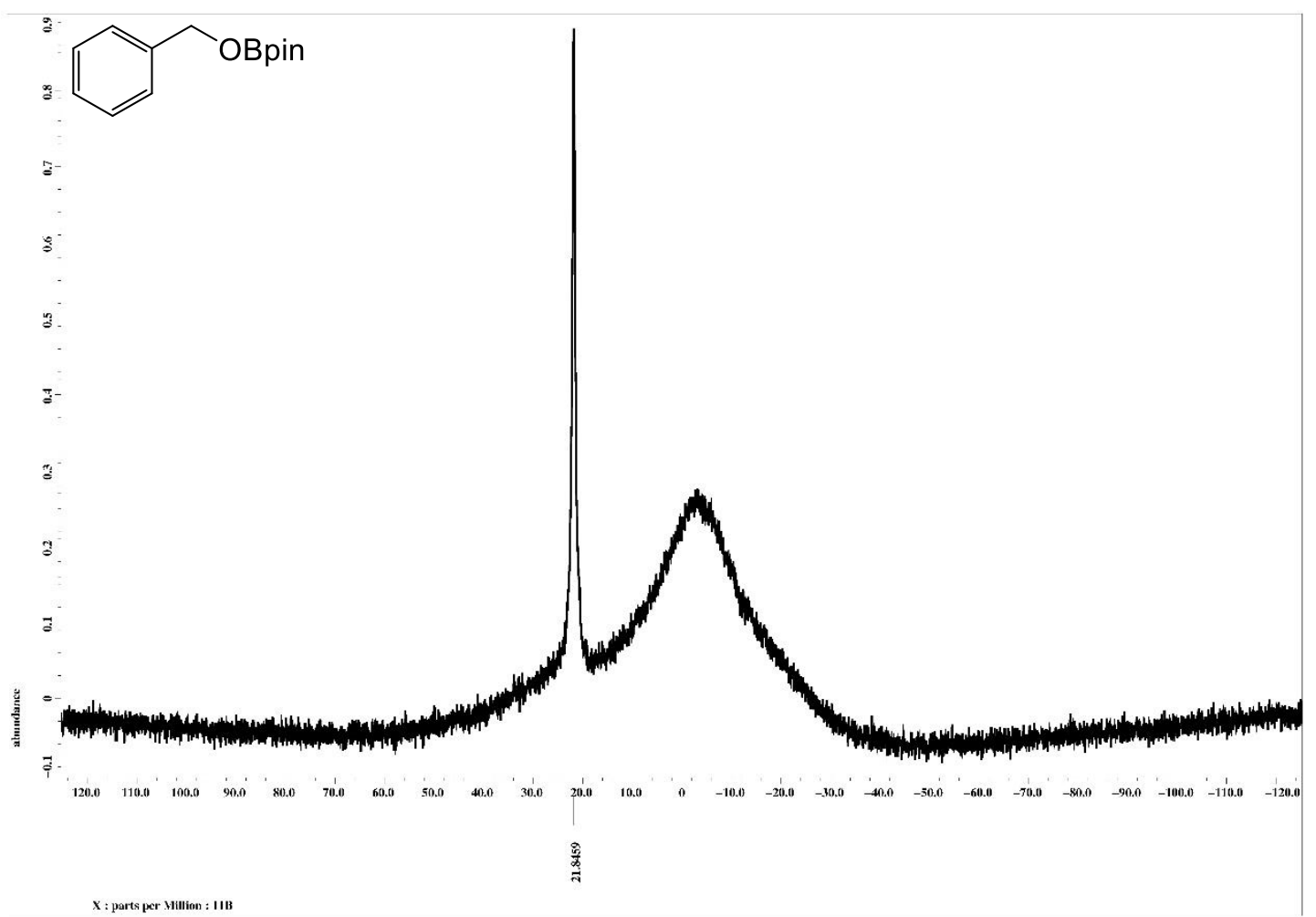

(b)

Figure S25. ${ }^{11} \mathrm{~B}\left\{{ }^{1} \mathrm{H}\right\}$ NMR spectrum of compound 5a (in $\mathrm{C}_{6} \mathrm{D}_{6}$ ), (a) crude solution and (b) isolated compound. 


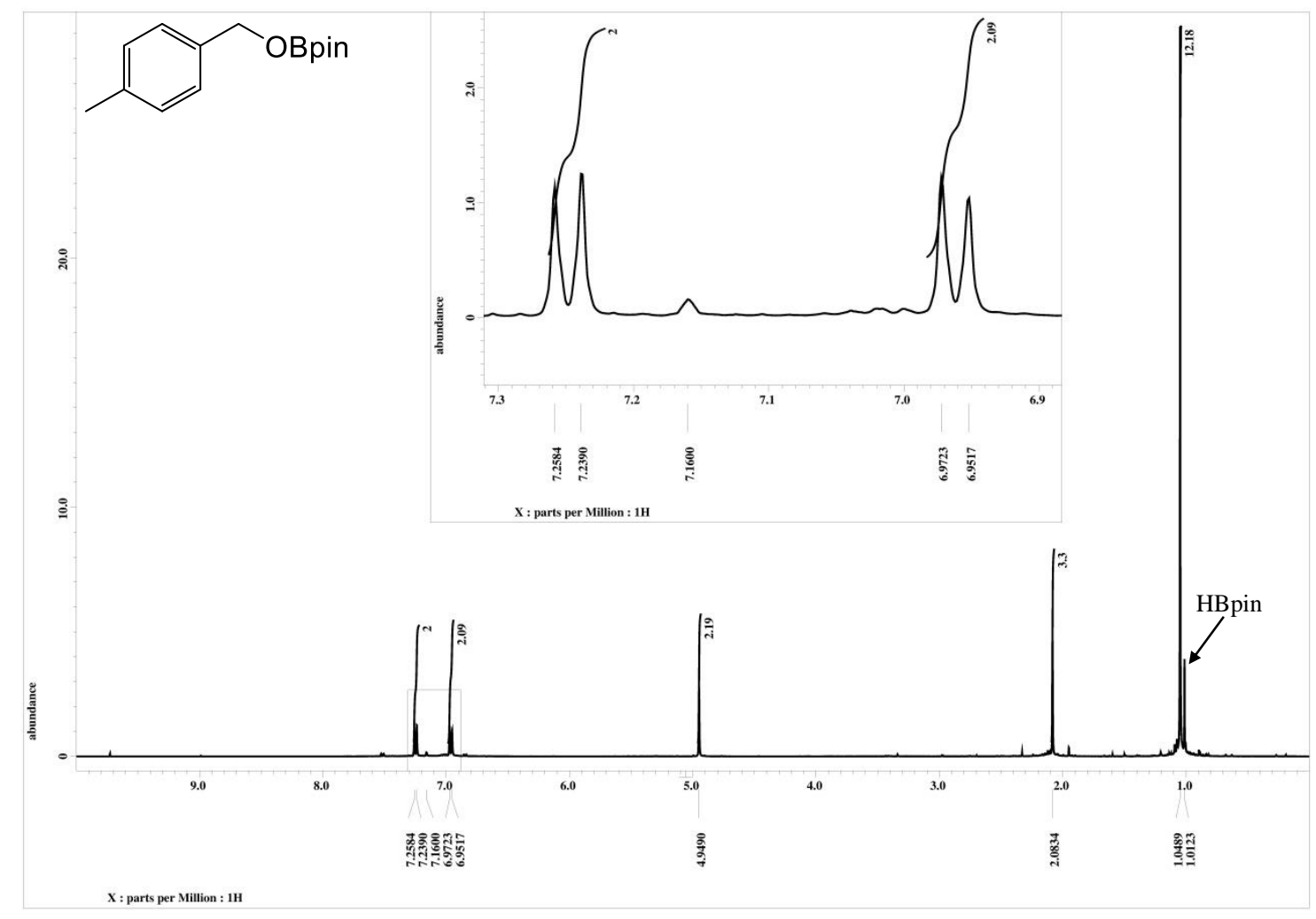

Figure S26. ${ }^{1} \mathrm{H}$ NMR spectrum of compound $\mathbf{5 b}$ (in $\mathrm{C}_{6} \mathrm{D}_{6}$ ).

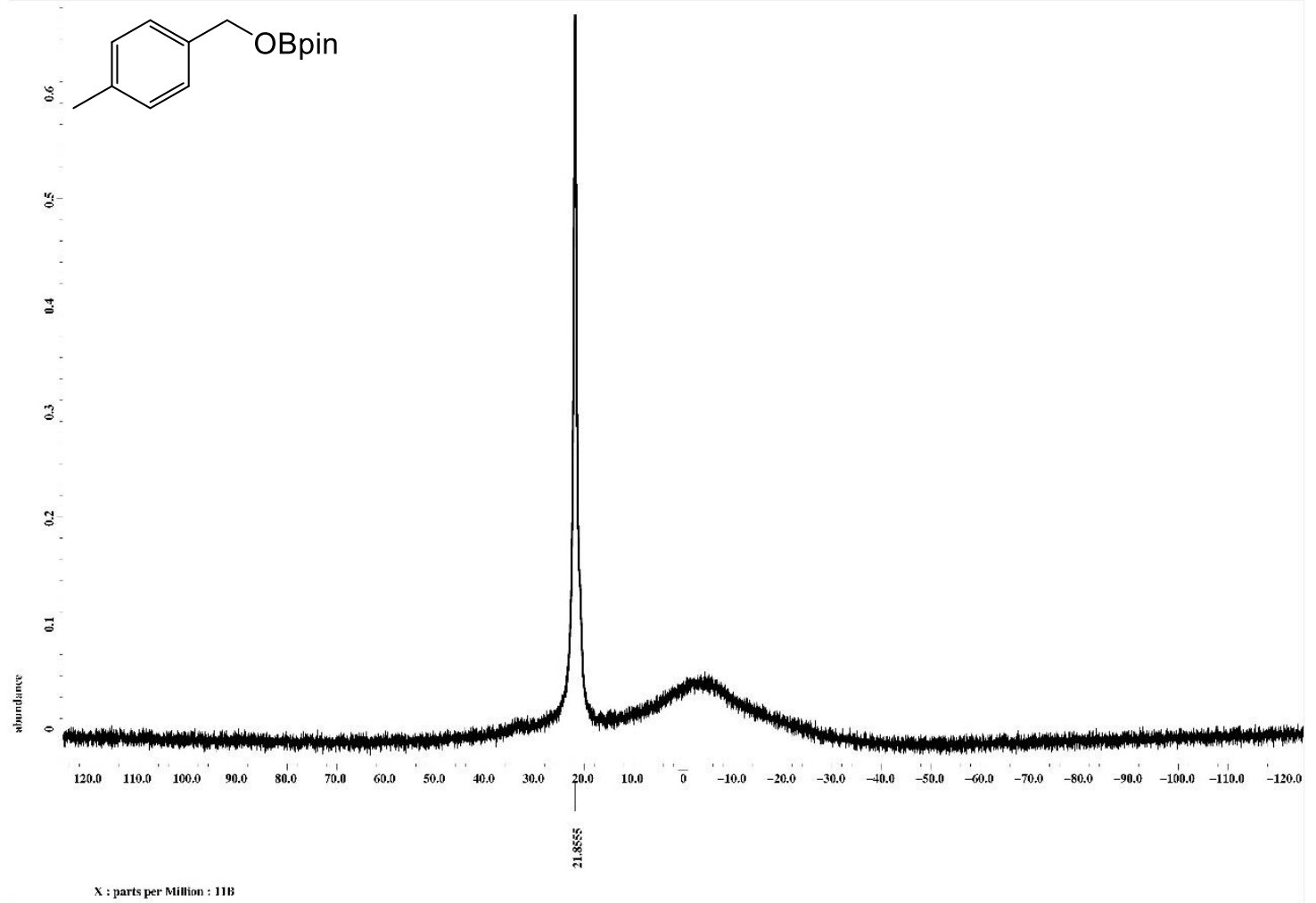

Figure S27. ${ }^{11} \mathrm{~B}\left\{{ }^{1} \mathrm{H}\right\}$ NMR spectrum of compound $\mathbf{5 b}$ (in $\mathrm{C}_{6} \mathrm{D}_{6}$ ). 


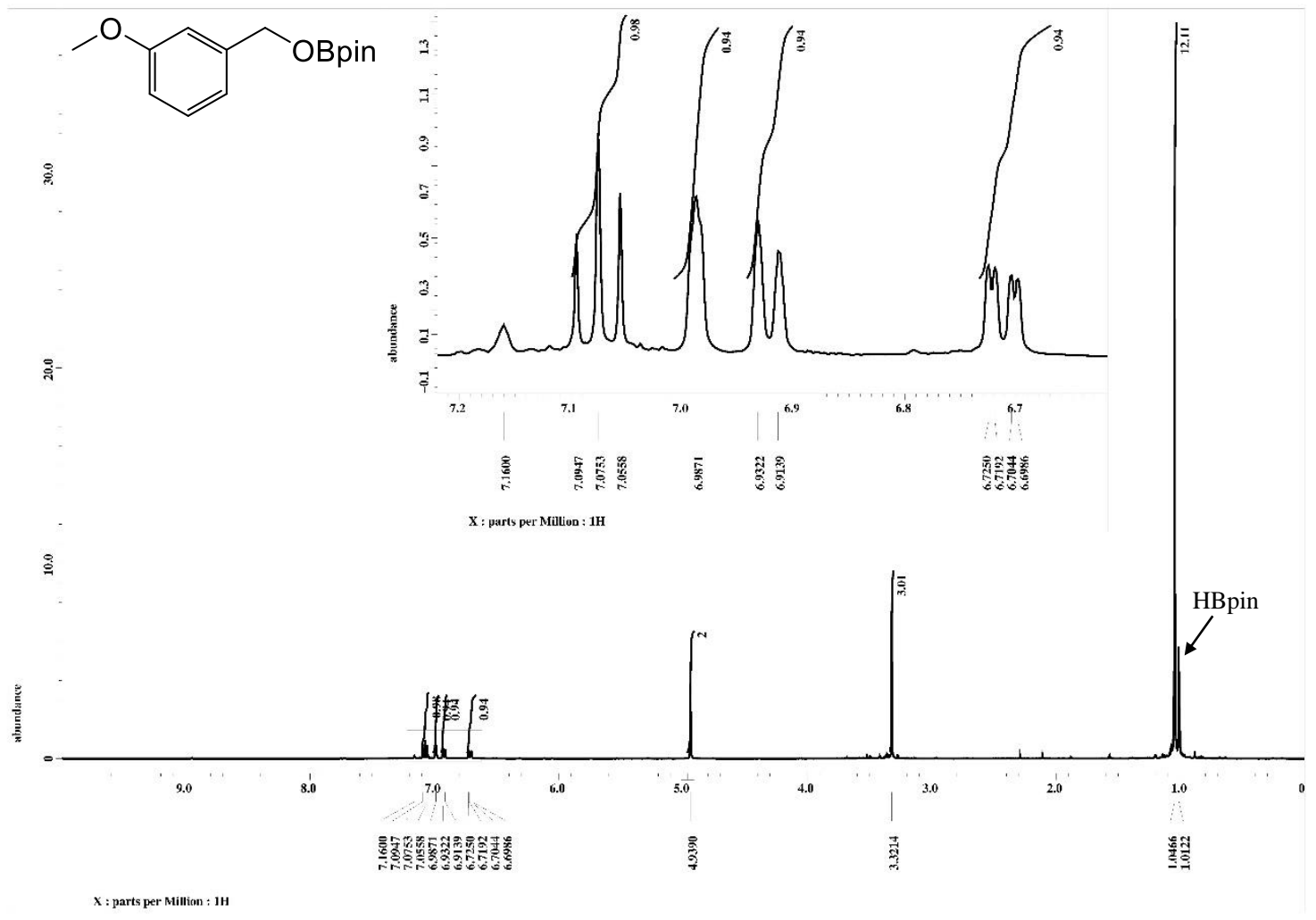

Figure S28. ${ }^{1} \mathrm{H}$ NMR spectrum of compound $5 \mathbf{c}$ (in $\mathrm{C}_{6} \mathrm{D}_{6}$ ).

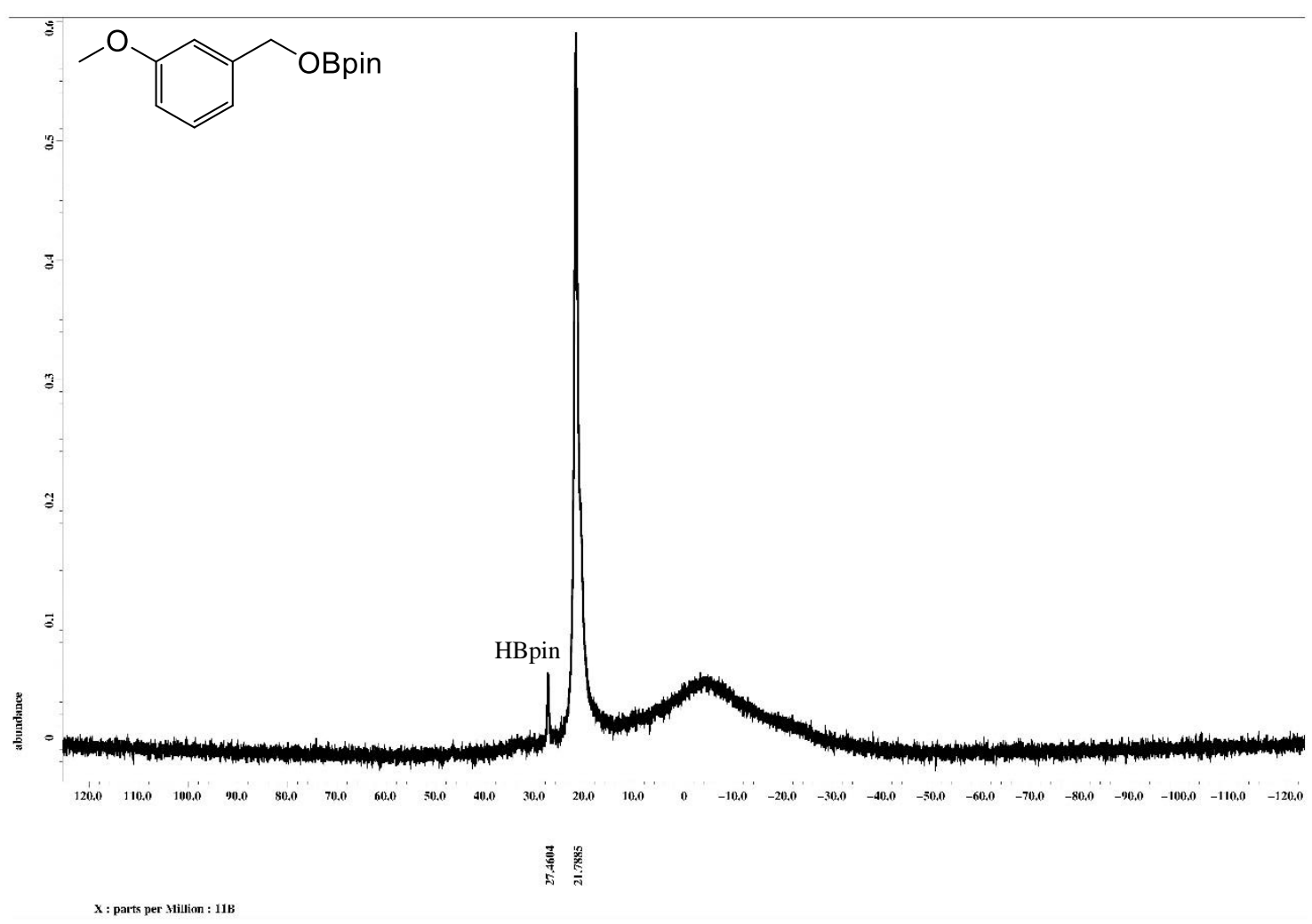

Figure S29. ${ }^{11} \mathrm{~B}\left\{{ }^{1} \mathrm{H}\right\}$ NMR spectrum of compound $\mathbf{5 c}\left(\right.$ in $\left.\mathrm{C}_{6} \mathrm{D}_{6}\right)$. 


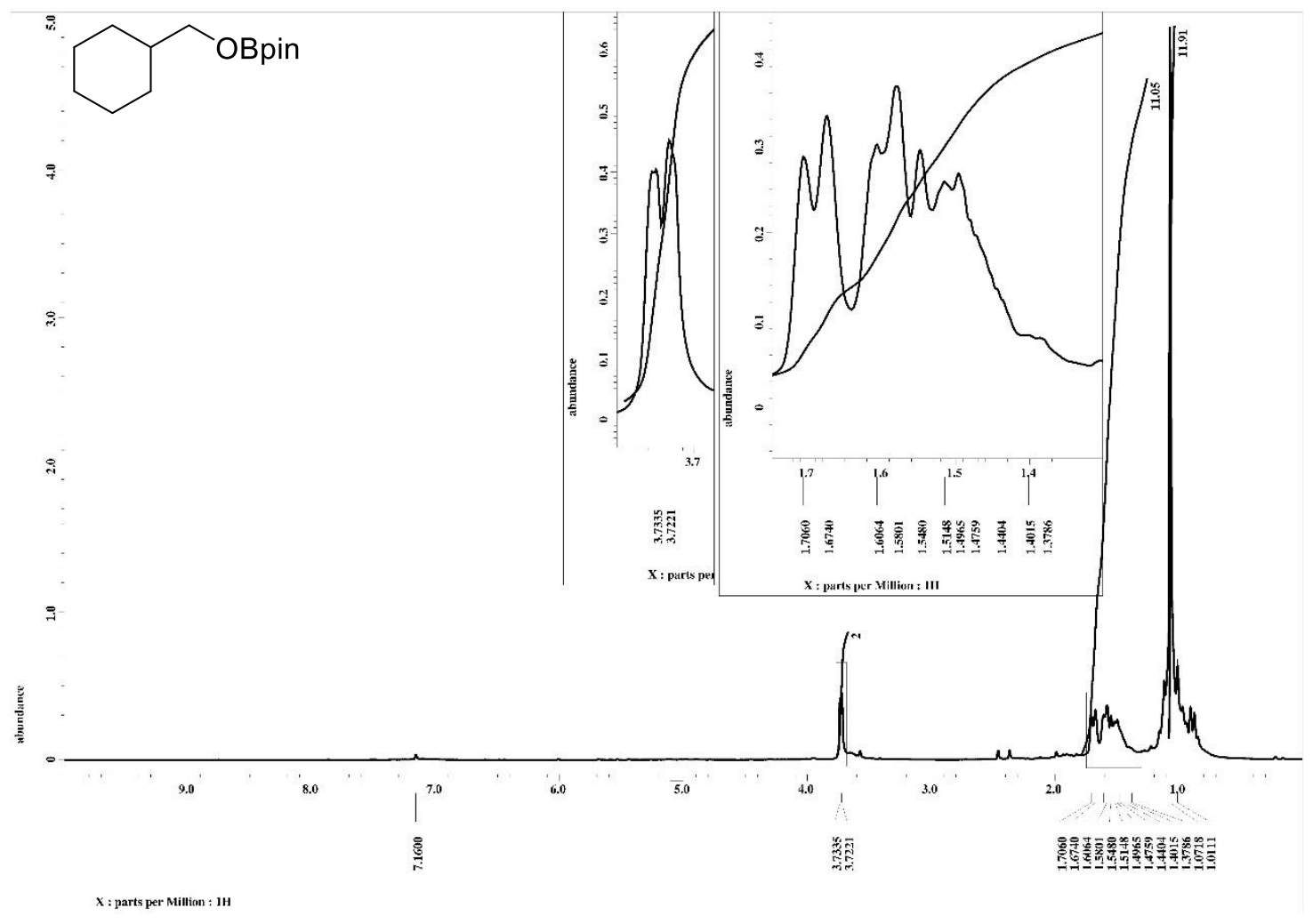

Figure S30. ${ }^{1} \mathrm{H}$ NMR spectrum of compound $5 \mathbf{d}$ (in $\mathrm{C}_{6} \mathrm{D}_{6}$ ).

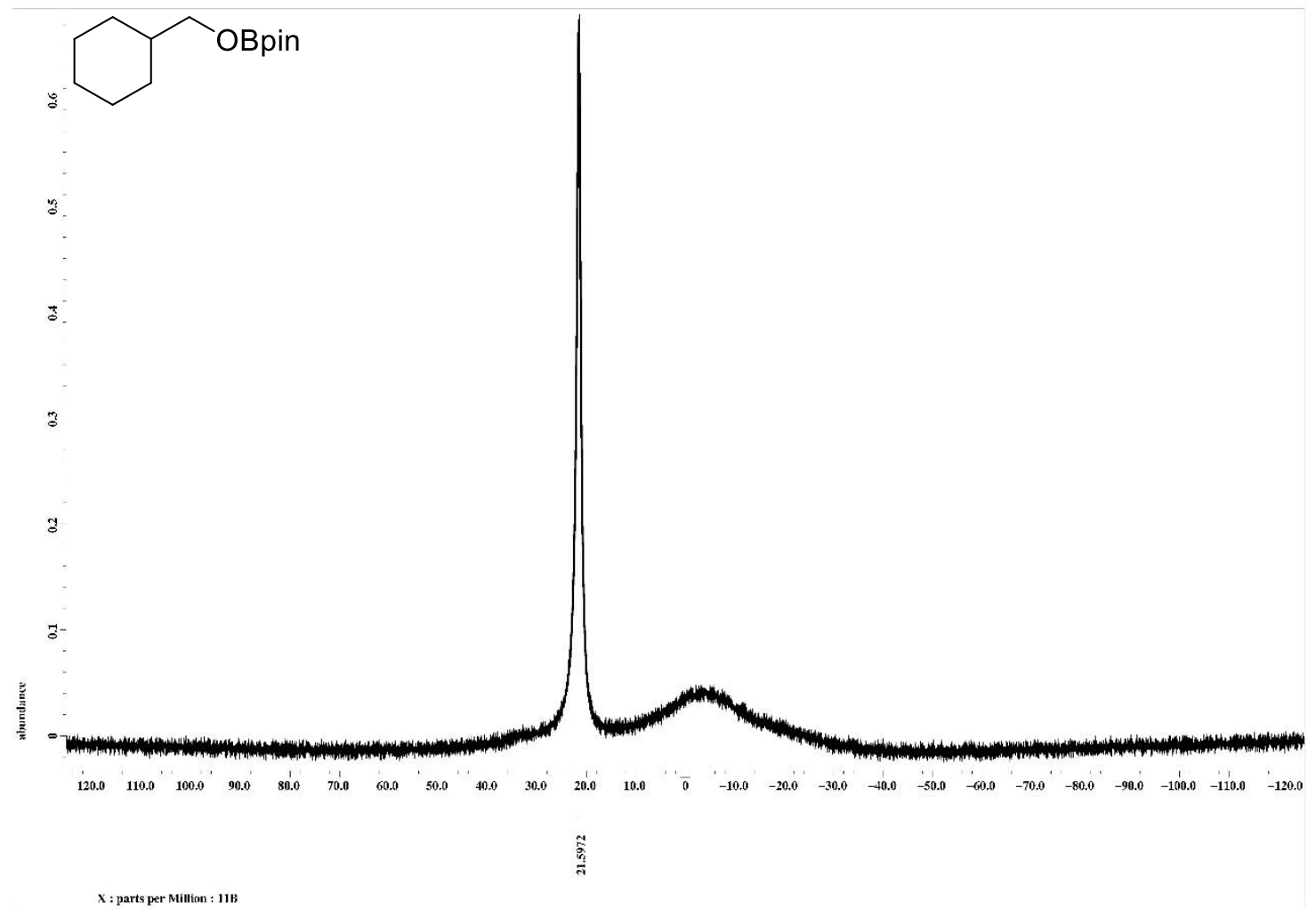

Figure S31. ${ }^{11} \mathrm{~B}\left\{{ }^{1} \mathrm{H}\right\}$ NMR spectrum of compound $\mathbf{5 d}$ (in $\mathrm{C}_{6} \mathrm{D}_{6}$ ). 
<smiles>COC(=O)c1ccc(C[R8]c2ccccc2)cc1</smiles>

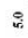

;

$8-$

s.

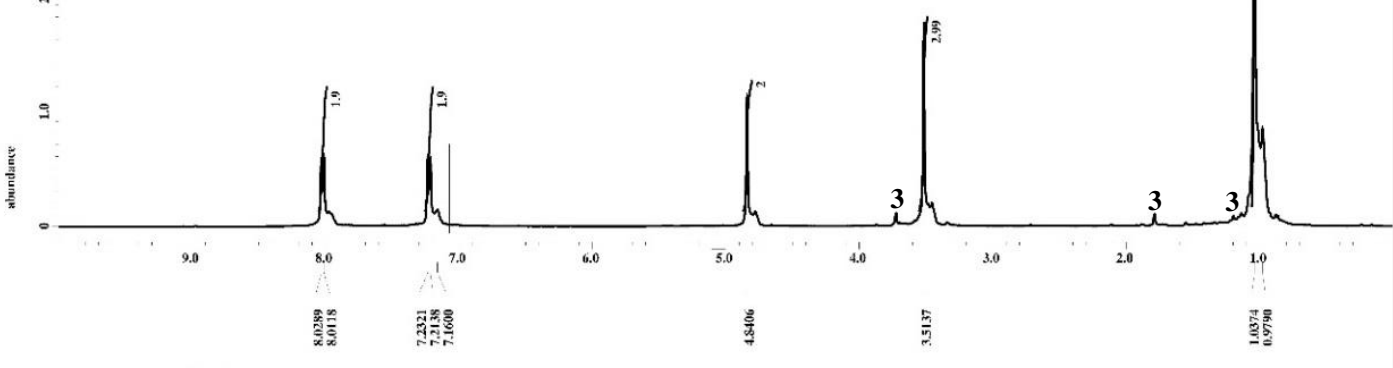

$\mathrm{X} ;$ parts per Millian : IH

(a)

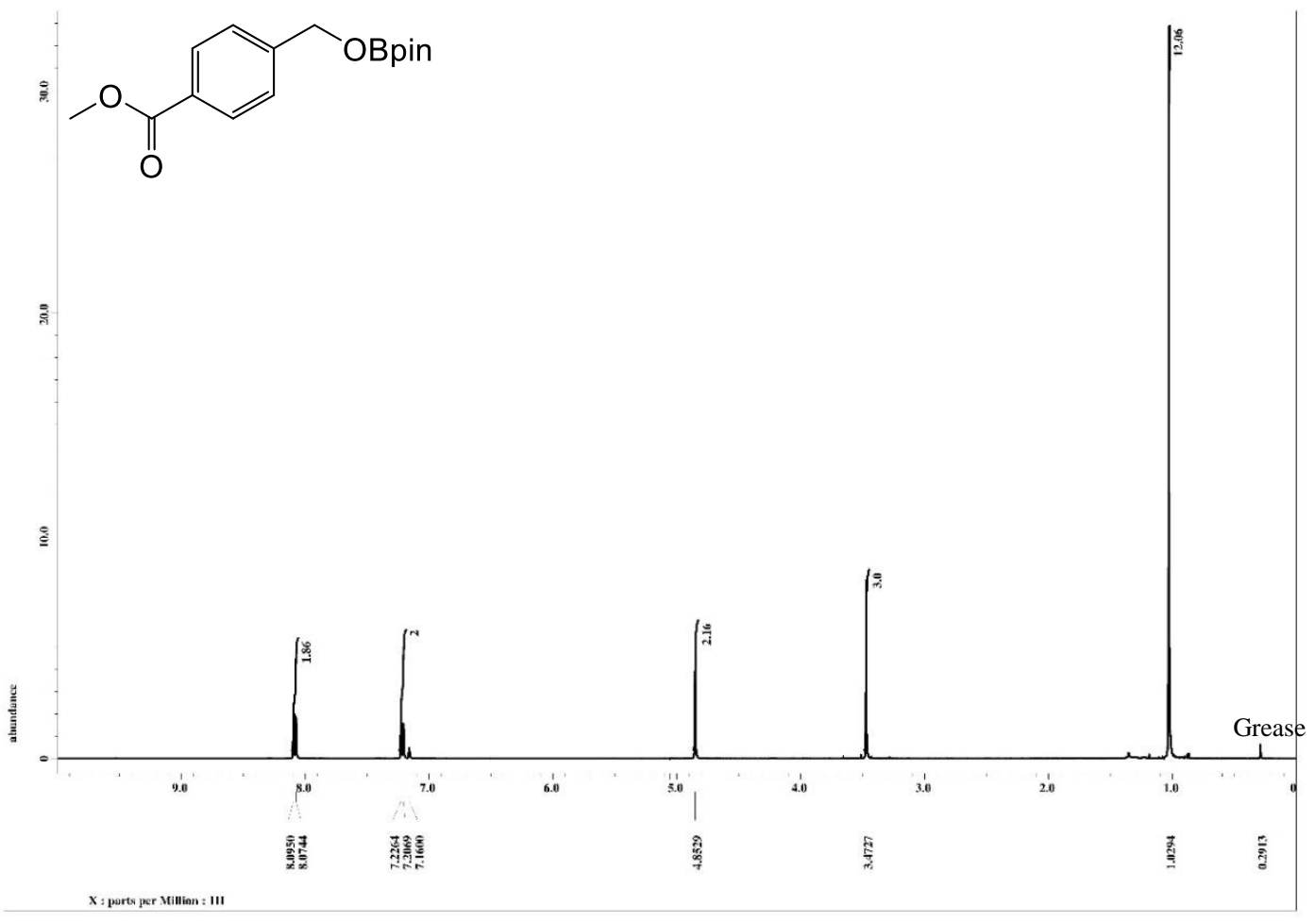

(b)

Figure S32. ${ }^{1} \mathrm{H}$ NMR spectrum of compound 5e (in $\mathrm{C}_{6} \mathrm{D}_{6}$ ), (a) crude solution and (b) isolated compound.

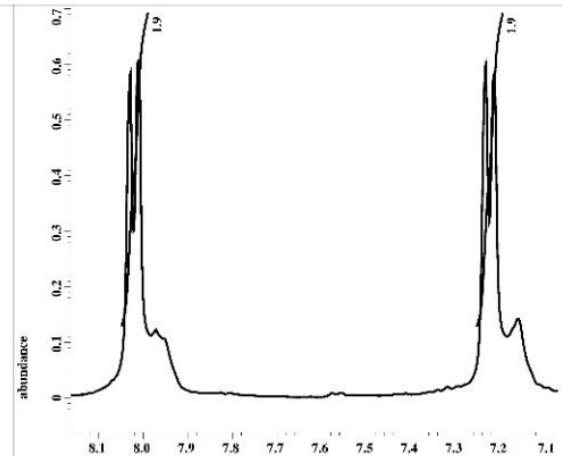

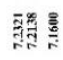

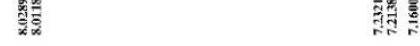

$X$ : parts per Milliwa : $1 H$

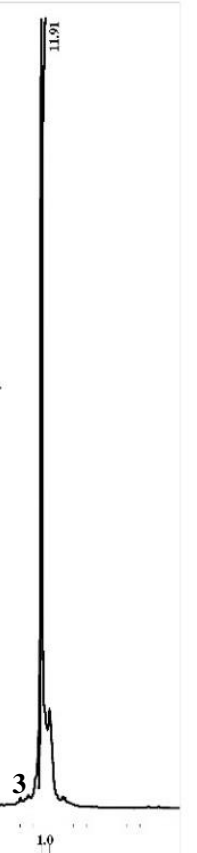




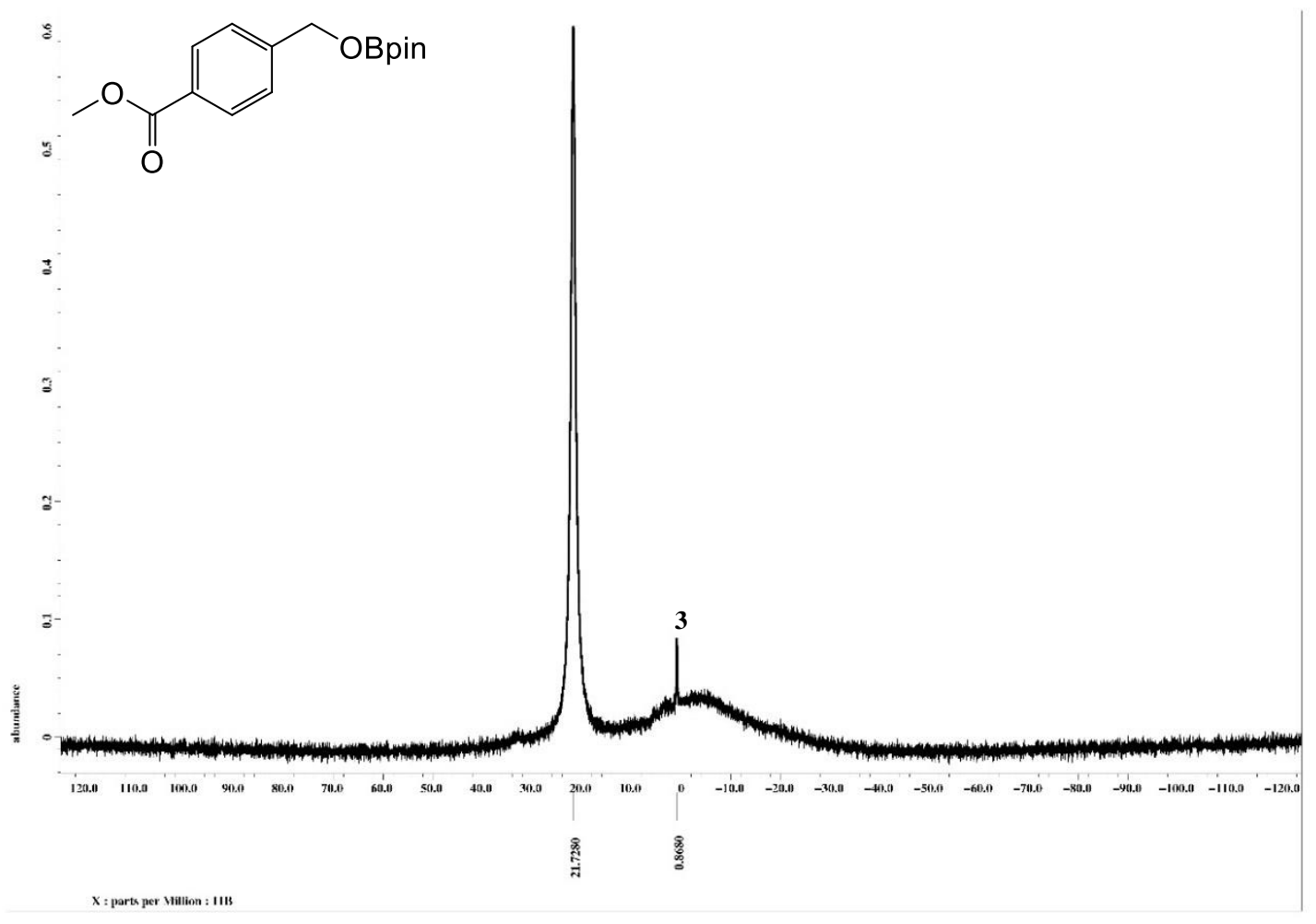

(a)

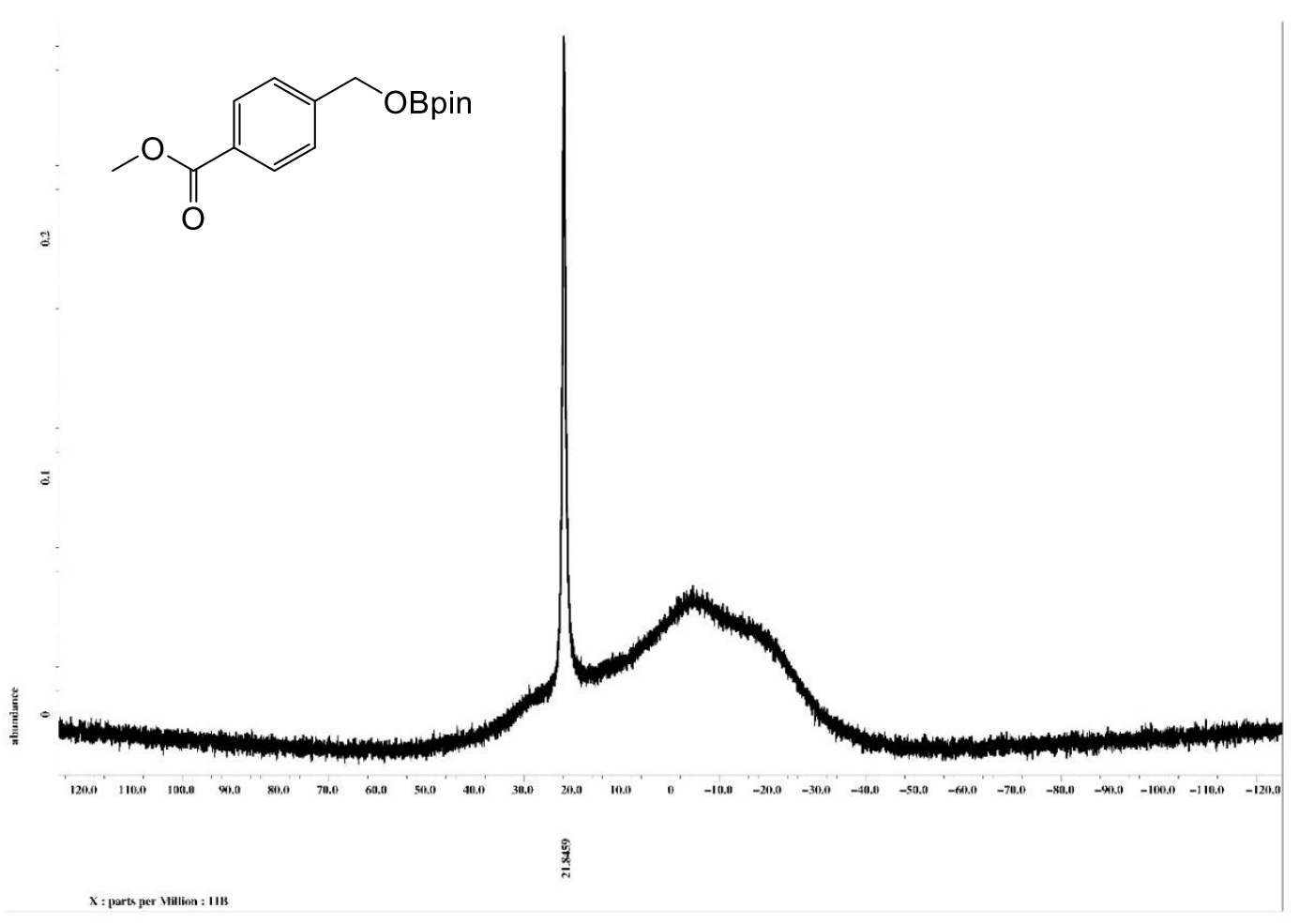

(b)

Figure S33. ${ }^{11} \mathrm{~B}\left\{{ }^{1} \mathrm{H}\right\}$ NMR spectrum of compound 5e (in $\mathrm{C}_{6} \mathrm{D}_{6}$ ), (a) crude solution and (b) isolated compound. 


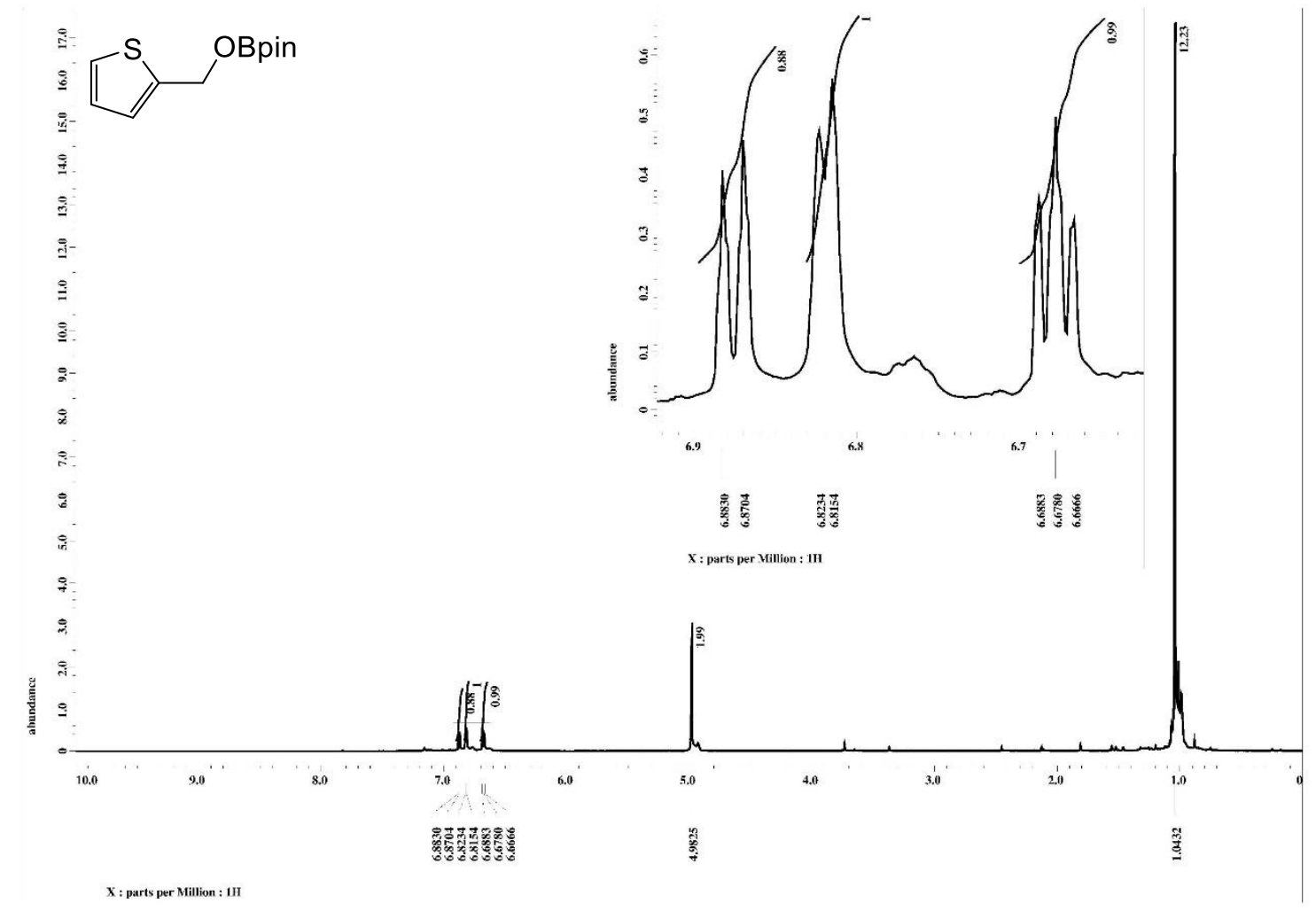

Figure S34. ${ }^{1} \mathrm{H}$ NMR spectrum of compound $\mathbf{5 f}$ (in $\mathrm{C}_{6} \mathrm{D}_{6}$ ).

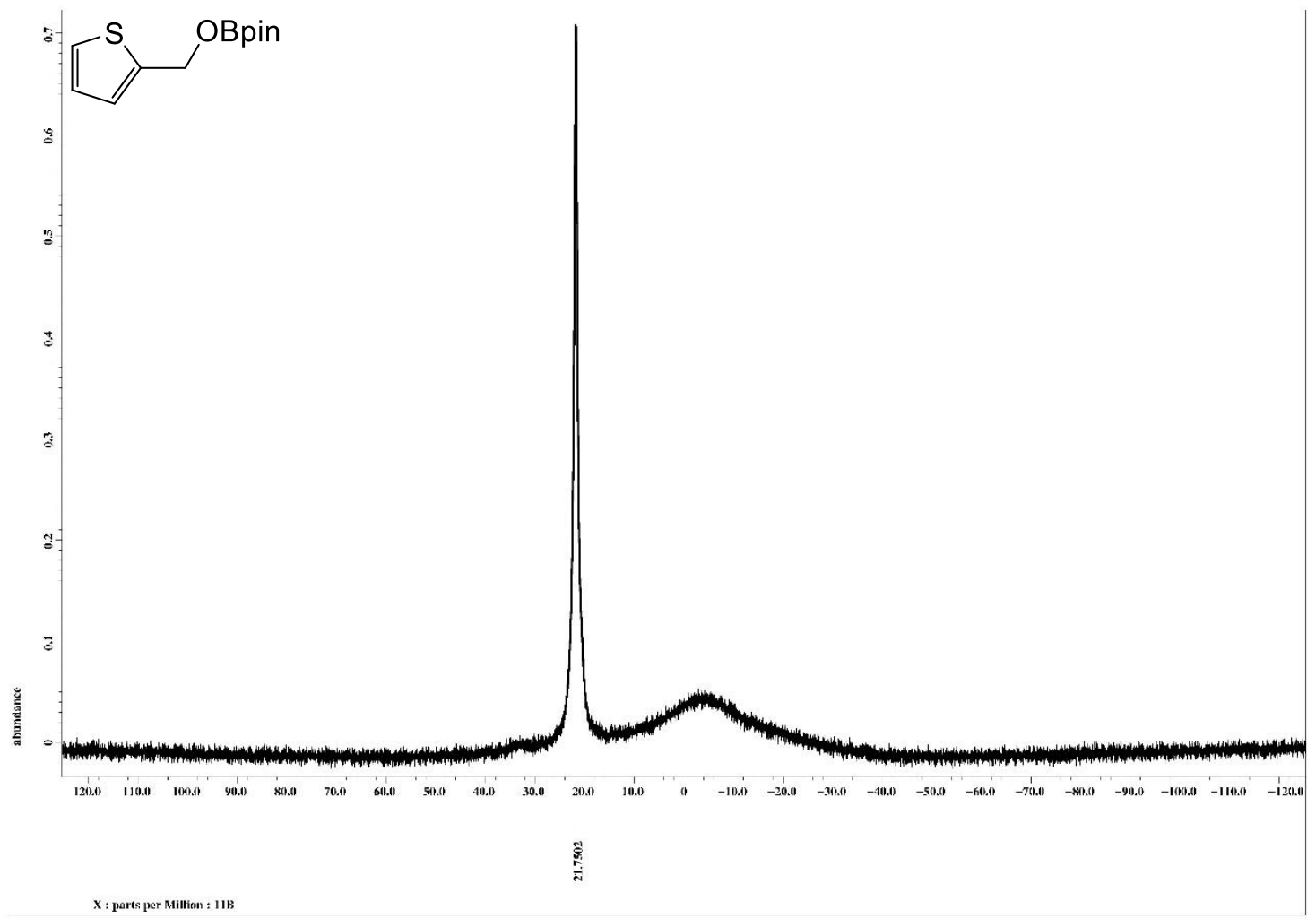

Figure S35. ${ }^{11} \mathrm{~B}\left\{{ }^{1} \mathrm{H}\right\}$ NMR spectrum of compound $\mathbf{5 f}$ (in $\mathrm{C}_{6} \mathrm{D}_{6}$ ). 


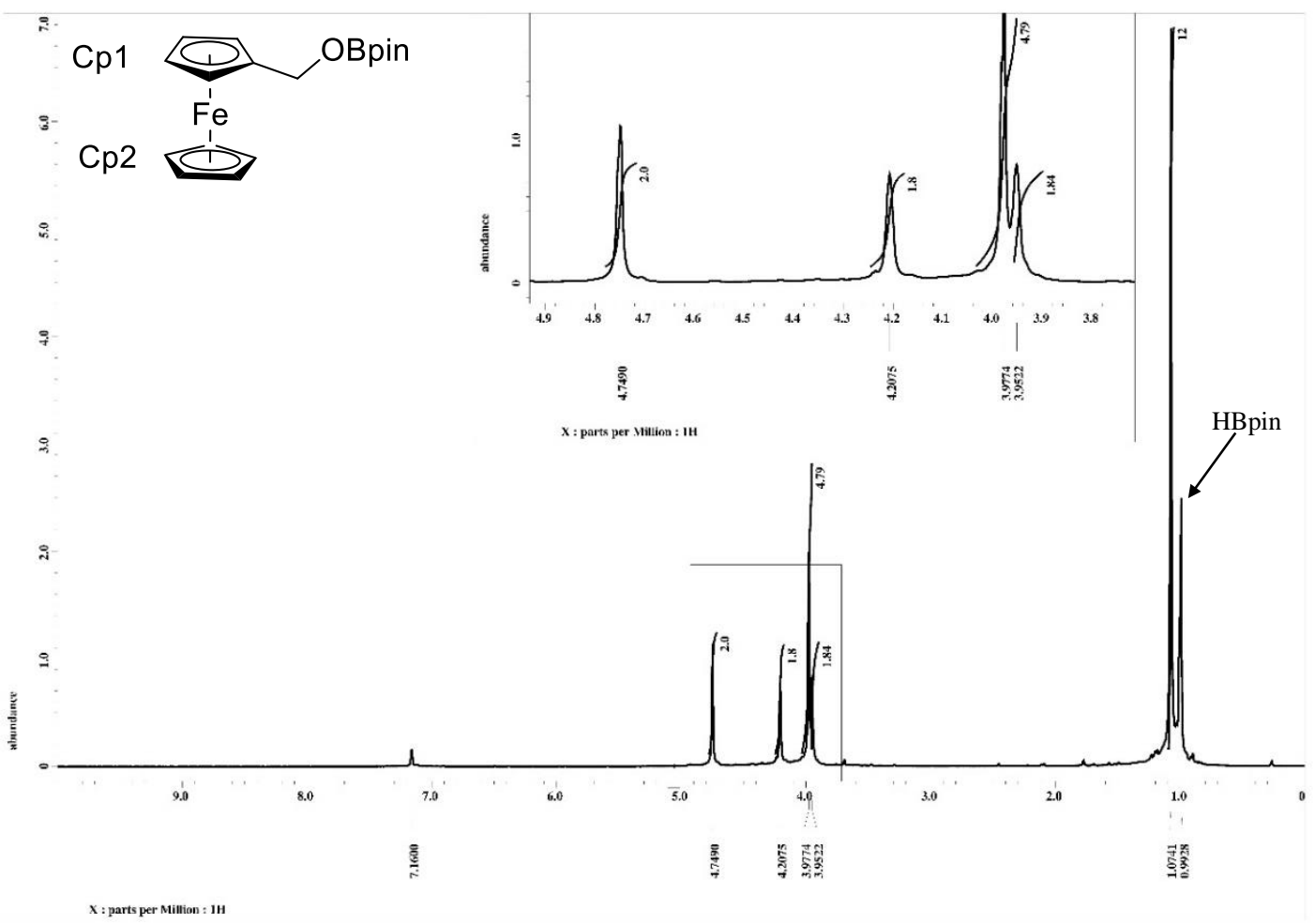

(a)

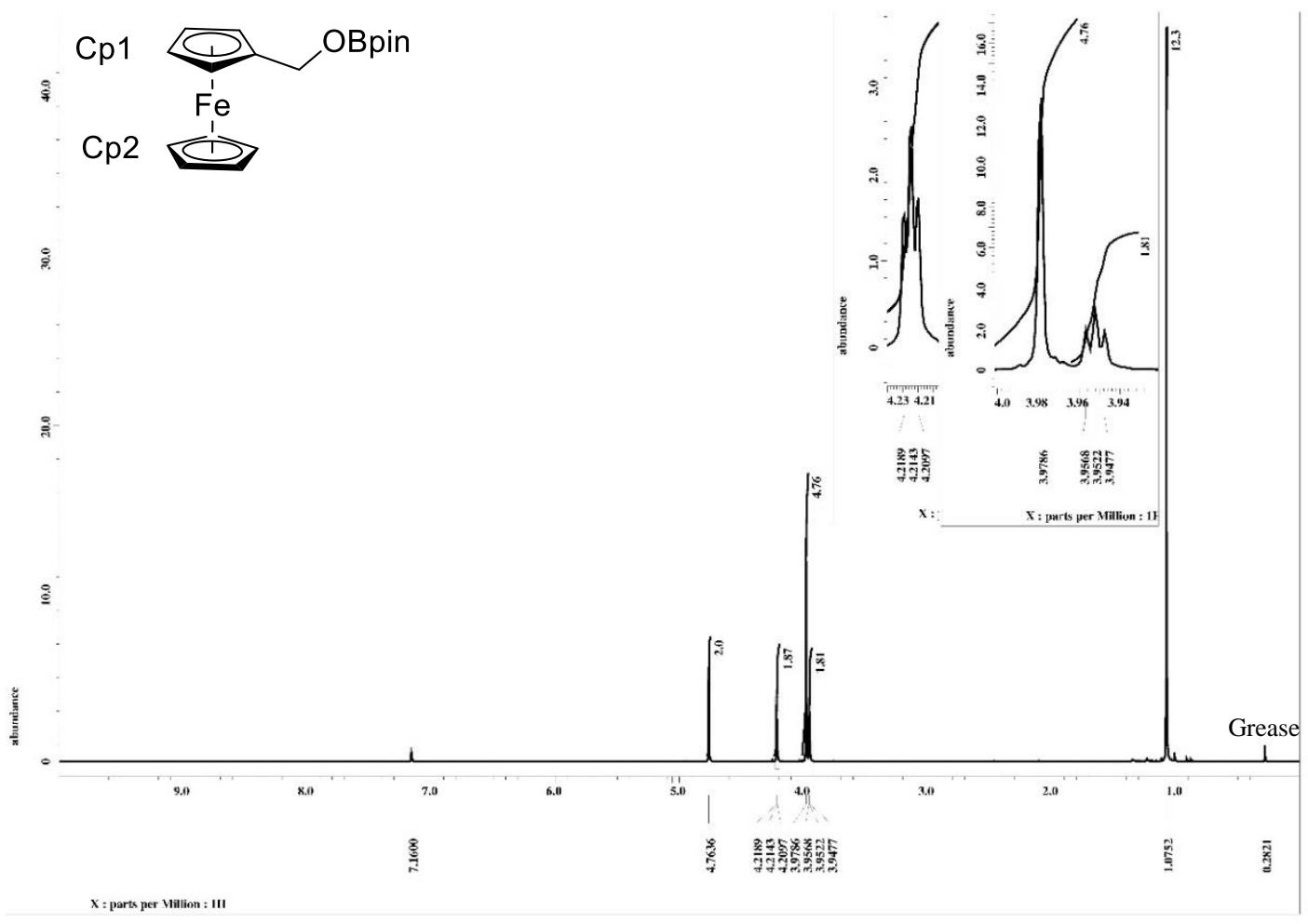

(b)

Figure S36. ${ }^{1} \mathrm{H}$ NMR spectrum of isolated compound $\mathbf{5 g}$ (in $\mathrm{C}_{6} \mathrm{D}_{6}$ ), (a) crude solution and (b) isolated compound. 


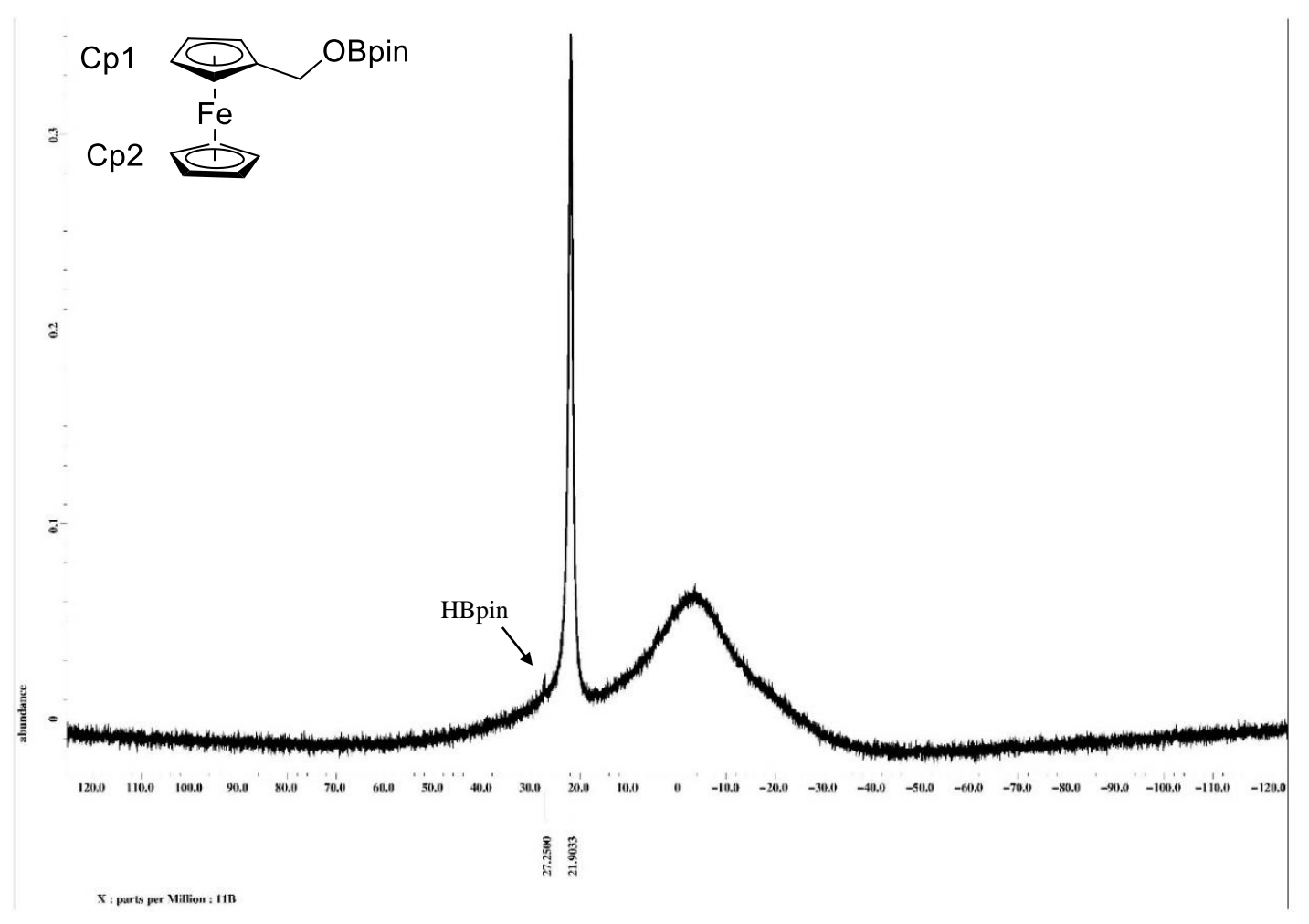

(a)

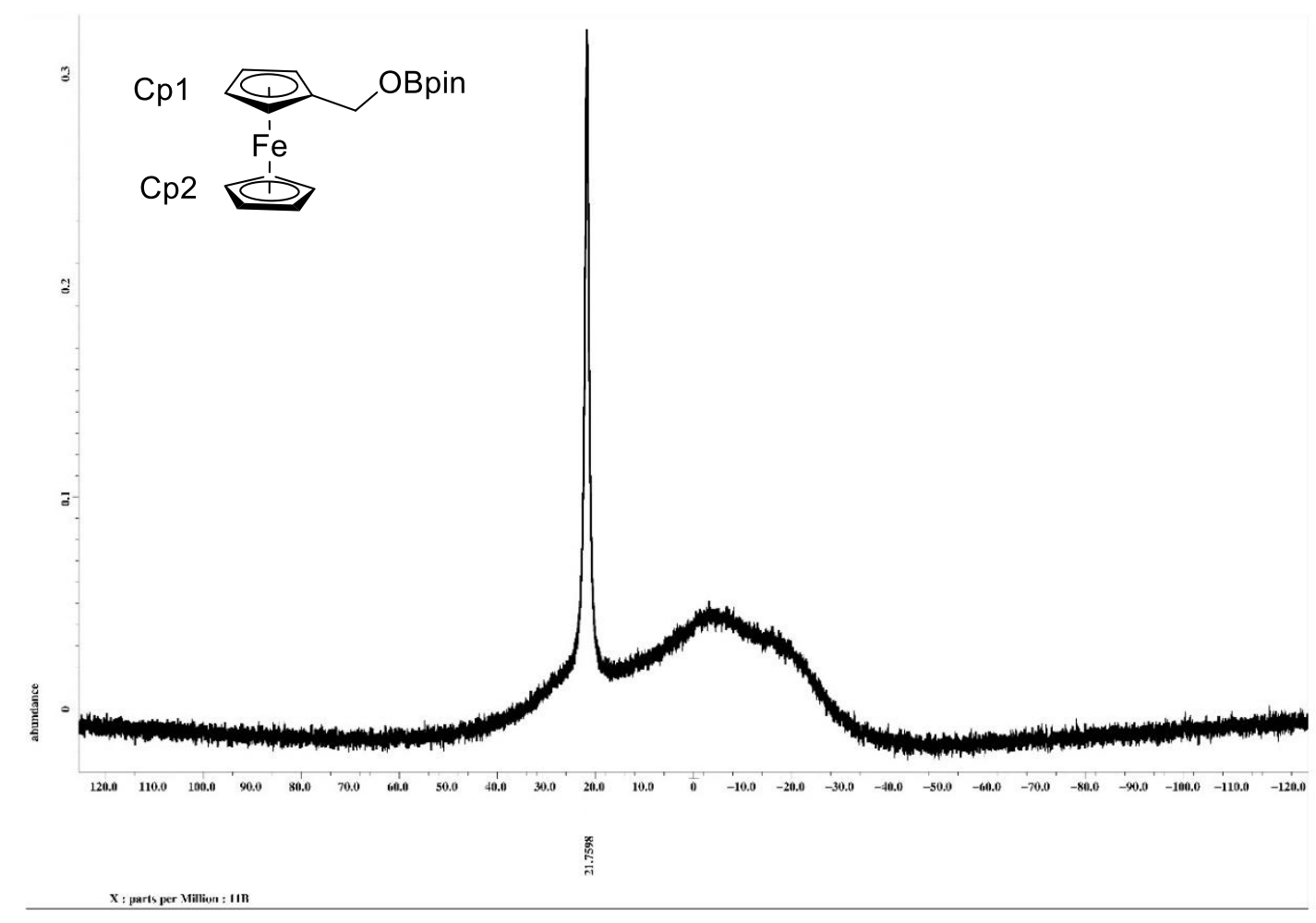

(b)

Figure S37. ${ }^{11} \mathrm{~B}\left\{{ }^{1} \mathrm{H}\right\}$ NMR spectrum of isolated compound $\mathbf{5 g}$ (in $\mathrm{C}_{6} \mathrm{D}_{6}$ ), (a) crude solution and (b) isolated compound.

Page S59 


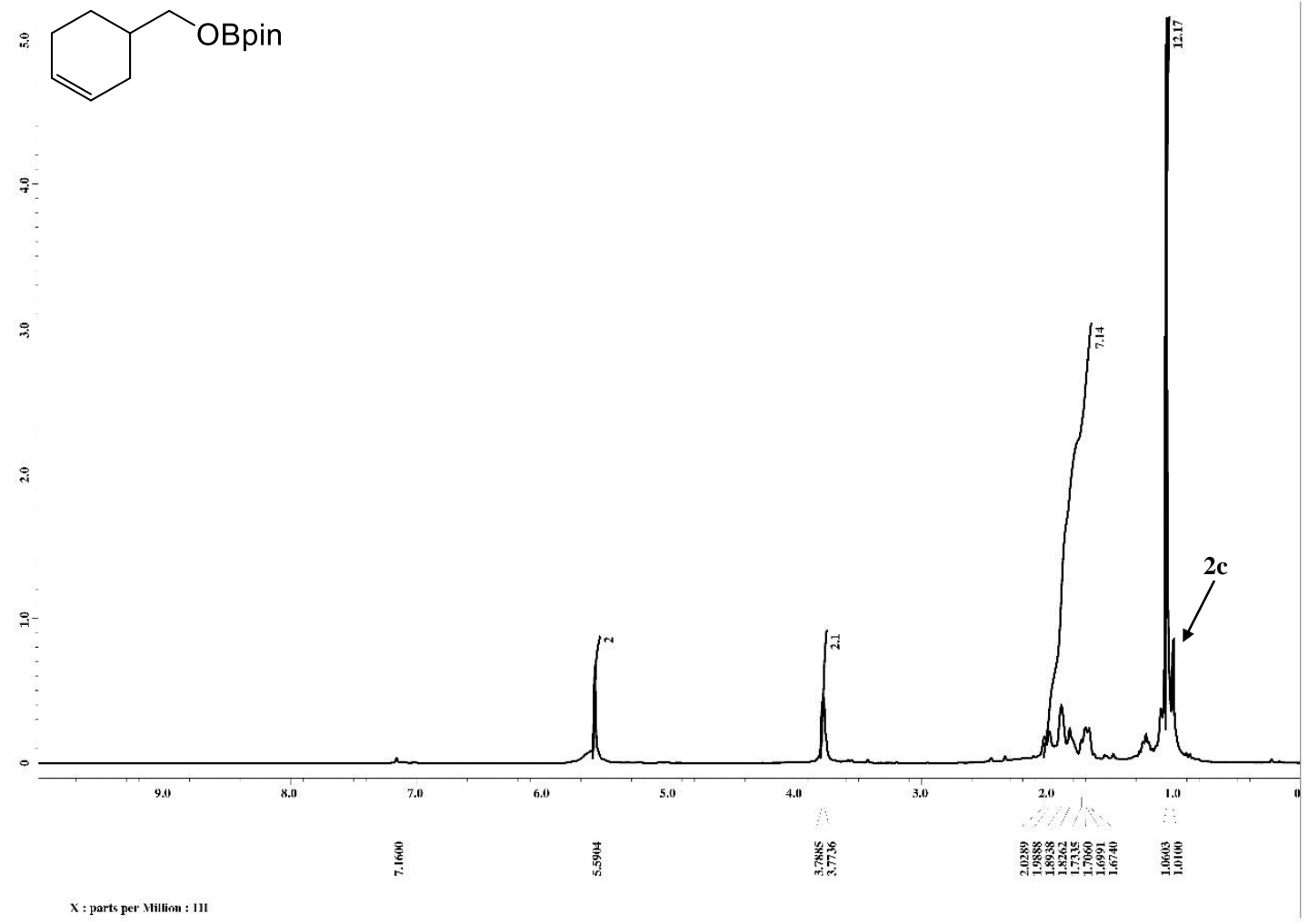

Figure S38. ${ }^{1} \mathrm{H}$ NMR spectrum of compound $5 \mathbf{h}$ (in $\mathrm{C}_{6} \mathrm{D}_{6}$ ).

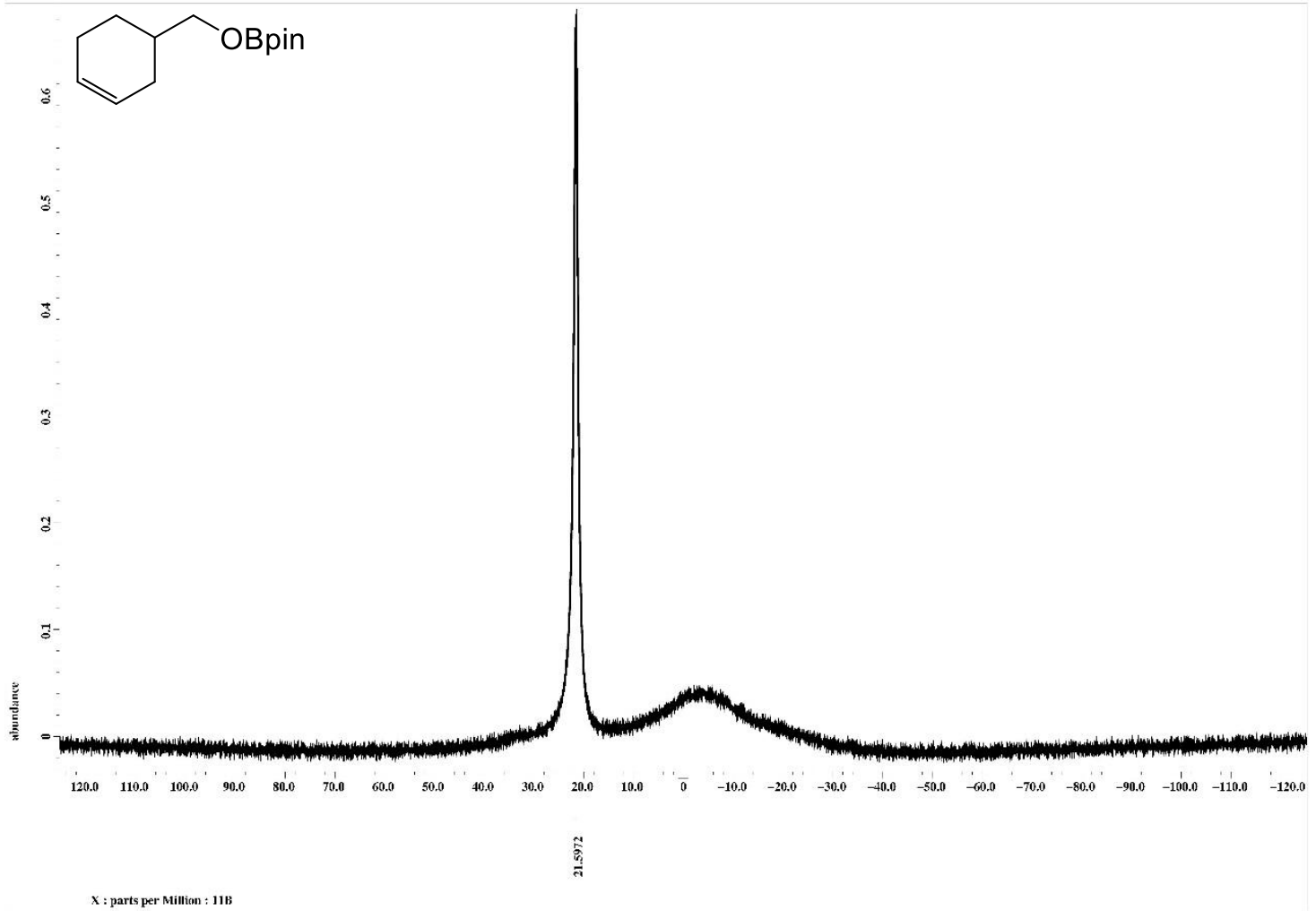

Figure S39. ${ }^{11} \mathrm{~B}\left\{{ }^{1} \mathrm{H}\right\}$ NMR spectrum of compound $\mathbf{5 h}$ (in $\mathrm{C}_{6} \mathrm{D}_{6}$ ). 


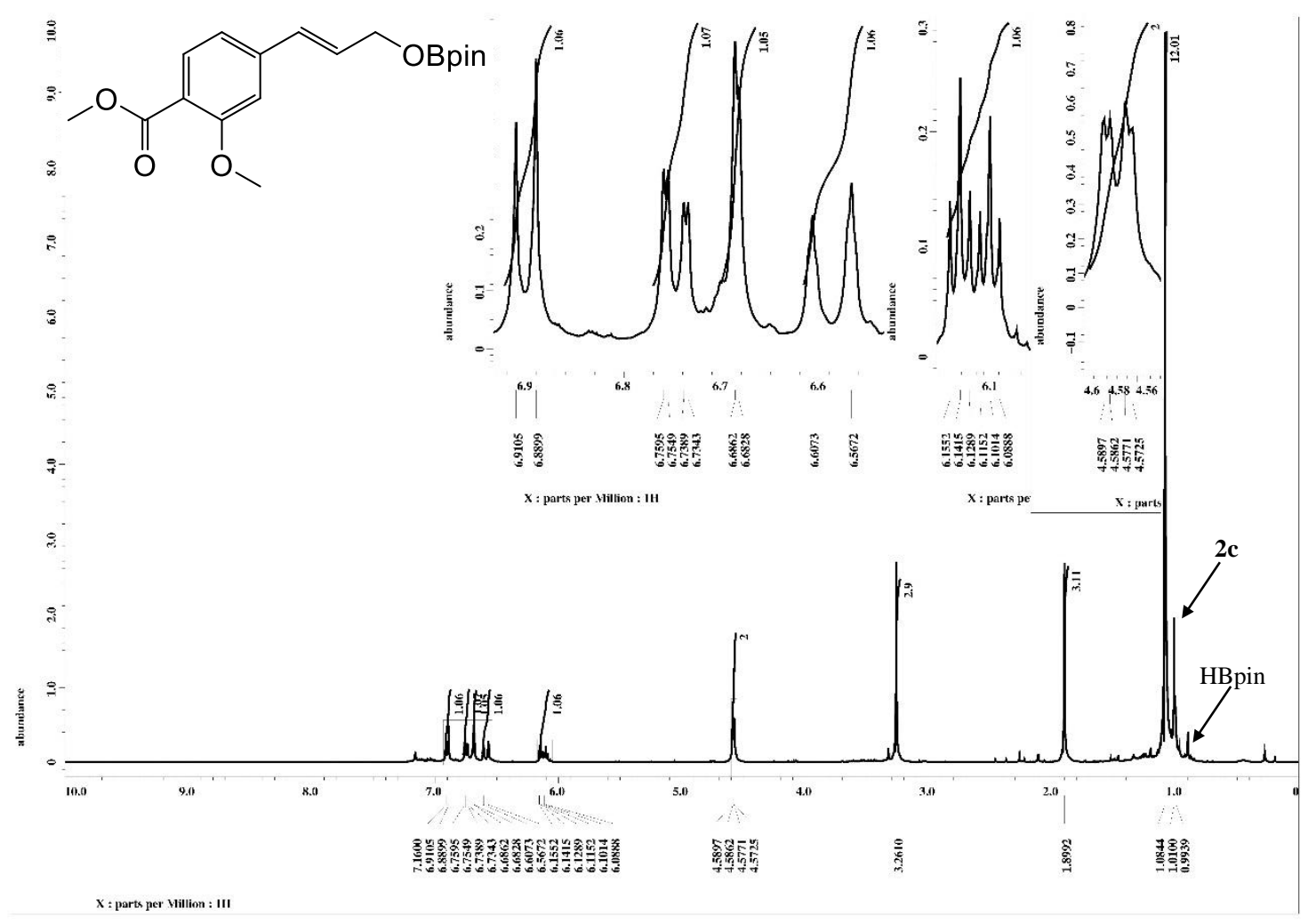

Figure S40. ${ }^{1} \mathrm{H}$ NMR spectrum of compound $5 \mathbf{j}$ (in $\mathrm{C}_{6} \mathrm{D}_{6}$ ).

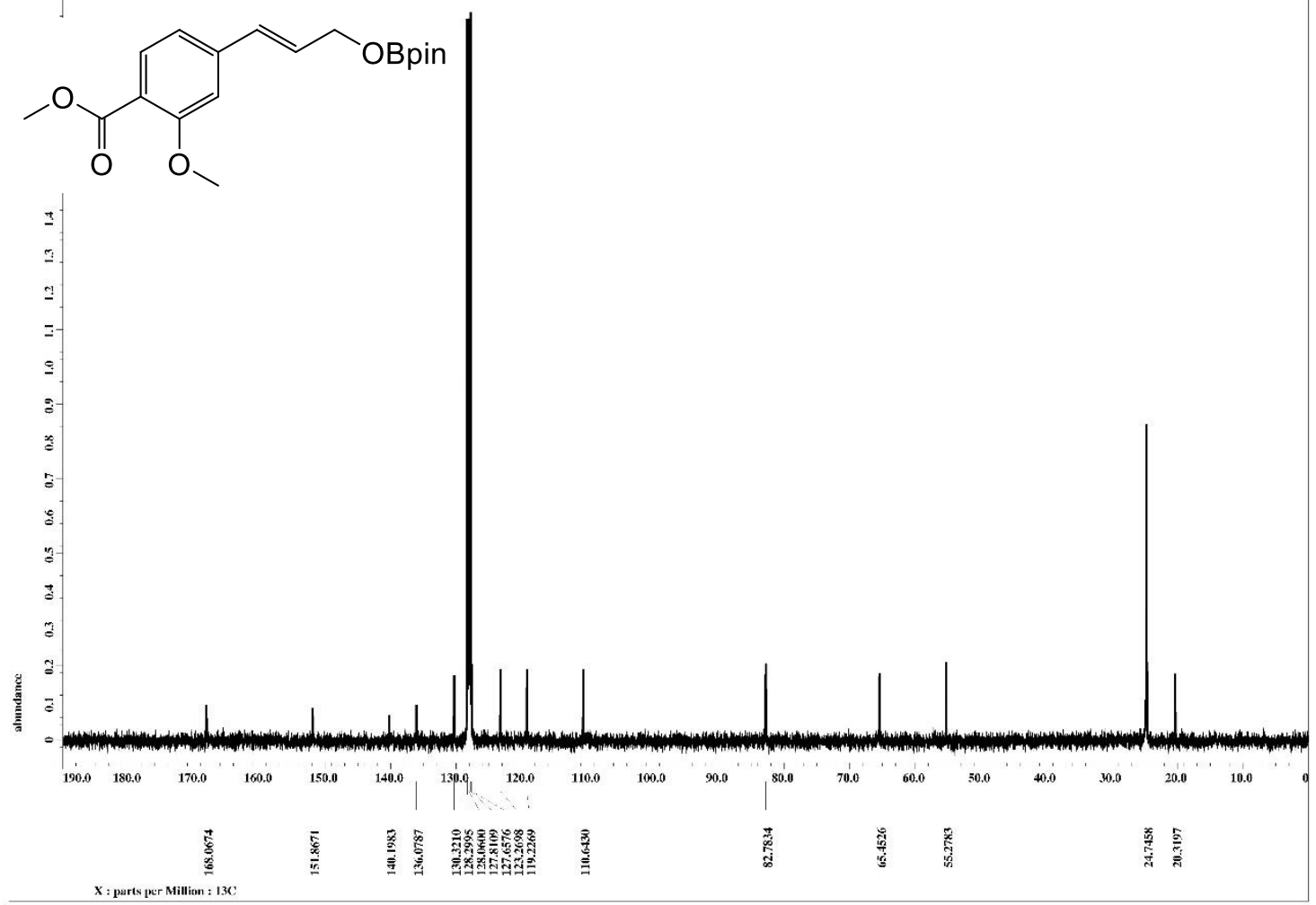

Figure S41. ${ }^{13} \mathrm{C}$ NMR spectrum of compound $\mathbf{5 j}$ (in $\mathrm{C}_{6} \mathrm{D}_{6}$ ). 


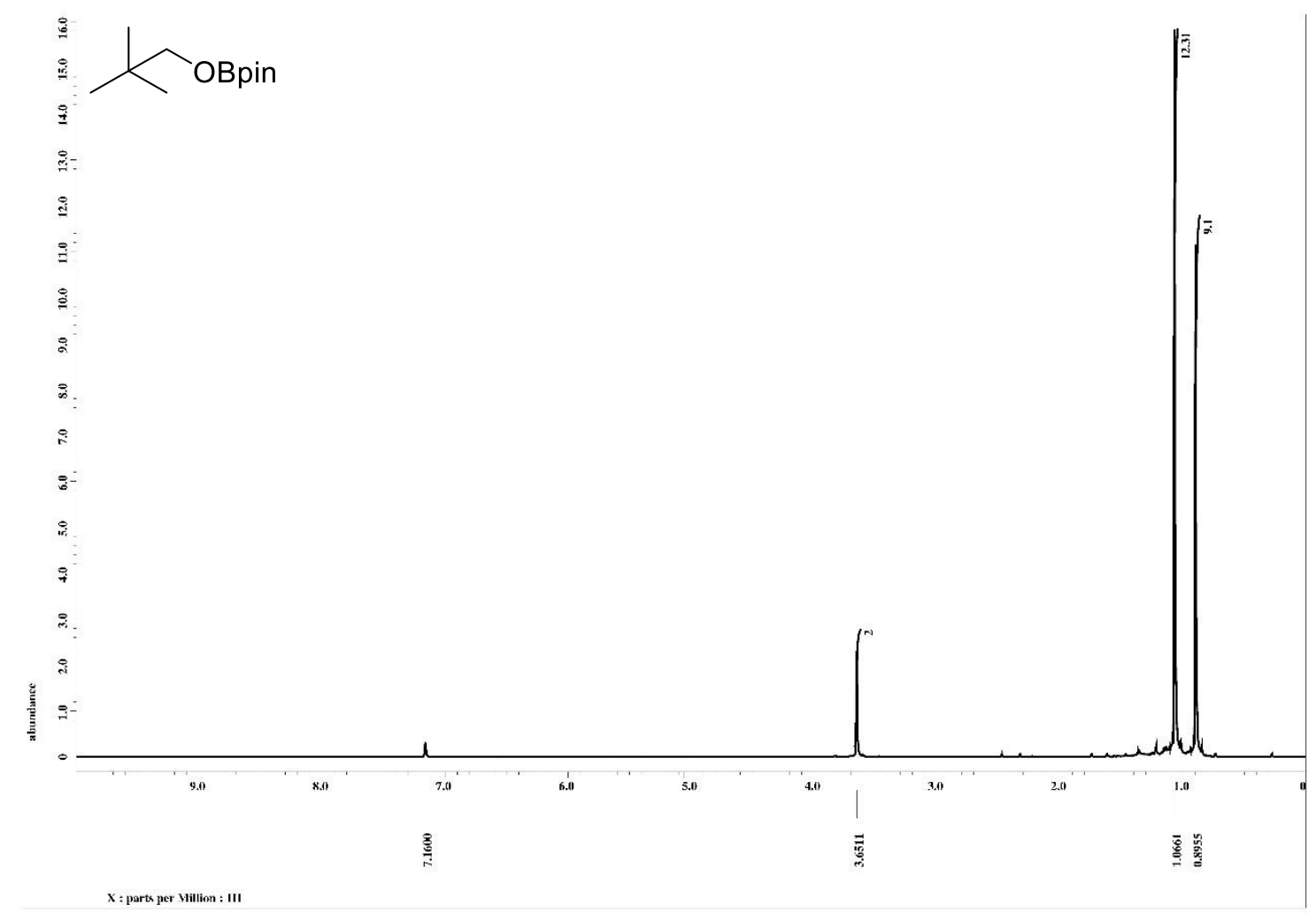

Figure S42. ${ }^{1} \mathrm{H}$ NMR spectrum of compound $5 \mathbf{k}$ (in $\mathrm{C}_{6} \mathrm{D}_{6}$ ).

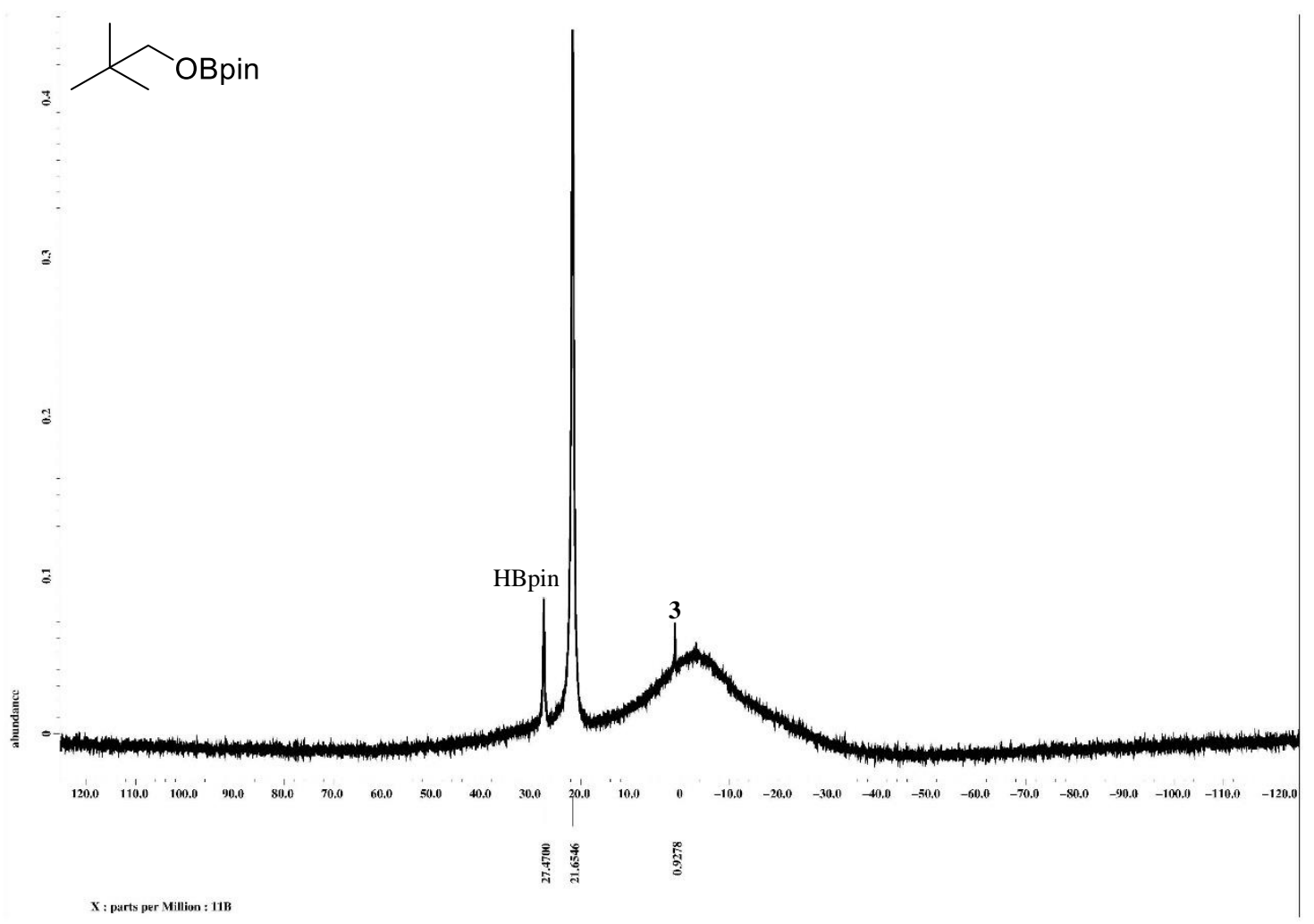

Figure S43. ${ }^{11} \mathrm{~B}\left\{{ }^{1} \mathrm{H}\right\}$ NMR spectrum of compound $\mathbf{5 k}$ (in $\mathrm{C}_{6} \mathrm{D}_{6}$ ). 


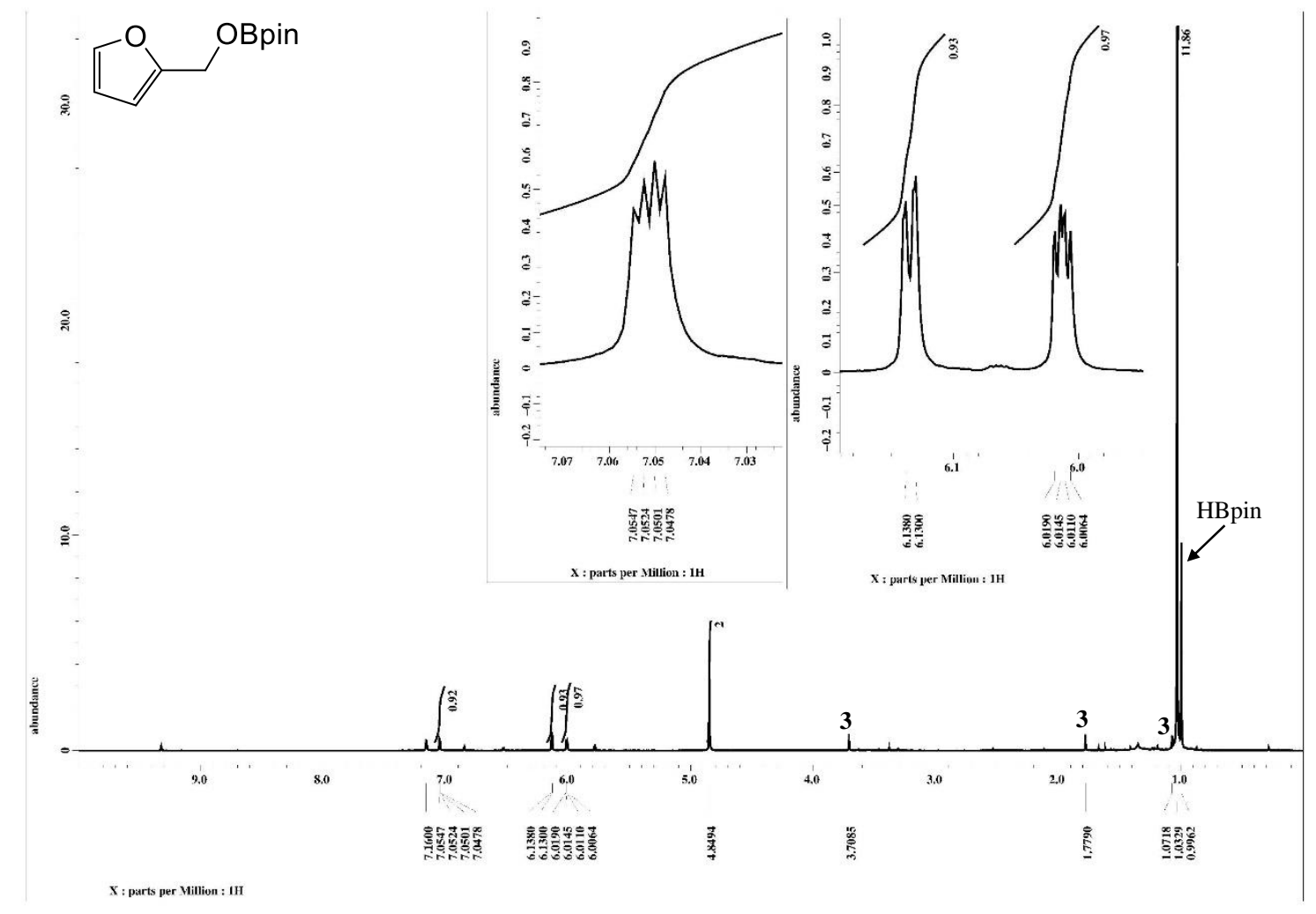

Figure S44. ${ }^{1} \mathrm{H}$ NMR spectrum of compound $\mathbf{5 l}$ (in $\mathrm{C}_{6} \mathrm{D}_{6}$ ).

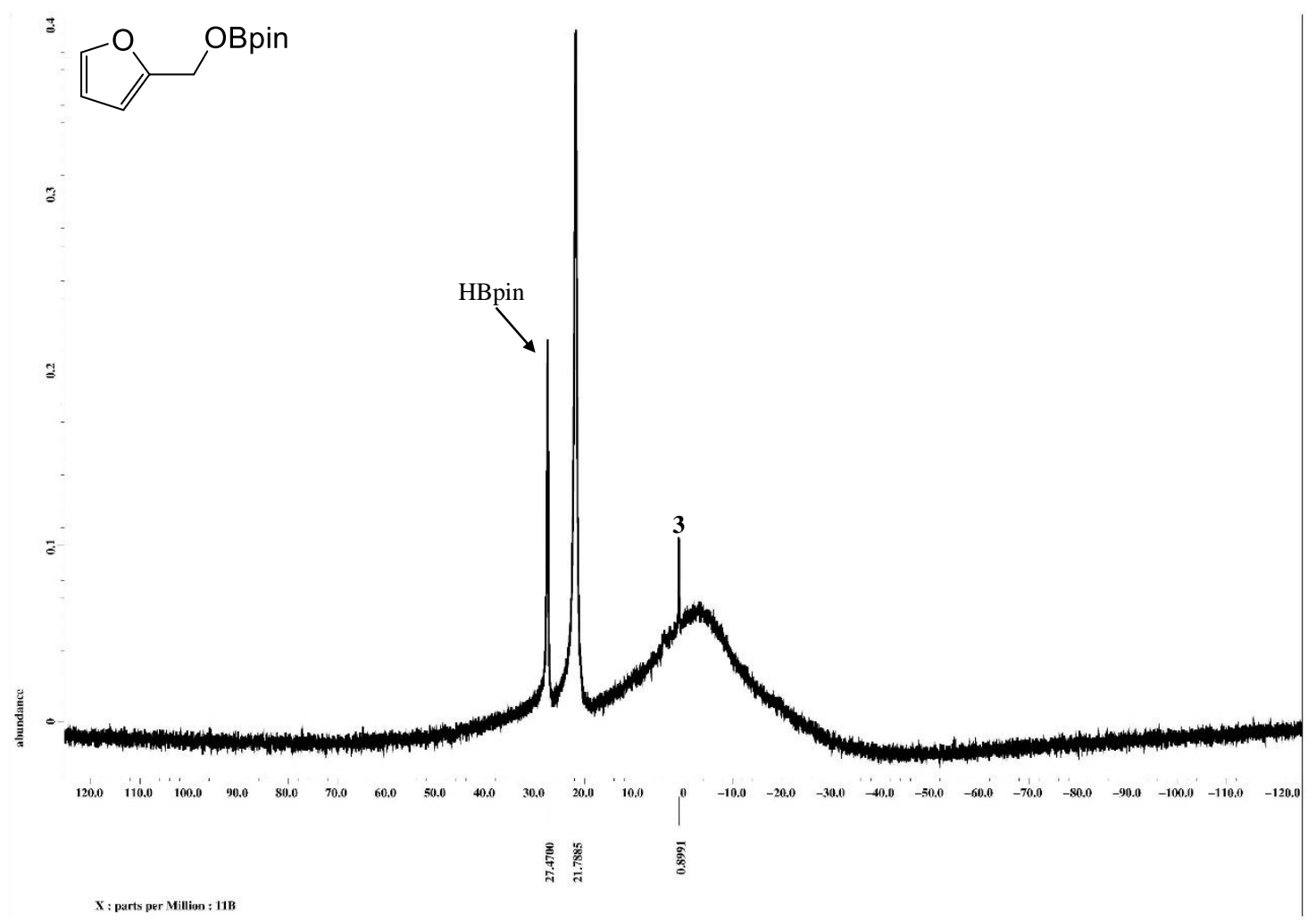

Figure S45. ${ }^{11} \mathrm{~B}\left\{{ }^{1} \mathrm{H}\right\}$ NMR spectrum of compound $\mathbf{5 l}$ (in $\mathrm{C}_{6} \mathrm{D}_{6}$ ). 


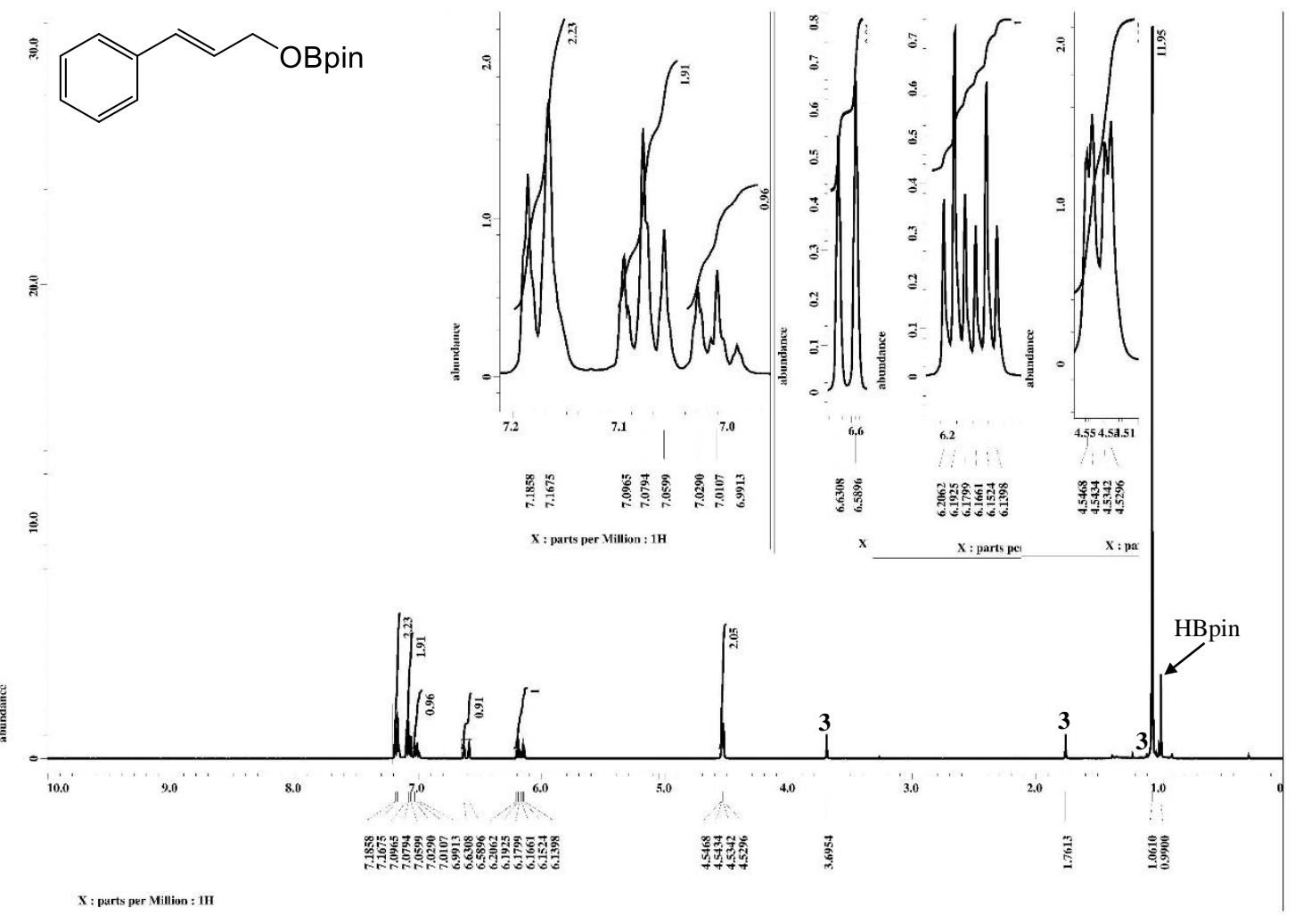

Figure S46. ${ }^{1} \mathrm{H}$ NMR spectrum of compound $\mathbf{5 m}$ (in $\mathrm{C}_{6} \mathrm{D}_{6}$ ).

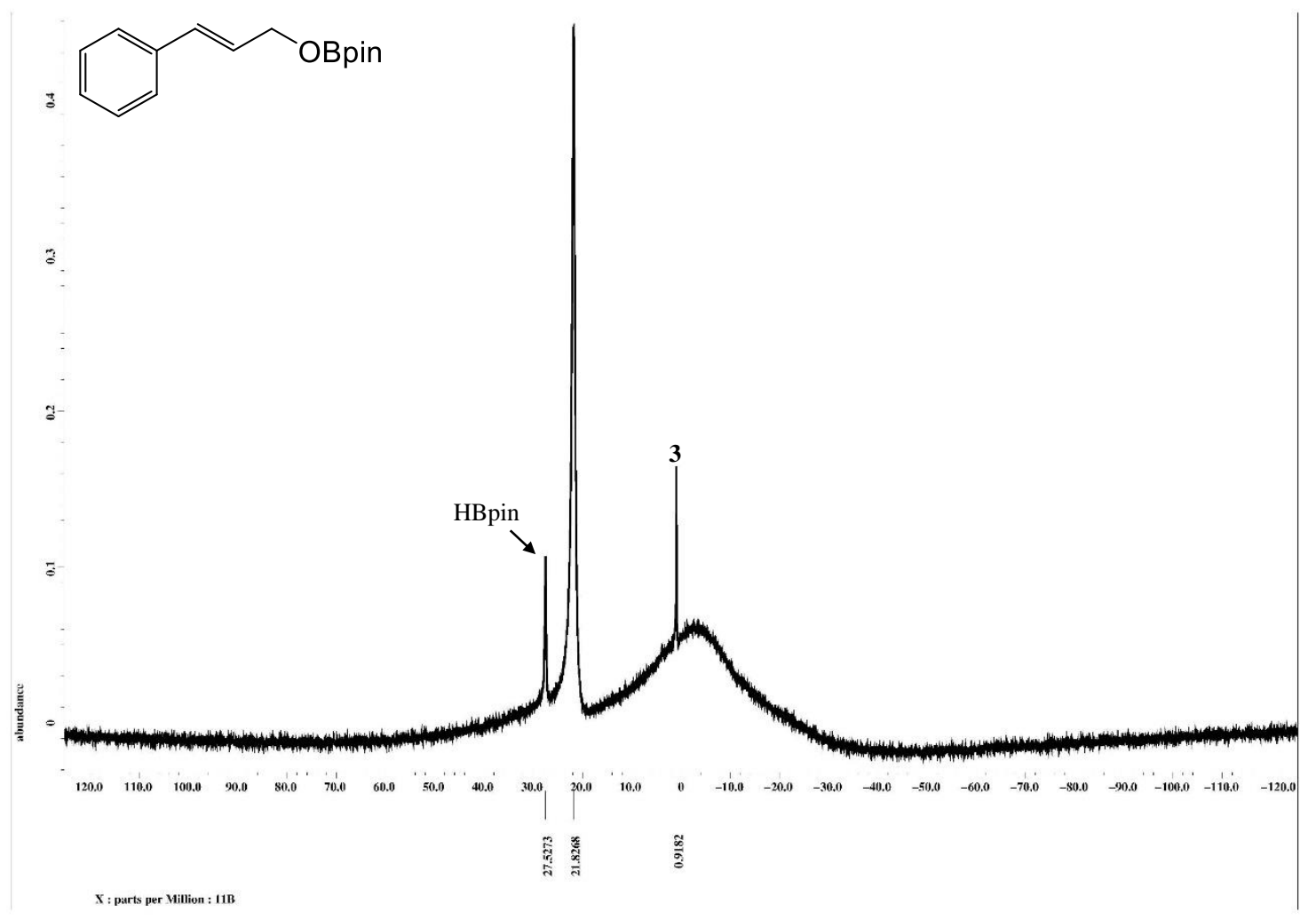

Figure S47. ${ }^{11} \mathrm{~B}\left\{{ }^{1} \mathrm{H}\right\}$ NMR spectrum of compound $\mathbf{5 m}$ (in $\mathrm{C}_{6} \mathrm{D}_{6}$ ). 


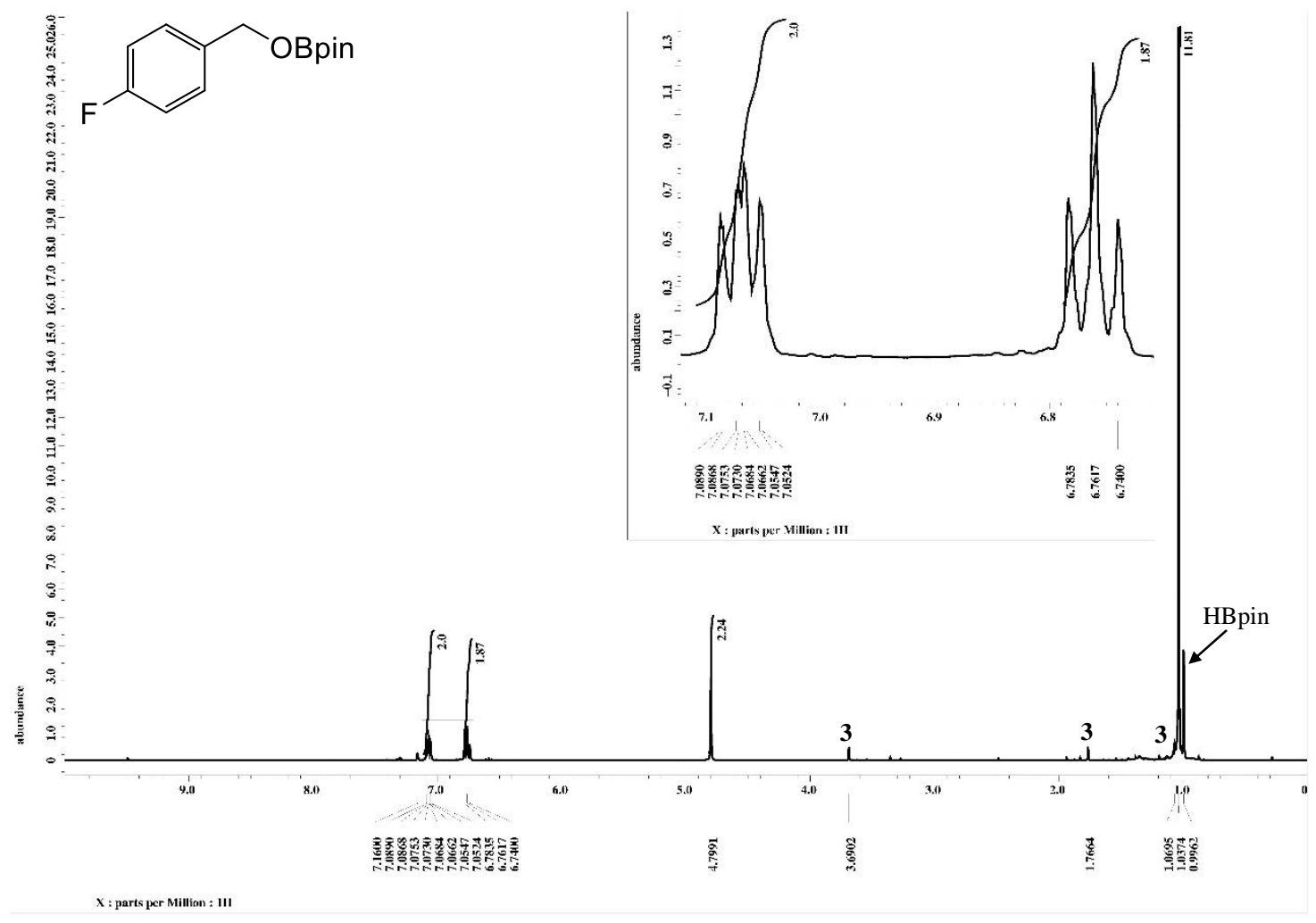

Figure S48. ${ }^{1} \mathrm{H}$ NMR spectrum of compound 5 n (in $\mathrm{C}_{6} \mathrm{D}_{6}$ ).

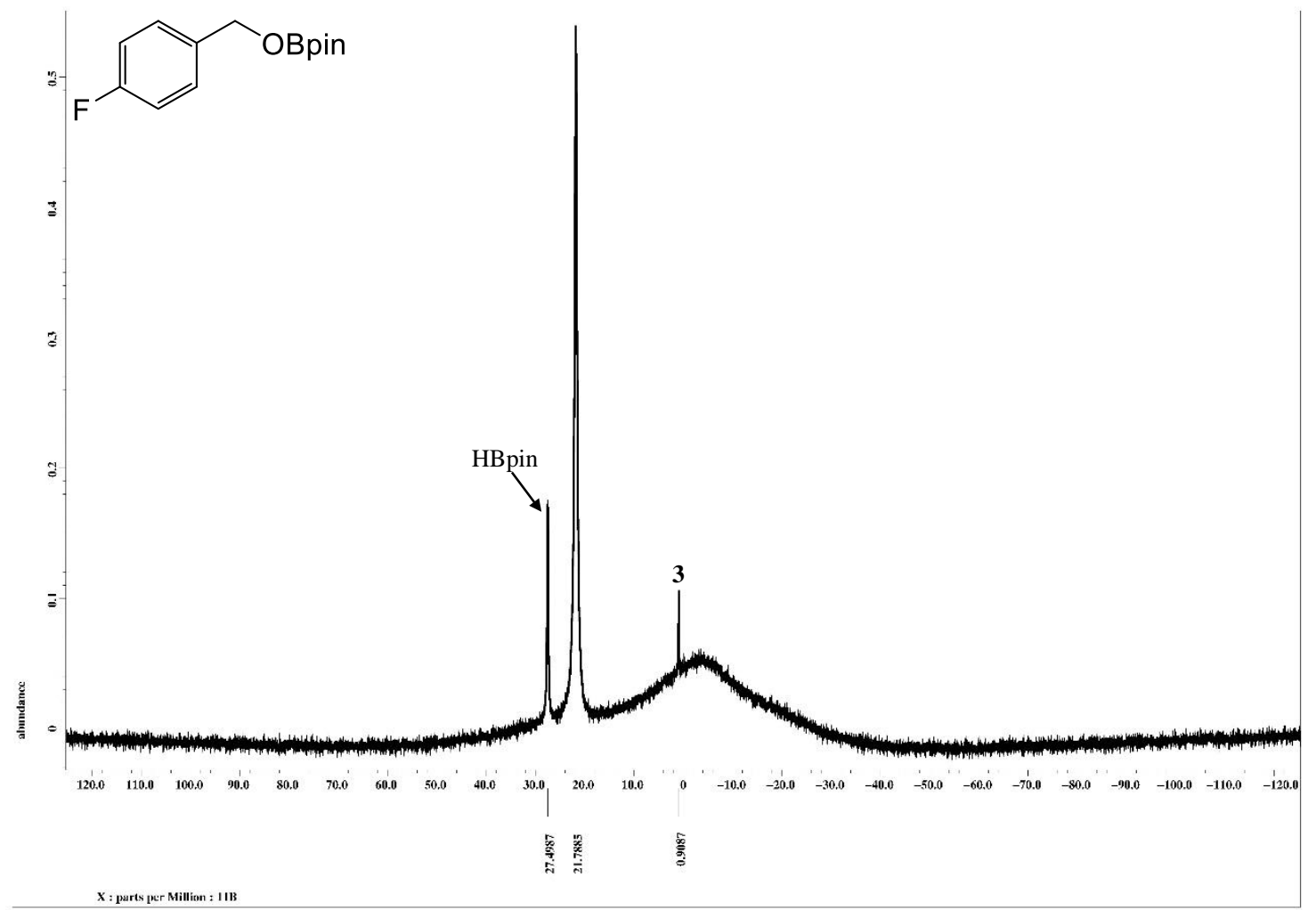

Figure S49. ${ }^{11} \mathrm{~B}\left\{{ }^{1} \mathrm{H}\right\}$ NMR spectrum of compound $\mathbf{5 n}$ (in $\mathrm{C}_{6} \mathrm{D}_{6}$ ). 


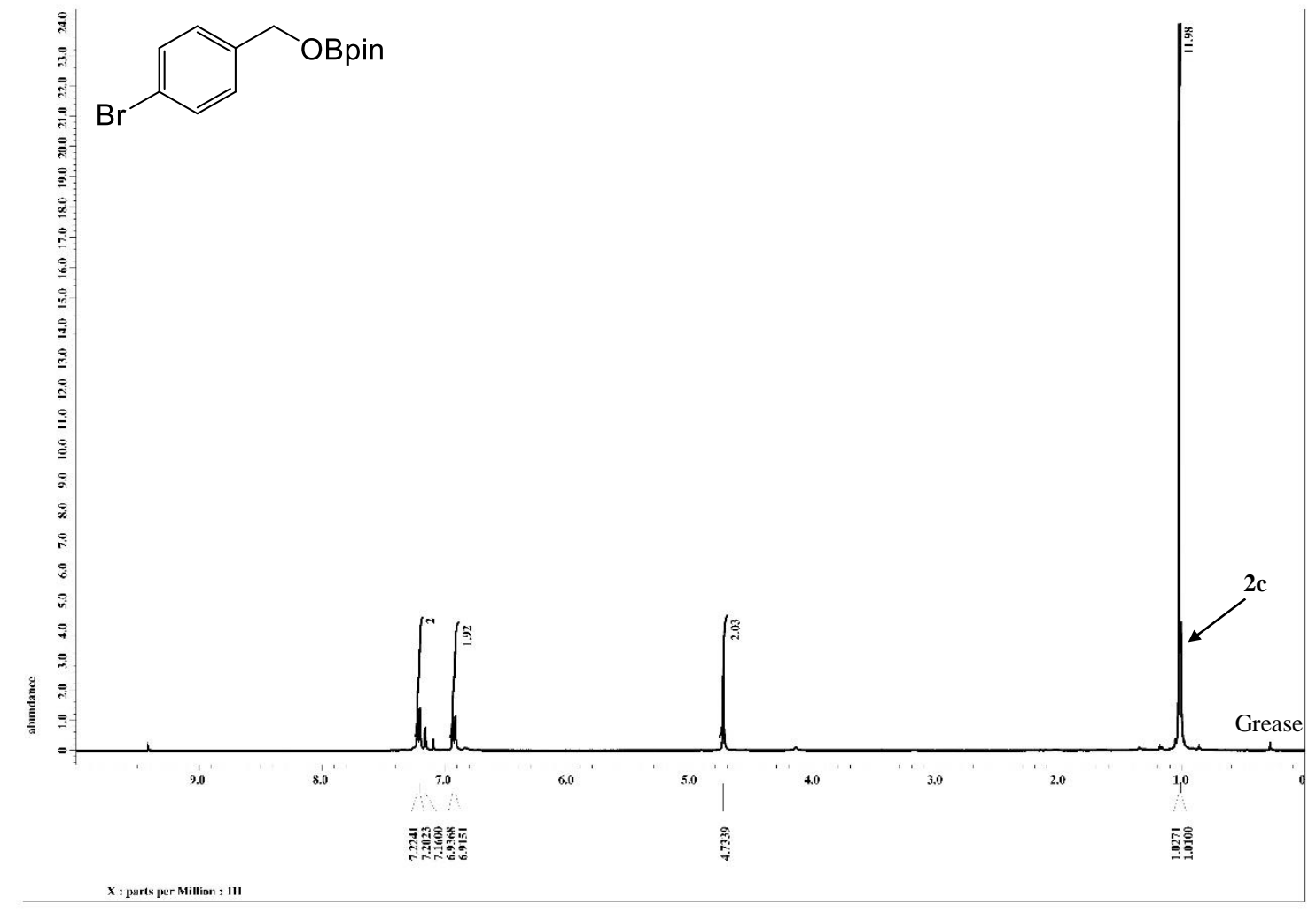

Figure S50. ${ }^{1} \mathrm{H}$ NMR spectrum of compound $5 \mathbf{p}$ (in $\mathrm{C}_{6} \mathrm{D}_{6}$ ).

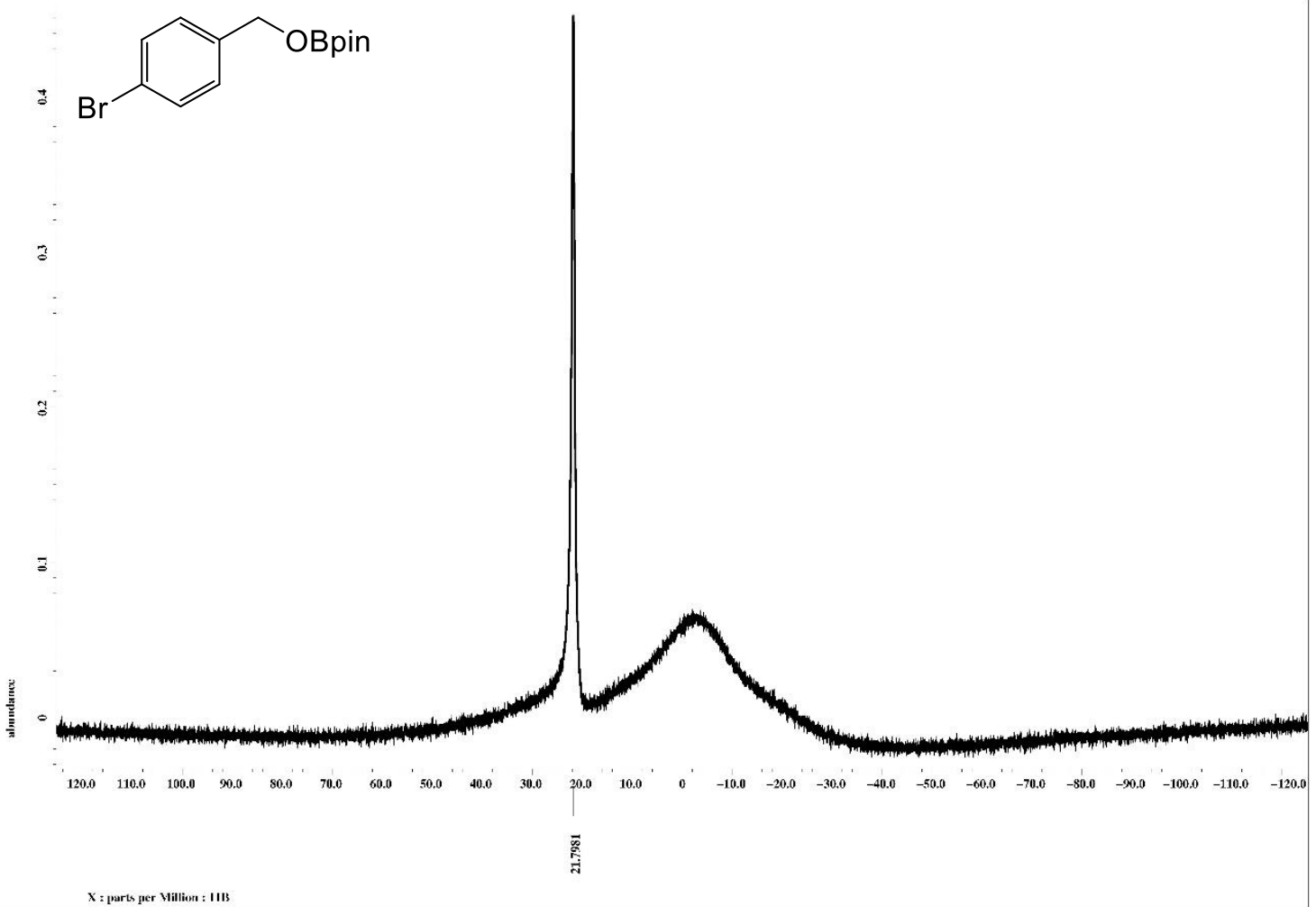

Figure S51. ${ }^{11} \mathrm{~B}\left\{{ }^{1} \mathrm{H}\right\}$ NMR spectrum of compound $\mathbf{5 p}$ (in $\mathrm{C}_{6} \mathrm{D}_{6}$ ). 


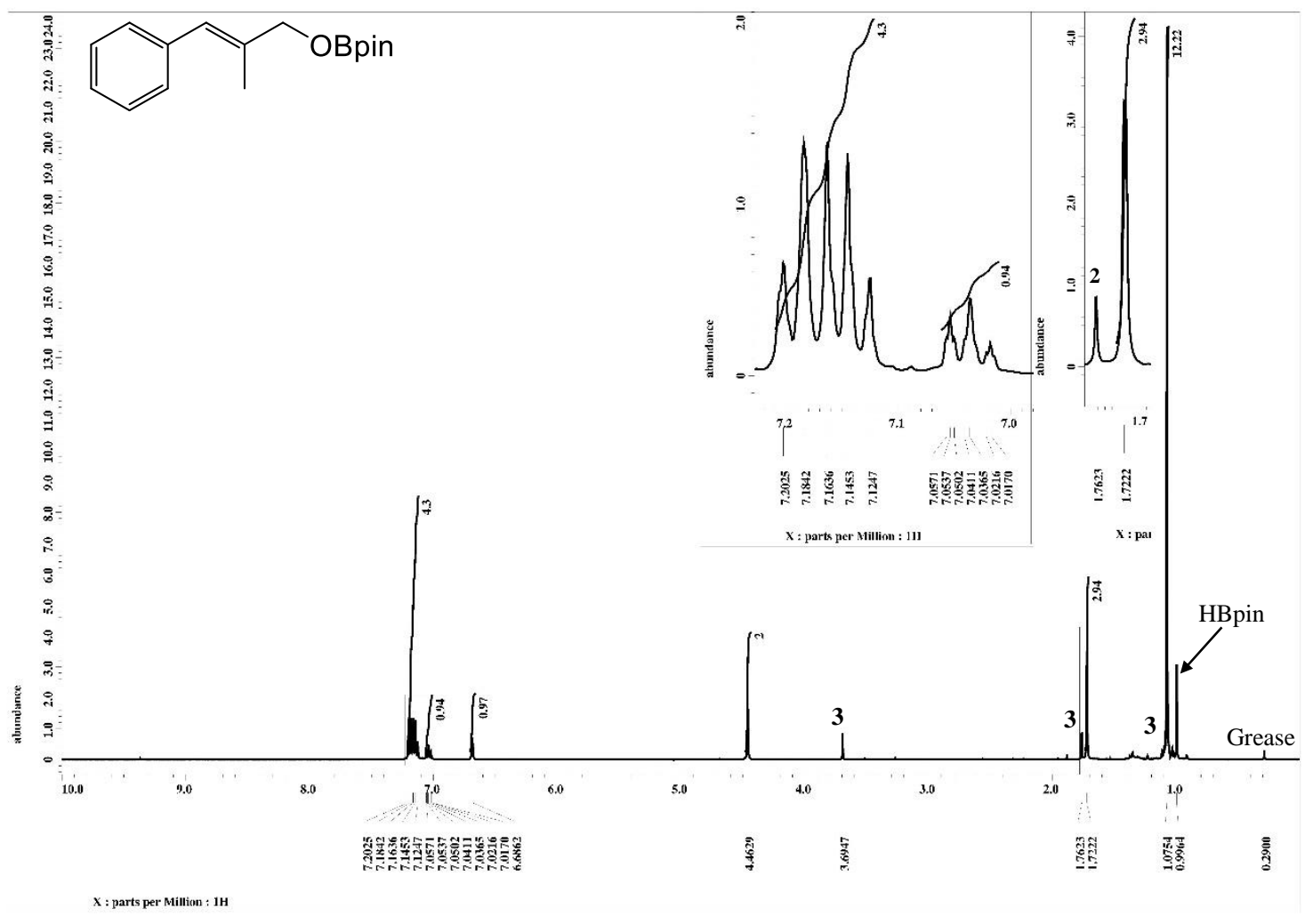

Figure S52. ${ }^{1} \mathrm{H}$ NMR spectrum of compound $\mathbf{5 q}$ (in $\mathrm{C}_{6} \mathrm{D}_{6}$ ).

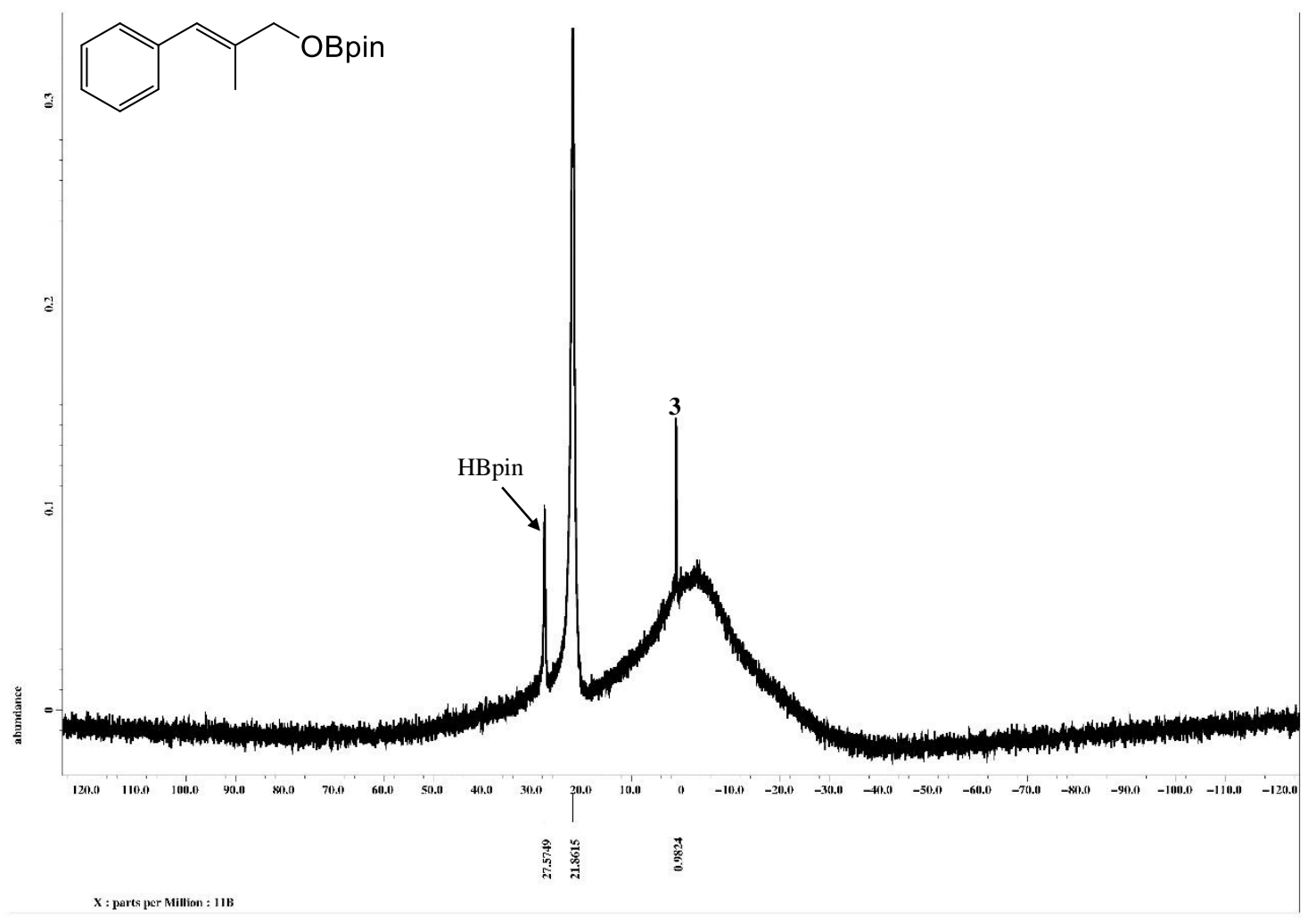

Figure S53. ${ }^{11} \mathrm{~B}\left\{{ }^{1} \mathrm{H}\right\}$ NMR spectrum of compound $\mathbf{5 q}\left(\right.$ in $\mathrm{C}_{6} \mathrm{D}_{6}$ ). 


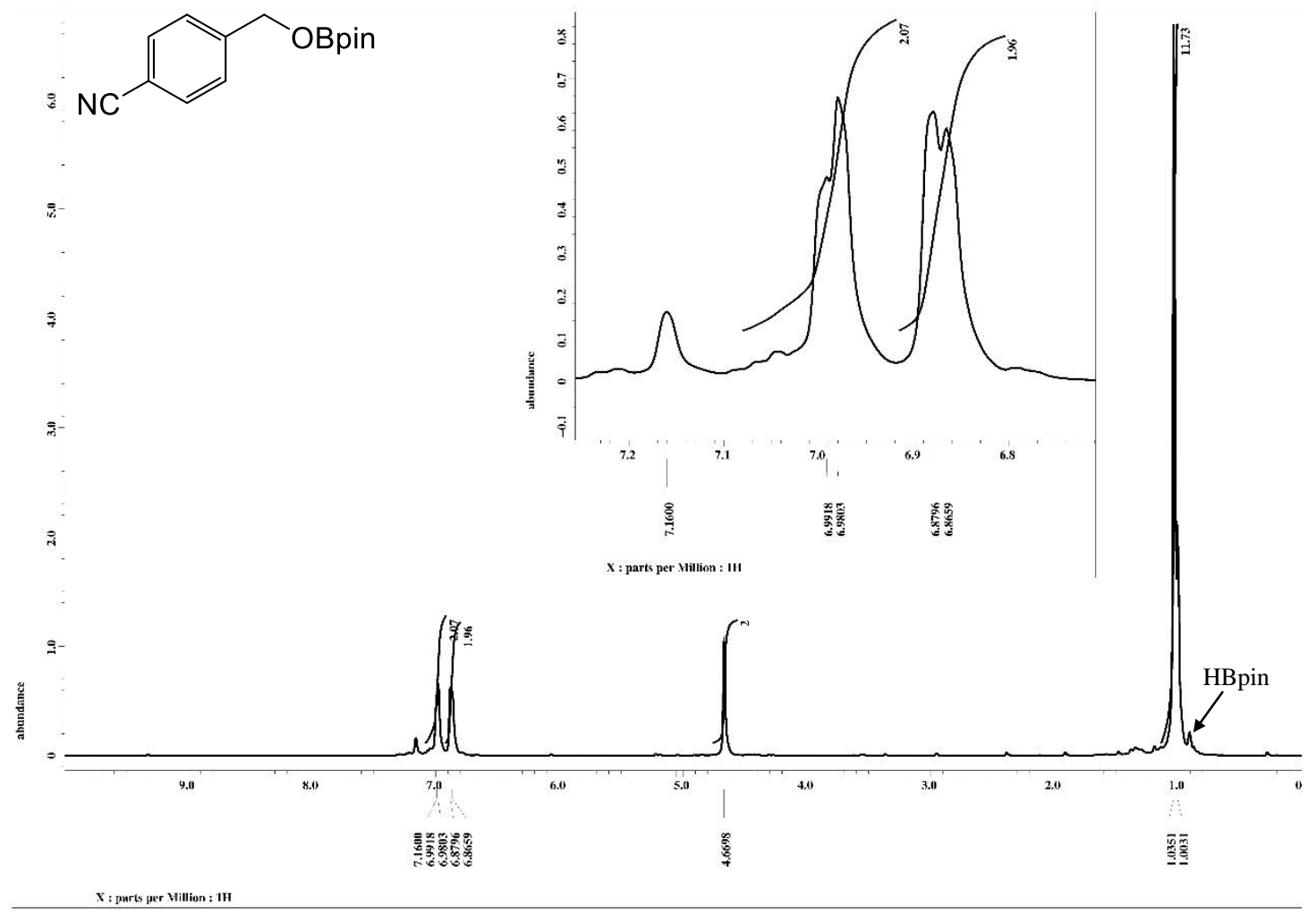

Figure S54. ${ }^{1} \mathrm{H}$ NMR spectrum of compound $5 \mathbf{r}$ (in $\mathrm{C}_{6} \mathrm{D}_{6}$ ).

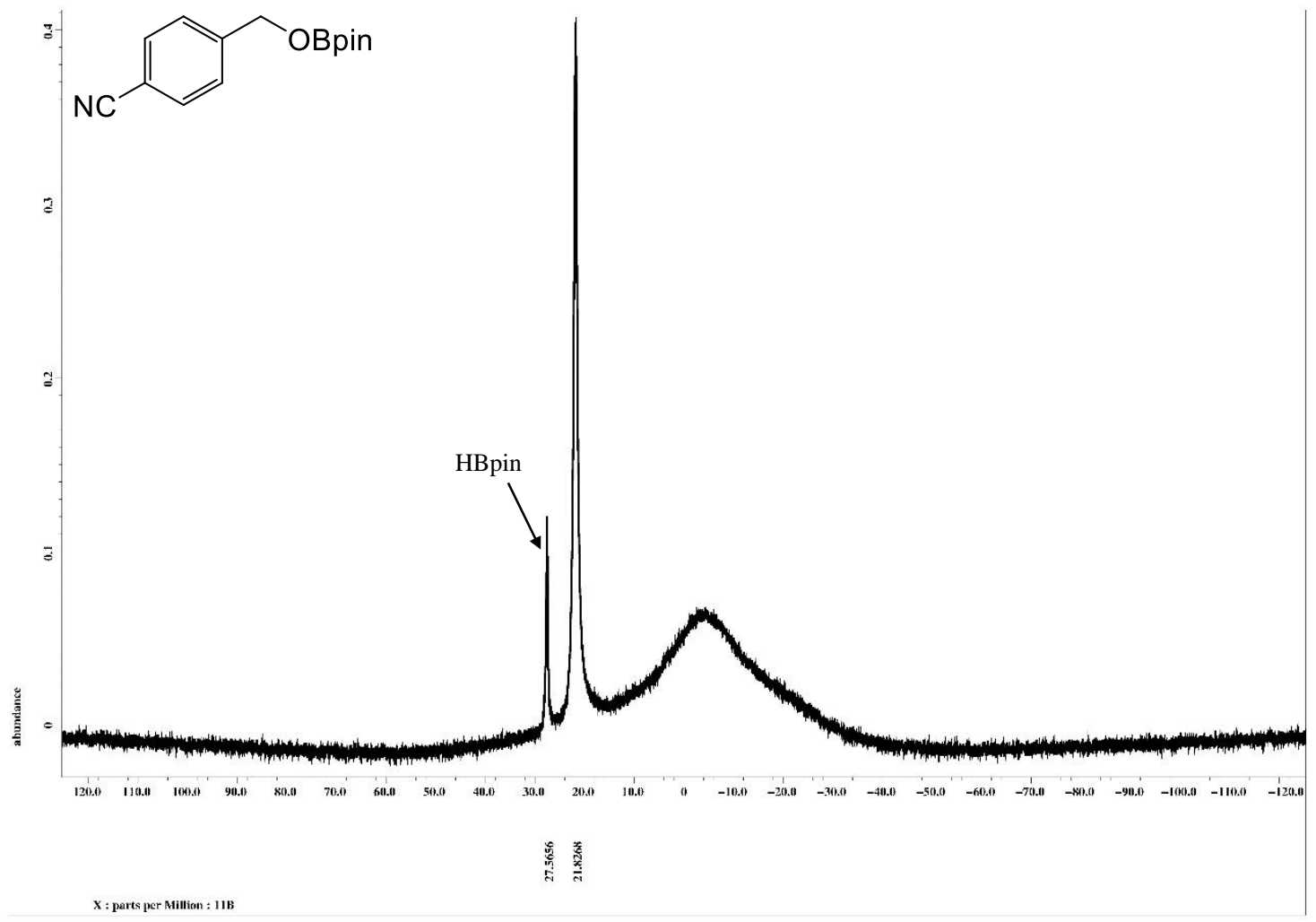

Figure S55. ${ }^{11} \mathrm{~B}\left\{{ }^{1} \mathrm{H}\right\}$ NMR spectrum of compound $\mathbf{5 r}\left(\right.$ in $\mathrm{C}_{6} \mathrm{D}_{6}$ ). 


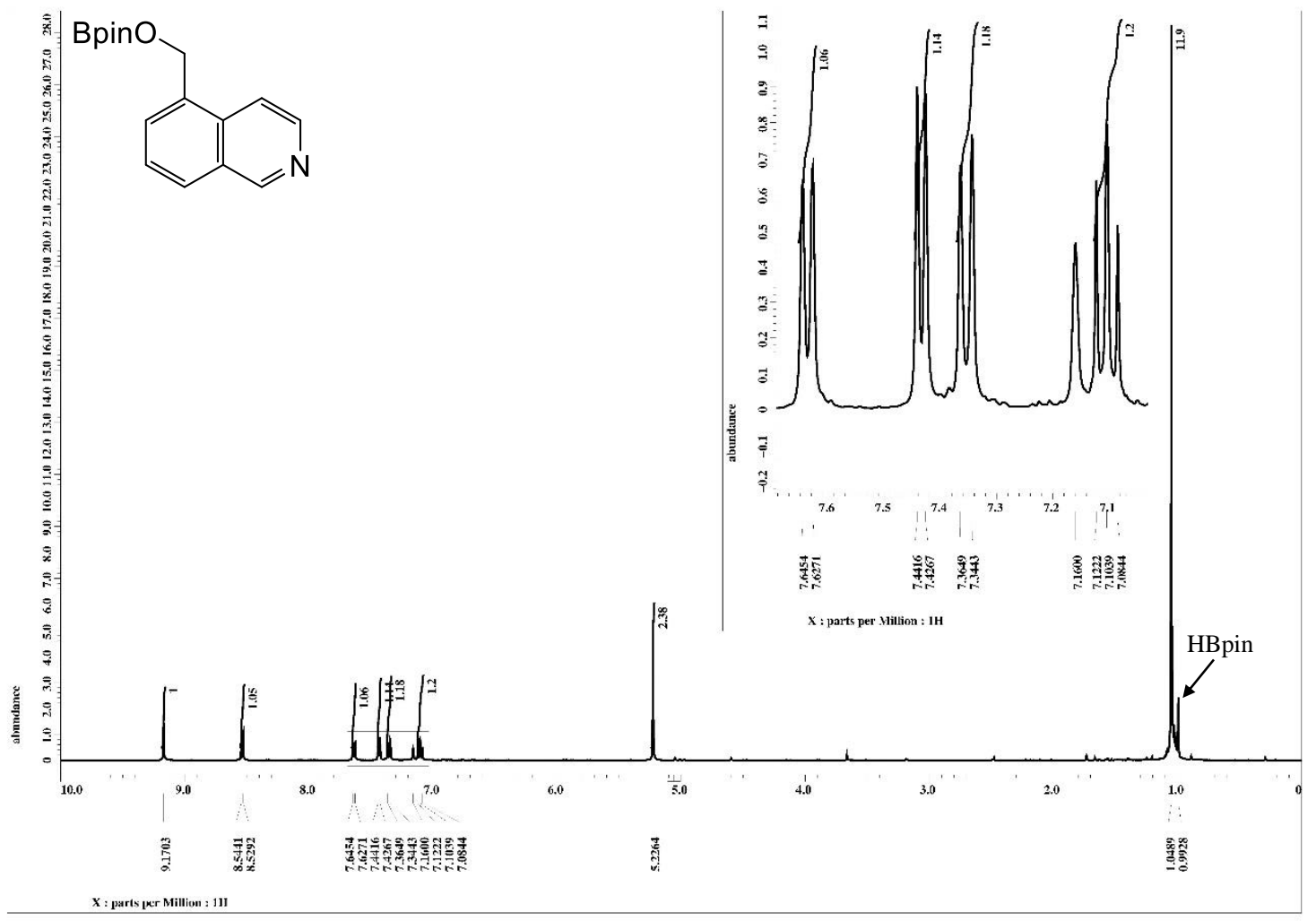

Figure S56. ${ }^{1} \mathrm{H}$ NMR spectrum of compound 5 s (in $\mathrm{C}_{6} \mathrm{D}_{6}$ ).

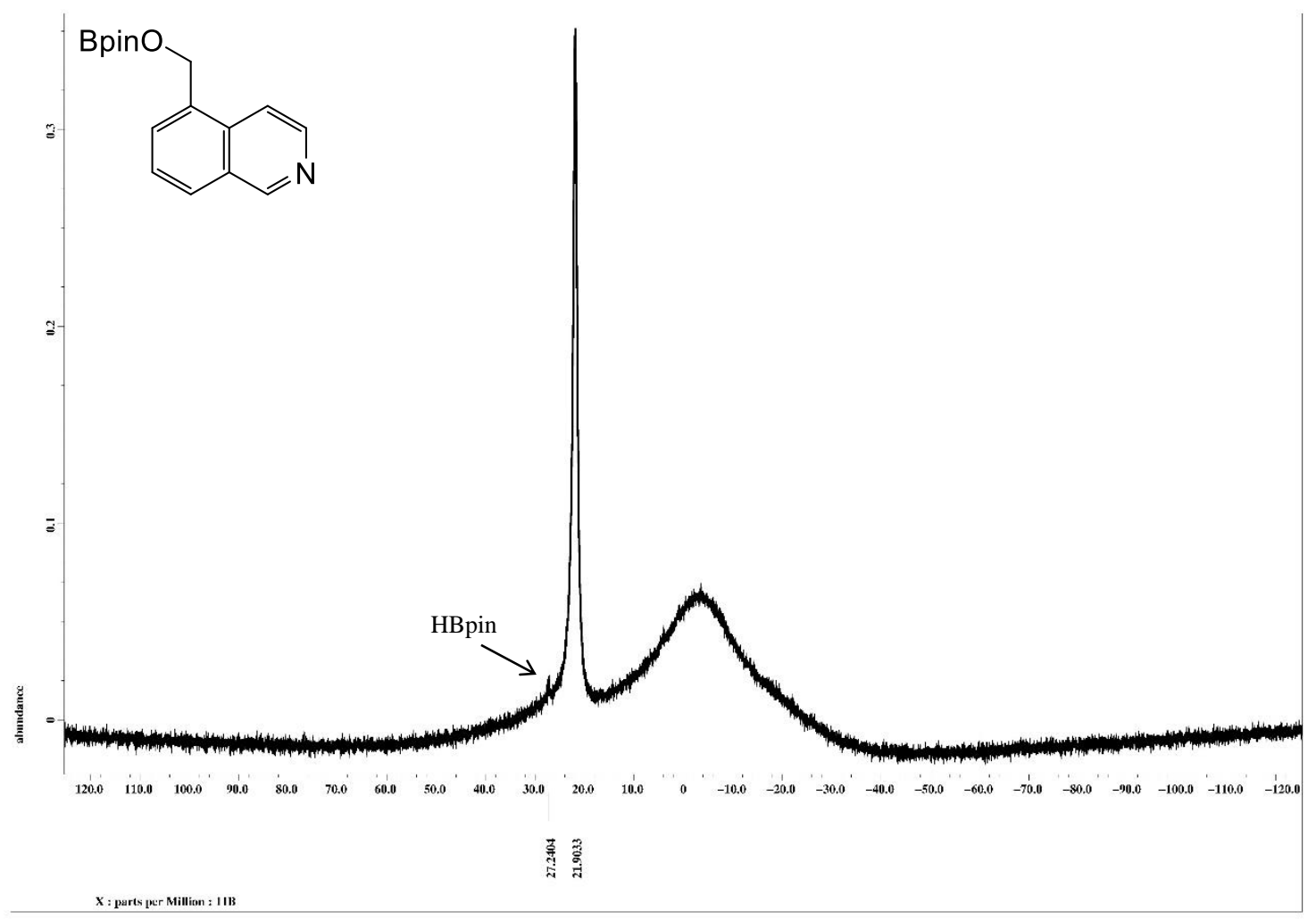

Figure S57. ${ }^{11} \mathrm{~B}\left\{{ }^{1} \mathrm{H}\right\}$ NMR spectrum of compound $\mathbf{5 s}\left(\right.$ in $\mathrm{C}_{6} \mathrm{D}_{6}$ ). 


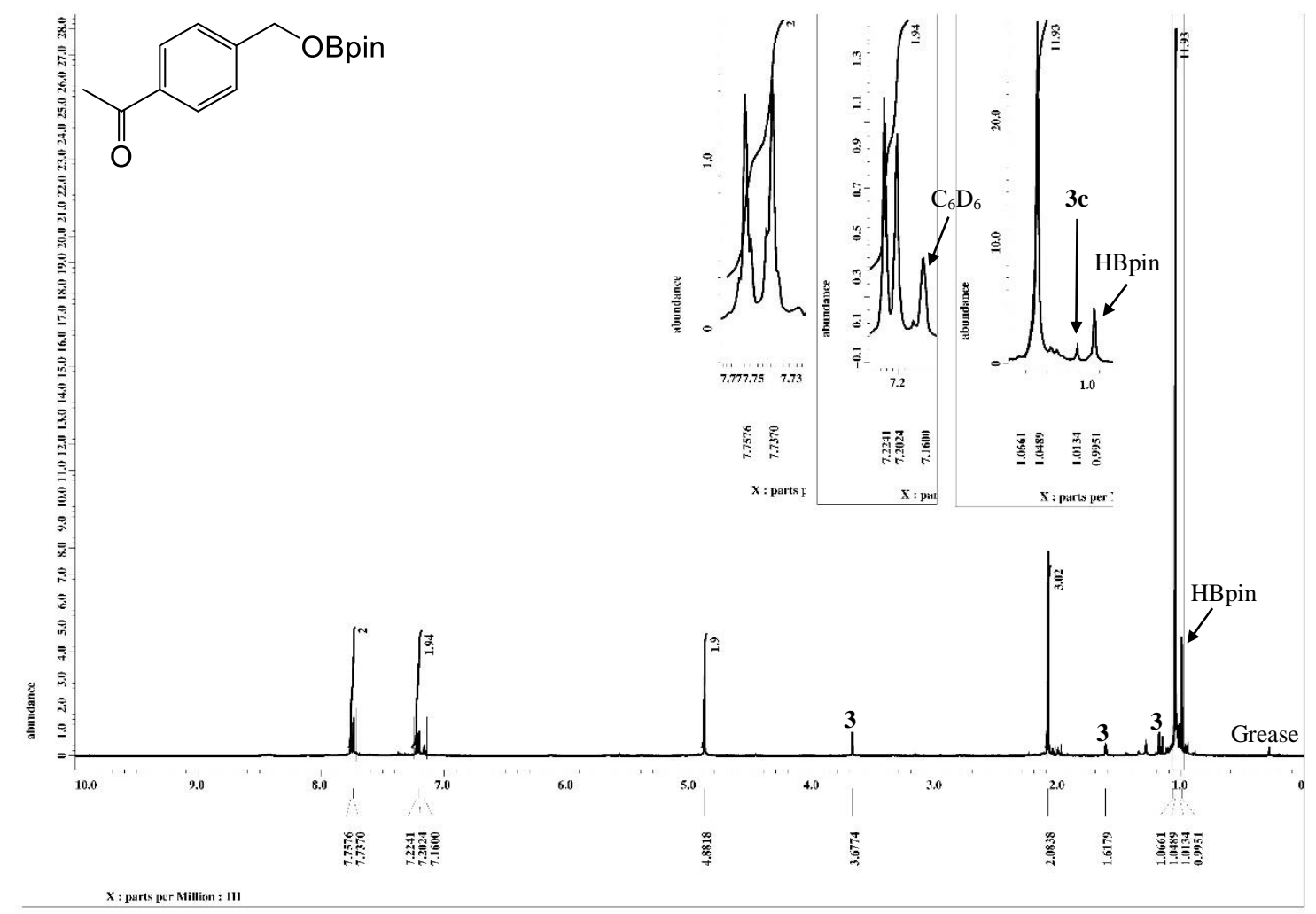

Figure S58. ${ }^{1} \mathrm{H}$ NMR spectrum of compound $5 t$ (in $\mathrm{C}_{6} \mathrm{D}_{6}$ ).

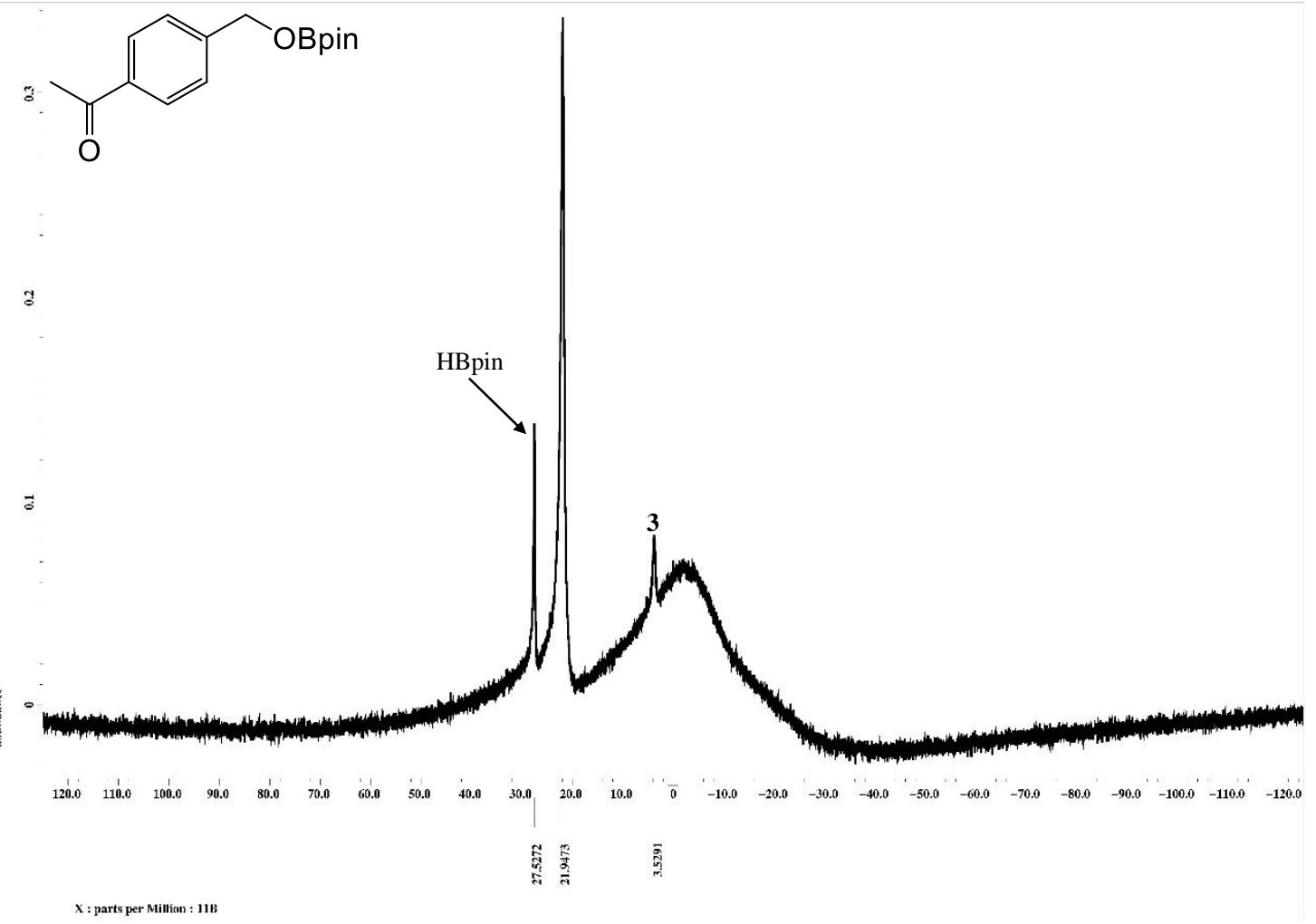

Figure S59. ${ }^{11} \mathrm{~B}\left\{{ }^{1} \mathrm{H}\right\}$ NMR spectrum of compound $\mathbf{5 t}$ (in $\mathrm{C}_{6} \mathrm{D}_{6}$ ). 


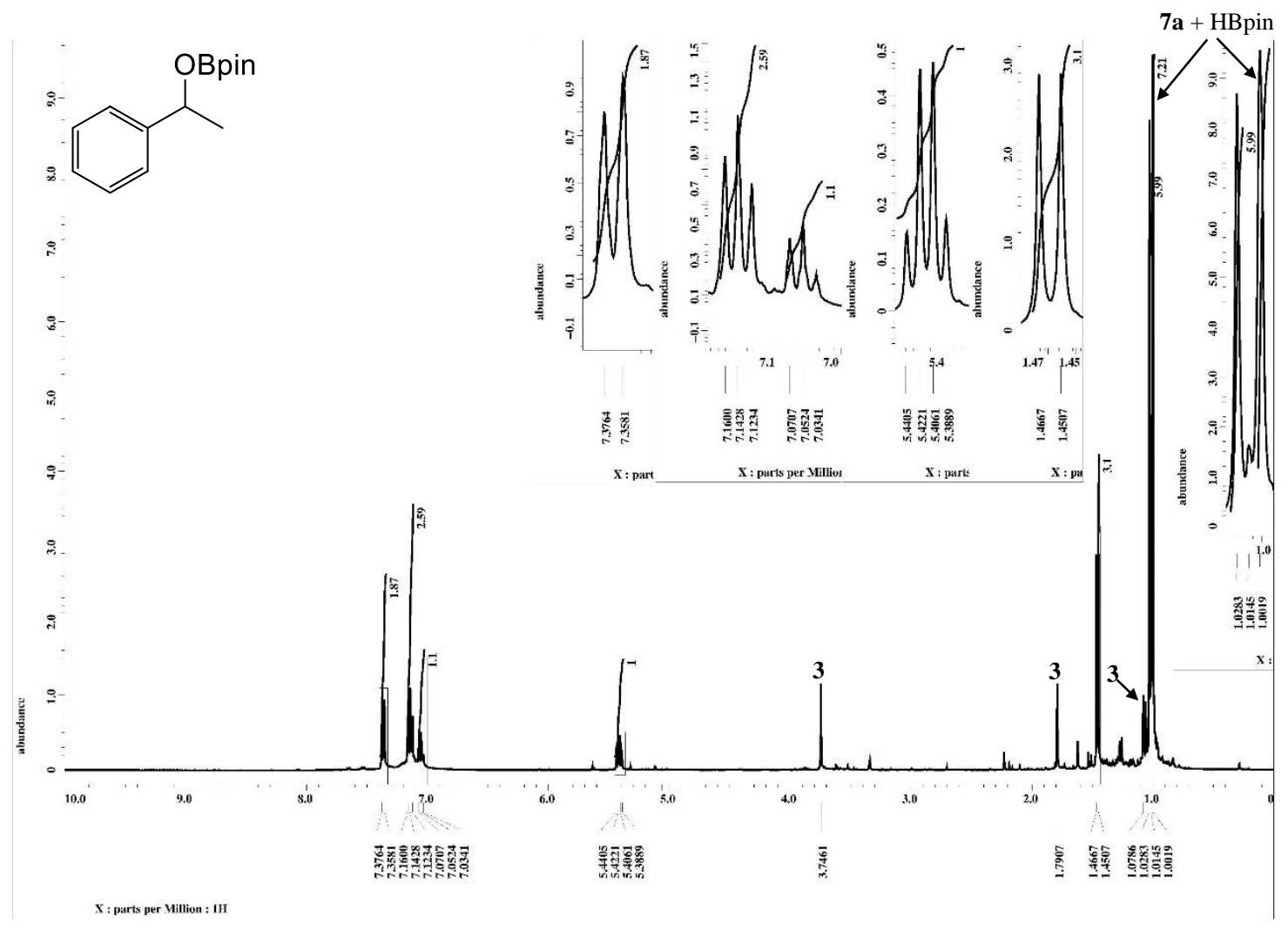

(a)

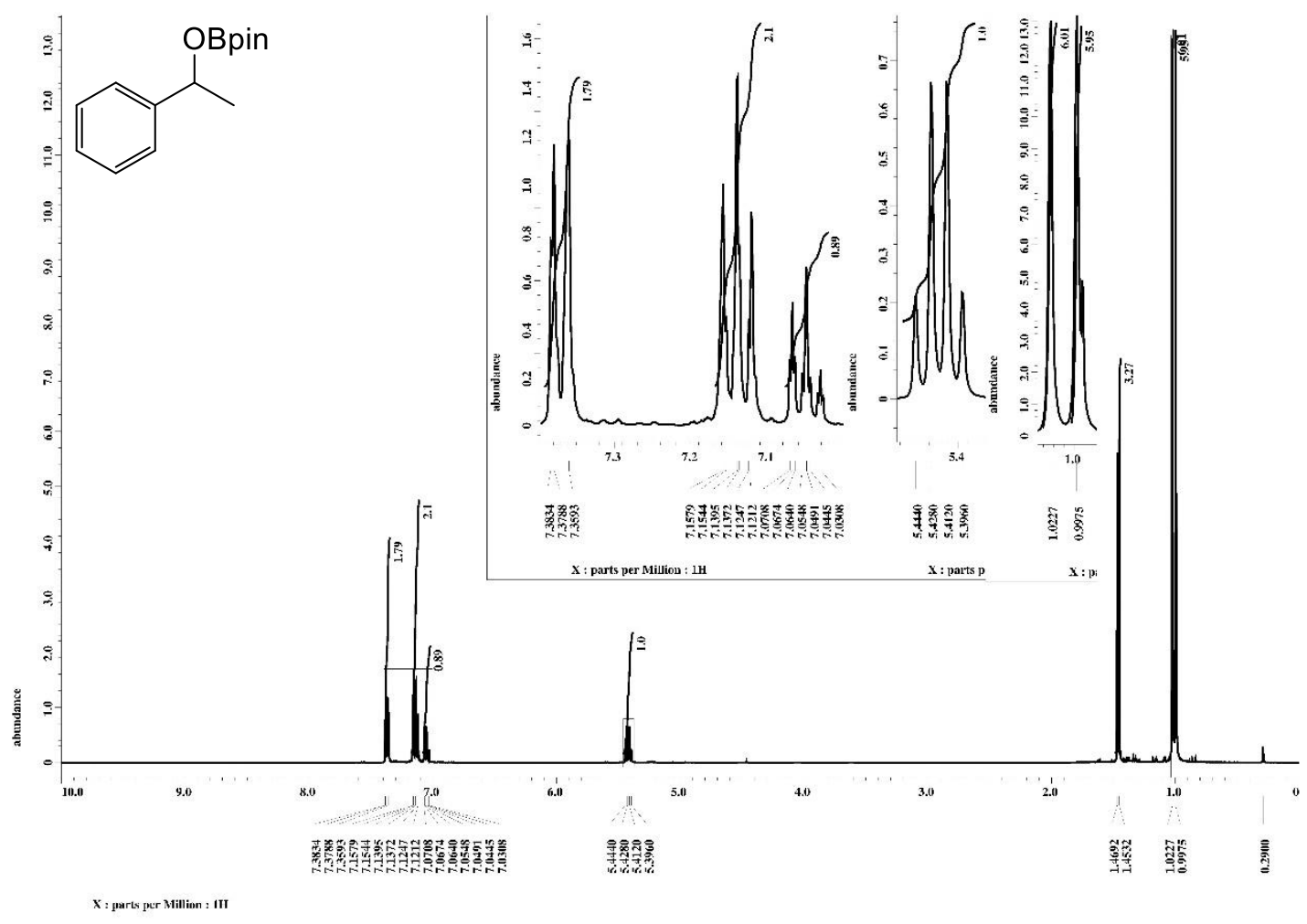

(b)

Figure S60. ${ }^{1} \mathrm{H}$ NMR spectrum of isolated compound 7a (in $\mathrm{C}_{6} \mathrm{D}_{6}$ ), (a) crude solution and (b) isolated compound. 


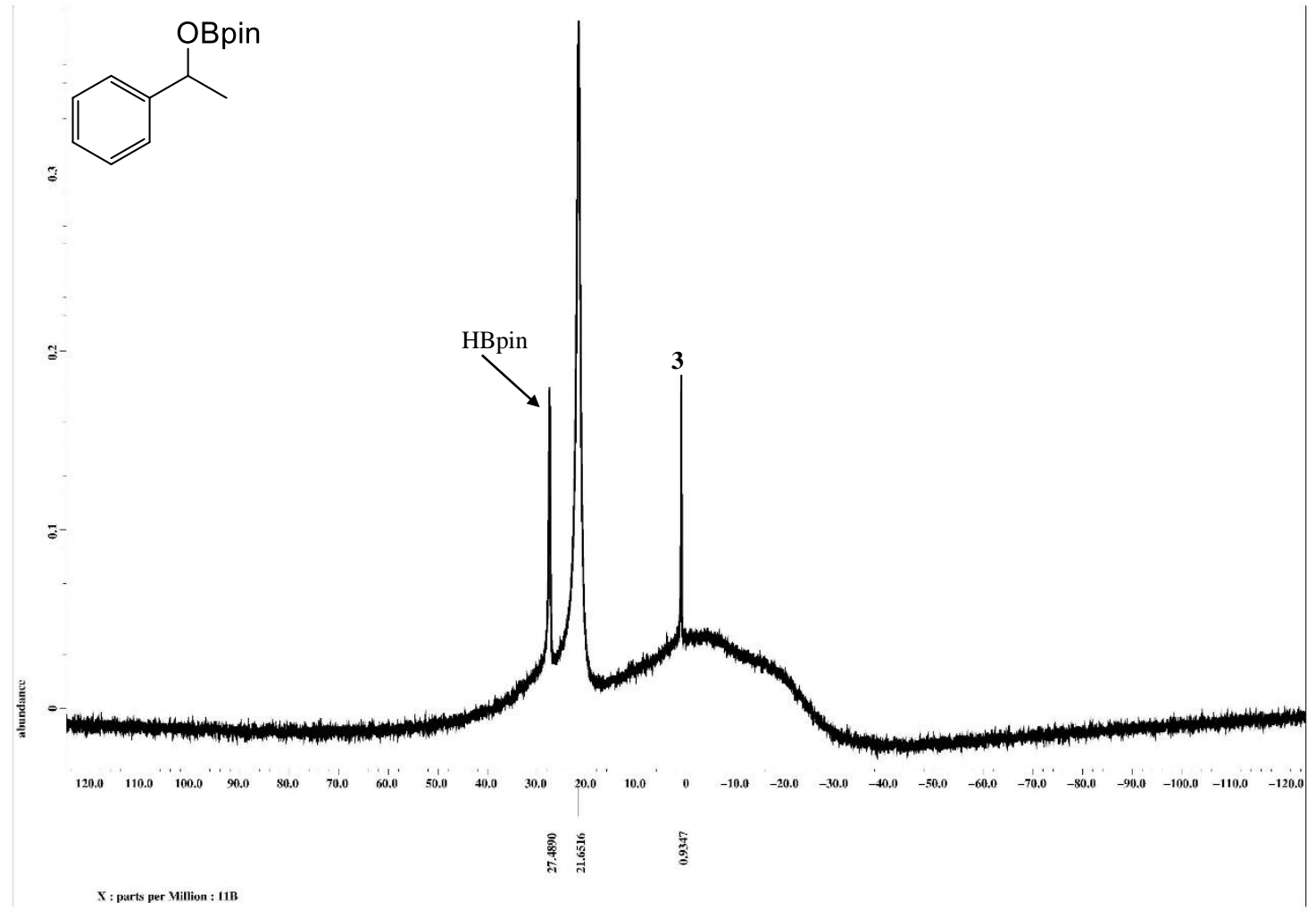

(a)

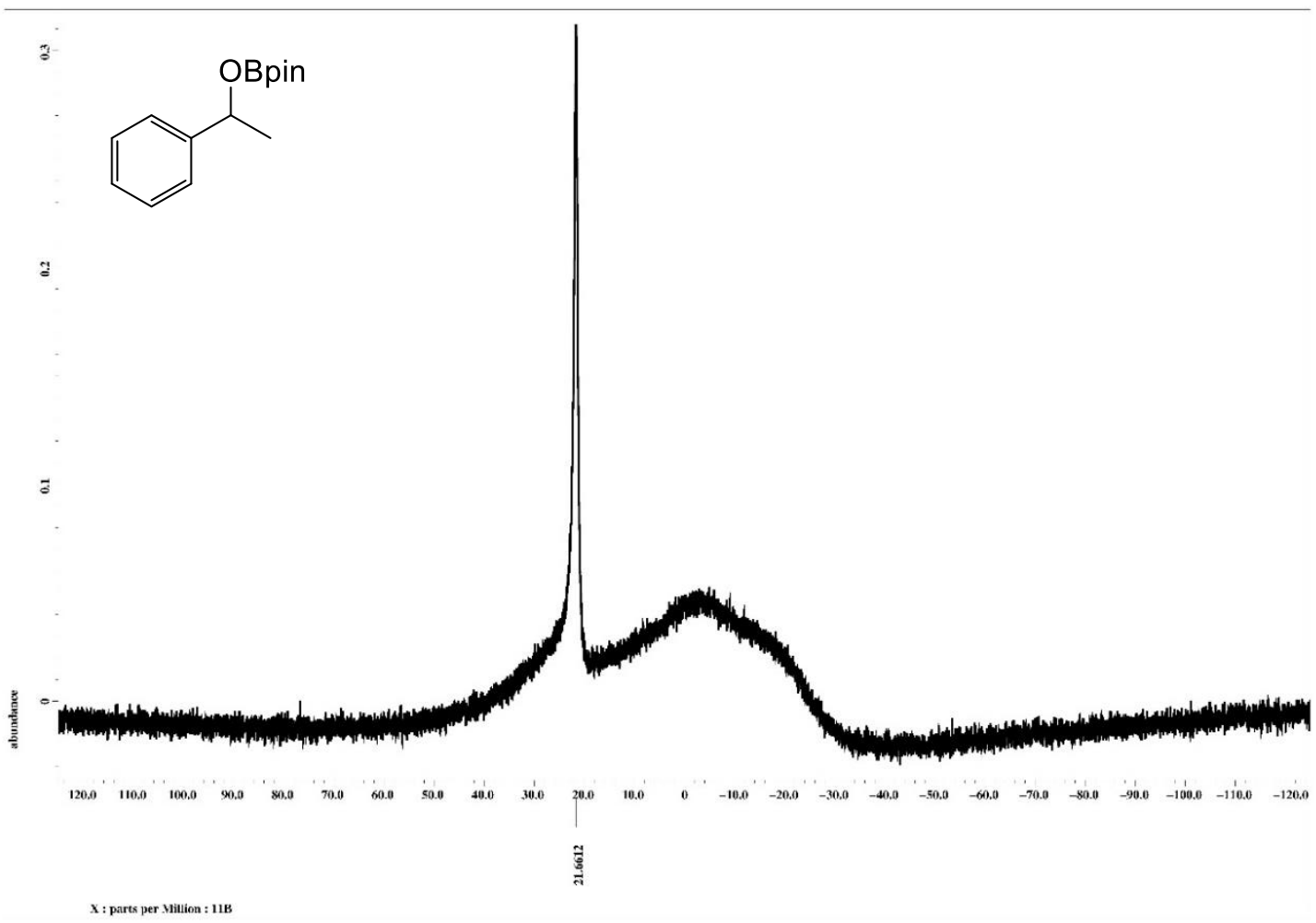

(b)

Figure S61. ${ }^{11} \mathrm{~B}\left\{{ }^{1} \mathrm{H}\right\}$ NMR spectrum of isolated compound 7a (in $\mathrm{C}_{6} \mathrm{D}_{6}$ ), (a) crude solution and (b) isolated compound. 


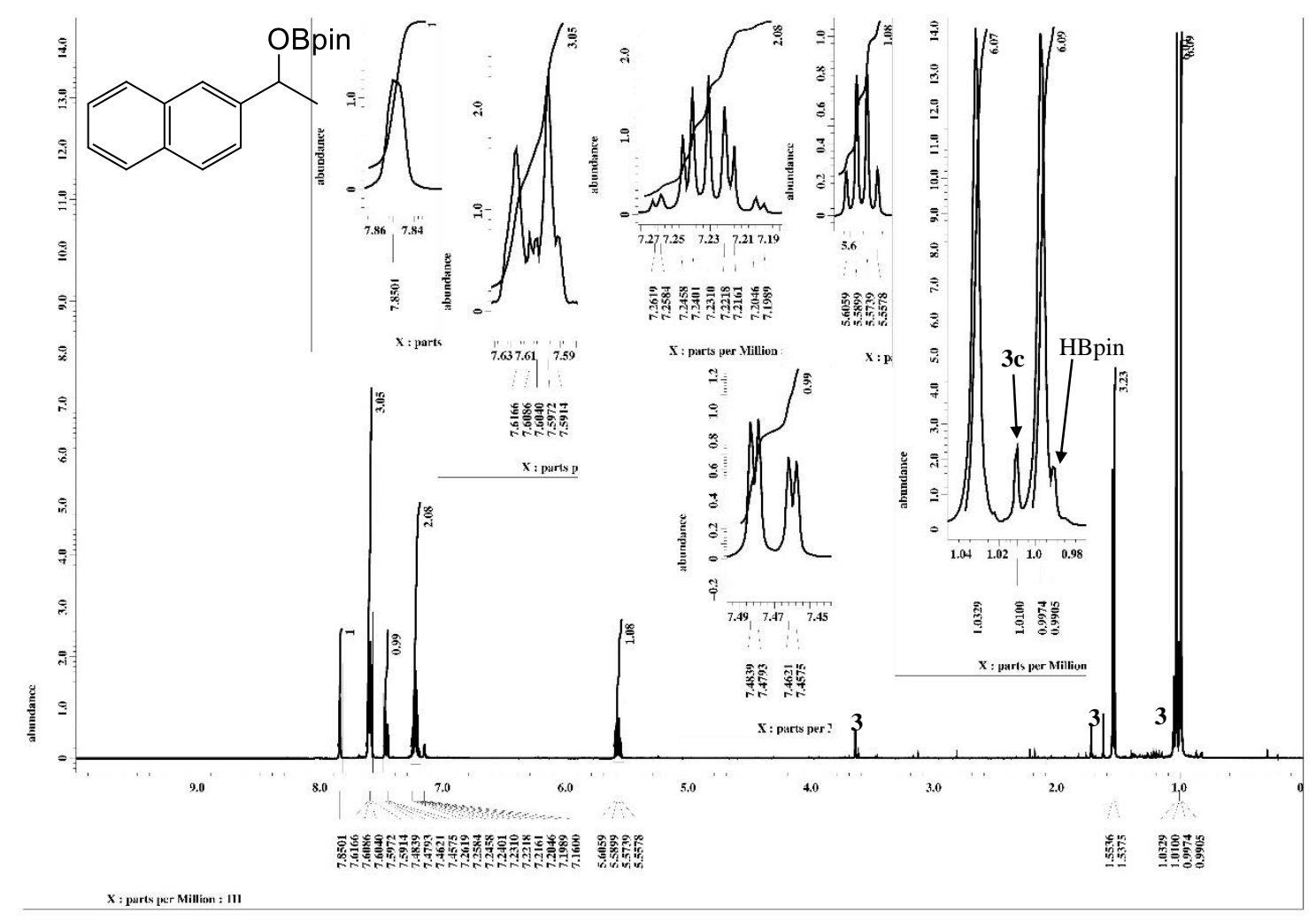

Figure S62. ${ }^{1} \mathrm{H}$ NMR spectrum of compound $7 \mathbf{b}$ (in $\mathrm{C}_{6} \mathrm{D}_{6}$ ).

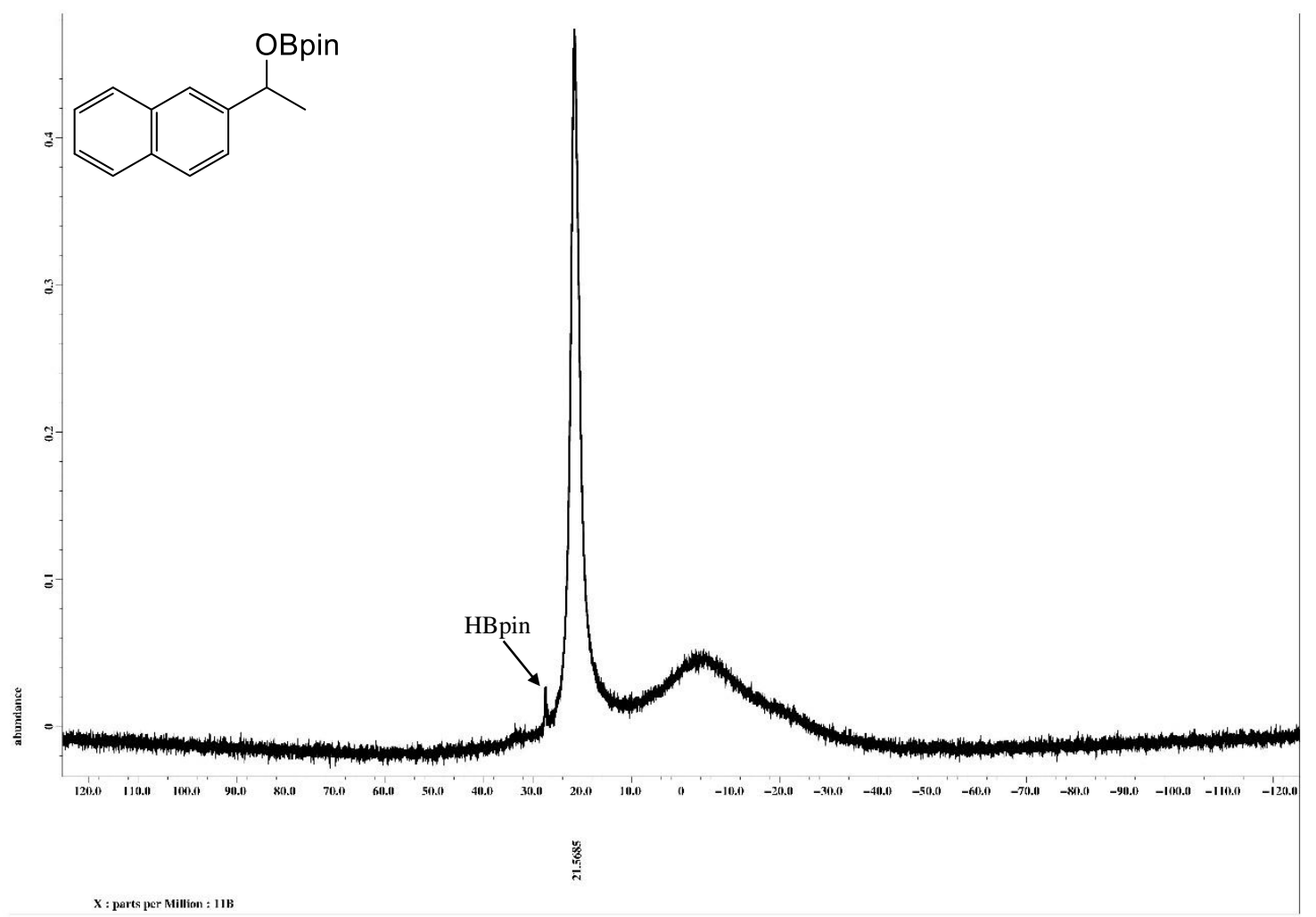

Figure S63. ${ }^{11} \mathrm{~B}\left\{{ }^{1} \mathrm{H}\right\}$ NMR spectrum of compound $7 \mathbf{b}$ (in $\mathrm{C}_{6} \mathrm{D}_{6}$ ). 


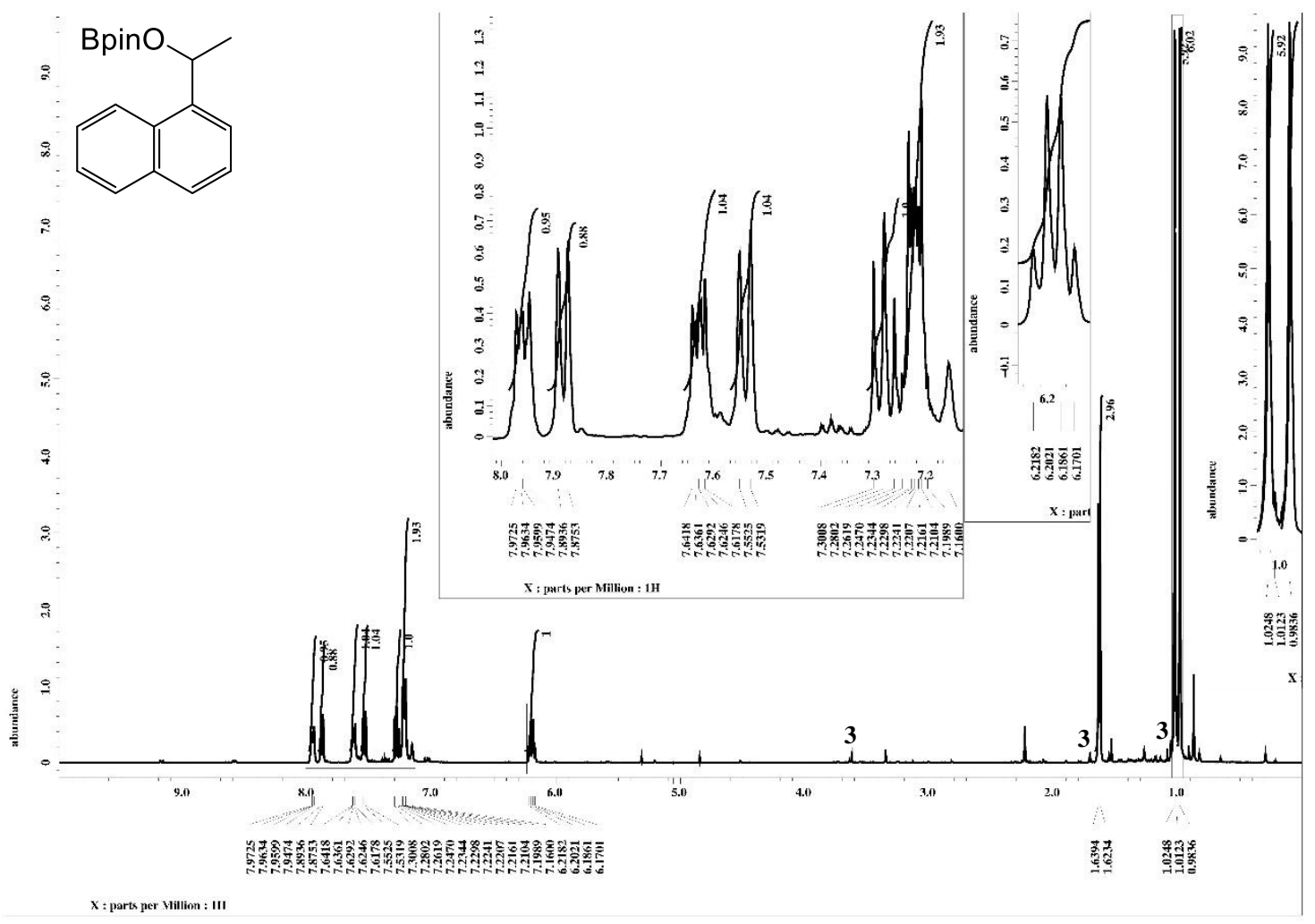

Figure S64. ${ }^{1} \mathrm{H}$ NMR spectrum of compound $7 \mathbf{c}$ (in $\mathrm{C}_{6} \mathrm{D}_{6}$ ).

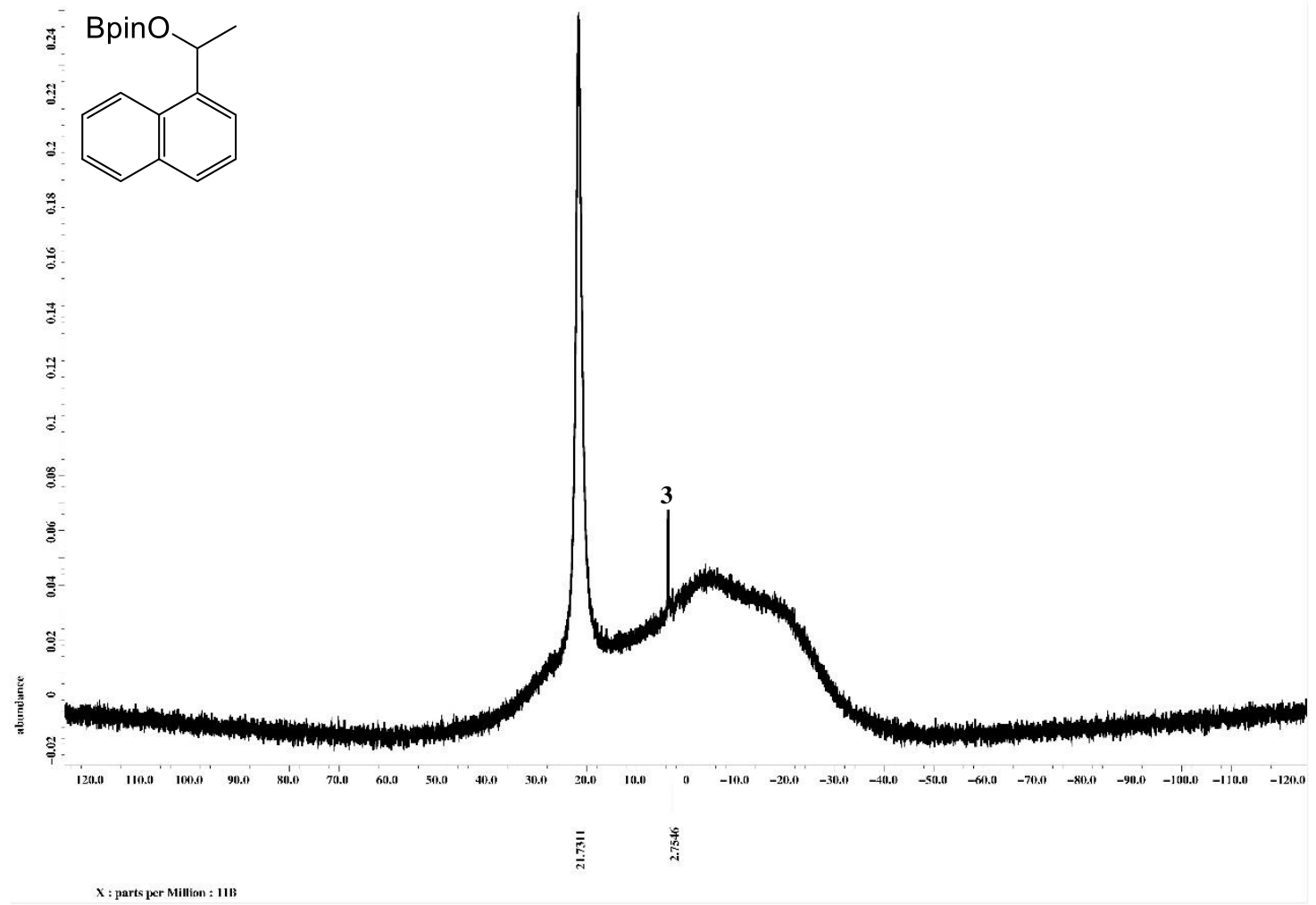

Figure S65. ${ }^{11} \mathrm{~B}\left\{{ }^{1} \mathrm{H}\right\}$ NMR spectrum of compound 7c (in $\left.\mathrm{C}_{6} \mathrm{D}_{6}\right)$. 


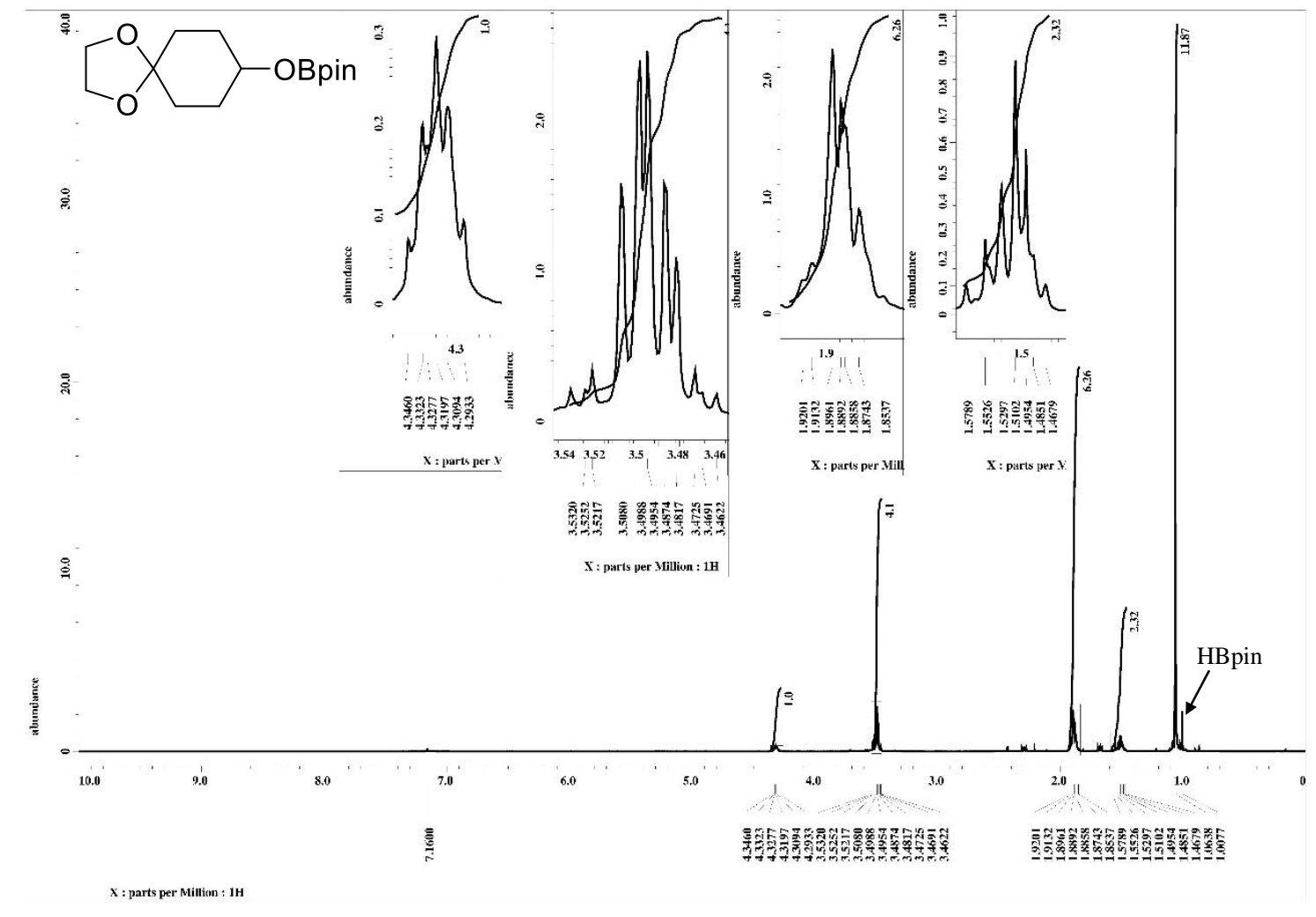

Figure S66. ${ }^{1} \mathrm{H}$ NMR spectrum of compound 7d (in $\mathrm{C}_{6} \mathrm{D}_{6}$ ).

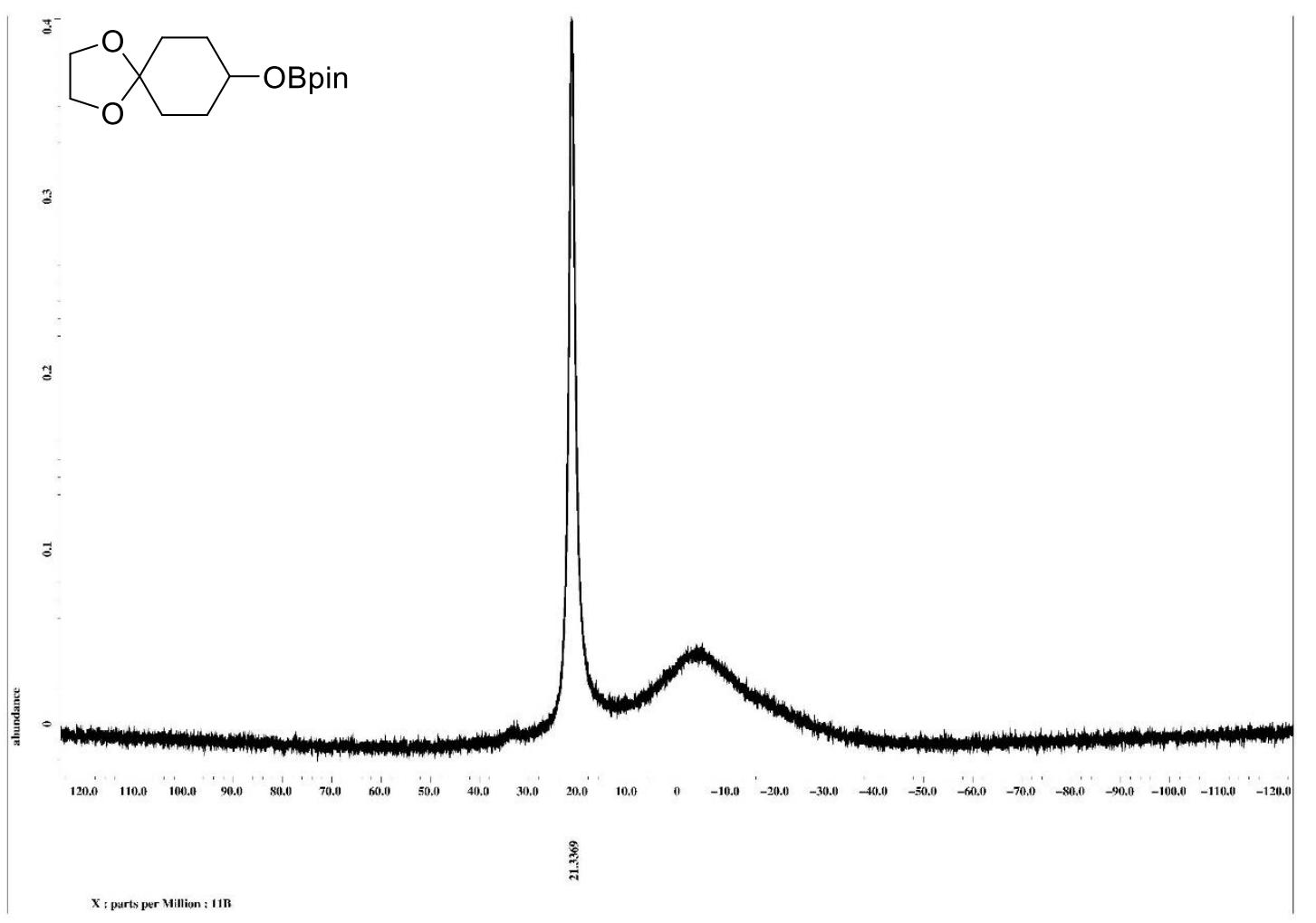

Figure S67. ${ }^{11} \mathrm{~B}\left\{{ }^{1} \mathrm{H}\right\}$ NMR spectrum of compound 7d (in $\mathrm{C}_{6} \mathrm{D}_{6}$ ). 


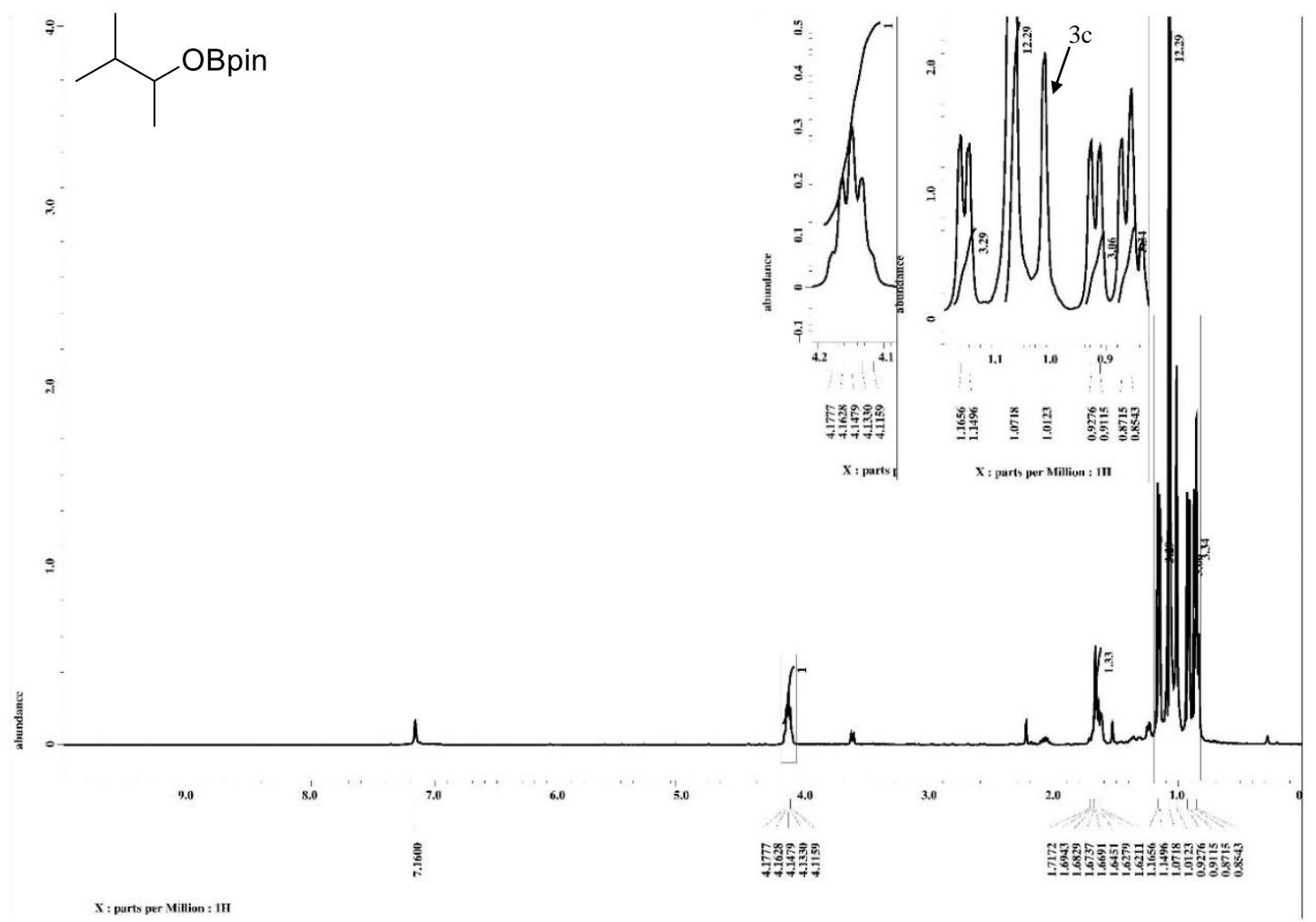

(a)

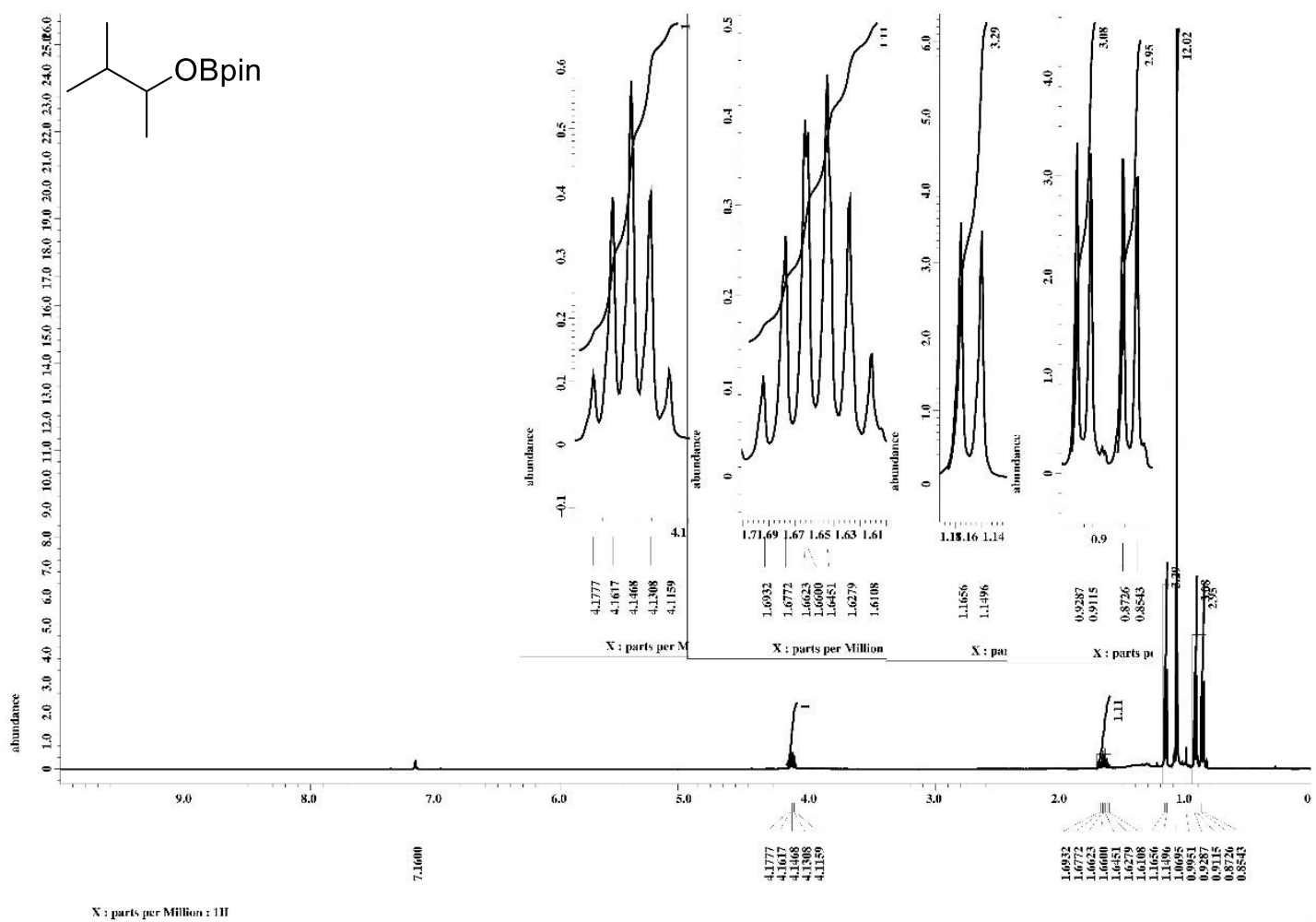

(b)

Figure S68. ${ }^{1} \mathrm{H}$ NMR spectrum of compound 7e (in $\mathrm{C}_{6} \mathrm{D}_{6}$ ), (a) crude solution and (b) isolated compound. 


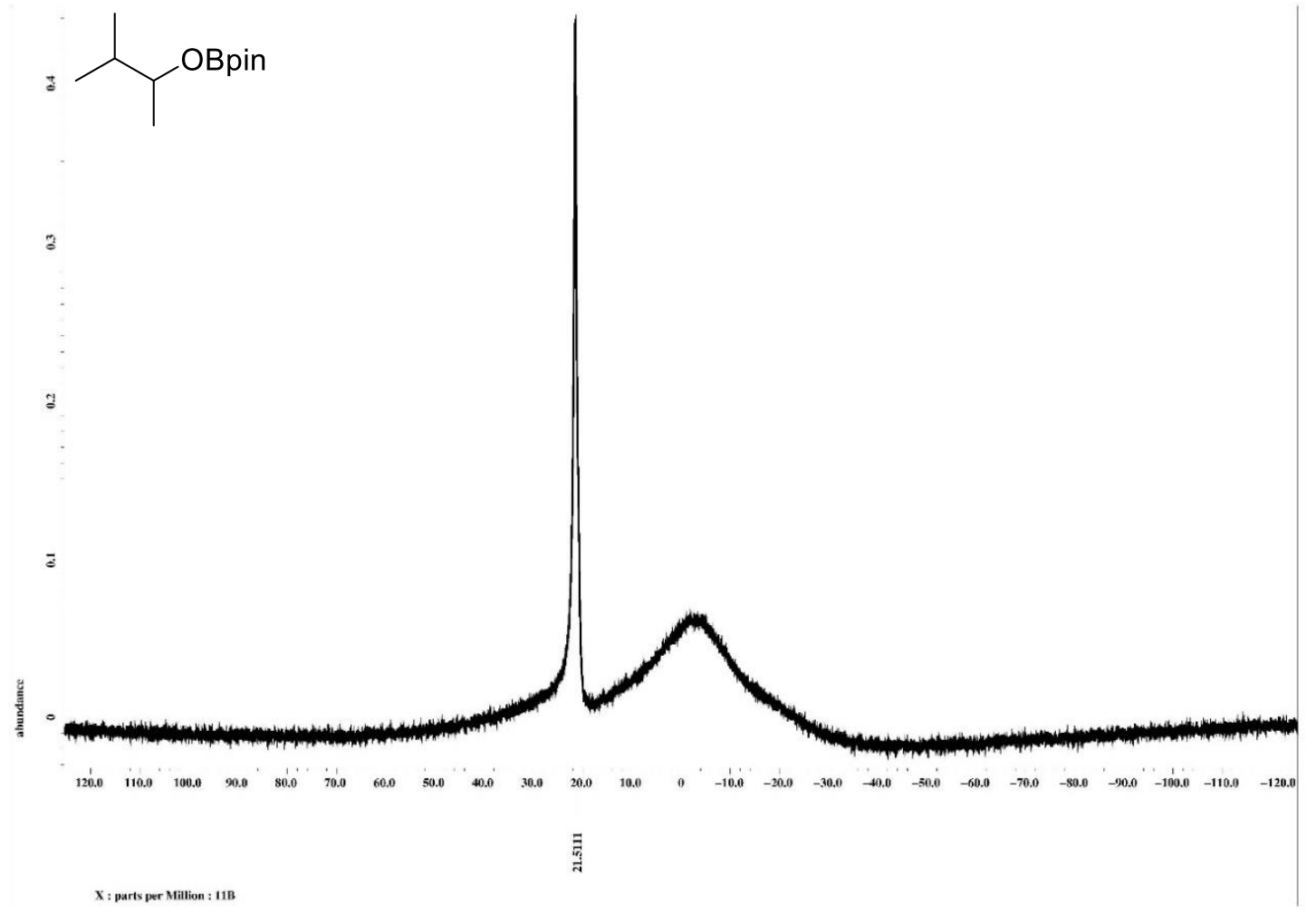

(a)

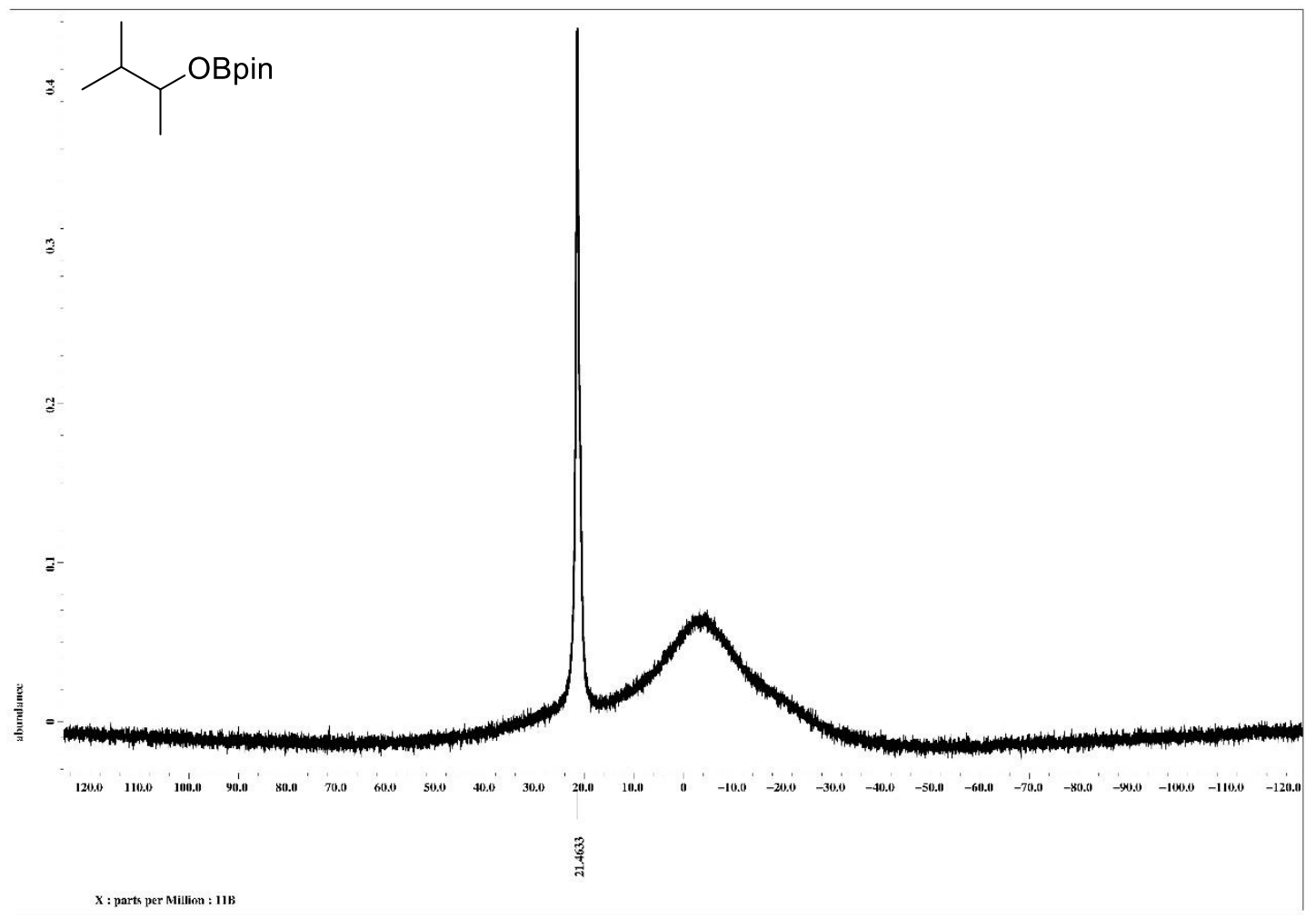

(b)

Figure S69. ${ }^{11} \mathrm{~B}\left\{{ }^{1} \mathrm{H}\right\}$ NMR spectrum of compound 7e (in $\mathrm{C}_{6} \mathrm{D}_{6}$ ), (a) crude solution and (b) isolated compound. 


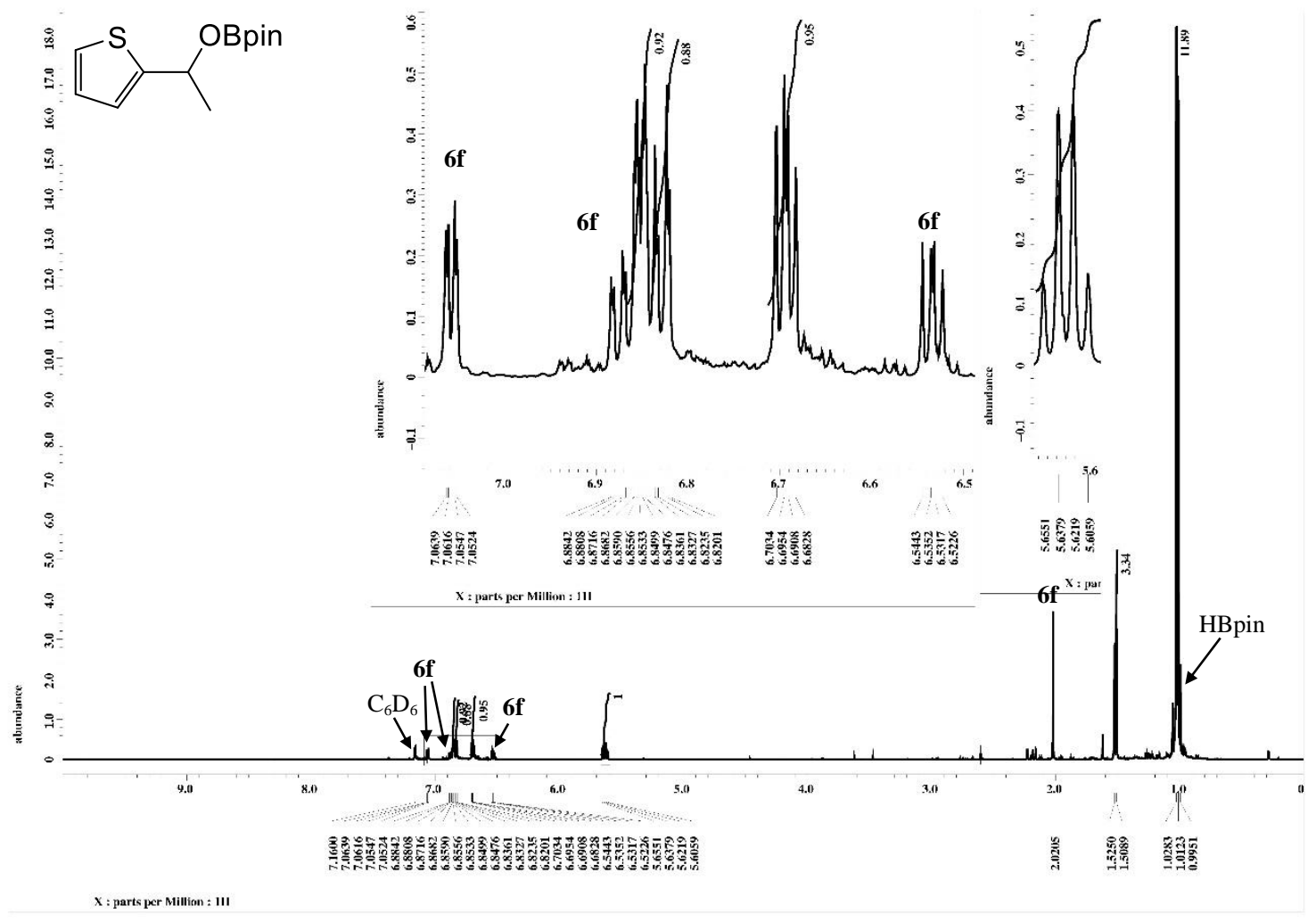

(a)

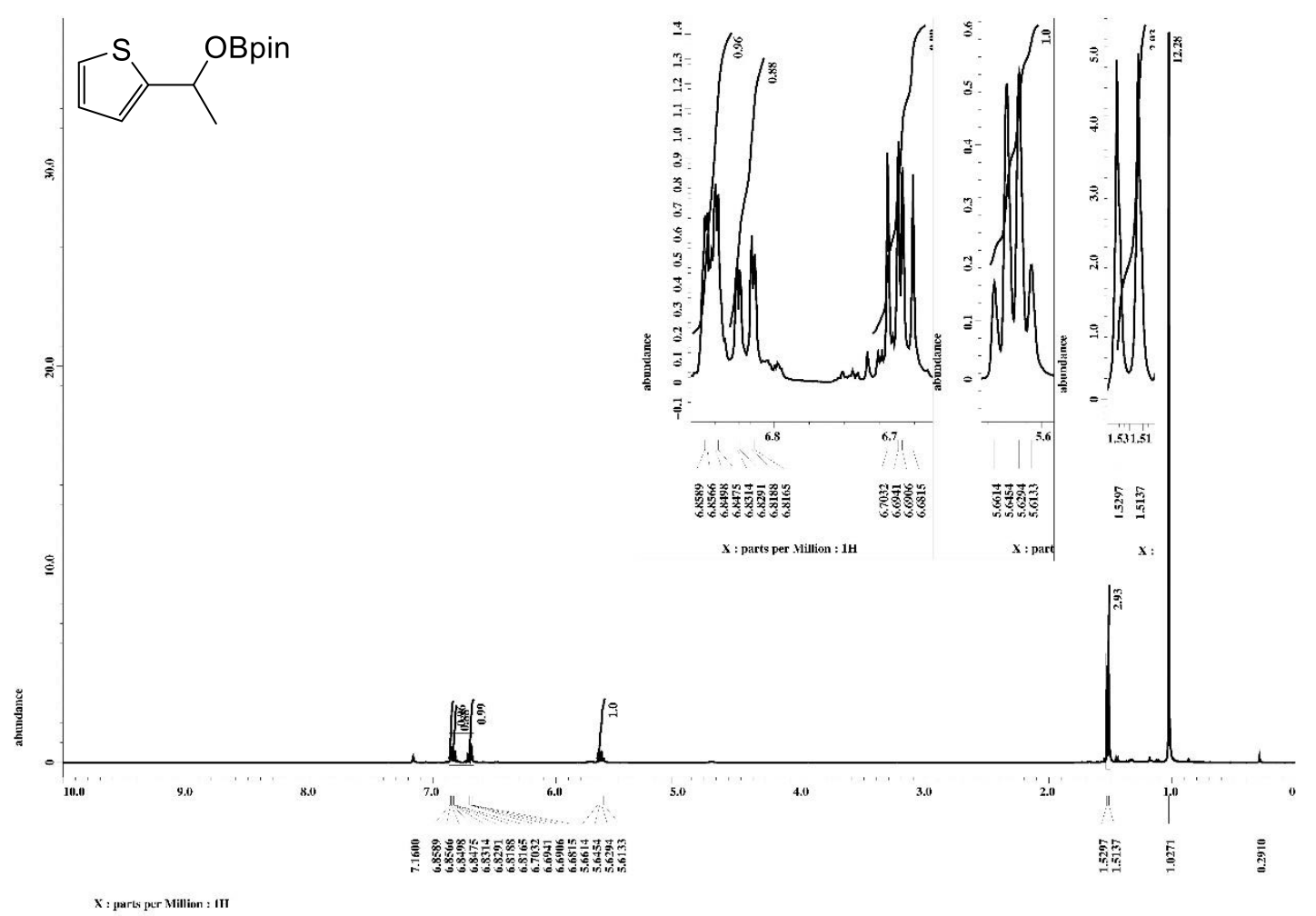

(b)

Figure S70. ${ }^{1} \mathrm{H}$ NMR spectrum of compound 7f (in $\mathrm{C}_{6} \mathrm{D}_{6}$ ), (a) crude solution and (b) isolated compound. 


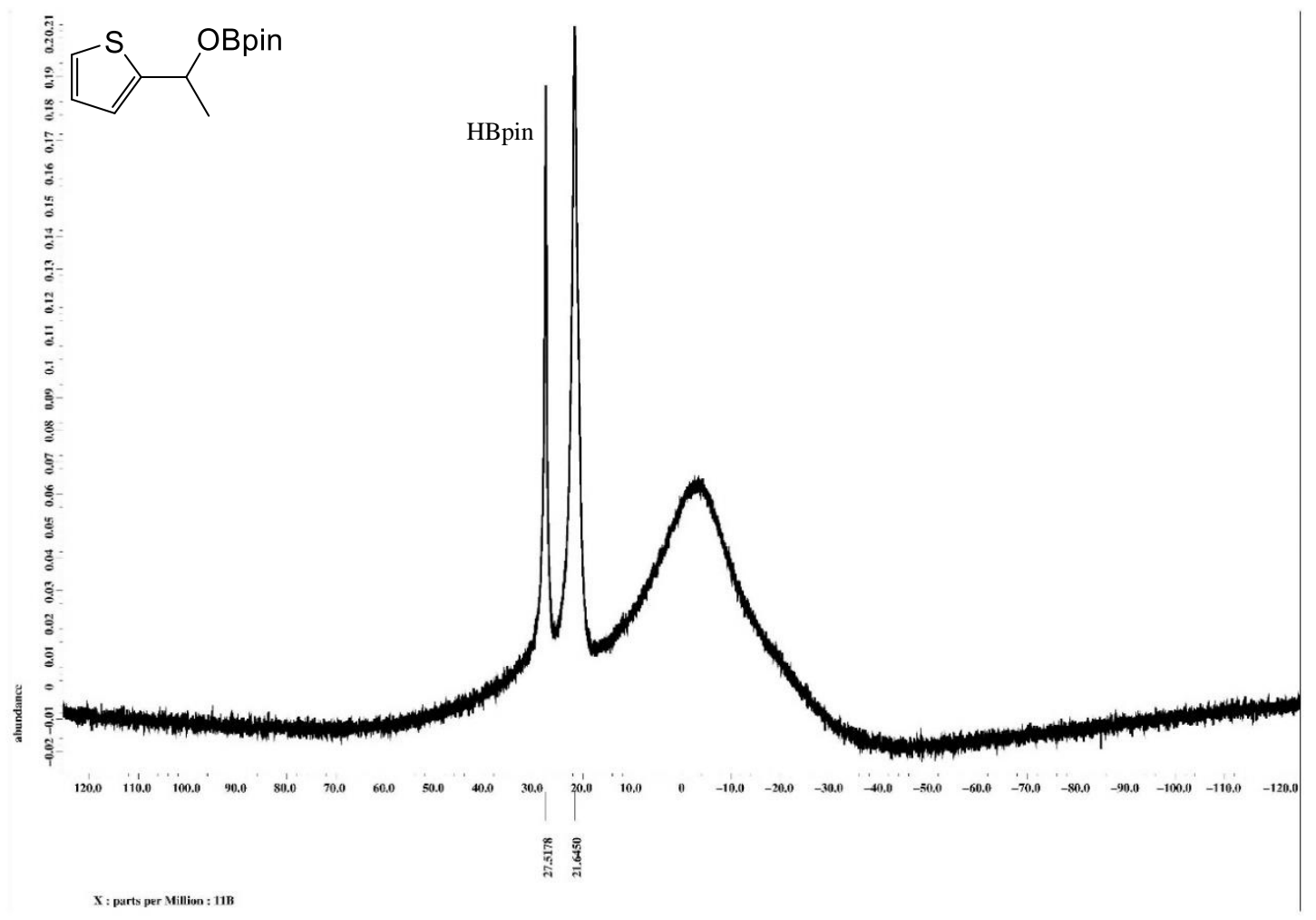

(a)

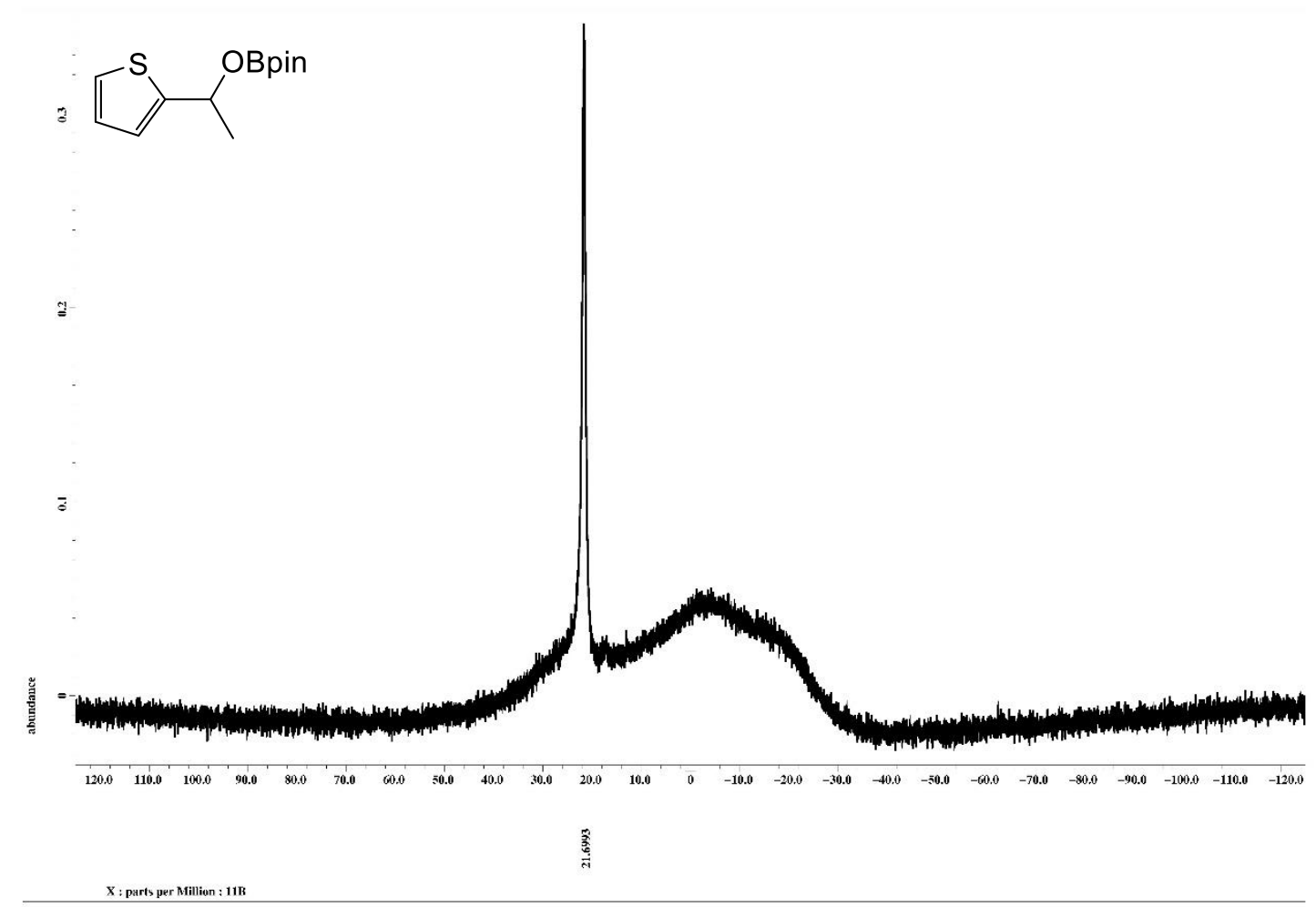

(b)

Figure S71. ${ }^{11} \mathrm{~B}\left\{{ }^{1} \mathrm{H}\right\}$ NMR spectrum of compound $7 \mathbf{f}$ (in $\mathrm{C}_{6} \mathrm{D}_{6}$ ), (a) crude solution and (b) isolated compound. 


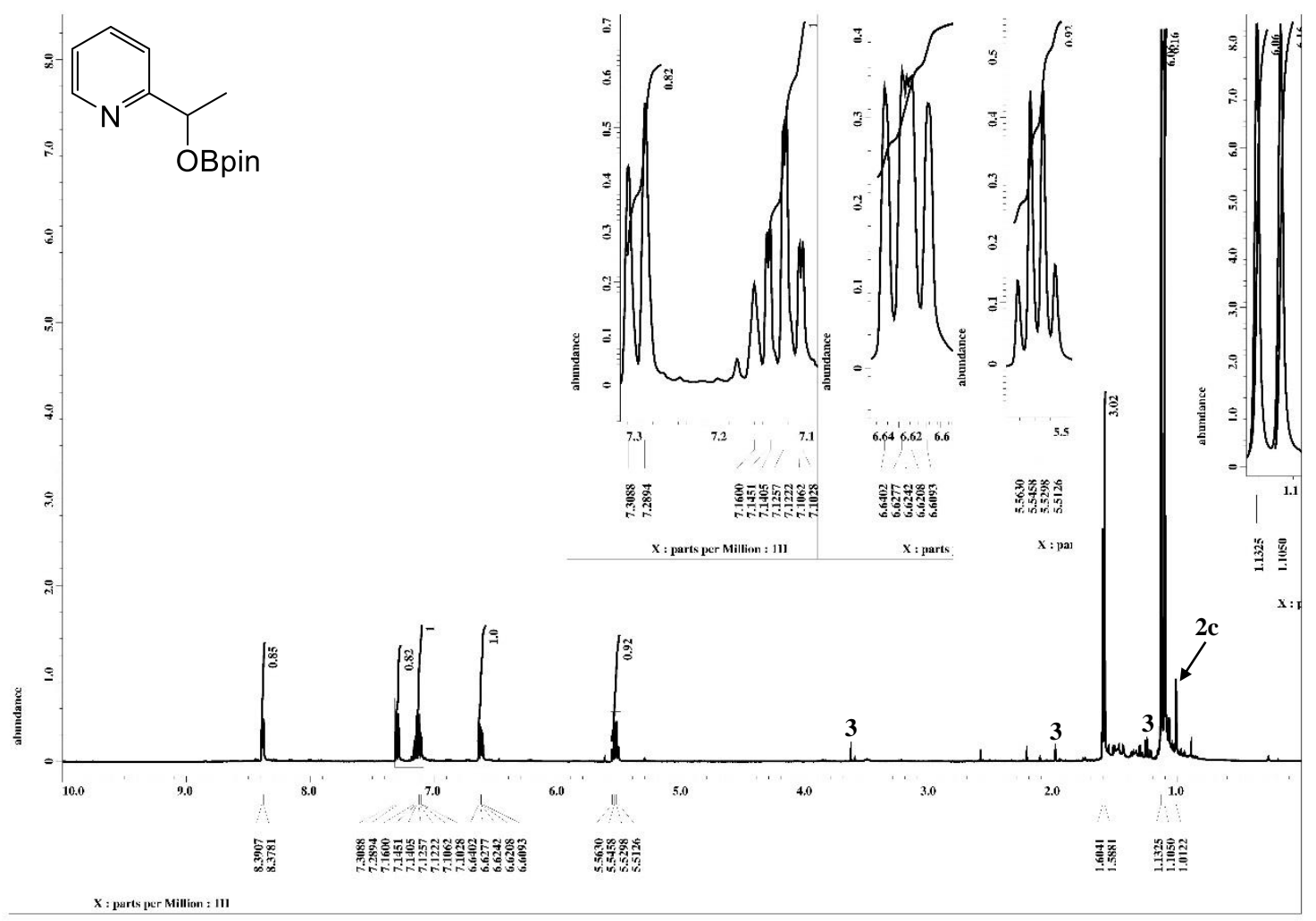

Figure S72. ${ }^{1} \mathrm{H}$ NMR spectrum of compound $7 \mathbf{g}$ (in $\mathrm{C}_{6} \mathrm{D}_{6}$ ).

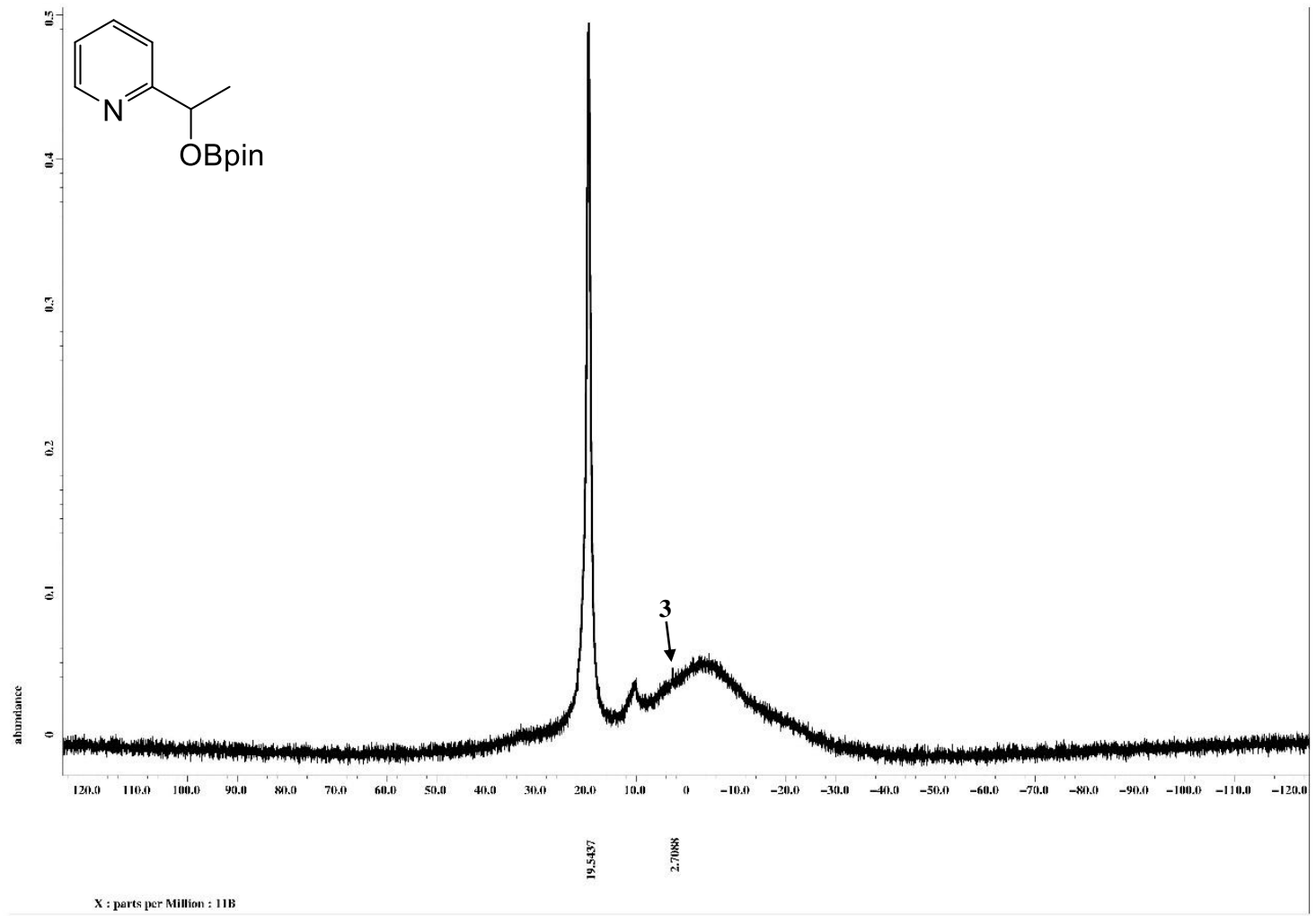

Figure S73. ${ }^{11} \mathrm{~B}\left\{{ }^{1} \mathrm{H}\right\}$ NMR spectrum of compound $7 \mathbf{g}$ (in $\mathrm{C}_{6} \mathrm{D}_{6}$ ). 


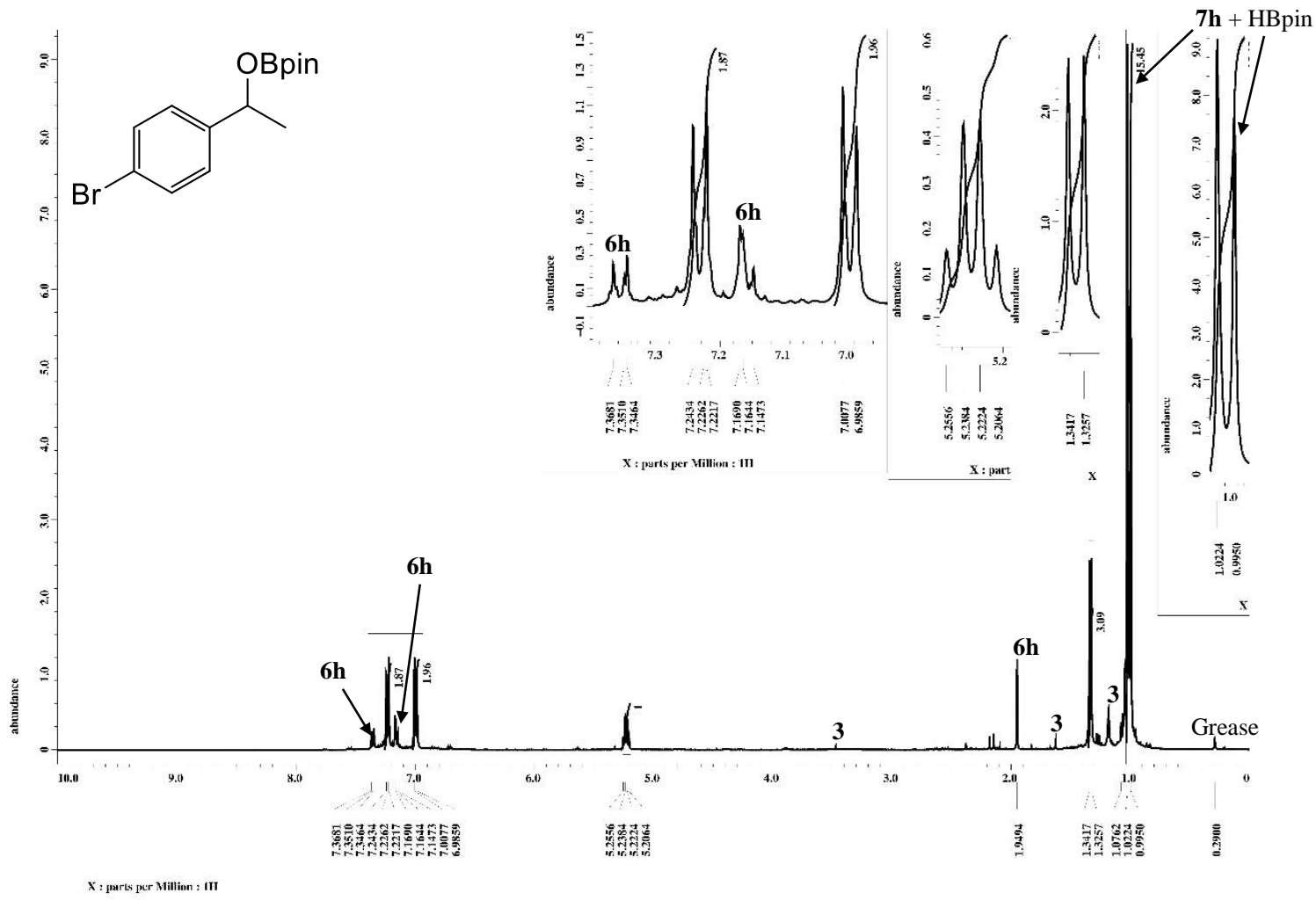

(a)

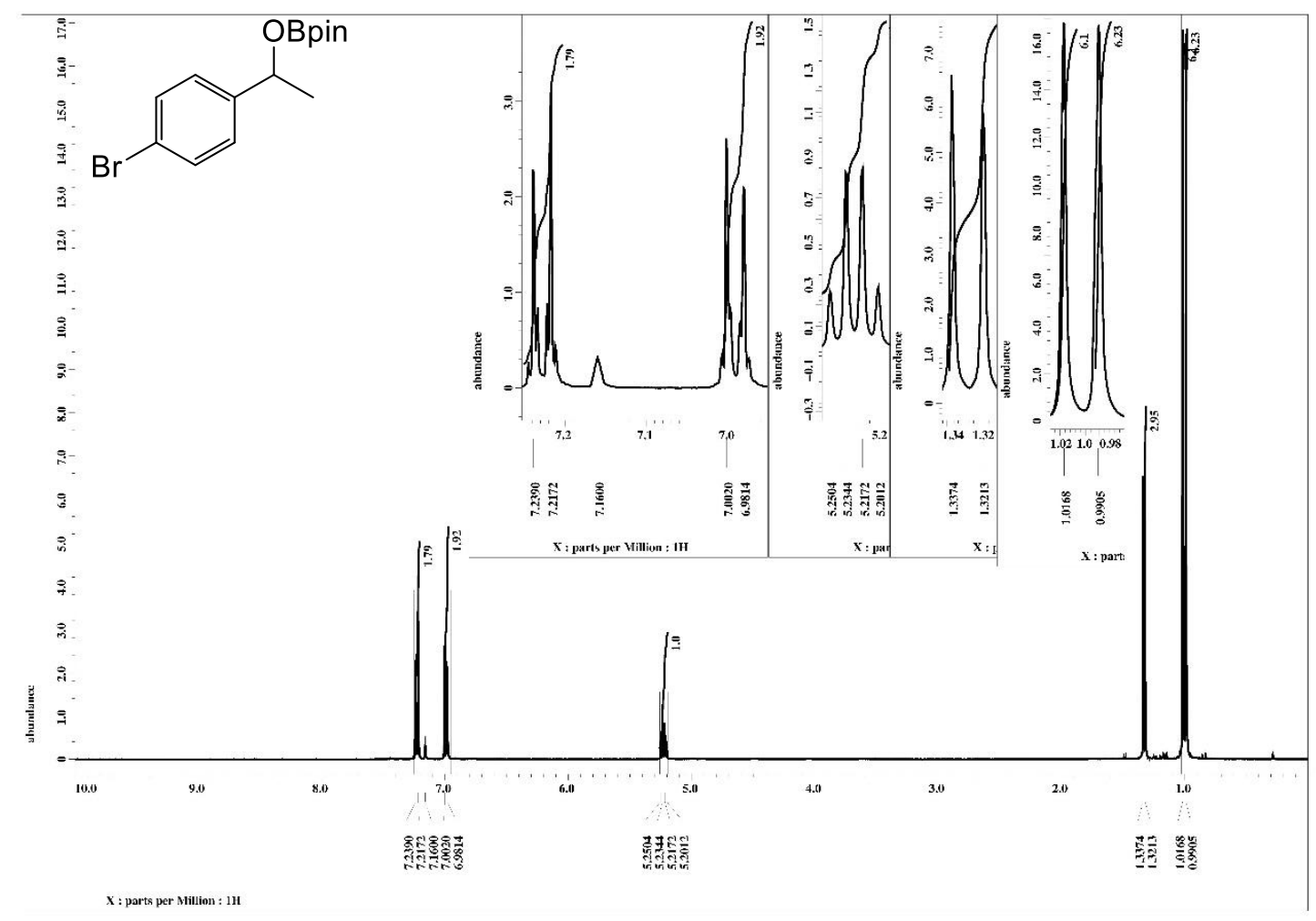

(b)

Figure S74. ${ }^{1} \mathrm{H}$ NMR spectrum of isolated compound 7 h (in $\mathrm{C}_{6} \mathrm{D}_{6}$ ), (a) crude solution and (b) isolated compound. 


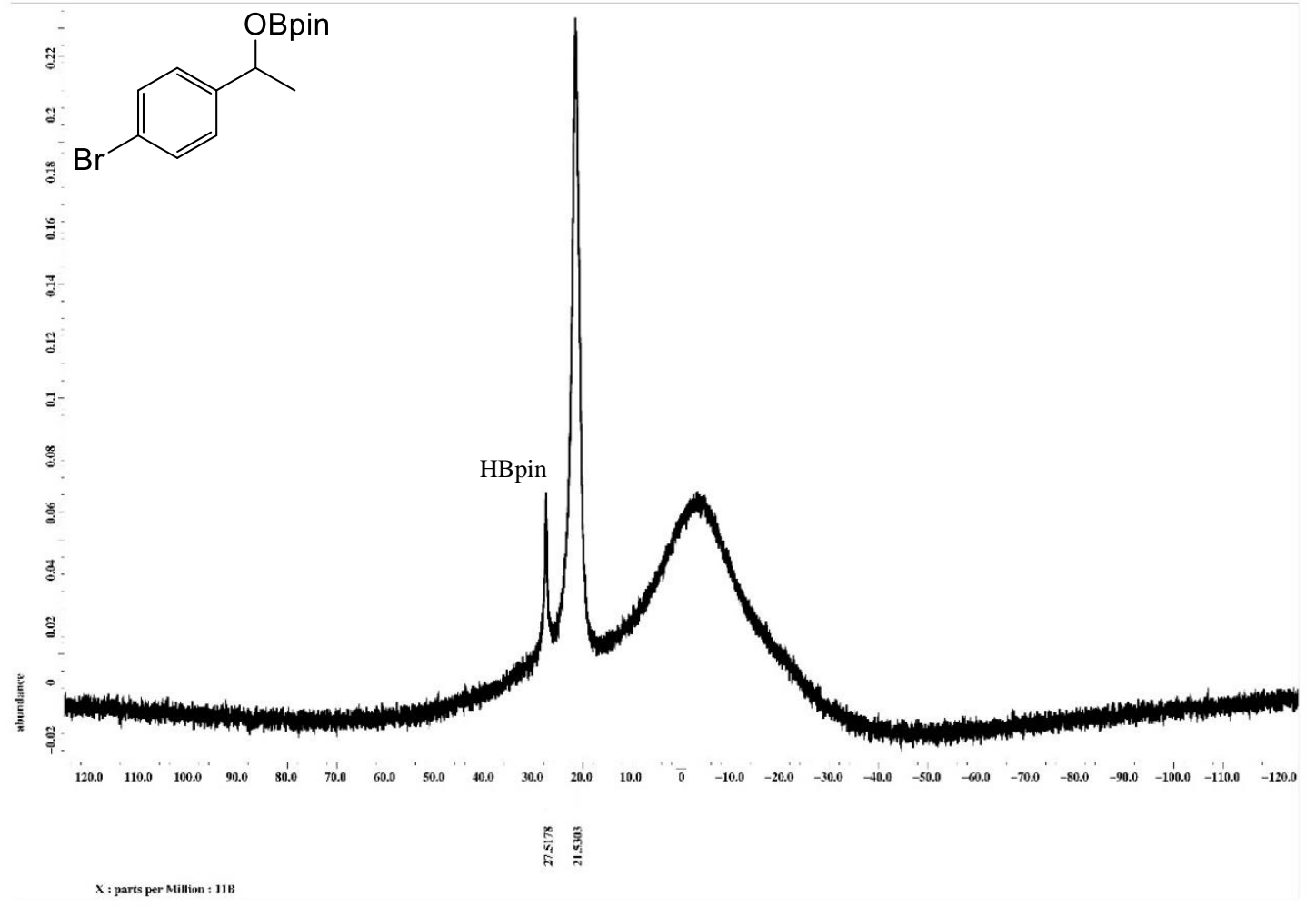

(a)

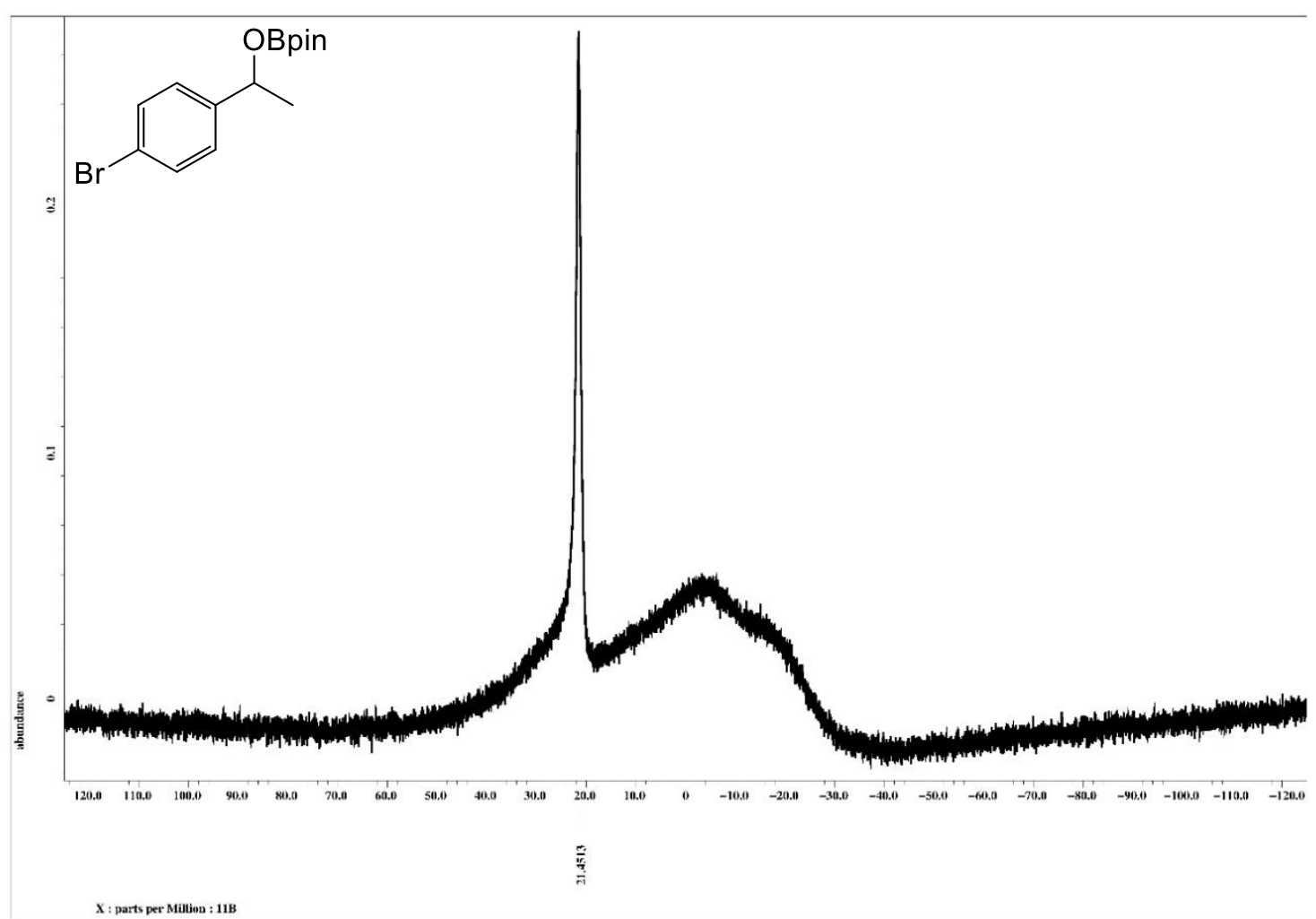

(b)

Figure S75. ${ }^{11} \mathrm{~B}\left\{{ }^{1} \mathrm{H}\right\}$ NMR spectrum of isolated compound $7 \mathbf{h}$ (in $\mathrm{C}_{6} \mathrm{D}_{6}$ ), (a) crude solution and (b) isolated compound.

Page S82 


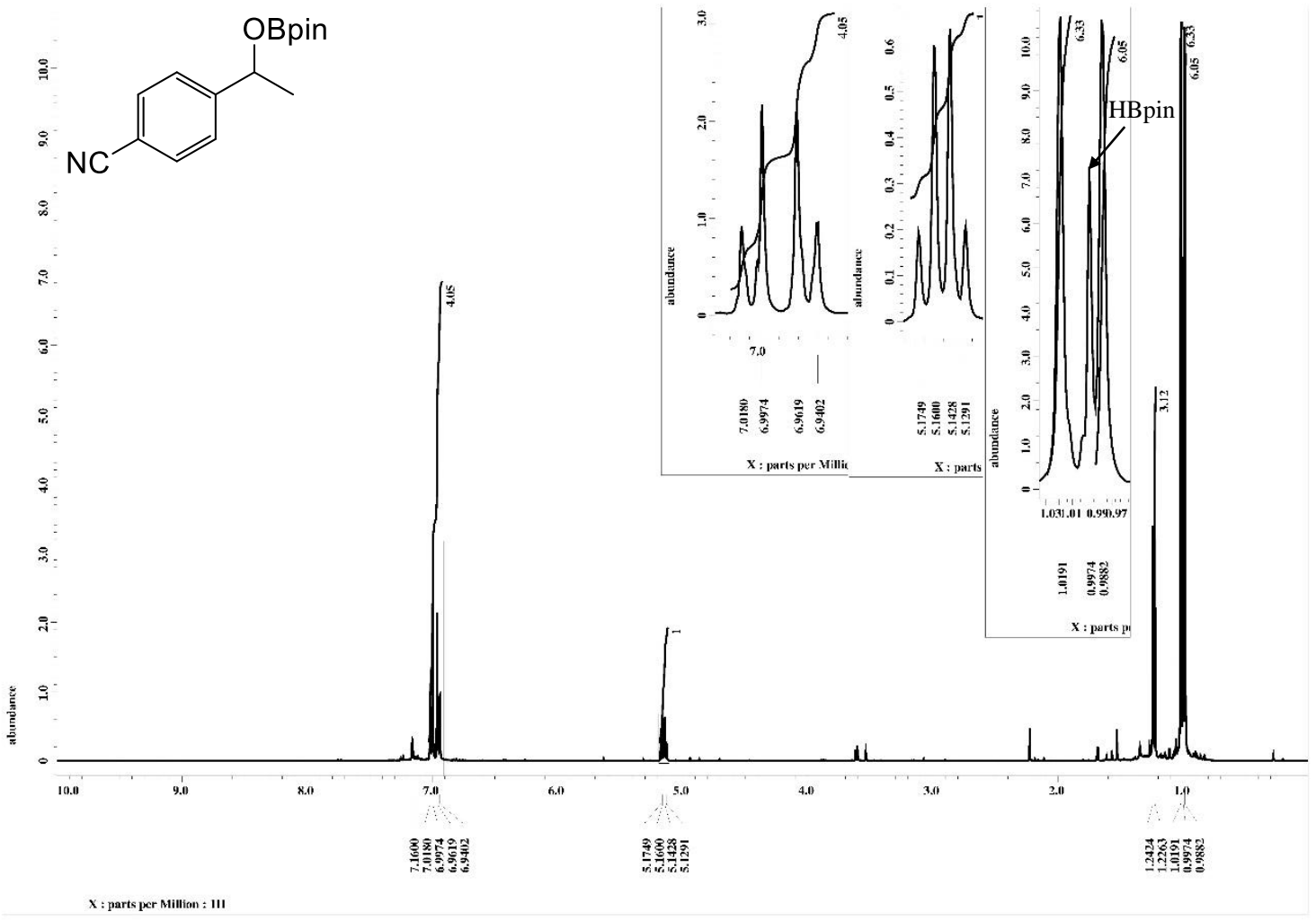

Figure S76. ${ }^{1} \mathrm{H}$ NMR spectrum of compound $7 \mathbf{j}$ (in $\mathrm{C}_{6} \mathrm{D}_{6}$ ).

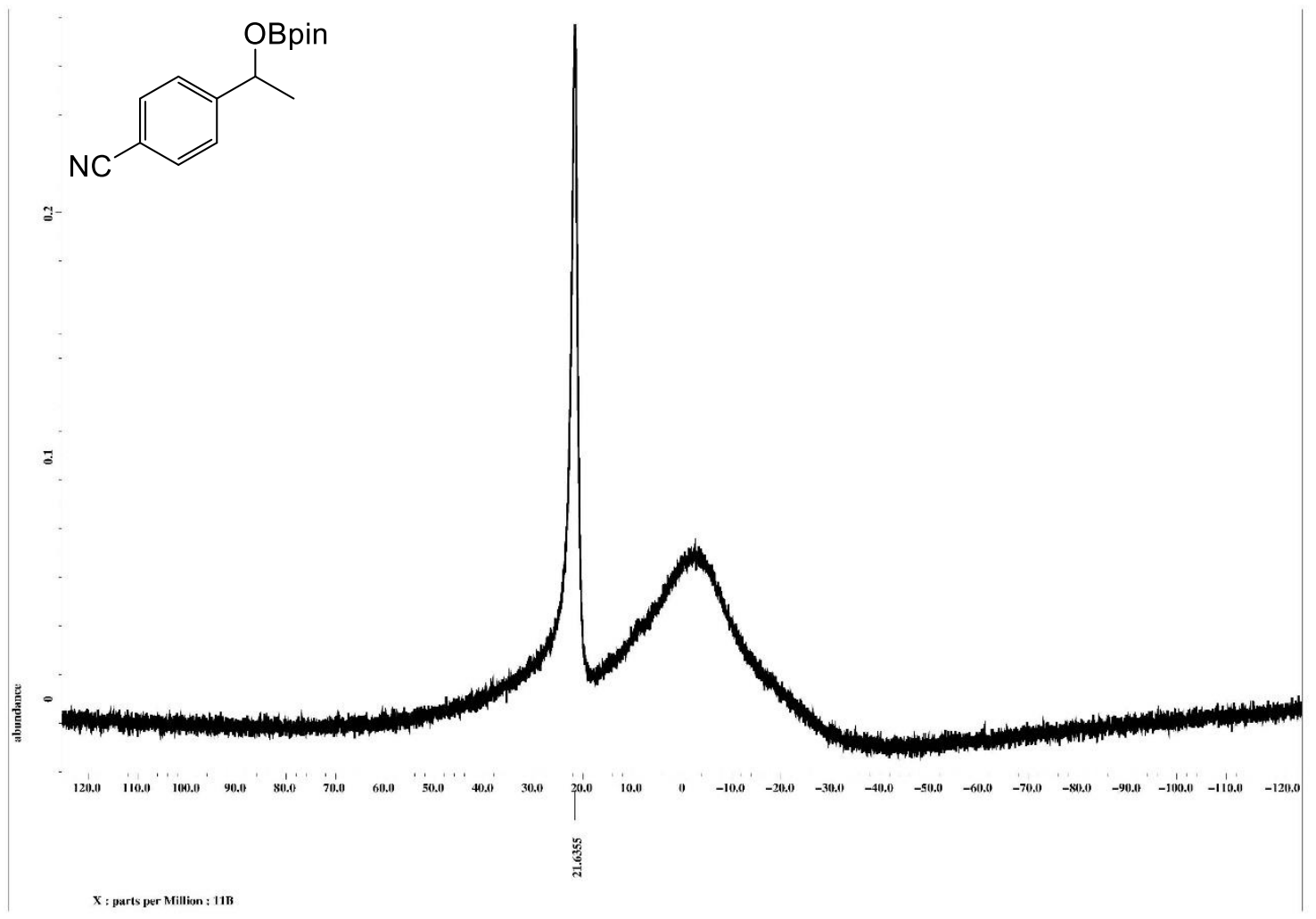

Figure S77. ${ }^{11} \mathrm{~B}\left\{{ }^{1} \mathrm{H}\right\}$ NMR spectrum of compound $\mathbf{7 j}$ (in $\mathrm{C}_{6} \mathrm{D}_{6}$ ). 


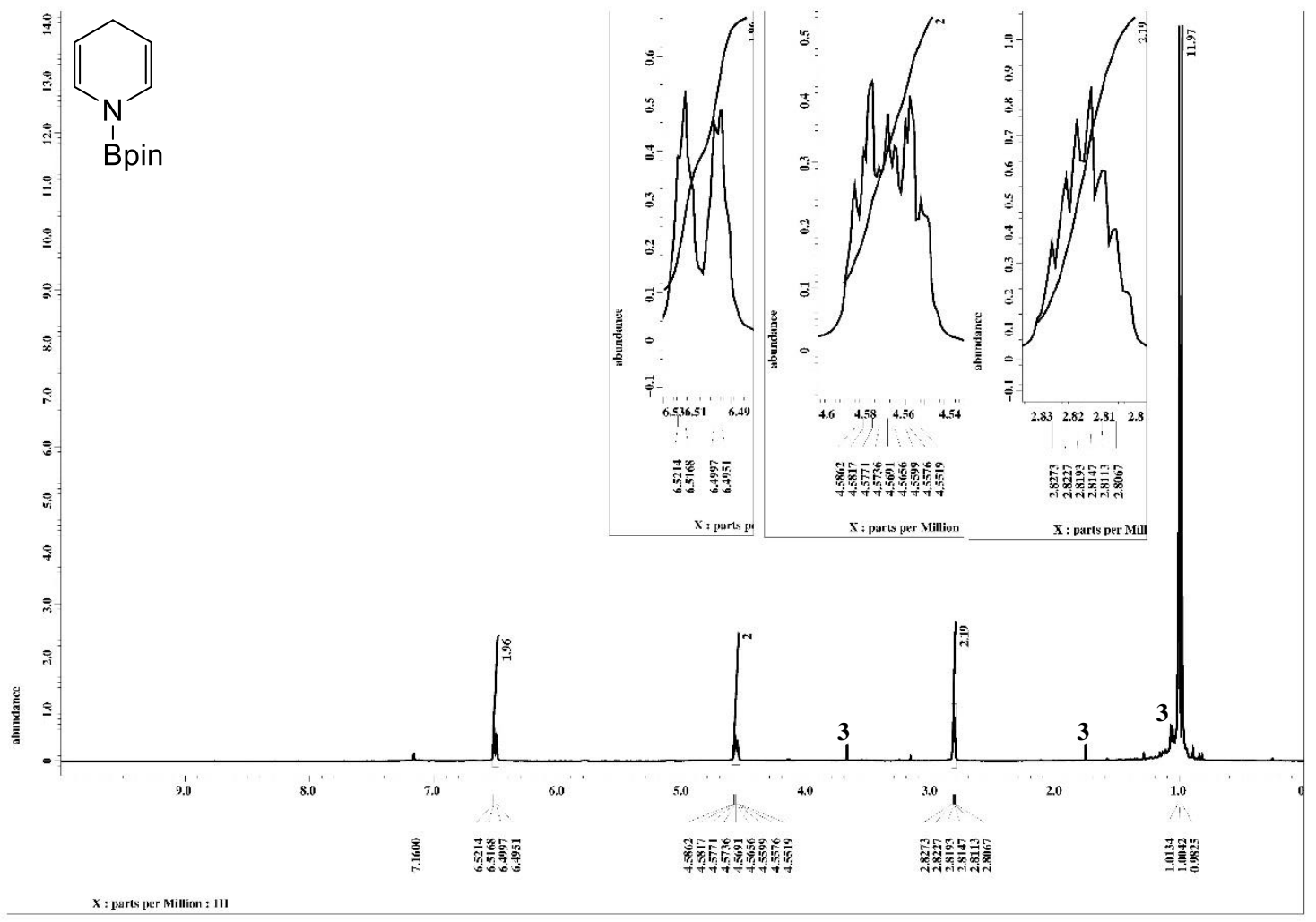

Figure S78. ${ }^{1} \mathrm{H}$ NMR spectrum of compound 9a (in $\mathrm{C}_{6} \mathrm{D}_{6}$ ).

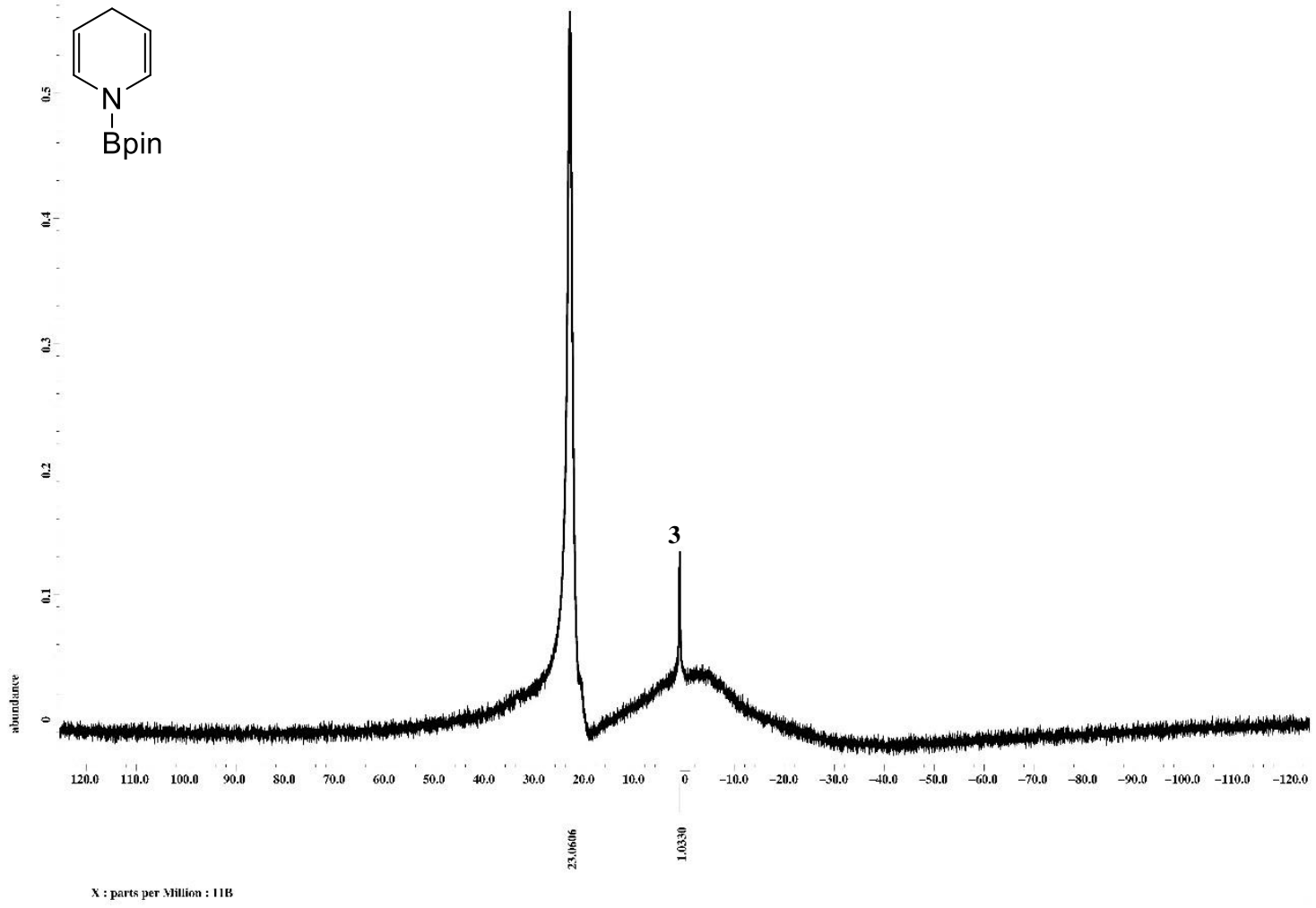

Figure S79. ${ }^{11} \mathrm{~B}\left\{{ }^{1} \mathrm{H}\right\}$ NMR spectrum of compound $9 \mathbf{a}$ (in $\mathrm{C}_{6} \mathrm{D}_{6}$ ). 


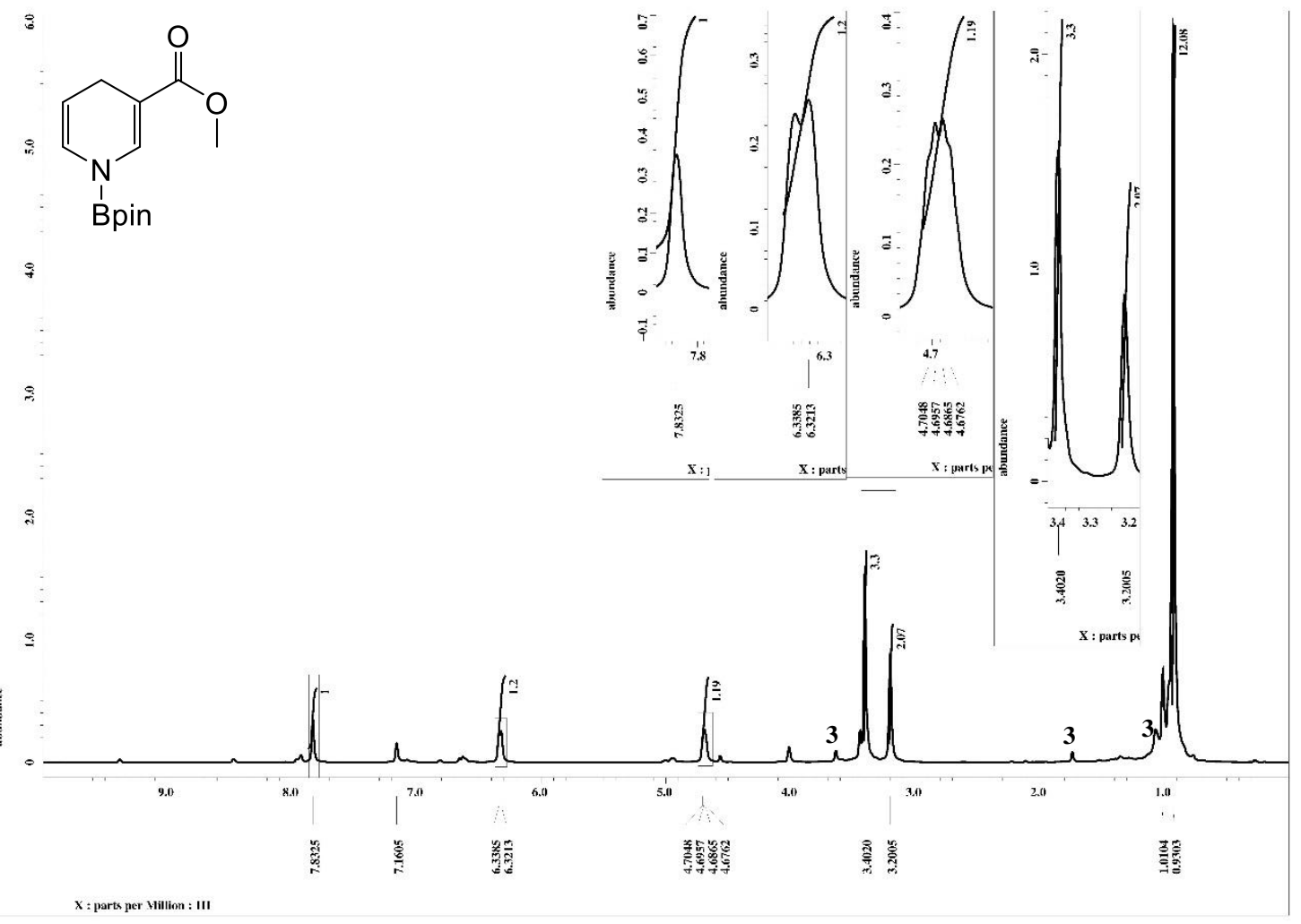

Figure S80. ${ }^{1} \mathrm{H}$ NMR spectrum of compound $9 b$ (in $\mathrm{C}_{6} \mathrm{D}_{6}$ ).

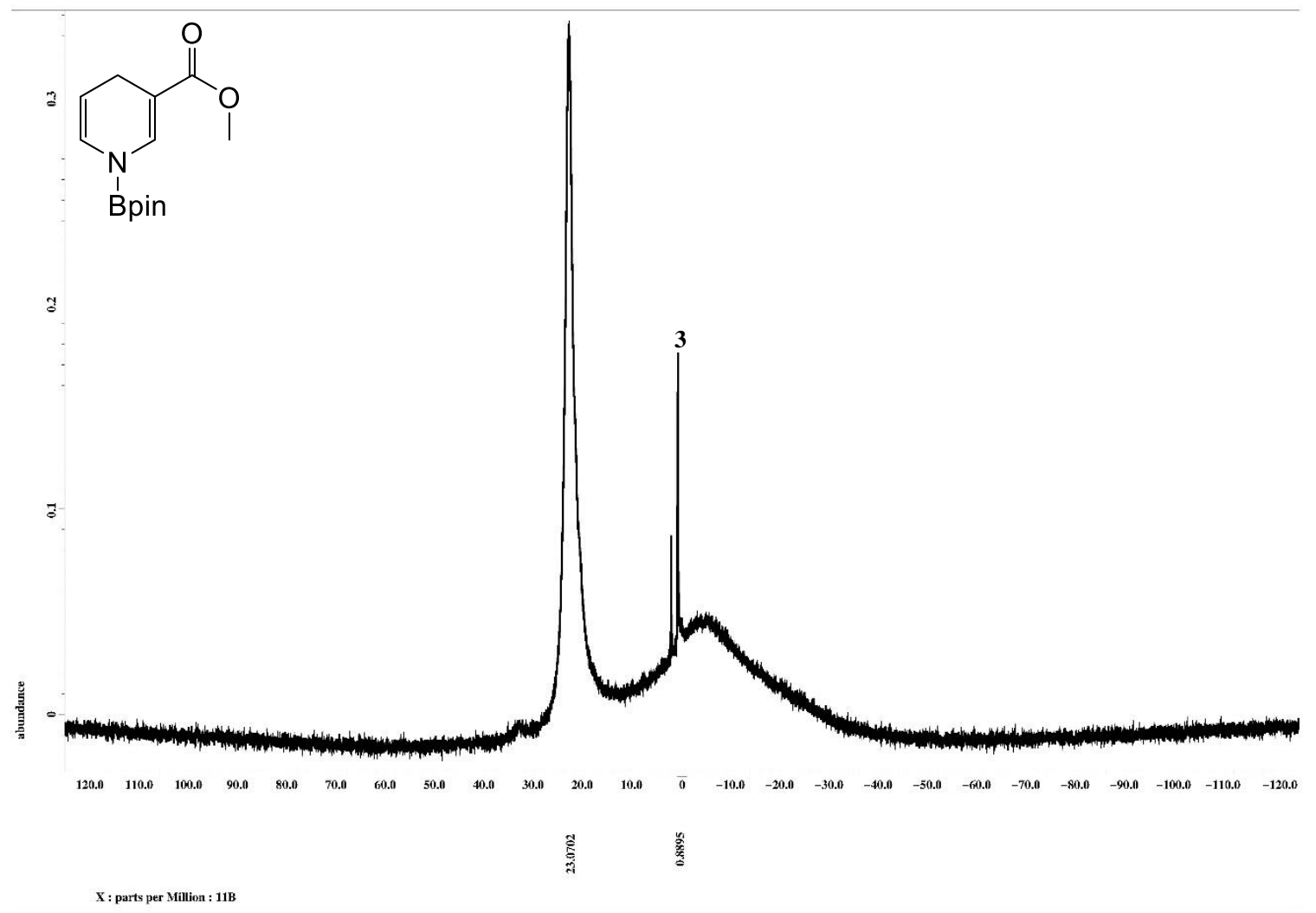

Figure S81. ${ }^{11} \mathrm{~B}\left\{{ }^{1} \mathrm{H}\right\}$ NMR spectrum of compound $9 \mathrm{~b}$ (in $\mathrm{C}_{6} \mathrm{D}_{6}$ ). 


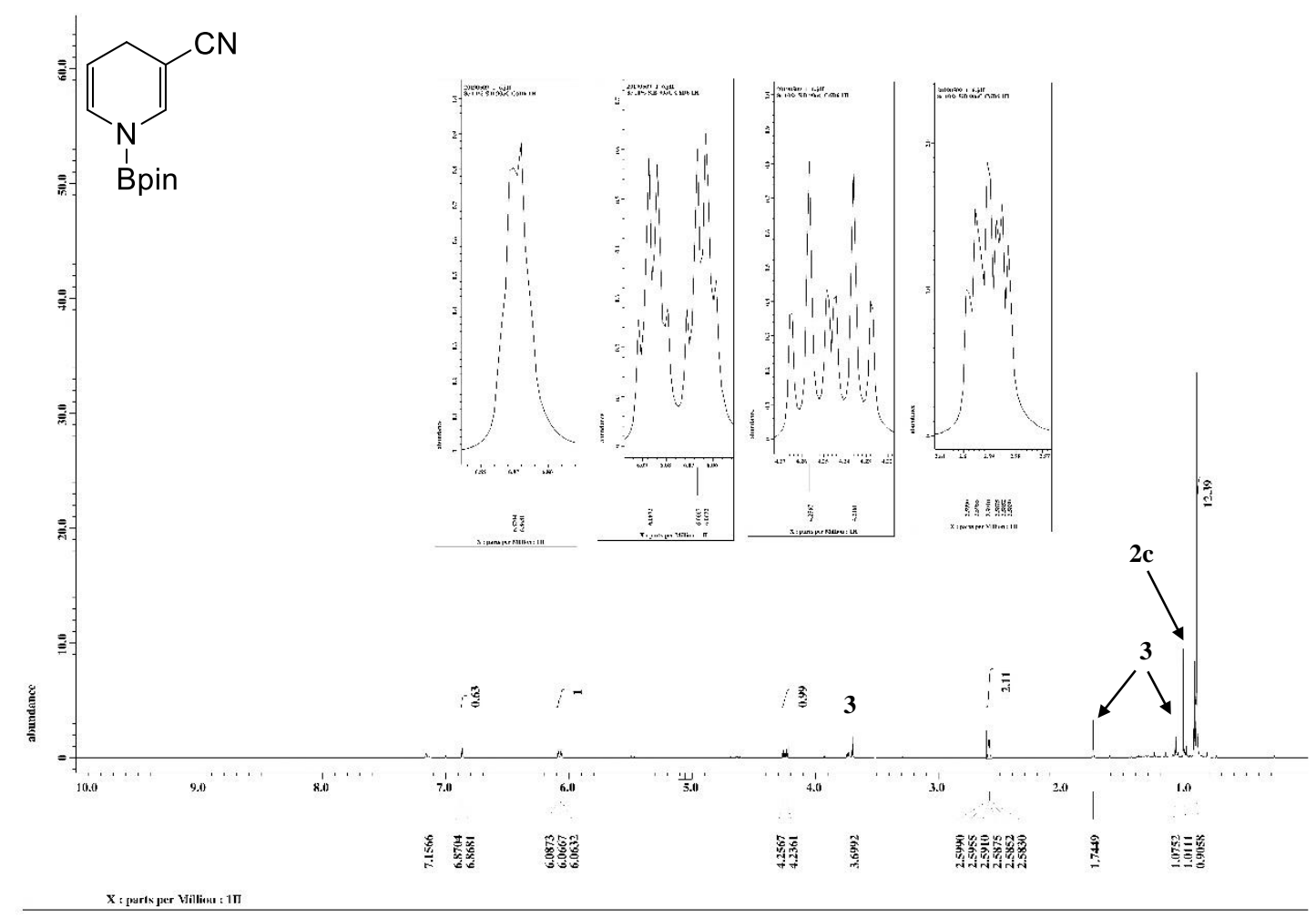

(a)

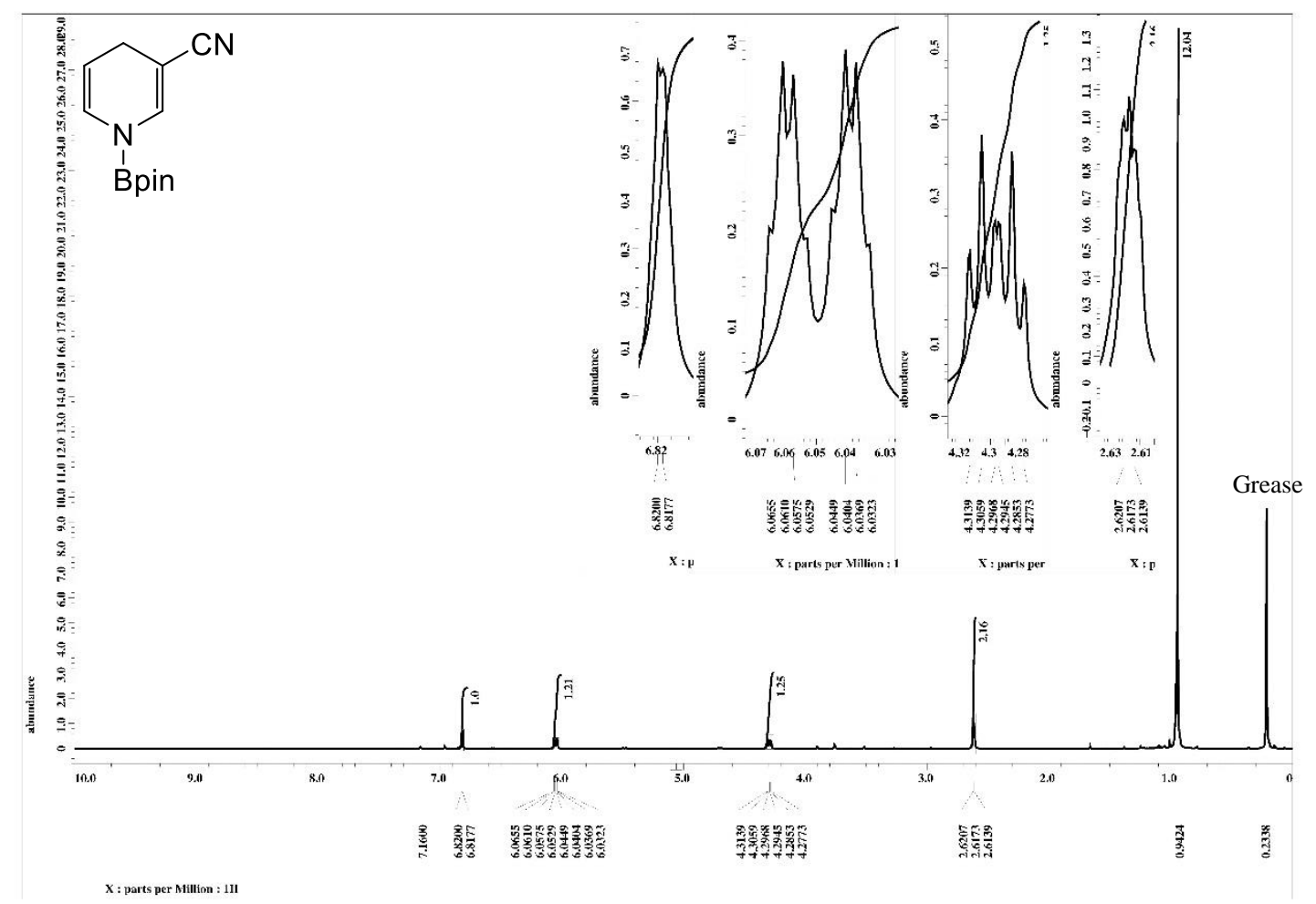

(b)

Figure S82. ${ }^{1} \mathrm{H}$ NMR spectrum of isolated compound $9 \mathrm{c}$ (in $\mathrm{C}_{6} \mathrm{D}_{6}$ ), (a) crude solution and (b) isolated compound. 


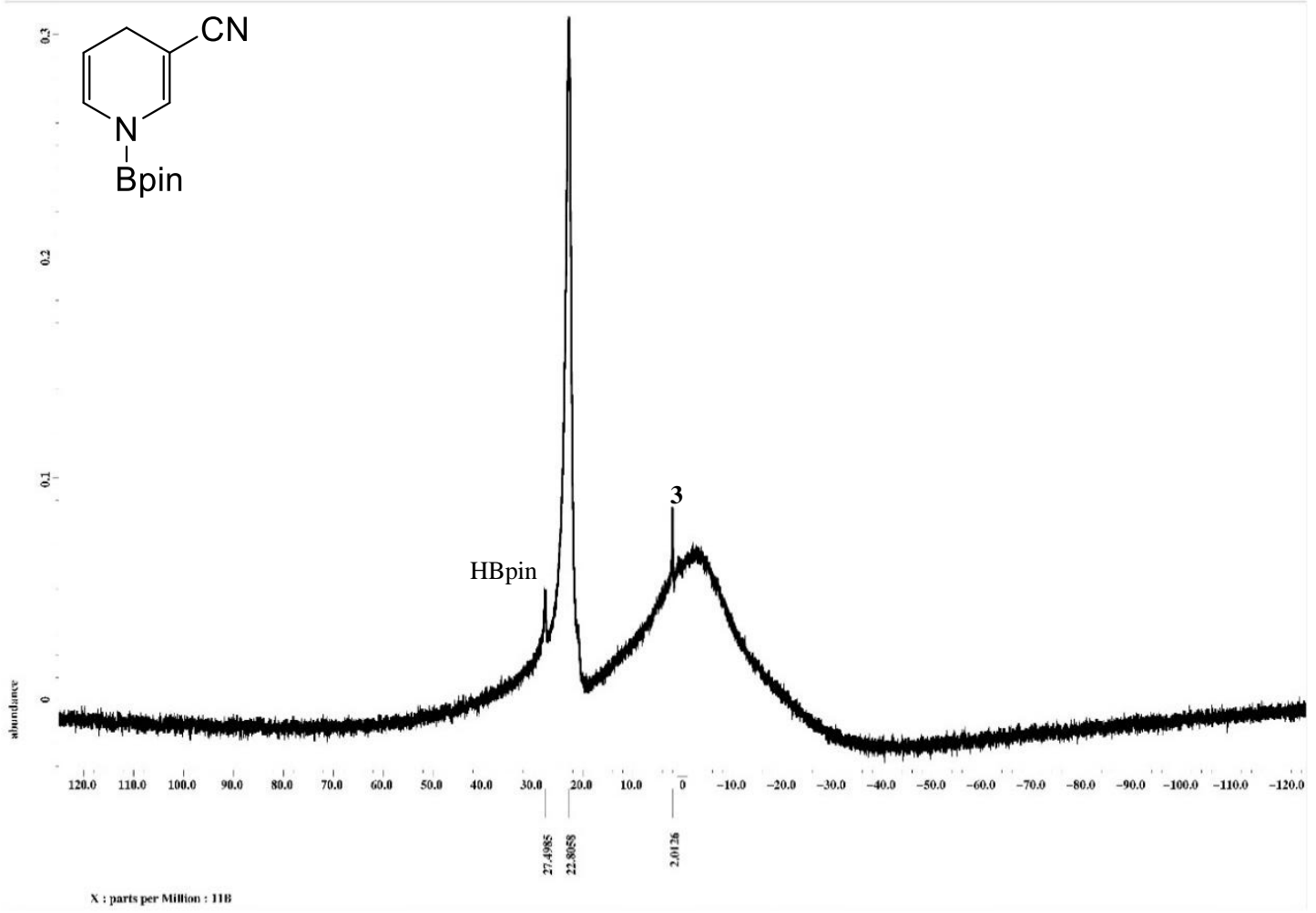

(a)

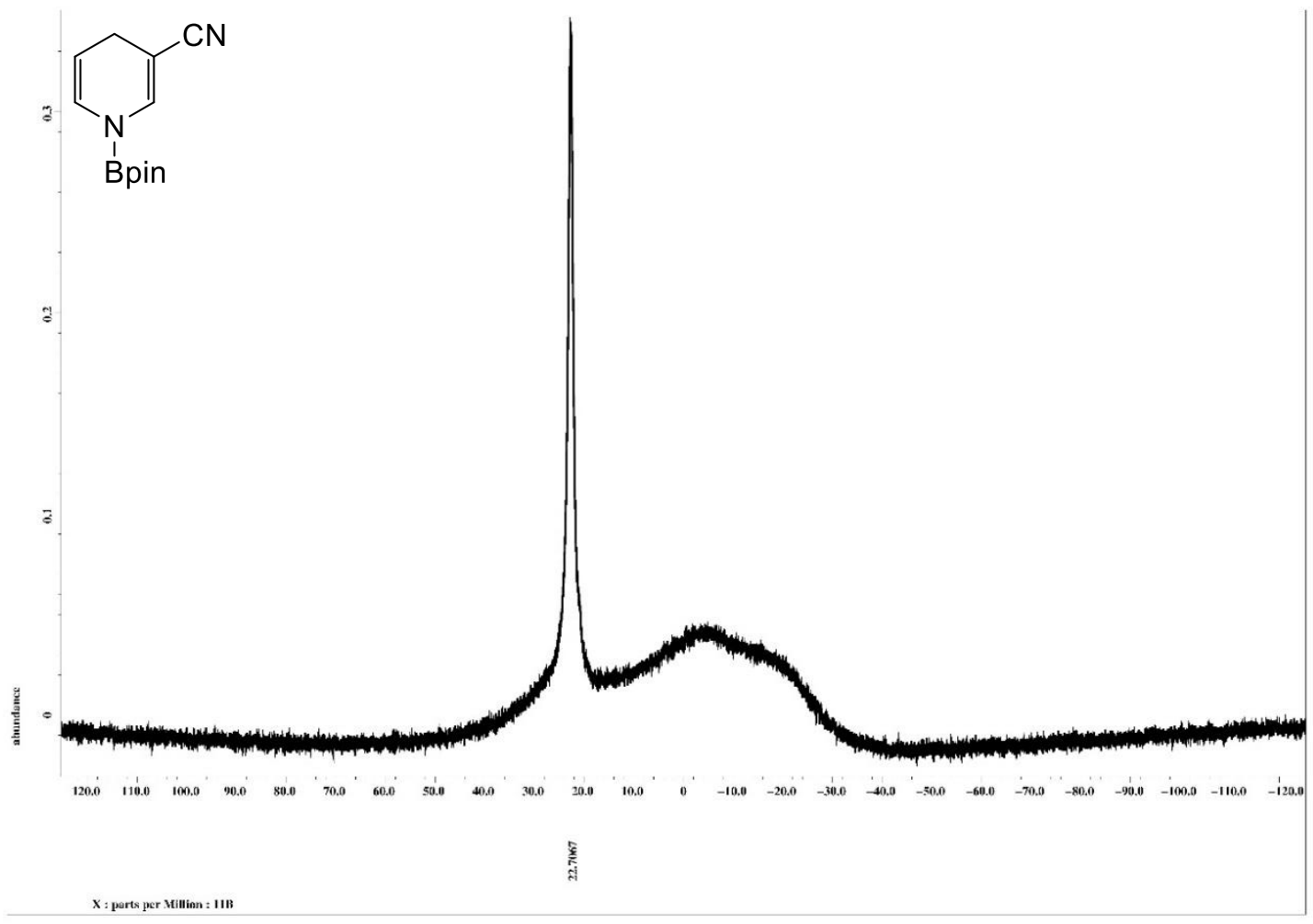

(b)

Figure S83. ${ }^{11} \mathrm{~B}\left\{{ }^{1} \mathrm{H}\right\}$ NMR spectrum of isolated compound 9c (in $\mathrm{C}_{6} \mathrm{D}_{6}$ ), (a) crude solution and (b) isolated compound. 


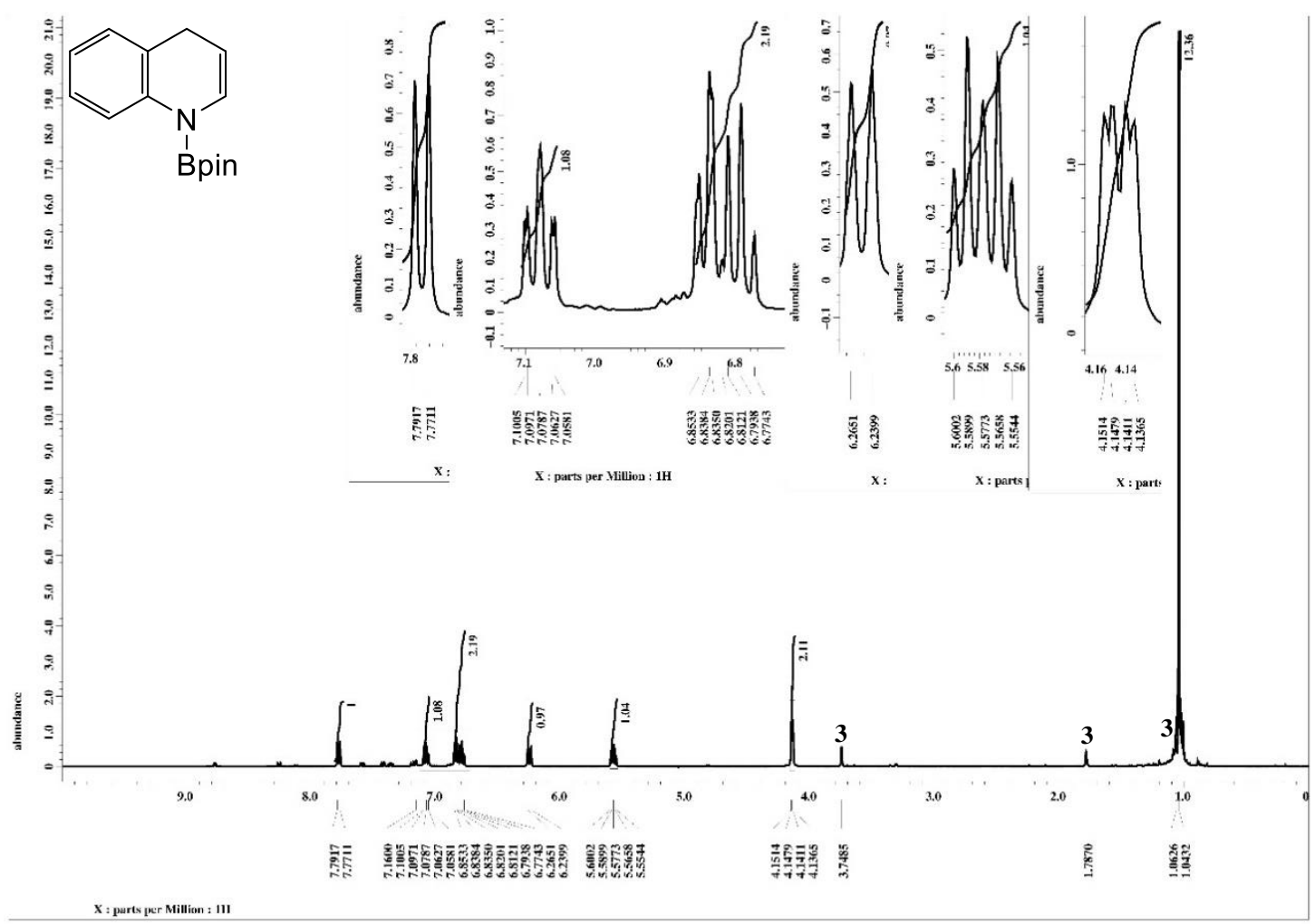

(a)

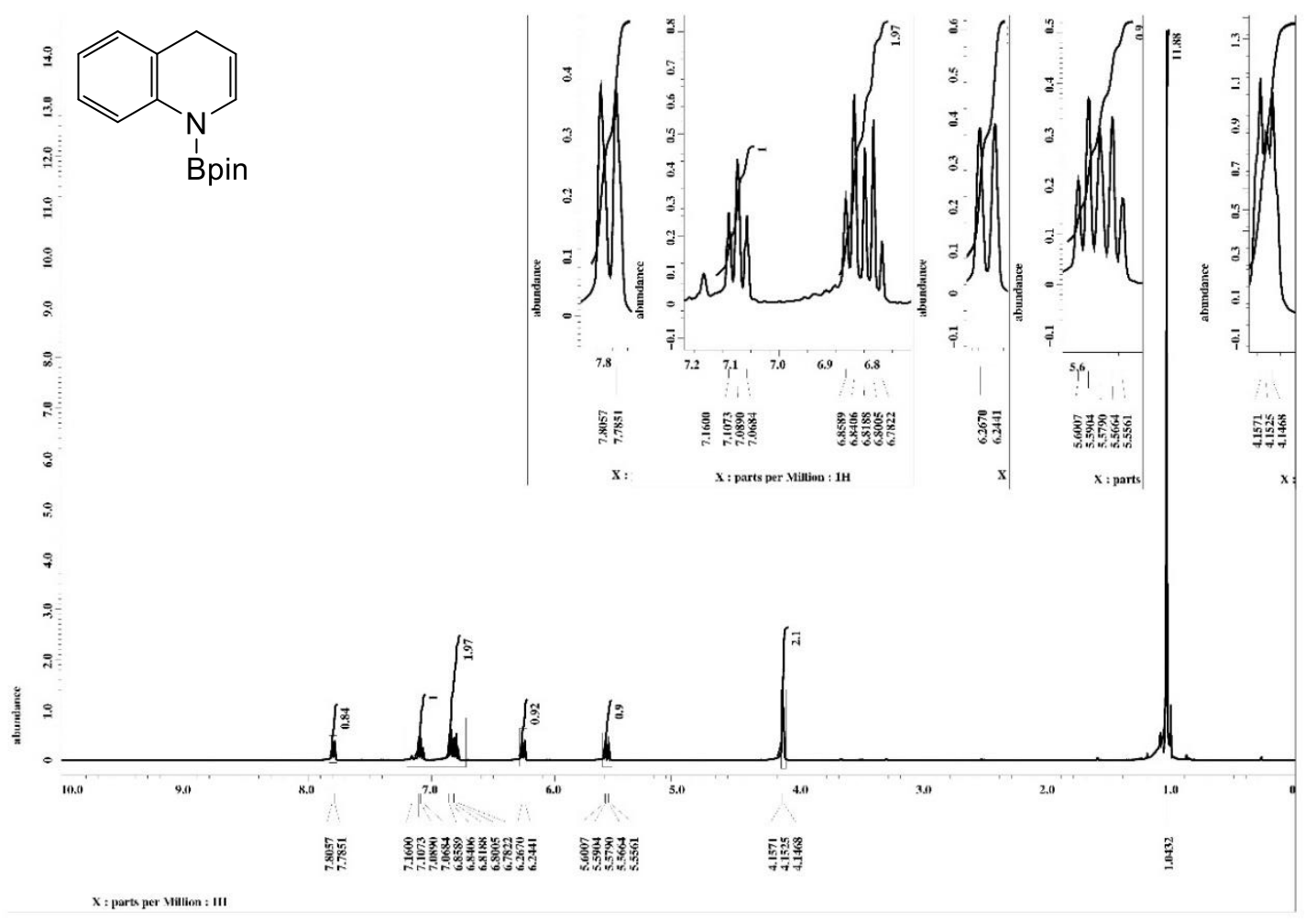

(b)

Figure S84. ${ }^{1} \mathrm{H}$ NMR spectrum of isolated compound 9d (in $\mathrm{C}_{6} \mathrm{D}_{6}$ ), (a) crude solution and (b) isolated compound. 


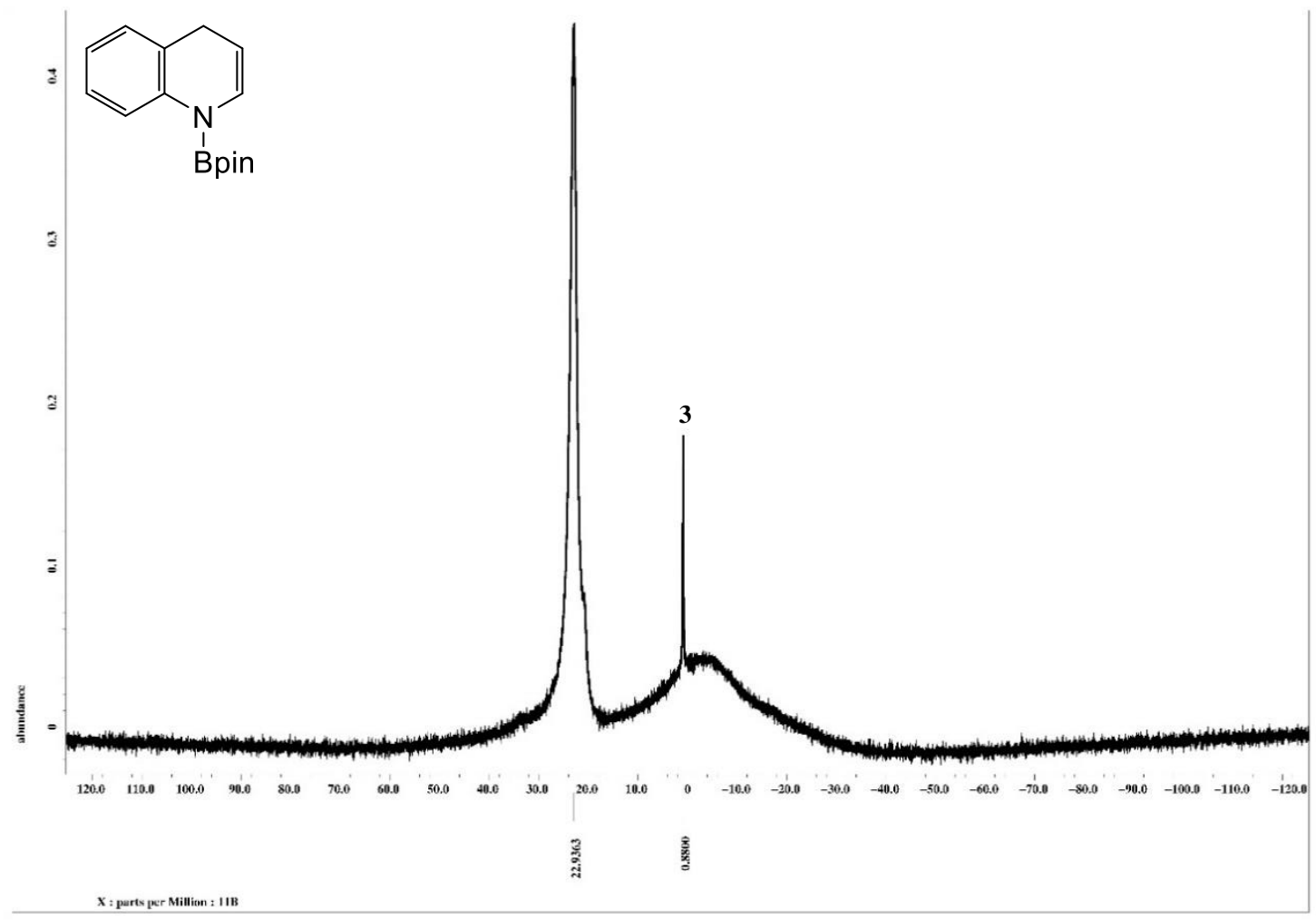

(a)

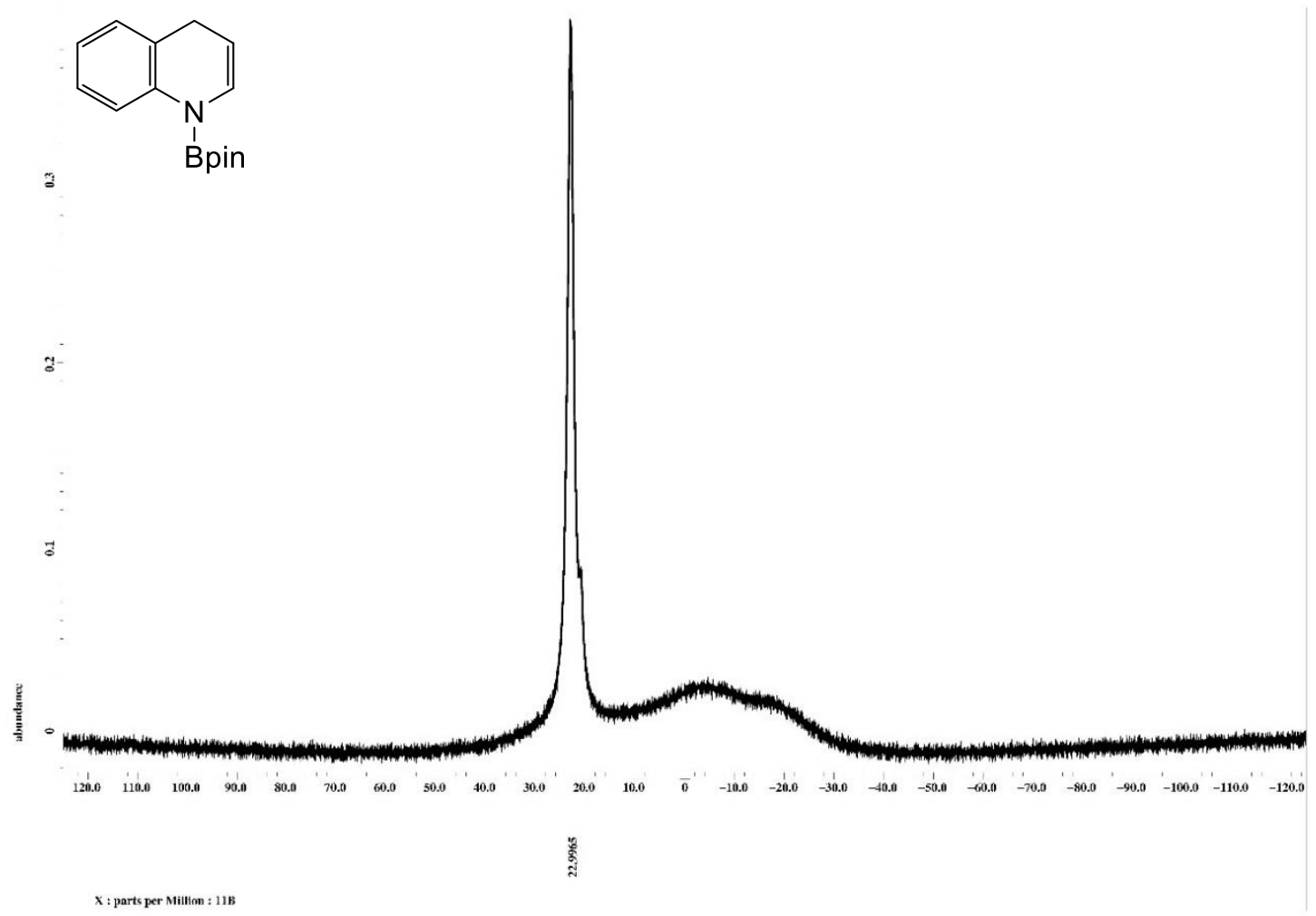

(b)

Figure S85. ${ }^{11} \mathrm{~B}\left\{{ }^{1} \mathrm{H}\right\}$ NMR spectrum of isolated compound 9d (in $\mathrm{C}_{6} \mathrm{D}_{6}$ ), (a) crude solution and (b) isolated compound.

Page S89 


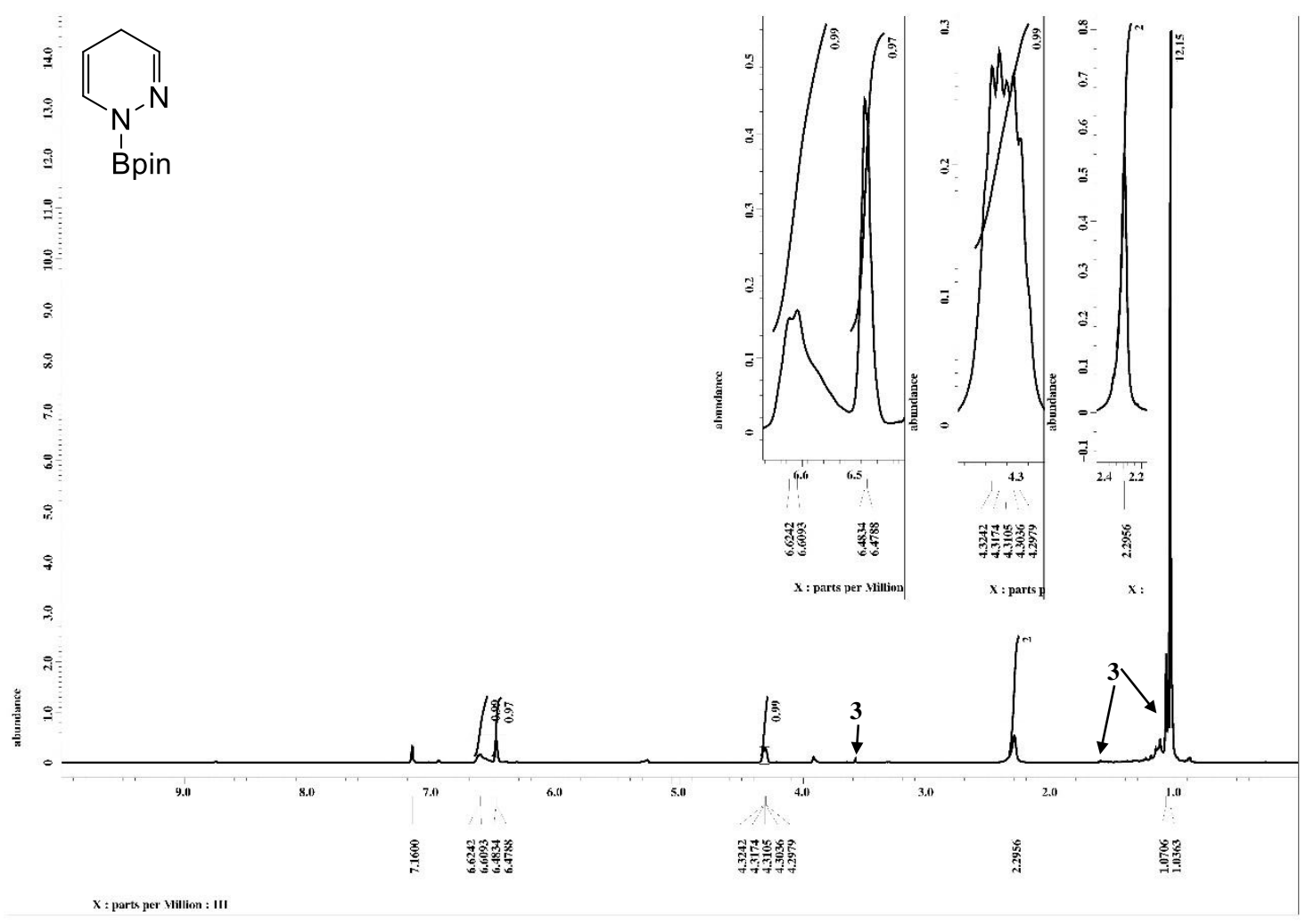

Figure S86. ${ }^{1} \mathrm{H}$ NMR spectrum of compound $9 \mathrm{e}$ (in $\mathrm{C}_{6} \mathrm{D}_{6}$ ).

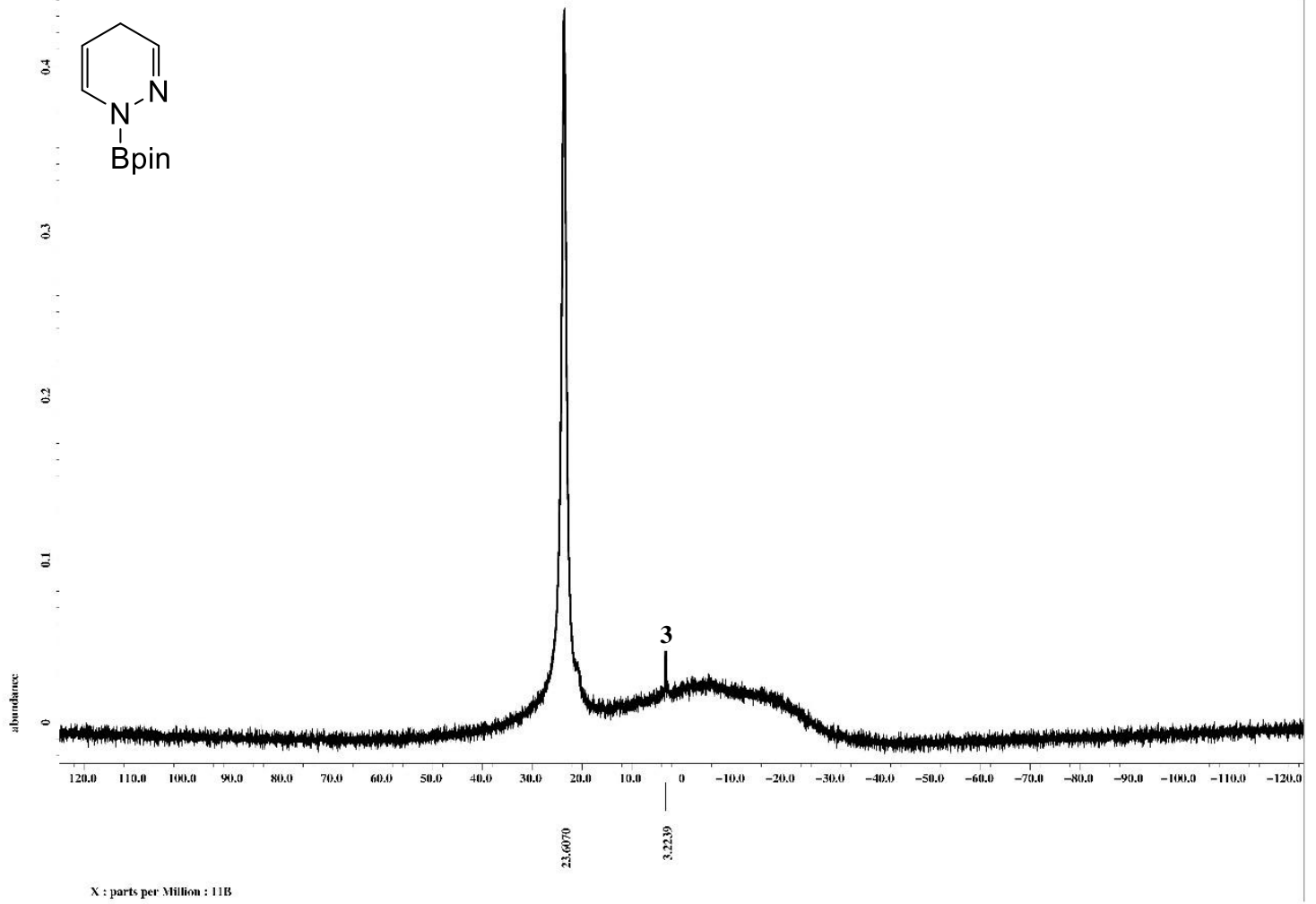

Figure S87. ${ }^{11} \mathrm{~B}\left\{{ }^{1} \mathrm{H}\right\}$ NMR spectrum of compound $9 \mathrm{e}$ (in $\left.\mathrm{C}_{6} \mathrm{D}_{6}\right)$. 


\section{S3. X-ray data collection and structural refinement}

The X-ray diffraction intensity data were measured at $100 \mathrm{~K}$ with a Bruker APEX II diffractometer equipped with a CCD detector, employing Mo K $\alpha$ radiation $(\lambda=0.71073 \AA)$, with the SMART suite of programs. ${ }^{\mathrm{S} 6}$ All data were processed and corrected for Lorentz and polarization effects with SAINT and for absorption effects with SADABS. ${ }^{\text {S6 }}$ Structural solution and refinement were carried out with the SHELXTL suite of programs. ${ }^{57}$ The structures were solved by direct methods to locate the heavy atoms, followed by difference maps for the light, non-hydrogen atoms. All non-hydrogen atoms were refined with anisotropic thermal

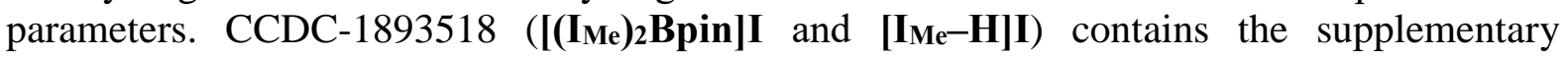
crystallographic data for this paper. The data can be obtained free of charge from the Cambridge Crystallography Data Center via www.ccdc.cam.ac.uk/data request/cif.

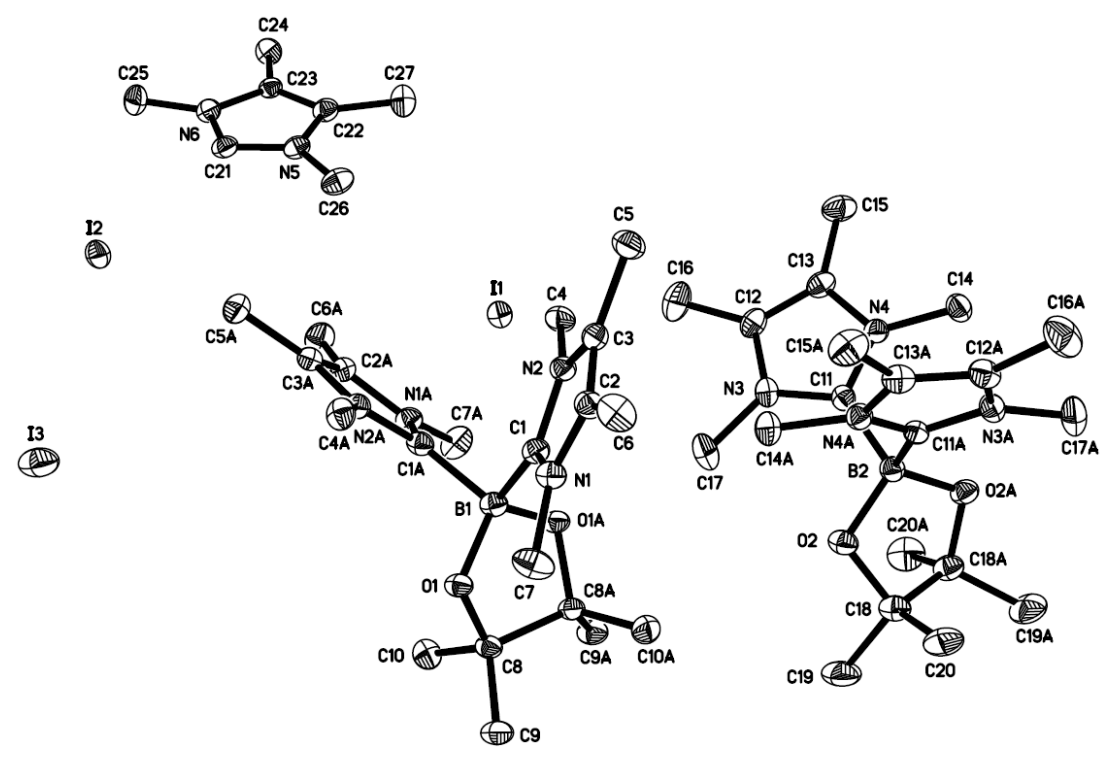

Figure S88. Molecular structure of [(IMe)2Bpin]I and [IMe-H]I (30\% ellipsoid probability). Hydrogen atoms are omitted for clarity. Selected bond lengths (A) and angles (deg): B1-C1 1.671(3), B2-C11 1.654(3); C1-B1-C1A 105.9(3), C11-B2-C11A 106.8(3), O1-B1-C1 111.23(10), O1-B1-C1A 110.79(11), O2-B2-C11 110.53(10), O2-B2-C11A 111.42(11). 


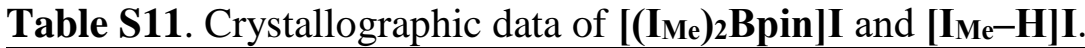

\begin{tabular}{|c|c|}
\hline & {$\left[\left(\mathbf{I}_{\mathbf{M e}}\right)_{2} \mathbf{B p i n}\right] \mathbf{I}$ and $\left[\mathbf{I}_{\mathbf{M e}}-\mathbf{H}\right] \mathbf{I}$} \\
\hline Formula & $\mathrm{C}_{23.50} \mathrm{H}_{42.50} \mathrm{BI}_{1.50} \mathrm{~N}_{5} \mathrm{O}_{2}$ \\
\hline Fw & 628.28 \\
\hline Color & Colourless \\
\hline cryst syst & Monoclinic \\
\hline space group & $C 12 / c 1$ \\
\hline$a(\AA)$ & $13.0847(6)$ \\
\hline$b(\AA)$ & $32.8113(16)$ \\
\hline$c(\AA)$ & $13.7965(7)$ \\
\hline$\alpha(\mathrm{deg})$ & 90 \\
\hline$\beta(\mathrm{deg})$ & $106.2955(18)$ \\
\hline$\gamma(\mathrm{deg})$ & 90 \\
\hline$V\left(\AA^{3}\right)$ & $5685.2(5)$ \\
\hline$Z$ & 8 \\
\hline$d_{\text {calcd }}\left(\mathrm{g} \mathrm{cm}^{-3}\right)$ & 1.468 \\
\hline$\mu\left(\mathrm{mm}^{-1}\right)$ & 1.697 \\
\hline$F(000)$ & 2552 \\
\hline cryst size $(\mathrm{mm})$ & $0.120 \times 0.140 \times 0.200$ \\
\hline $2 \theta$ range (deg) & 2.47 to 35.01 \\
\hline index range & $\begin{array}{l}-21 \leq h \leq 20 \\
-52 \leq k \leq 52 \\
-22 \leq l \leq 21\end{array}$ \\
\hline no. of rflns collected & 63404 \\
\hline no. of indep rflns & 12505 \\
\hline$R 1, w R 2(I>2 \sigma(I))$ & $0.0451,0.0799$ \\
\hline$R 1, w R 2$ (all data) & $0.0868,0.0967$ \\
\hline goodness of fit, $F^{2}$ & 1.022 \\
\hline no. of data/restraints/params & 12505 / 84 / 387 \\
\hline largest diff peak and hole, $\mathrm{e} \AA^{-3}$ & 1.288 and -1.574 \\
\hline
\end{tabular}




\section{S5. Theoretical Studies}

Geometry optimizations were fully performed without any symmetry restriction by using M062X/def2-TZVP level of theory as implemented in the Gaussian 09 package of programs. ${ }^{\mathrm{S} 8}$ The vibrational frequency calculations were performed to establish the nature of stationary points. The local minima were confirmed by zero imaginary frequencies, and transition states were characterized by an imaginary frequency. The thermodynamic corrections are obtained by using "temperature" keyword in Gaussian $09^{\mathrm{S} 8}$ at M06-2X/def2-TZVP.

List of abbreviation used in theoretical studies.

\begin{tabular}{|c|c|}
\hline Abbreviation & Definition \\
\hline Cpd & Compound \\
HBpin & Pinacolborane \\
Int & Intermediate \\
$\mathrm{I}_{\mathrm{Me}}$ & $: \mathrm{C}\{\mathrm{N}(\mathrm{Me}) \mathrm{C}(\mathrm{Me})\}_{2}$ \\
$\mathrm{TS}$ & Transition state \\
\hline
\end{tabular}

List of colour coded atom used in optimised structures

\begin{tabular}{|c|c|}
\hline Color & Definition \\
\hline White & Hydrogen \\
Black/ Grey & Carbon \\
Blue & Nitrogen \\
Yellow & Silicon \\
Green & Boron \\
Red & Oxygen \\
Purple & Iodine \\
\hline
\end{tabular}




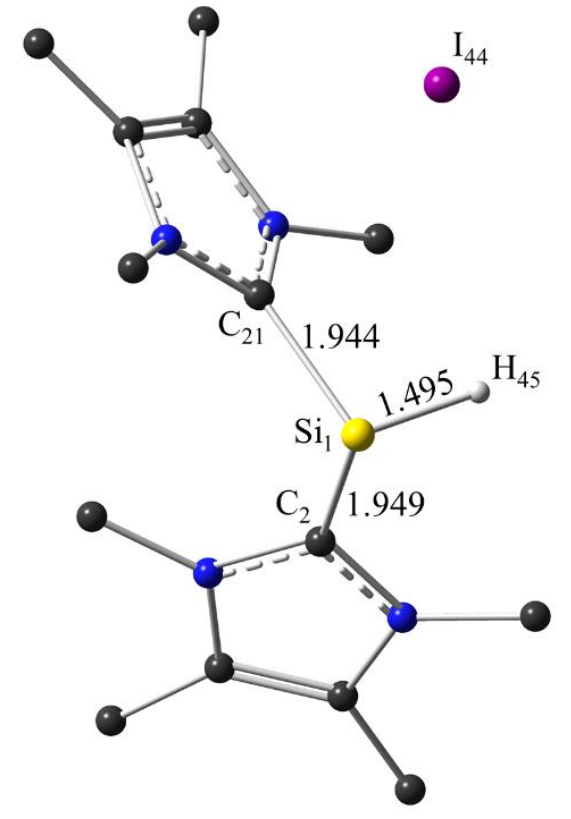

Compound 1

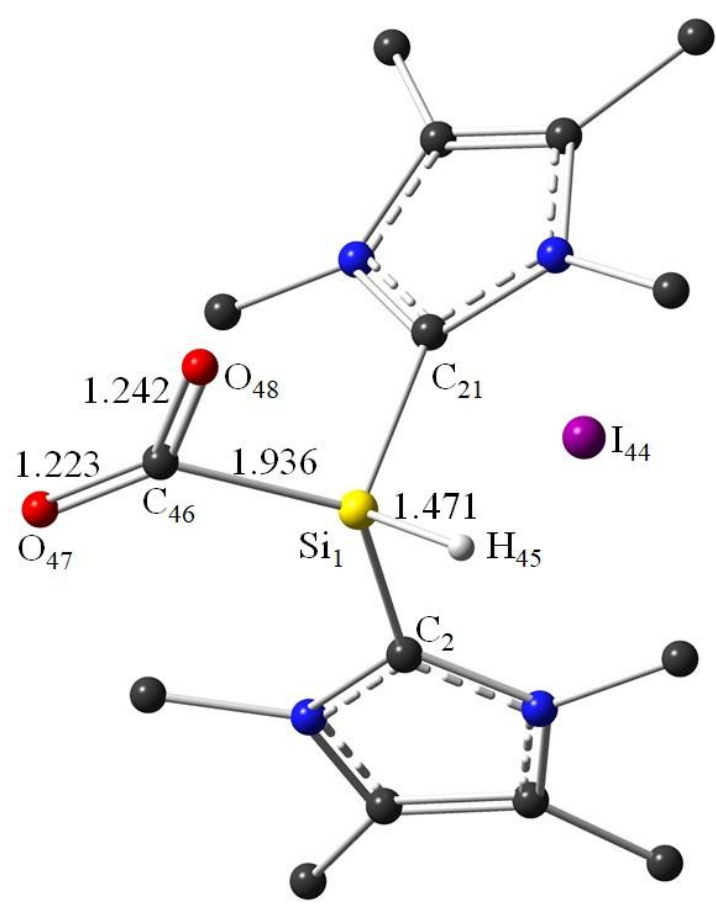

Int01

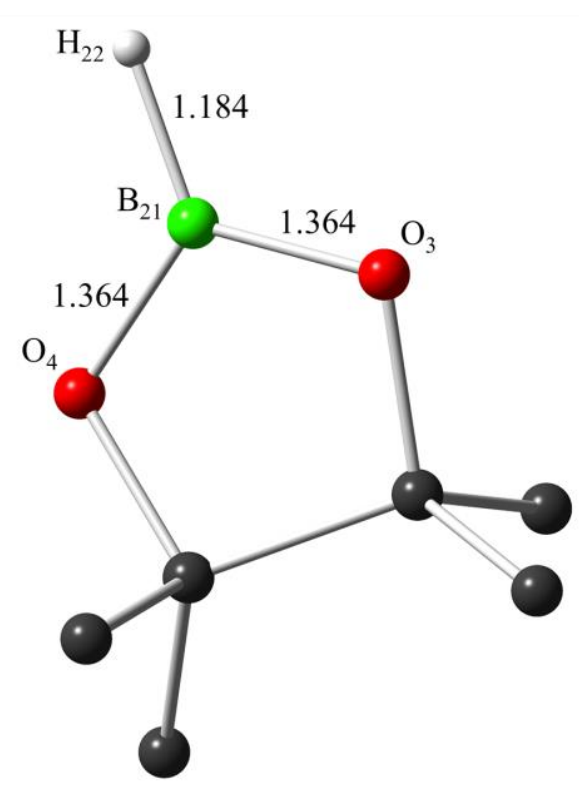

HBpin

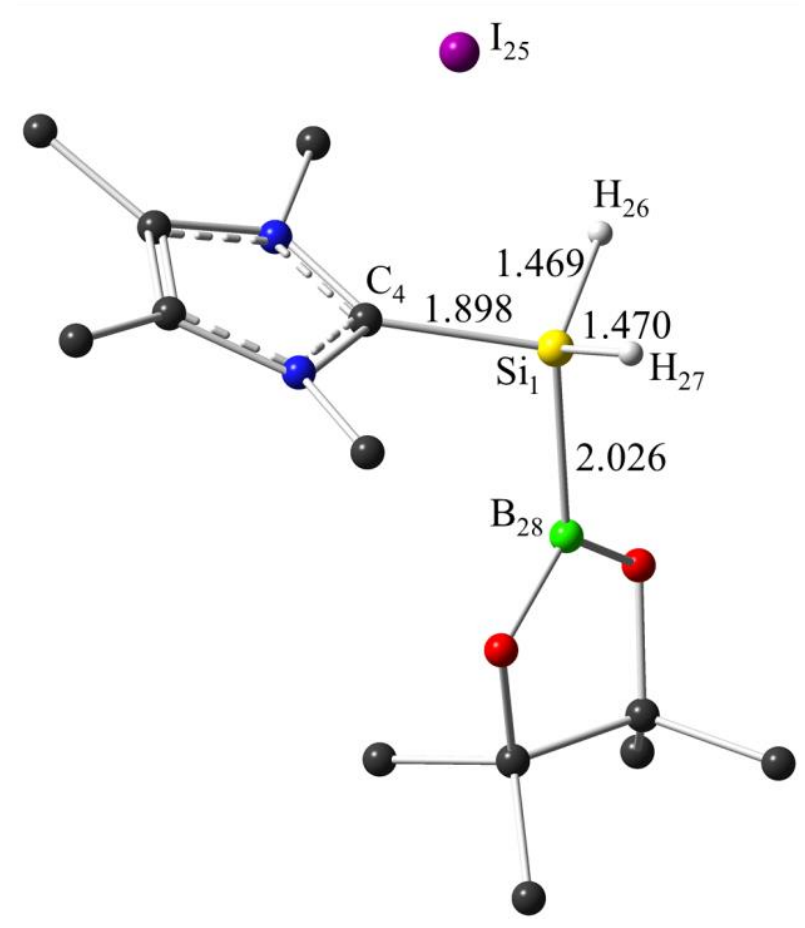

Int02

Figure S89. Optimised geometries of the minimal and transition states at M06-2X/def2-TZVP level of theory. Hydrogen atoms are omitted for clarity. The bond lengths displayed are measured in Angstroms ( $)$. 


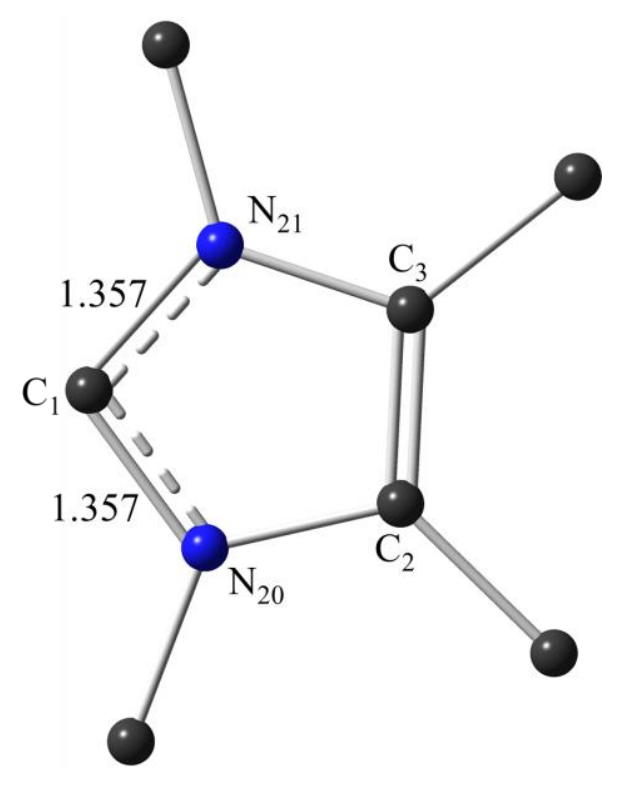

$\mathrm{I}_{\mathrm{Me}}$

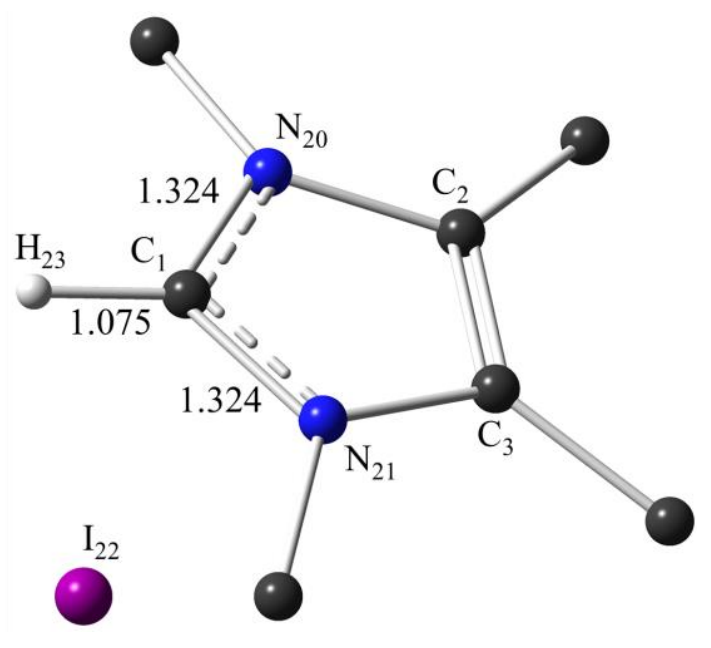

$$
\left[\mathrm{I}_{\mathrm{Me}}-\mathrm{H}\right] \mathrm{I}
$$

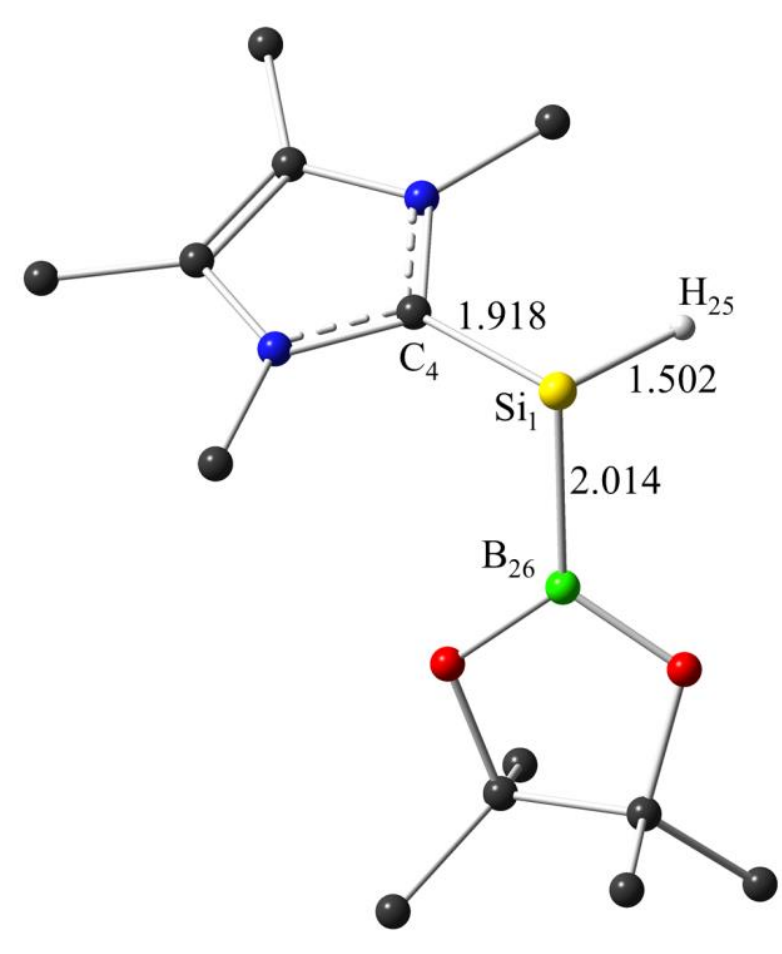

Int03

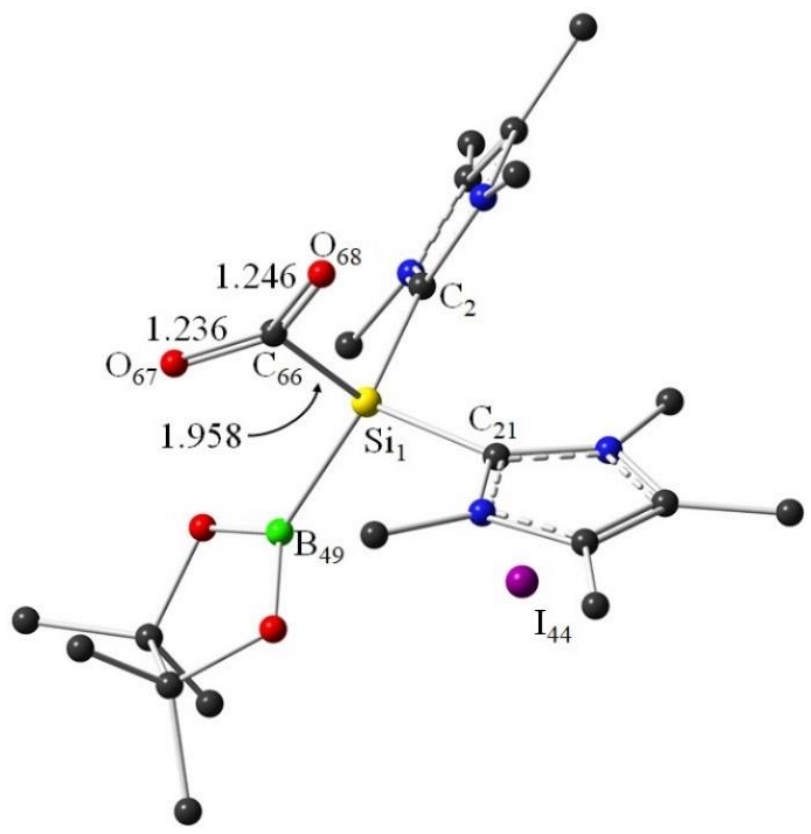

Int04

Figure S89 (continued). Optimised geometries of the minimal and transition states at M062X/def2-TZVP level of theory. Hydrogen atoms are omitted for clarity. The bond lengths displayed are measured in Angstroms $(\AA)$. 


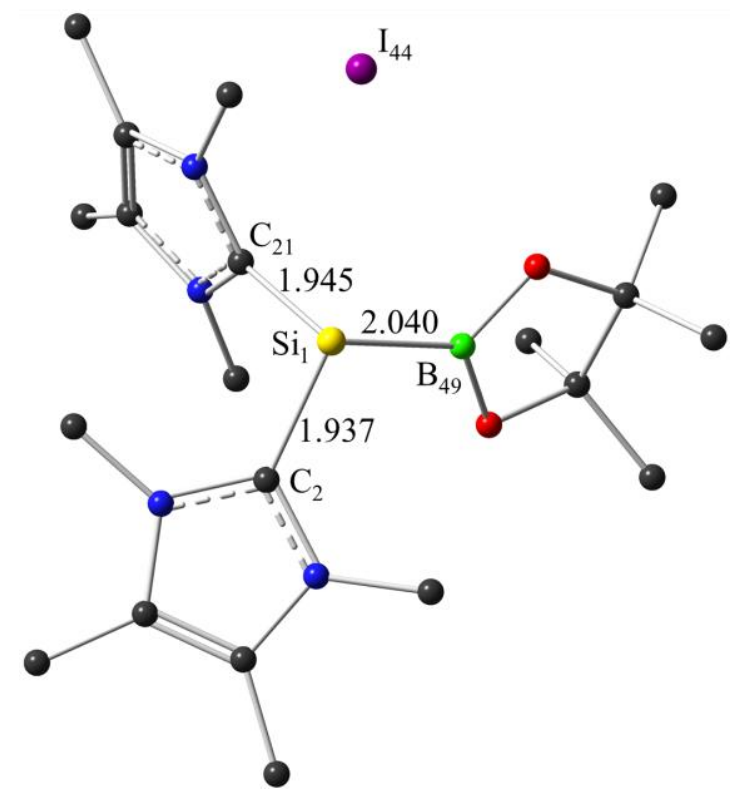

Compound 3

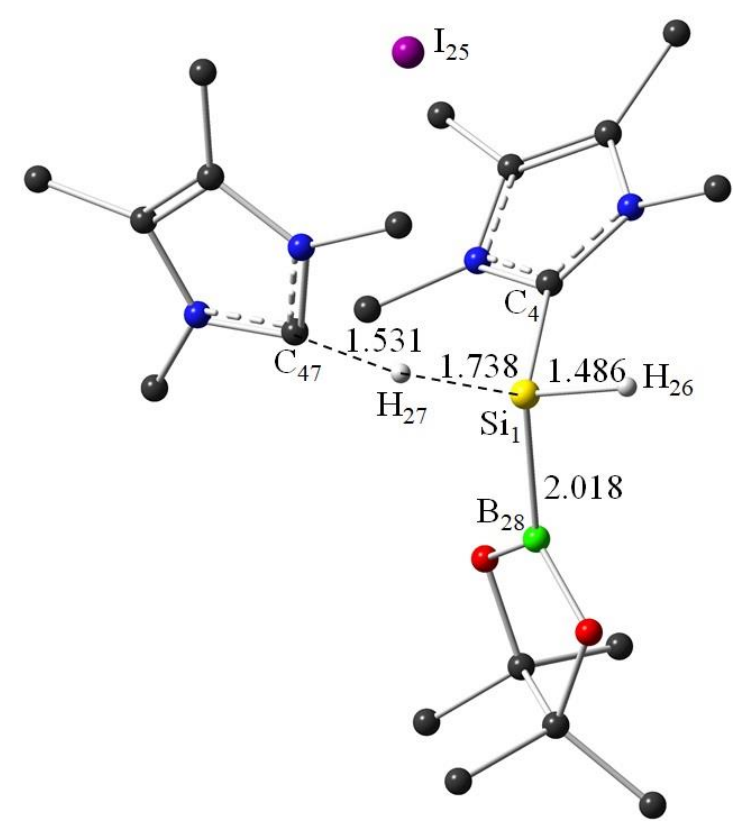

$\mathrm{TS} 02$

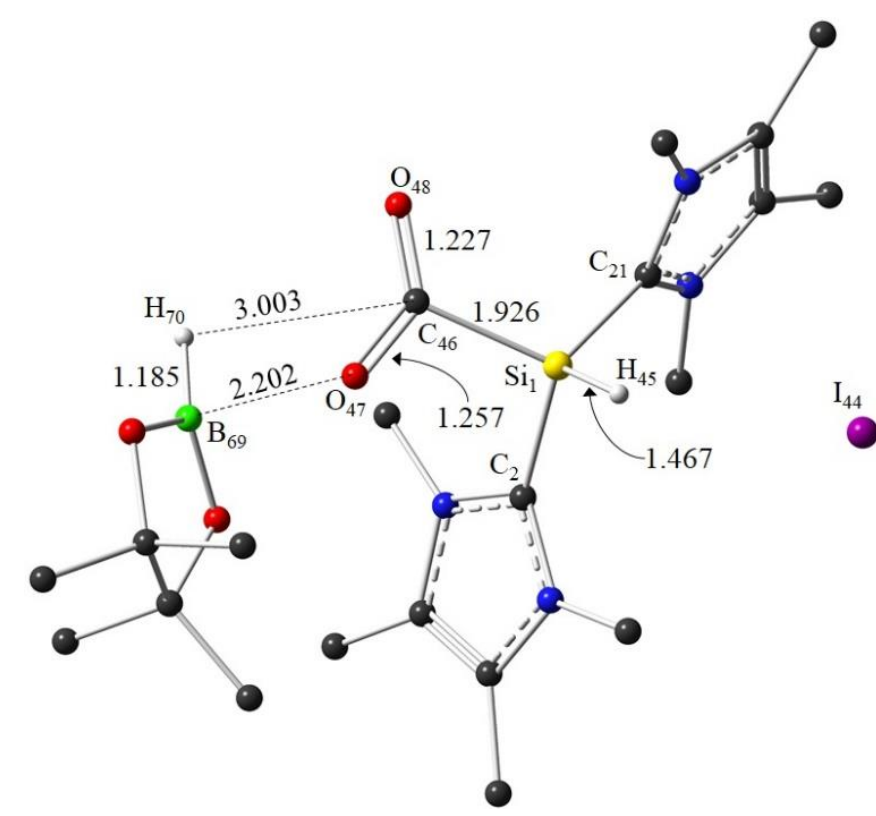

TS01

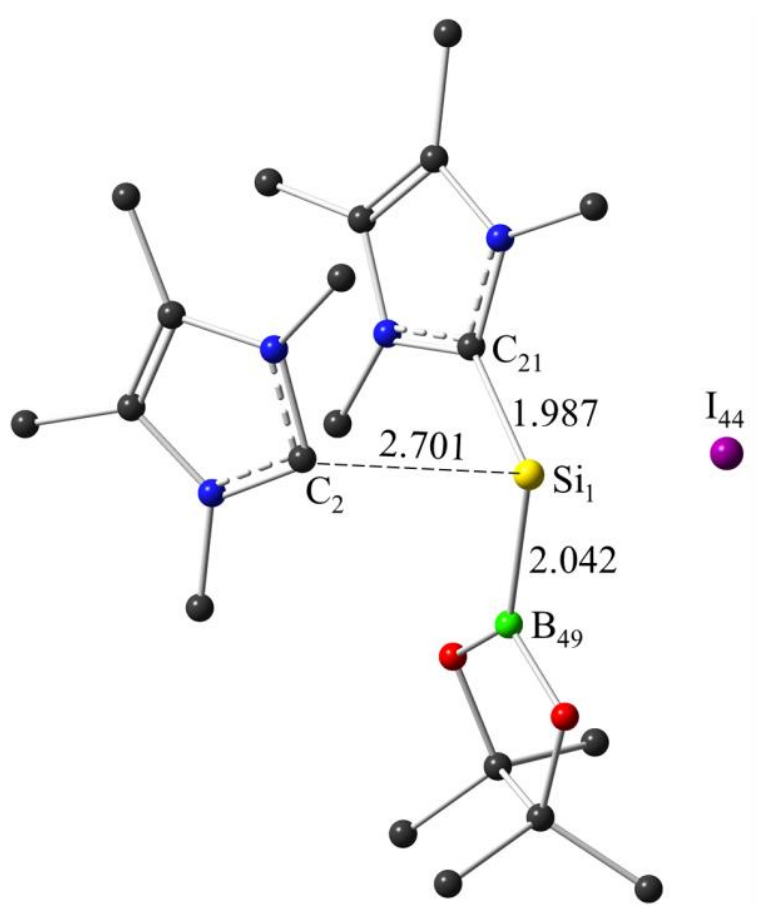

TS03

Figure S89 (continued). Optimised geometries of the minimal and transition states at M062X/def2-TZVP level of theory. Hydrogen atoms are omitted for clarity. The bond lengths displayed are measured in Angstroms $(\AA)$. 


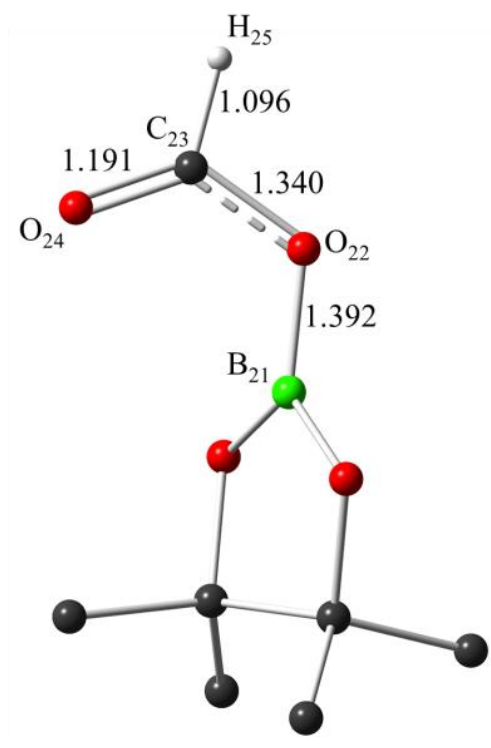

Compound 2a

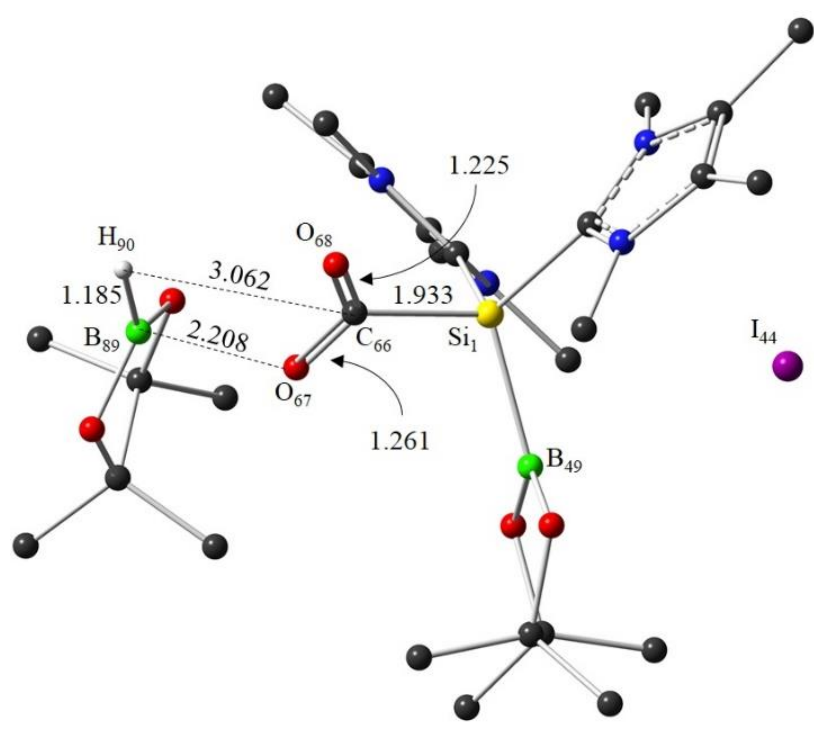

TS04

Figure S89 (continued). Optimised geometries of the minimal and transition states at M062X/def2-TZVP level of theory. Hydrogen atoms are omitted for clarity. The bond lengths displayed are measured in Angstroms ( $⿱$ ) $)$.

Table S12. The theoretical calculated ${ }^{29} \mathrm{Si}$ NMR chemical shift (ppm) in solution $\left(\mathrm{C}_{6} \mathrm{D}_{6}\right)$ using TMS as the reference at B3LYP/6-311 $1^{++} \mathrm{G}(2 \mathrm{df}, 2 \mathrm{pd}) / / \mathrm{B} 3 \mathrm{LYP} / 6-31 \mathrm{G} * *$ level of theory.

$\operatorname{System}(\mathrm{X}) \quad{ }^{29} \mathrm{Si}$ NMR chemical shift ${ }^{\mathrm{a}}$

Compound 3

$-94.0$

${ }^{\mathrm{a}} \delta\left({ }^{29} \mathrm{Si}_{\mathrm{x}}\right)=\sigma\left({ }^{29} \mathrm{Si} \mathrm{TMS}_{\mathrm{TM}}\right)-\sigma\left({ }^{29} \mathrm{Si}_{\mathrm{x}}\right) .{ }^{\mathrm{S} 9}$ 


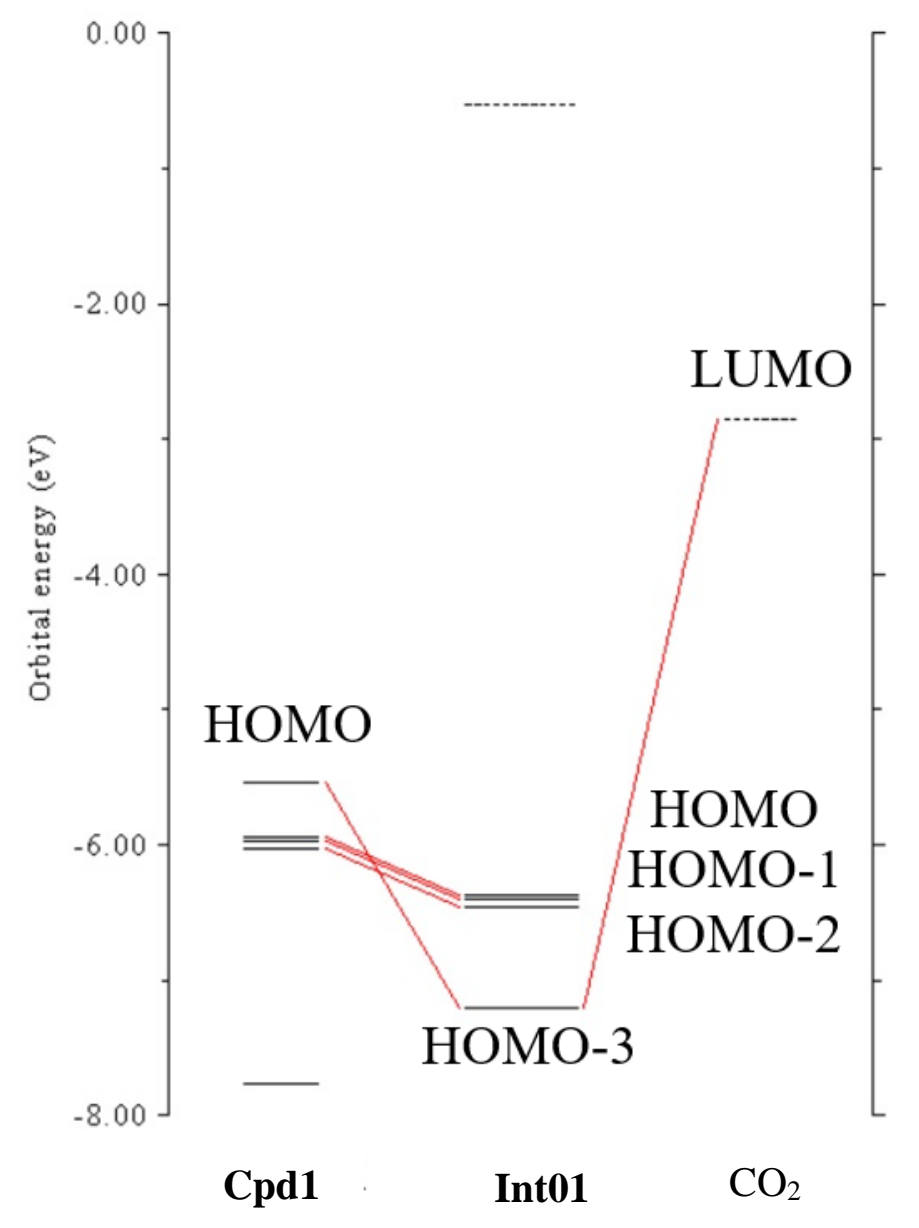

Figure S90. Orbital interaction diagram of compound 1 and $\mathrm{CO}_{2}$ at $\mathrm{M} 06-2 \mathrm{X} /$ def2-TZVP level of theory. ${ }^{\text {S10 }}$

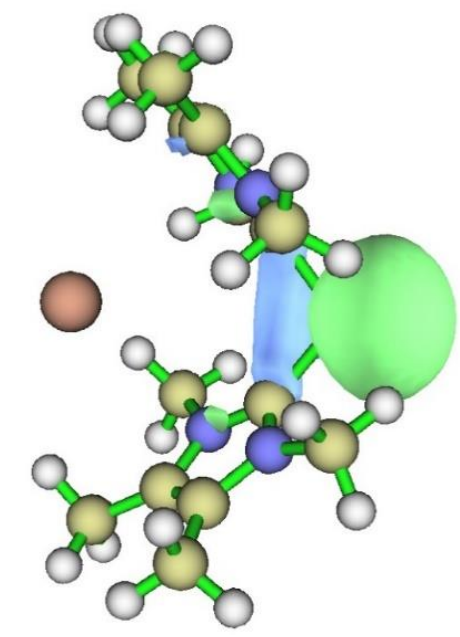

HOMO of compound 1

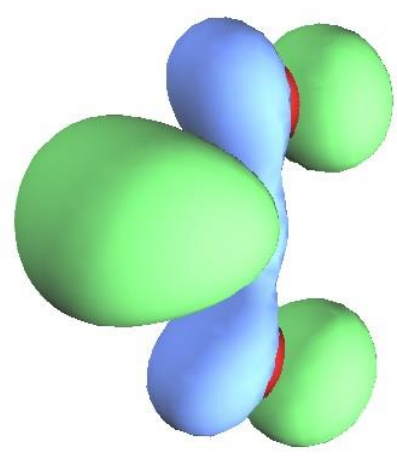

LUMO of $\mathrm{CO}_{2}$

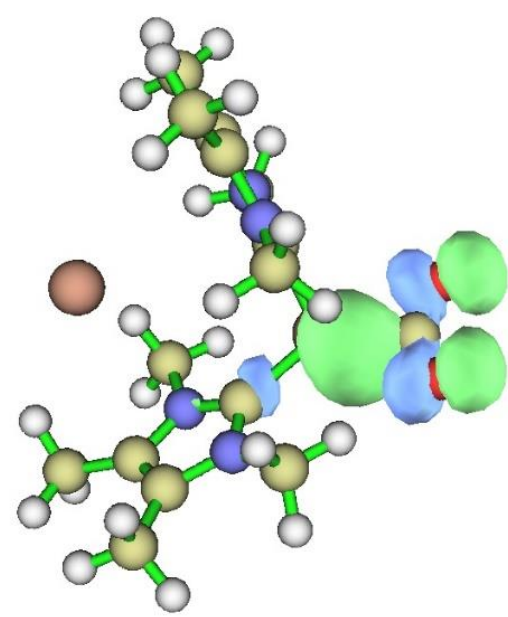

HOMO-3 of Int01

Figure S91. Molecular orbitals of compound 1, $\mathrm{CO}_{2}$ and Int01 at M06-2X/def2-TZVP level of theory. $\mathrm{S} 10$ 
Table S13. Natural Bond Order (NBO) of Int01 at M06-2X/def2-TZVP level of theory. ${ }^{\mathrm{S} 10}$

Contribution

Orbital

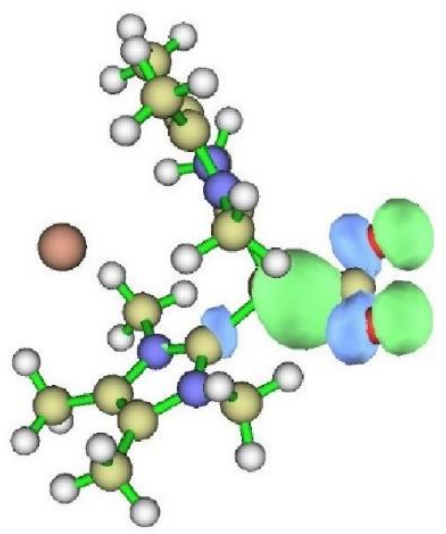

Occ.

from atoms to the

orb
Atomic orbitals

$\mathrm{Si}: 31.75 \% \mathrm{~s}+$

1.96

$\begin{array}{cc}45.68 \% \mathrm{Si}+ & 67.93 \% \mathrm{p} \\ 54.32 \% \mathrm{C} & \text { C: } 27.75 \% \mathrm{~s}+ \\ & 72.00 \% \mathrm{p}\end{array}$




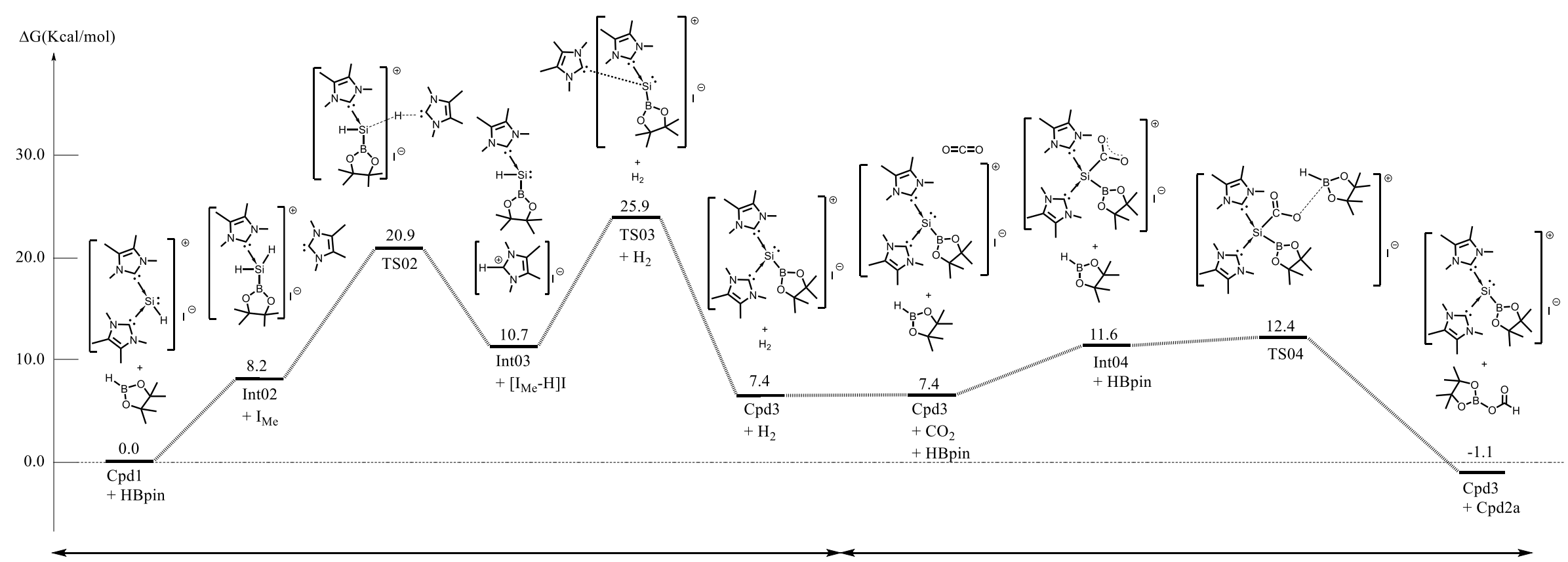

Formation of compound 3

Catalysis using compound 3

The mechanism for the formation of $\mathbf{3}$ is proposed. Compound $\mathbf{1}$ undergoes an oxidative addition with HBpin to form Int02, along with the displacement of $\mathrm{I}_{\mathrm{Me}}$. Similar oxidative addition of HBpin with a silylene was reported. ${ }^{\mathrm{S} 11} \mathrm{I}_{\mathrm{Me}}$ then reduces Int02 to form Int03 and [I $\left.\mathrm{I}_{\mathrm{Me}}-\mathrm{H}\right] \mathrm{I}$. Similar reduction was reported by Roesky et al. that NHC, $\left[: \mathrm{C}\{\mathrm{N}(\mathrm{Ar}) \mathrm{C}(\mathrm{H})\}_{2}\right]\left(\mathrm{I}_{\mathrm{Ar}}\right)$ underwent dehydrochlorination with $\mathrm{HSiCl}_{3}$ to form the NHCdichlorosilylene complex $\left[\mathrm{I}_{\mathrm{Ar}} \longrightarrow \mathrm{SiCl}_{2}\right] .{ }^{\mathrm{S} 12}$ Subsequently, the dehydrogenation of Int03 with $\left[\mathrm{I}_{\mathrm{Me}}-\mathrm{H}\right] \mathrm{I}$ via TS03 forms compound 3. ${ }^{\mathrm{S} 13}$ The Si lone pair electrons in 3 interact with $\mathrm{CO}_{2}$ to form Int04. Subsequently, the H-B bond of HBpin inserts into the C-O bond via a low kinetic barrier (TS04), which results in the formation of the formoxyborane $\mathbf{2 a}$ and regeneration of $\mathbf{3}$.

Scheme S1. Calculated Gibbs free energy profile (kcal/mol) in gas phase at $24{ }^{\circ} \mathrm{C}$. The abbreviations $\mathrm{Cpd}_{2} \mathrm{CO}_{2}, \mathrm{HBpin}, \mathrm{Int}, \mathrm{I}_{\mathrm{Me}}$ and $\mathrm{TS}$ stand for compound, carbon dioxide, pinacolborane, intermediate, $\left[: \mathrm{C}\{\mathrm{N}(\mathrm{Me}) \mathrm{C}(\mathrm{Me})\}_{2}\right]$ and transition state, respectively. Geometrical coordinates of the reactants, intermediates, transition states and compounds $\mathbf{1}$ - $\mathbf{3}$ are at M06-2X/def2-TZVP level of theory. No transition state was found between compound $\mathbf{1}$ and Int02, which indicates that the reaction from Cpd1 to Int02 should have a pretty low barrier, which is not easy to find out, or a non-barrier process. 


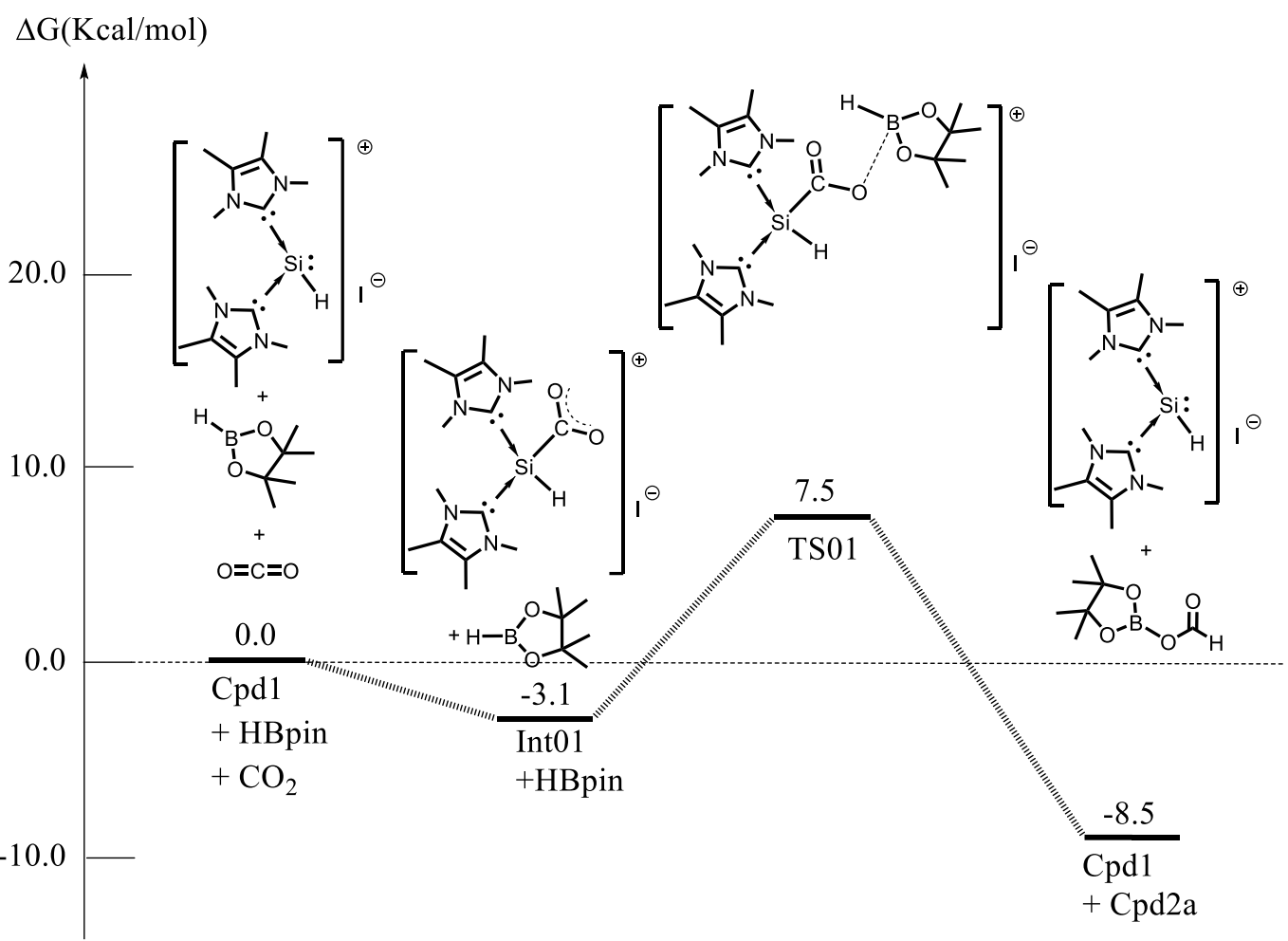

Scheme S2. Calculated Gibbs free energy profile (kcal/mol) in gas phase at $24{ }^{\circ} \mathrm{C}$. The abbreviations $\mathrm{Cpd}, \mathrm{CO}_{2}, \mathrm{HBpin}$, Int and $\mathrm{TS}$ stand for compound, carbon dioxide, pinacolborane, intermediate and transition state, respectively. Geometrical coordinates of the reactants, intermediates, transition states and compounds $\mathbf{1}$ and $\mathbf{2 a}$ are at M06-2X/def2-TZVP level of theory. 

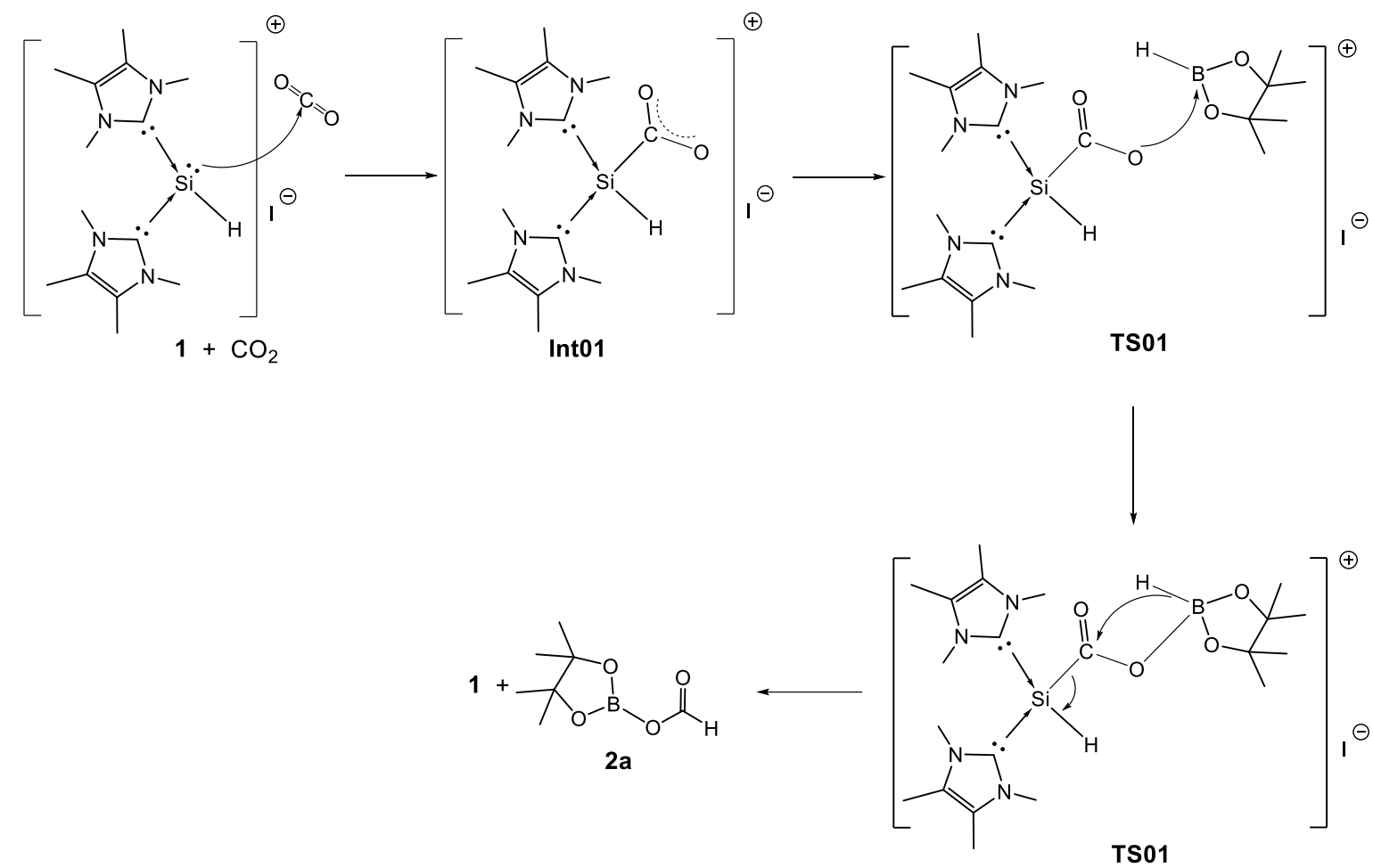

Scheme S3. Mechanism for the reaction of compound 1 with $\mathrm{CO}_{2}$ and $\mathrm{HBpin}$ to afford compound 2a with the regeneration of compound $\mathbf{1}$. 


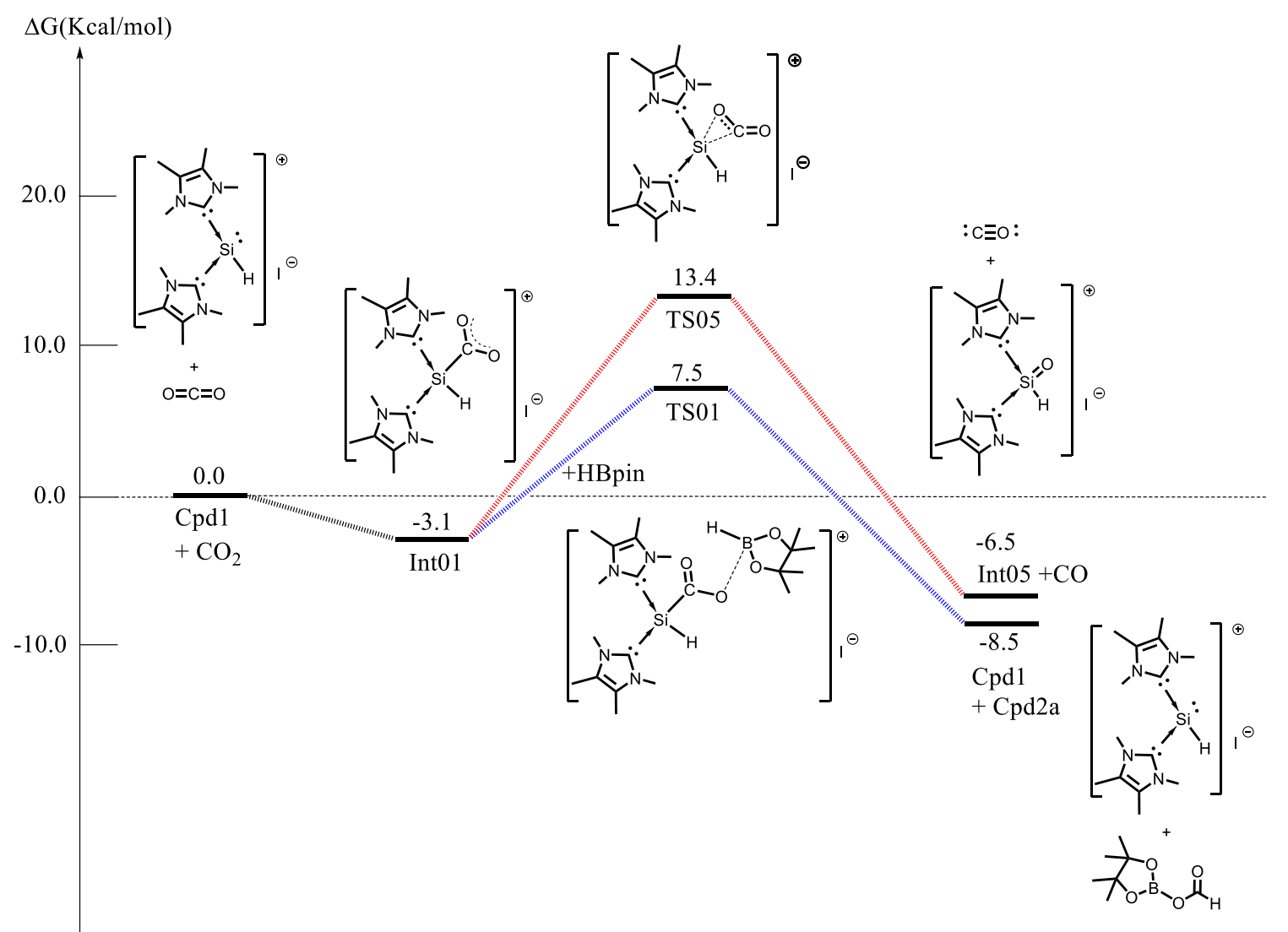

Scheme S4. Calculated Gibbs free energy profile ( $\mathrm{kcal} / \mathrm{mol})$ in gas phase at $24{ }^{\circ} \mathrm{C}$. The abbreviations $\mathrm{Cpd}, \mathrm{CO}_{2}$, Int and TS stand for compound, carbon dioxide, intermediate and transition state, respectively. Geometrical coordinates of the reactants, intermediates, transition states, compounds $\mathbf{1}$ and $\mathbf{2 a}$ are at M06-2X/def2-TZVP level of theory. 


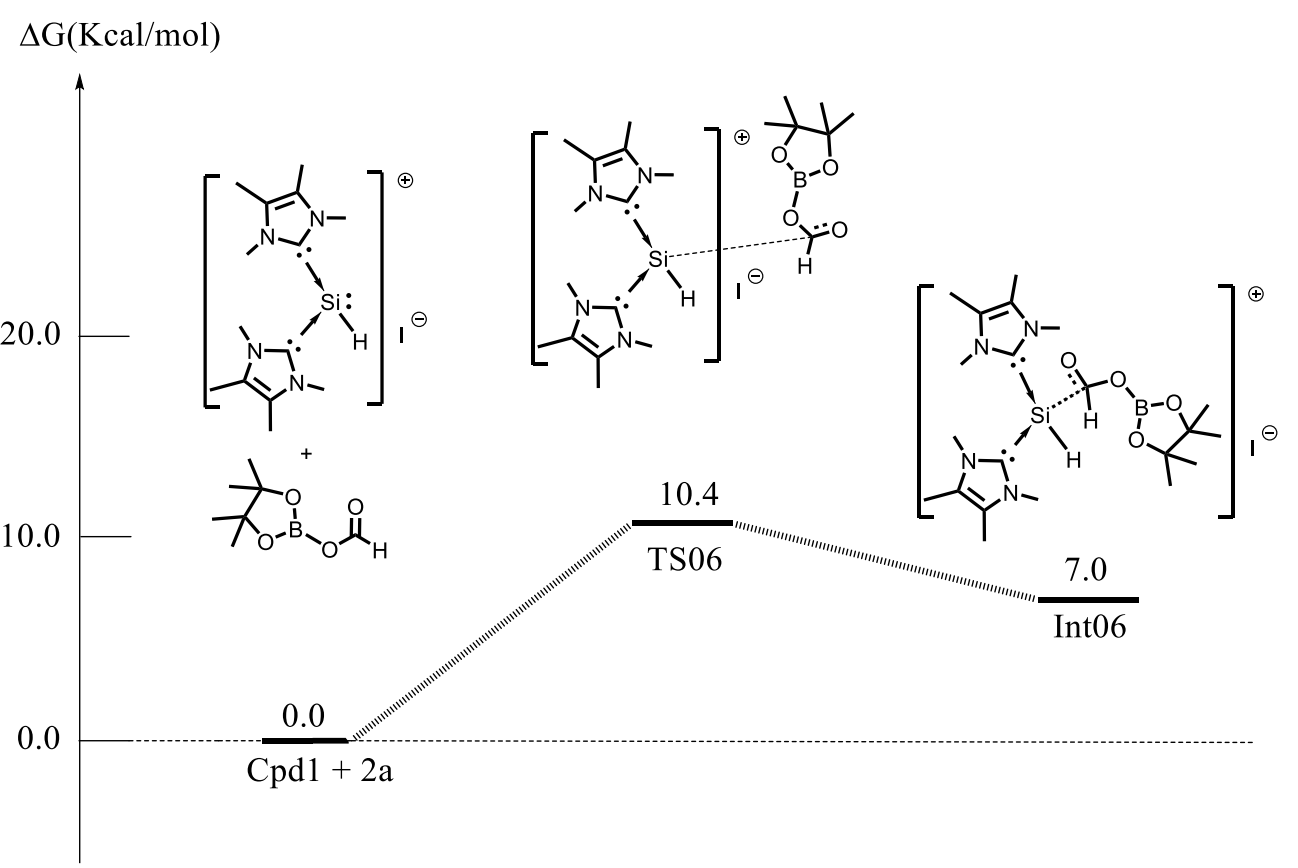

Scheme S5. Calculated Gibbs free energy profile ( $\mathrm{kcal} / \mathrm{mol}$ ) in gas phase at $24{ }^{\circ} \mathrm{C}$. The abbreviations Cpd, Int and TS stand for compound, intermediate and transition state, respectively. Geometrical coordinates of the reactants, intermediates, transition states and compounds $\mathbf{1}$ and $\mathbf{2 a}$ are at M06-2X/def2-TZVP level of theory. 
Table S14

M06-2X/def2-TZVP

Compound 1

$1^{\text {st }}$ frequency: $19.5 \mathrm{~cm}^{-1}$

Sum of electronic and thermal Free Energies $=-1354.293565$ Hartree

\begin{tabular}{|c|c|c|c|}
\hline \multirow{2}{*}{$\begin{array}{l}\text { Atomic } \\
\text { Number }\end{array}$} & \multicolumn{3}{|c|}{ Coordinates (Angstroms) } \\
\hline & $\mathrm{x}$ & Y & z \\
\hline 14 & -0.590631 & -0.964751 & 1.167401 \\
\hline 6 & -2.318192 & -0.426648 & 0.444038 \\
\hline 6 & -4.465731 & -0.595928 & -0.232511 \\
\hline 6 & -4.233514 & 0.706303 & 0.062913 \\
\hline 6 & -3.064275 & -2.699511 & -0.144590 \\
\hline 1 & -4.014680 & -3.181114 & -0.355313 \\
\hline 1 & -2.648374 & -3.108304 & 0.775964 \\
\hline 1 & -2.362554 & -2.892415 & -0.955351 \\
\hline 6 & -5.693837 & -1.274501 & -0.723529 \\
\hline 1 & -6.483336 & -0.542630 & -0.878327 \\
\hline 1 & -6.059248 & -2.012683 & -0.006950 \\
\hline 1 & -5.517339 & -1.783788 & -1.672684 \\
\hline 6 & -5.126723 & 1.892572 & -0.004700 \\
\hline 1 & -6.093471 & 1.605599 & -0.412166 \\
\hline 1 & -4.709203 & 2.670767 & -0.646389 \\
\hline 1 & -5.297369 & 2.325498 & 0.983102 \\
\hline 6 & -2.243251 & 1.987211 & 0.939911 \\
\hline 1 & -1.649035 & 1.744430 & 1.821367 \\
\hline 1 & -2.989397 & 2.731569 & 1.205534 \\
\hline 1 & -1.584288 & 2.387597 & 0.169012 \\
\hline 6 & 0.401503 & 0.449901 & 0.275994 \\
\hline 6 & 1.513572 & 1.652204 & -1.253381 \\
\hline 6 & 1.906240 & 2.080365 & -0.027028 \\
\hline 6 & 0.086551 & -0.218944 & -2.078160 \\
\hline 1 & -0.930368 & -0.530870 & -1.855662 \\
\hline 1 & 0.747754 & -1.089705 & -2.100034 \\
\hline 1 & 0.107762 & 0.298036 & -3.034096 \\
\hline 6 & 2.031539 & 1.989120 & -2.603251 \\
\hline 1 & 2.776964 & 2.777333 & -2.526269 \\
\hline 1 & 1.243541 & 2.332695 & -3.275995 \\
\hline 1 & 2.512565 & 1.112743 & -3.043544 \\
\hline 6 & 2.979694 & 3.023216 & 0.375083 \\
\hline 1 & 3.407513 & 3.498113 & -0.504631 \\
\hline 1 & 3.776795 & 2.477669 & 0.885714 \\
\hline 1 & 2.611927 & 3.806950 & 1.039706 \\
\hline 6 & 1.517918 & 1.331916 & 2.307057 \\
\hline 1 & 0.731332 & 0.820284 & 2.854005 \\
\hline 1 & 1.624379 & 2.351954 & 2.671799 \\
\hline 1 & 2.452274 & 0.778105 & 2.428317 \\
\hline 7 & -3.276674 & -1.270947 & 0.010281 \\
\hline 7 & -2.910835 & 0.784197 & 0.480003 \\
\hline 7 & 0.558560 & 0.677829 & -1.040433 \\
\hline 7 & 1.184207 & 1.350222 & 0.893972 \\
\hline 53 & 3.176670 & -1.382027 & 0.066991 \\
\hline 1 & -0.384575 & -1.996627 & 0.104920 \\
\hline
\end{tabular}




\section{Table S15}

\section{M06-2X/def2-TZVP}

HBpin

$1^{\text {st }}$ frequency: $108.6 \mathrm{~cm}^{-1}$

Sum of electronic and thermal Free Energies $=-411.690905$ Hartree

\begin{tabular}{|c|c|c|c|}
\hline Atomic & \multicolumn{3}{|c|}{ Coordinates (Angstroms) } \\
\hline Number & $\mathrm{x}$ & Y & Z \\
\hline 6 & 0.779184 & -0.185850 & 0,054683 \\
\hline 6 & -0.779184 & -0.185850 & -0.054683 \\
\hline 8 & -1.060939 & 1.185654 & -0.414203 \\
\hline 8 & 1.060939 & 1.185654 & 0.414203 \\
\hline 6 & -1.465948 & -0.440836 & 1.280943 \\
\hline 1 & -2.529825 & -0.231298 & 1.172188 \\
\hline 1 & -1.343785 & -1.476852 & 1.598567 \\
\hline 1 & -1.065051 & 0.213726 & 2.056004 \\
\hline 6 & -1.341506 & -1.104175 & -1.121494 \\
\hline 1 & -1.066036 & -2.140444 & -0.914452 \\
\hline 1 & -2.429198 & -1.033310 & -1.124086 \\
\hline 1 & -0.978871 & -0.833921 & -2.111084 \\
\hline 6 & 1.341505 & -1.104175 & 1.121494 \\
\hline 1 & 1.066035 & -2.140445 & 0.914452 \\
\hline 1 & 2.429198 & -1.033311 & 1.124085 \\
\hline 1 & 0.978872 & -0.833921 & 2.111083 \\
\hline 6 & 1.465949 & -0.440836 & -1.280943 \\
\hline 1 & 2.529825 & -0.231297 & -1.172188 \\
\hline 1 & 1.343786 & -1.476851 & -1.598566 \\
\hline 1 & 1.065050 & 0.213726 & -2.056005 \\
\hline 5 & 0.000000 & 1.936754 & 0.000000 \\
\hline 1 & 0.000000 & 3.120304 & 0.000000 \\
\hline
\end{tabular}


Table S16

M06-2X/def2-TZVP

Int01

$1^{\text {st }}$ frequency: $25.1 \mathrm{~cm}^{-1}$

Sum of electronic and thermal Free Energies $=-1542.904121$ Hartree

\begin{tabular}{|c|c|c|c|}
\hline Atomic & \multicolumn{3}{|c|}{ Coordinates (Angstroms) } \\
\hline Number & $\mathrm{X}$ & Y & $\mathrm{Z}$ \\
\hline--------- & ---------- & ---------- & ---------- \\
\hline 14 & -0.331917 & 2.129040 & -0.860746 \\
\hline 6 & 1.337716 & 1.192521 & -0.649807 \\
\hline 6 & 2.997037 & -0.280587 & -0.914938 \\
\hline 6 & 3.233599 & 0.410402 & 0.232700 \\
\hline 6 & 1.163035 & -0.349455 & -2.589233 \\
\hline 1 & 0.629651 & -1.239947 & -2.245924 \\
\hline 1 & 1.897108 & -0.616970 & -3.345585 \\
\hline 1 & 0.473258 & 0.376083 & -3.010779 \\
\hline 6 & 3.695101 & -1.444280 & -1.513699 \\
\hline 1 & 4.560258 & -1.708145 & -0.910449 \\
\hline 1 & 4.037634 & -1.237185 & -2.529010 \\
\hline 1 & 3.018380 & -2.302228 & -1.527017 \\
\hline 6 & 4.291954 & 0.255095 & 1.261158 \\
\hline 1 & 5.002321 & -0.506161 & 0.948781 \\
\hline 1 & 3.856358 & -0.061806 & 2.210682 \\
\hline 1 & 4.838993 & 1.184688 & 1.425484 \\
\hline 6 & 2.104028 & 2.253589 & 1.476919 \\
\hline 1 & 3.044929 & 2.792746 & 1.572372 \\
\hline 1 & 1.916590 & 1.699122 & 2.396472 \\
\hline 1 & 1.303988 & 2.974546 & 1.301385 \\
\hline 6 & -1.576333 & 0.778109 & -0.356148 \\
\hline 6 & -2.716826 & -0.613447 & 0.964029 \\
\hline 6 & -3.066237 & -0.885369 & -0.320545 \\
\hline 6 & -1.085427 & 0.905648 & 2.073481 \\
\hline 1 & -0.769040 & 1.934621 & 1.916739 \\
\hline 1 & -0.231515 & 0.240684 & 2.228172 \\
\hline 1 & -1.739438 & 0.878851 & 2.940793 \\
\hline 6 & -3.069458 & -1.297815 & 2.231540 \\
\hline 1 & -3.764659 & -2.109755 & 2.032992 \\
\hline 1 & -3.534701 & -0.617042 & 2.946059 \\
\hline 1 & -2.166996 & -1.726311 & 2.673781 \\
\hline 6 & -3.939179 & -1.941143 & -0.890614 \\
\hline 1 & -4.382813 & -2.524494 & -0.087865 \\
\hline 1 & -3.356435 & -2.621885 & -1.514539 \\
\hline 1 & -4.748461 & -1.522733 & -1.491544 \\
\hline 6 & -2.444592 & -0.004547 & -2.560385 \\
\hline 1 & -1.969190 & 0.889819 & -2.948593 \\
\hline 1 & -3.490576 & -0.000893 & -2.859741 \\
\hline 1 & -1.956535 & -0.893993 & -2.959874 \\
\hline 7 & 1.841660 & 0.238684 & -1.449597 \\
\hline 7 & 2.206036 & 1.317565 & 0.364652 \\
\hline 7 & -1.820051 & 0.427722 & 0.913277 \\
\hline 7 & -2.357628 & -0.007361 & -1.112724 \\
\hline 53 & 0.401032 & -2.272279 & 0.529934 \\
\hline 1 & -0.439665 & 2.308288 & -2.316866 \\
\hline 6 & -0.871216 & 3.709139 & 0.120310 \\
\hline 8 & -0.281370 & 4.287296 & 1.022334 \\
\hline 8 & -1.974463 & 3.846567 & -0.432313 \\
\hline
\end{tabular}


Table S17

M06-2X/def2-TZVP

Int02

$1^{\text {st }}$ frequency: $5.5 \mathrm{~cm}^{-1}$

Sum of electronic and thermal Free Energies $=-1382.717342$ Hartree

\begin{tabular}{|c|c|c|c|}
\hline \multirow{2}{*}{$\begin{array}{l}\text { Atomic } \\
\text { Number }\end{array}$} & \multicolumn{3}{|c|}{ Coordinates (Angstroms) } \\
\hline & $\mathrm{x}$ & Y & z \\
\hline 14 & 0.327005 & -1.218172 & 0.186856 \\
\hline 6 & 4.528114 & -0.670858 & 0.347273 \\
\hline 6 & 4.081894 & 0.625248 & -0.397423 \\
\hline 6 & -0.690687 & 0.383477 & 0.223176 \\
\hline 6 & -2.129304 & 1.963823 & 0.839946 \\
\hline 6 & -1.924091 & 2.088610 & -0.498402 \\
\hline 6 & -1.414616 & 0.304408 & 2.575209 \\
\hline 1 & -0.435962 & -0.055141 & 2.882950 \\
\hline 1 & -2.119433 & -0.528390 & 2.500617 \\
\hline 1 & -1.765656 & 1.035541 & 3.297695 \\
\hline 6 & -3.066144 & 2.673147 & 1.747140 \\
\hline 1 & -3.595341 & 3.449444 & 1.199721 \\
\hline 1 & -2.547284 & 3.144154 & 2.583921 \\
\hline 1 & -3.806601 & 1.974686 & 2.142124 \\
\hline 6 & -2.561665 & 2.981098 & -1.498895 \\
\hline 1 & -3.236838 & 3.672736 & -1.000832 \\
\hline 1 & -3.145270 & 2.394442 & -2.211210 \\
\hline 1 & -1.824128 & 3.567246 & -2.049922 \\
\hline 6 & -0.656918 & 0.746508 & -2.203331 \\
\hline 1 & 0.383959 & 0.428953 & -2.237736 \\
\hline 1 & -0.785013 & 1.603187 & -2.858872 \\
\hline 1 & -1.321272 & -0.067354 & -2.506517 \\
\hline 7 & -1.320112 & 0.932265 & 1.269068 \\
\hline 7 & -0.998534 & 1.127116 & -0.844567 \\
\hline 53 & -3.339345 & -1.441768 & -0.259618 \\
\hline 1 & -0.013828 & -1.987053 & 1.391323 \\
\hline 1 & -0.009933 & -1.923016 & -1.058405 \\
\hline 5 & 2.268802 & -0.643393 & 0.130194 \\
\hline 8 & 2.706025 & 0.318176 & -0.742640 \\
\hline 8 & 3.275628 & -1.155158 & 0.895022 \\
\hline 6 & 5.037909 & -1.751058 & -0.597554 \\
\hline 1 & 6.010714 & -1.486542 & -1.013224 \\
\hline 1 & 5.136644 & -2.683995 & -0.043501 \\
\hline 1 & 4.337265 & -1.912645 & -1.417967 \\
\hline 6 & 5.509326 & -0.449207 & 1.480367 \\
\hline 1 & 5.751436 & -1.406311 & 1.941958 \\
\hline 1 & 6.433411 & -0.008454 & 1.100582 \\
\hline 1 & 5.095710 & 0.204744 & 2.244951 \\
\hline 6 & 4.855252 & 0.928486 & -1.664602 \\
\hline 1 & 4.479925 & 1.851206 & -2.107217 \\
\hline 1 & 5.914729 & 1.064784 & -1.438209 \\
\hline 1 & 4.752358 & 0.129616 & -2.395800 \\
\hline 6 & 4.042930 & 1.848219 & 0.509176 \\
\hline 1 & 5.049000 & 2.177907 & 0.770397 \\
\hline 1 & 3.535759 & 2.657985 & -0.014923 \\
\hline 1 & 3.494144 & 1.636109 & 1.427843 \\
\hline
\end{tabular}




\section{Table S18}

\section{M06-2X/def2-TZVP}

$\mathrm{I}_{\mathrm{Me}}$

$1^{\text {st }}$ frequency: $108.5 \mathrm{~cm}^{-1}$

Sum of electronic and thermal Free Energies $=-383.254033$ Hartree

\begin{tabular}{|c|c|c|c|}
\hline \multirow{2}{*}{$\begin{array}{l}\text { Atomic } \\
\text { Number }\end{array}$} & \multicolumn{3}{|c|}{ Coordinates (Angstroms) } \\
\hline & $\mathrm{x}$ & Y & z \\
\hline 6 & -0.000000 & -1.563410 & 0.000022 \\
\hline 6 & 0.676943 & 0.630607 & -0.000038 \\
\hline 6 & -0.676942 & 0.630607 & 0.000006 \\
\hline 6 & 2.428343 & -1.152289 & 0.000013 \\
\hline 1 & 2.954135 & -0.794835 & -0.887318 \\
\hline 1 & 2.417490 & -2.238629 & -0.000224 \\
\hline 1 & 2.953957 & -0.795218 & 0.887603 \\
\hline 6 & 1.661043 & 1.745433 & -0.000005 \\
\hline 1 & 1.145482 & 2.704497 & -0.000293 \\
\hline 1 & 2.305969 & 1.711605 & -0.881252 \\
\hline 1 & 2.305578 & 1.711906 & 0.881538 \\
\hline 6 & -1.661042 & 1.745433 & 0.000016 \\
\hline 1 & -1.145481 & 2.704498 & -0.000026 \\
\hline 1 & -2.305738 & 1.711780 & 0.881439 \\
\hline 1 & -2.305809 & 1.711731 & -0.881352 \\
\hline 6 & -2.428343 & -1.152289 & -0.000033 \\
\hline 1 & -2.417490 & -2.238628 & 0.000404 \\
\hline 1 & -2.953881 & -0.795383 & -0.887735 \\
\hline 1 & -2.954211 & -0.794670 & 0.887187 \\
\hline 7 & 1.053525 & -0.707371 & -0.000032 \\
\hline 7 & -1.053525 & -0.707371 & 0.000053 \\
\hline
\end{tabular}


Table S19

M06-2X/def2-TZVP

Int03

$1^{\text {st }}$ frequency: $13.9 \mathrm{~cm}^{-1}$

Sum of electronic and thermal Free Energies $=-1084.428423$ Hartree

\begin{tabular}{|c|c|c|c|}
\hline \multirow{2}{*}{$\begin{array}{l}\text { Atomic } \\
\text { Number }\end{array}$} & \multicolumn{3}{|c|}{ Coordinates (Angstroms) } \\
\hline & $\mathrm{x}$ & Y & z \\
\hline 14 & 0.289225 & 1.593533 & -1.389159 \\
\hline 6 & -3.455083 & 0.365277 & 0.097274 \\
\hline 6 & -2.593485 & -0.828533 & 0.600612 \\
\hline 6 & 1.689943 & 0.600264 & -0.534787 \\
\hline 6 & 3.609973 & 0.037665 & 0.520099 \\
\hline 6 & 3.129387 & -1.079217 & -0.075390 \\
\hline 6 & 2.837750 & 2.435507 & 0.655036 \\
\hline 1 & 1.974212 & 2.716225 & 1.256184 \\
\hline 1 & 2.881869 & 3.094692 & -0.211152 \\
\hline 1 & 3.747183 & 2.544079 & 1.239582 \\
\hline 6 & 4.827752 & 0.242165 & 1.347834 \\
\hline 1 & 5.360318 & -0.700229 & 1.456609 \\
\hline 1 & 4.579325 & 0.603119 & 2.347932 \\
\hline 1 & 5.511307 & 0.960739 & 0.890952 \\
\hline 6 & 3.650018 & -2.471031 & -0.104902 \\
\hline 1 & 4.523117 & -2.556900 & 0.538477 \\
\hline 1 & 3.945849 & -2.770281 & -1.112593 \\
\hline 1 & 2.898904 & -3.180879 & 0.247127 \\
\hline 6 & 1.148292 & -1.601613 & -1.529601 \\
\hline 1 & 0.648204 & -1.009180 & -2.296621 \\
\hline 1 & 0.389475 & -2.090609 & -0.922658 \\
\hline 1 & 1.792481 & -2.338644 & -2.005283 \\
\hline 7 & 2.713128 & 1.057128 & 0.224083 \\
\hline 7 & 1.959960 & -0.710623 & -0.723323 \\
\hline 1 & 0.392511 & 2.815340 & -0.521783 \\
\hline 5 & -1.266307 & 0.699159 & -0.475080 \\
\hline 8 & -1.367643 & -0.644110 & -0.132836 \\
\hline 8 & -2.453345 & 1.351610 & -0.204520 \\
\hline 6 & -4.187111 & 0.055220 & -1.203930 \\
\hline 1 & -5.006534 & -0.647922 & -1.046621 \\
\hline 1 & -4.594140 & 0.984089 & -1.602933 \\
\hline 1 & -3.500556 & -0.360371 & -1.943028 \\
\hline 6 & -4.420531 & 0.929292 & 1.122195 \\
\hline 1 & -4.980188 & 1.752439 & 0.677612 \\
\hline 1 & -5.130949 & 0.163170 & 1.441557 \\
\hline 1 & -3.893291 & 1.307885 & 1.995659 \\
\hline 6 & -3.160303 & -2.200269 & 0.288499 \\
\hline 1 & -2.495501 & -2.969091 & 0.684575 \\
\hline 1 & -4.140607 & -2.324954 & 0.754051 \\
\hline 1 & -3.260609 & -2.352338 & -0.784575 \\
\hline 6 & -2.250260 & -0.722270 & 2.082337 \\
\hline 1 & -3.122351 & -0.911655 & 2.710186 \\
\hline 1 & -1.484260 & -1.461847 & 2.317287 \\
\hline 1 & -1.855159 & 0.267576 & 2.314948 \\
\hline
\end{tabular}


Table S20

M06-2X/def2-TZVP

$\left[\mathrm{I}_{\mathrm{Me}}-\mathrm{H}\right] \mathrm{I}$

$1^{\text {st }}$ frequency: $35.4 \mathrm{~cm}^{-1}$

Sum of electronic and thermal Free Energies $=-681.539006$ Hartree

\begin{tabular}{|c|c|c|c|}
\hline Atomic & \multicolumn{3}{|c|}{ Coordinates (Angstroms) } \\
\hline Number & $\mathrm{x}$ & Y & Z \\
\hline 6 & 0.802451 & -0.000017 & -1.509565 \\
\hline 6 & 1.835130 & -0.679597 & 0.309473 \\
\hline 6 & 1.835122 & 0.679640 & 0.309451 \\
\hline 6 & 0.737796 & -2.419523 & -1.140113 \\
\hline 1 & 0.483288 & -2.491257 & -2.194520 \\
\hline 1 & -0.159339 & -2.586733 & -0.539419 \\
\hline 1 & 1.513820 & -3.143725 & -0.904387 \\
\hline 6 & 2.276061 & -1.658918 & 1.332668 \\
\hline 1 & 2.744900 & -1.141274 & 2.165984 \\
\hline 1 & 2.995244 & -2.373434 & 0.928818 \\
\hline 1 & 1.413970 & -2.208571 & 1.716869 \\
\hline 6 & 2.276012 & 1.659004 & 1.332625 \\
\hline 1 & 2.744872 & 1.141402 & 2.165955 \\
\hline 1 & 1.413897 & 2.208631 & 1.716809 \\
\hline 1 & 2.995162 & 2.373545 & 0.928759 \\
\hline 6 & 0.737711 & 2.419491 & -1.140141 \\
\hline 1 & 0.482870 & 2.491101 & -2.194476 \\
\hline 1 & 1.513801 & 3.143733 & -0.904753 \\
\hline 1 & -0.159240 & 2.586724 & -0.539181 \\
\hline 7 & 1.220081 & -1.078611 & -0.864322 \\
\hline 7 & 1.220066 & 1.078605 & -0.864356 \\
\hline 53 & -1.854399 & -0.000010 & 0.286782 \\
\hline 1 & 0.217155 & -0.000034 & -2.411559 \\
\hline
\end{tabular}


Table S21

M06-2X/def2-TZVP

Int04

$1^{\text {st }}$ frequency: $12.6 \mathrm{~cm}^{-1}$

Sum of electronic and thermal Free Energies $=-1953.401962$ Hartree

\begin{tabular}{|c|c|c|c|}
\hline Atomic & \multicolumn{3}{|c|}{ Coordinates (Angstroms) } \\
\hline Number & $\mathrm{X}$ & Y & $\mathrm{Z}$ \\
\hline------ & ---------- & ---------- & ----------1 \\
\hline 14 & 0.153531 & 0.104545 & -1.081748 \\
\hline 6 & 1.578513 & -1.163223 & -0.912439 \\
\hline 6 & 2.834287 & -2.957318 & -0.476520 \\
\hline 6 & 3.655480 & -1.993161 & -0.973611 \\
\hline 6 & 0.404243 & -3.189264 & -0.009904 \\
\hline 1 & 0.428495 & -3.273627 & 1.075381 \\
\hline 1 & 0.438700 & -4.168894 & -0.484717 \\
\hline 1 & -0.510200 & -2.681869 & -0.294837 \\
\hline 6 & 3.119254 & -4.340992 & -0.016762 \\
\hline 1 & 4.193061 & -4.510920 & 0.010830 \\
\hline 1 & 2.676280 & -5.081719 & -0.685192 \\
\hline 1 & 2.718833 & -4.507580 & 0.984024 \\
\hline 6 & 5.119026 & -1.996697 & -1.230679 \\
\hline 1 & 5.558477 & -2.918017 & -0.855297 \\
\hline 1 & 5.610607 & -1.158625 & -0.733992 \\
\hline 1 & 5.336321 & -1.928922 & -2.298274 \\
\hline 6 & 3.364119 & 0.345759 & -1.805891 \\
\hline 1 & 4.097745 & 0.109934 & -2.574081 \\
\hline 1 & 3.845750 & 0.929171 & -1.020183 \\
\hline 1 & 2.551022 & 0.904017 & -2.278413 \\
\hline 6 & 0.749754 & 1.676040 & -0.139916 \\
\hline 6 & 1.718342 & 3.268601 & 1.097748 \\
\hline 6 & 0.758848 & 3.862736 & 0.342537 \\
\hline 6 & 2.580703 & 0.980529 & 1.446452 \\
\hline 1 & 2.377972 & -0.024068 & 1.099657 \\
\hline 1 & 2.369154 & 0.988928 & 2.513873 \\
\hline 1 & 3.618132 & 1.262454 & 1.258804 \\
\hline 6 & 2.638749 & 3.825962 & 2.121955 \\
\hline 1 & 2.485898 & 4.898578 & 2.214777 \\
\hline 1 & 3.684203 & 3.654593 & 1.859154 \\
\hline 1 & 2.458201 & 3.372299 & 3.098140 \\
\hline 6 & 0.329213 & 5.283213 & 0.265324 \\
\hline 1 & 0.971934 & 5.898081 & 0.891153 \\
\hline 1 & -0.697711 & 5.406670 & 0.613817 \\
\hline 1 & 0.388764 & 5.659958 & -0.756710 \\
\hline 6 & -0.861695 & 3.110966 & -1.406572 \\
\hline 1 & -0.402294 & 3.255285 & -2.384000 \\
\hline 1 & -1.420066 & 3.992418 & -1.103864 \\
\hline 1 & -1.547859 & 2.269558 & -1.442561 \\
\hline 7 & 1.562866 & -2.426248 & -0.462356 \\
\hline 7 & 2.856863 & -0.905427 & -1.252606 \\
\hline 7 & 1.701233 & 1.926081 & 0.777084 \\
\hline 7 & 0.179113 & 2.863743 & -0.409626 \\
\hline 53 & -0.240354 & -0.713861 & 2.533191 \\
\hline 6 & -3.703083 & -1.431227 & -0.268295 \\
\hline 6 & -3.912418 & 0.105254 & -0.451890 \\
\hline 8 & -2.311165 & -1.593867 & -0.650226 \\
\hline 8 & -2.580052 & 0.628487 & -0.218098 \\
\hline 5 & -1.702168 & -0.384278 & -0.492963 \\
\hline 6 & -4.552993 & -2.292185 & -1.181711 \\
\hline 1 & -4.346329 & -3.344235 & -0.984217 \\
\hline 1 & -5.612934 & -2.111166 & -0.990608 \\
\hline 1 & -4.341411 & -2.087753 & -2.229438 \\
\hline 6 & -3.828311 & -1.884440 & 1.178020 \\
\hline 1 & -4.866172 & -1.836923 & 1.511379 \\
\hline
\end{tabular}




$\begin{array}{rrr}-3.486123 & -2.917309 & 1.252266 \\ -3.203373 & -1.280088 & 1.836179 \\ -4.276146 & 0.490772 & -1.880079 \\ -5.279744 & 0.151833 & -2.140038 \\ -4.249103 & 1.578112 & -1.964519 \\ -3.554567 & 0.082265 & -2.590475 \\ -4.866057 & 0.731659 & 0.544680 \\ -4.953397 & 1.799443 & 0.342241 \\ -5.857922 & 0.284390 & 0.449854 \\ -4.512060 & 0.600120 & 1.565078 \\ 0.053131 & 0.688988 & -2.947951 \\ -1.012992 & 0.332303 & -3.461915 \\ 0.980909 & 1.393963 & -3.388443\end{array}$

Page S113 
Table S22

M06-2X/def2-TZVP

Compound 3

$1^{\text {st }}$ frequency: $17.4 \mathrm{~cm}^{-1}$

Sum of electronic and thermal Free Energies $=-1764.803014$ Hartree

\begin{tabular}{|c|c|c|c|}
\hline \multirow{2}{*}{$\begin{array}{l}\text { Atomic } \\
\text { Number }\end{array}$} & \multicolumn{3}{|c|}{ Coordinates (Angstroms) } \\
\hline & $\mathrm{x}$ & Y & z \\
\hline 14 & 1.050232 & -0.033915 & 1.682659 \\
\hline 6 & 2.650969 & -0.338326 & 0.634772 \\
\hline 6 & 4.683723 & -0.017382 & -0.294074 \\
\hline 6 & 4.422625 & -1.344447 & -0.343043 \\
\hline 6 & 3.522226 & 1.992686 & 0.646983 \\
\hline 1 & 4.528487 & 2.354369 & 0.846208 \\
\hline 1 & 2.921672 & 2.119639 & 1.546193 \\
\hline 1 & 3.064184 & 2.555168 & -0.163336 \\
\hline 6 & 5.856506 & 0.762766 & -0.767746 \\
\hline 1 & 6.523900 & 0.120579 & -1.338248 \\
\hline 1 & 6.425957 & 1.181205 & 0.064791 \\
\hline 1 & 5.548113 & 1.588049 & -1.411659 \\
\hline 6 & 5.221465 & -2.474139 & -0.886103 \\
\hline 1 & 6.089626 & -2.091499 & -1.418282 \\
\hline 1 & 4.635169 & -3.075396 & -1.583477 \\
\hline 1 & 5.580859 & -3.134490 & -0.094125 \\
\hline 6 & 2.596803 & -2.828681 & 0.510300 \\
\hline 1 & 2.006645 & -2.776365 & 1.423339 \\
\hline 1 & 3.400626 & -3.547451 & 0.650415 \\
\hline 1 & 1.956518 & -3.156328 & -0.308869 \\
\hline 6 & -0.049869 & -1.320062 & 0.724870 \\
\hline 6 & -0.903387 & -2.729226 & -0.800564 \\
\hline 6 & -1.418558 & -3.070975 & 0.407698 \\
\hline 6 & 0.499380 & -0.883146 & -1.670295 \\
\hline 1 & 0.893602 & 0.052682 & -1.287577 \\
\hline 1 & -0.297799 & -0.656831 & -2.379582 \\
\hline 1 & 1.291898 & -1.448351 & -2.166774 \\
\hline 6 & -1.261792 & -3.194594 & -2.162657 \\
\hline 1 & -1.758481 & -4.160977 & -2.110640 \\
\hline 1 & -0.387467 & -3.292431 & -2.808173 \\
\hline 1 & -1.958934 & -2.477365 & -2.606924 \\
\hline 6 & -2.506651 & -4.016460 & 0.756030 \\
\hline 1 & -2.779501 & -4.604274 & -0.117382 \\
\hline 1 & -3.387345 & -3.444072 & 1.059615 \\
\hline 1 & -2.224181 & -4.699557 & 1.558693 \\
\hline 6 & -1.373425 & -2.077607 & 2.681369 \\
\hline 1 & -0.667935 & -1.495476 & 3.269569 \\
\hline 1 & -1.495738 & -3.065668 & 3.119916 \\
\hline 1 & -2.335085 & -1.561455 & 2.635914 \\
\hline 7 & 3.591583 & 0.577100 & 0.320769 \\
\hline 7 & 3.177855 & -1.523126 & 0.246391 \\
\hline 7 & -0.073668 & -1.650279 & -0.581402 \\
\hline 7 & -0.869895 & -2.203130 & 1.325581 \\
\hline 53 & -3.454466 & -0.384082 & -0.422194 \\
\hline 6 & -0.274829 & 3.213726 & -0.772910 \\
\hline 6 & -1.061082 & 3.458938 & 0.557030 \\
\hline 8 & 0.729622 & 2.245291 & -0.366424 \\
\hline
\end{tabular}




$\begin{array}{rrr}-0.807006 & 2.251180 & 1.307687 \\ 0.271829 & 1.618338 & 0.775015 \\ 0.431653 & 4.437248 & -1.327141 \\ 0.955795 & 4.167502 & -2.244835 \\ -0.295237 & 5.215277 & -1.569046 \\ 1.154467 & 4.844764 & -0.621639 \\ -1.112429 & 2.551380 & -1.855971 \\ -1.861983 & 3.239389 & -2.249521 \\ -0.452755 & 2.258217 & -2.675332 \\ -1.627534 & 1.664021 & -1.480262 \\ -0.488131 & 4.602208 & 1.386270 \\ -0.670616 & 5.568607 & 0.914452 \\ -0.967888 & 4.594455 & 2.364550 \\ 0.586912 & 4.479285 & 1.533849 \\ -2.558253 & 3.619205 & 0.380757 \\ -3.016051 & 3.802927 & 1.353094 \\ -2.775948 & 4.471132 & -0.268010 \\ -3.003348 & 2.715806 & -0.035851\end{array}$


Table S23

B3LYP/6-311 ${ }^{++}$G(2df,2pd)//B3LYP/6-31G**

Compound 3

\begin{tabular}{|c|c|c|c|}
\hline \multirow{2}{*}{$\begin{array}{l}\text { Atomic } \\
\text { Number }\end{array}$} & \multicolumn{3}{|c|}{ Coordinates (Angstroms) } \\
\hline & $\mathrm{X}$ & $\mathrm{Y}$ & Z \\
\hline---- & ----------- & ----------- & ---------- \\
\hline 6 & 0.029356 & 1.749042 & 0.851462 \\
\hline 6 & 1.044426 & 3.671426 & 0.191445 \\
\hline 6 & 1.977924 & 2.923109 & 0.847986 \\
\hline 6 & 3.425501 & 3.173942 & 1.106714 \\
\hline 1 & 3.641135 & 3.264595 & 2.177681 \\
\hline 1 & 4.039075 & 2.361872 & 0.700208 \\
\hline 1 & 3.735280 & 4.104055 & 0.626923 \\
\hline 6 & 1.151893 & 5.009722 & -0.459523 \\
\hline 1 & 2.166089 & 5.397021 & -0.349895 \\
\hline 1 & 0.930084 & 4.962035 & -1.531618 \\
\hline 1 & 0.466463 & 5.738178 & -0.011213 \\
\hline 6 & 1.987330 & 0.719644 & 2.048238 \\
\hline 1 & 1.231903 & 0.187016 & 2.626920 \\
\hline 1 & 2.536873 & 0.033329 & 1.397718 \\
\hline 1 & 2.689451 & 1.189175 & 2.737864 \\
\hline 6 & -1.373696 & 3.444287 & -0.390289 \\
\hline 1 & -1.207843 & 3.667109 & -1.447820 \\
\hline 1 & -2.170694 & 2.711624 & -0.305504 \\
\hline 1 & -1.678107 & 4.362810 & 0.119155 \\
\hline 7 & 1.330736 & 1.761243 & 1.260286 \\
\hline 7 & -0.141797 & 2.936269 & 0.204509 \\
\hline 6 & -0.331301 & -1.219207 & 0.826934 \\
\hline 6 & 0.431301 & -3.355385 & 0.883607 \\
\hline 6 & 0.795693 & -2.819021 & -0.320881 \\
\hline 6 & 1.571589 & -3.399018 & -1.454822 \\
\hline 1 & 0.987034 & -3.412924 & -2.381837 \\
\hline 1 & 2.485749 & -2.821079 & -1.633538 \\
\hline 1 & 1.859587 & -4.426773 & -1.226714 \\
\hline 6 & 0.666990 & -4.713146 & 1.456350 \\
\hline 1 & 1.189015 & -5.338774 & 0.730677 \\
\hline 1 & 1.283947 & -4.672765 & 2.361095 \\
\hline 1 & -0.272816 & -5.213421 & 1.715937 \\
\hline 6 & 0.475564 & -0.623072 & -1.480265 \\
\hline 1 & -0.151379 & 0.253342 & -1.333412 \\
\hline 1 & 1.525385 & -0.327777 & -1.572526 \\
\hline 1 & 0.161530 & -1.142174 & -2.388443 \\
\hline 6 & -0.824854 & -2.548537 & 2.900228 \\
\hline 1 & -1.685484 & -3.220565 & 2.847871 \\
\hline 1 & -0.073586 & -2.974115 & 3.568880 \\
\hline 1 & -1.148774 & -1.578062 & 3.278033 \\
\hline 7 & 0.309416 & -1.515859 & -0.334173 \\
\hline 7 & -0.250498 & -2.356442 & 1.570928 \\
\hline 14 & -1.244247 & 0.400469 & 1.435249 \\
\hline 1 & -2.434529 & 1.759814 & 4.732055 \\
\hline 53 & 4.576289 & -0.462369 & -0.964526 \\
\hline 6 & -4.924315 & 0.647290 & -0.710825 \\
\hline 6 & -4.537966 & -0.867647 & -0.896604 \\
\hline 6 & -4.051890 & -1.207084 & -2.311930 \\
\hline 1 & -3.615843 & -2.209823 & -2.305549 \\
\hline 1 & -4.873404 & -1.195495 & -3.034237 \\
\hline
\end{tabular}




$\begin{array}{lrr}-3.283628 & -0.505841 & -2.649627 \\ -5.604351 & -1.868033 & -0.455257 \\ -6.514706 & -1.754703 & -1.052983 \\ -5.232818 & -2.886847 & -0.597875 \\ -5.861823 & -1.746701 & 0.598293 \\ -5.888664 & 0.896165 & 0.455535 \\ -5.948229 & 1.971454 & 0.644702 \\ -6.895008 & 0.531844 & 0.229211 \\ -5.540268 & 0.410933 & 1.371325 \\ -5.424288 & 1.353287 & -1.968732 \\ -6.354656 & 0.899055 & -2.324834 \\ -5.628902 & 2.403988 & -1.743491 \\ -4.689239 & 1.316320 & -2.774635 \\ -2.840414 & 0.246821 & 0.191986 \\ -2.687972 & 2.082795 & 5.352961 \\ -3.643709 & 1.242889 & -0.325318 \\ -3.382931 & -1.005225 & -0.013732\end{array}$


Table S24

M06-2X/def2-TZVP

TS01

$1^{\text {st }}$ frequency: $-99.7 \mathrm{~cm}^{-1}$

Sum of electronic and thermal Free Energies $=-1954.578146$ Hartree

\begin{tabular}{|c|c|c|c|}
\hline \multirow{2}{*}{$\begin{array}{l}\text { Atomic } \\
\text { Number }\end{array}$} & \multicolumn{3}{|c|}{ Coordinates (Angstroms) } \\
\hline & $\mathrm{X}$ & Y & Z \\
\hline----- & ---ー------ & ----------- & - - - - - - - - - \\
\hline 14 & 0.602873 & -0.705015 & -0.524897 \\
\hline 6 & -0.287606 & 0.744150 & 0.310075 \\
\hline 6 & -1.494668 & 2.594590 & 0.605425 \\
\hline 6 & -1.753025 & 1.760012 & 1.650187 \\
\hline 6 & -0.106769 & 2.473200 & -1.476980 \\
\hline 1 & 0.856865 & 2.026431 & -1.715766 \\
\hline 1 & 0.037014 & 3.545967 & -1.377231 \\
\hline 1 & -0.841240 & 2.268611 & -2.255981 \\
\hline 6 & -2.055127 & 3.927411 & 0.267259 \\
\hline 1 & -2.793155 & 4.217349 & 1.011767 \\
\hline 1 & -2.548138 & 3.906739 & -0.706699 \\
\hline 1 & -1.280648 & 4.695538 & 0.240745 \\
\hline 6 & -2.691107 & 1.875213 & 2.794122 \\
\hline 1 & -3.238442 & 2.813681 & 2.737979 \\
\hline 1 & -2.167972 & 1.847217 & 3.751723 \\
\hline 1 & -3.405539 & 1.049646 & 2.760191 \\
\hline 6 & -1.035591 & -0.526612 & 2.323980 \\
\hline 1 & -2.010904 & -1.001062 & 2.235750 \\
\hline 1 & -0.864051 & -0.203728 & 3.350381 \\
\hline 1 & -0.269022 & -1.243963 & 2.039309 \\
\hline 6 & 2.254657 & -1.072989 & 0.337654 \\
\hline 6 & 4.157159 & -1.062948 & 1.492118 \\
\hline 6 & 4.272900 & -1.998317 & 0.510742 \\
\hline 6 & 2.428004 & 0.631643 & 2.124472 \\
\hline 1 & 1.419900 & 0.465015 & 2.493620 \\
\hline 1 & 2.466732 & 1.497692 & 1.459131 \\
\hline 1 & 3.086018 & 0.791047 & 2.972858 \\
\hline 6 & 5.151142 & -0.583394 & 2.485825 \\
\hline 1 & 6.070548 & -1.155808 & 2.391271 \\
\hline 1 & 4.790659 & -0.695018 & 3.509678 \\
\hline 1 & 5.388317 & 0.467246 & 2.307984 \\
\hline 6 & 5.411800 & -2.870224 & 0.126058 \\
\hline 1 & 6.261714 & -2.677244 & 0.775796 \\
\hline 1 & 5.721252 & -2.668935 & -0.900676 \\
\hline 1 & 5.155812 & -3.927712 & 0.210552 \\
\hline 6 & 2.770552 & -2.785076 & -1.354448 \\
\hline 1 & 1.744984 & -3.149555 & -1.284894 \\
\hline 1 & 3.438763 & -3.639919 & -1.394628 \\
\hline 1 & 2.915912 & -2.162414 & -2.238675 \\
\hline 7 & -0.587822 & 1.944701 & -0.204449 \\
\hline 7 & -0.990840 & 0.634367 & 1.446930 \\
\hline 7 & 2.888377 & -0.531727 & 1.386847 \\
\hline 7 & 3.074111 & -2.002151 & -0.165021 \\
\hline 53 & 3.606035 & 1.547425 & -1.274930 \\
\hline 1 & 0.887620 & -0.319463 & -1.910887 \\
\hline 6 & -0.654333 & -2.148954 & -0.316893 \\
\hline 8 & -1.786736 & -1.701867 & -0.630667 \\
\hline 8 & -0.294765 & -3.246207 & 0.099332 \\
\hline 6 & -5.092920 & -1.048999 & -1.090860 \\
\hline 6 & -4.902079 & -0.017080 & 0.070296 \\
\hline
\end{tabular}




$\begin{array}{rrr}-3.796151 & -0.563304 & 0.806678 \\ -4.588084 & -2.257214 & -0.509571 \\ -6.092555 & 0.029024 & 1.022737 \\ -5.825134 & 0.629202 & 1.894023 \\ -6.969457 & 0.475061 & 0.551707 \\ -6.347919 & -0.974377 & 1.364399 \\ -4.553173 & 1.385813 & -0.395172 \\ -5.338687 & 1.781583 & -1.042643 \\ -4.458482 & 2.048423 & 0.468319 \\ -3.609849 & 1.387874 & -0.940659 \\ -6.538325 & -1.269651 & -1.501378 \\ -6.984036 & -0.337925 & -1.857259 \\ -6.572342 & -1.996450 & -2.312887 \\ -7.133418 & -1.654112 & -0.675403 \\ -4.249206 & -0.725742 & -2.320274 \\ -4.286531 & -1.581623 & -2.994087 \\ -4.637448 & 0.148878 & -2.845894 \\ -3.208225 & -0.564939 & -2.044831 \\ -3.684401 & -1.915238 & 0.466591 \\ -3.188844 & -2.710093 & 1.192832\end{array}$


Table S25

M06-2X/def2-TZVP

TS02

$1^{\text {st }}$ frequency: $-1130.5 \mathrm{~cm}^{-1}$

Sum of electronic and thermal Free Energies $=-1765.951111$ Hartree

\begin{tabular}{|c|c|c|c|}
\hline Atomic & \multicolumn{3}{|c|}{ Coordinates (Angstroms) } \\
\hline Number & $\mathrm{X}$ & Y & Z \\
\hline------ & ---------- & ---------- & ---------- \\
\hline 14 & -1.035795 & -0.112162 & -1.226415 \\
\hline 6 & -5.110565 & -1.049409 & -0.637824 \\
\hline 6 & -4.826436 & -0.248407 & 0.668292 \\
\hline 6 & -0.440976 & 1.479153 & -0.345350 \\
\hline 6 & 0.481275 & 3.460597 & 0.135202 \\
\hline 6 & 0.324637 & 2.787688 & 1.301483 \\
\hline 6 & 0.172156 & 2.907730 & -2.274675 \\
\hline 1 & -0.600930 & 2.403614 & -2.845105 \\
\hline 1 & 1.156981 & 2.537726 & -2.568823 \\
\hline 1 & 0.110488 & 3.978482 & -2.450672 \\
\hline 6 & 1.163818 & 4.745422 & -0.156485 \\
\hline 1 & 1.486429 & 5.210921 & 0.771700 \\
\hline 1 & 0.514774 & 5.449453 & -0.680294 \\
\hline 1 & 2.051925 & 4.561978 & -0.765788 \\
\hline 6 & 0.787264 & 3.109885 & 2.673802 \\
\hline 1 & 1.206623 & 4.112802 & 2.697201 \\
\hline 1 & 1.572712 & 2.410459 & 2.970455 \\
\hline 1 & -0.023388 & 3.064709 & 3.403146 \\
\hline 6 & -0.356210 & 0.452751 & 1.886860 \\
\hline 1 & -1.234844 & -0.140750 & 1.644936 \\
\hline 1 & -0.453341 & 0.816222 & 2.906525 \\
\hline 1 & 0.561890 & -0.133244 & 1.783573 \\
\hline 7 & -0.017358 & 2.643417 & -0.861319 \\
\hline 7 & -0.265209 & 1.582348 & 0.981362 \\
\hline 53 & 3.036630 & 0.932745 & -0.122412 \\
\hline 1 & -1.075344 & 0.289675 & -2.656872 \\
\hline 1 & -0.004919 & -1.466288 & -0.873581 \\
\hline 5 & -2.956152 & -0.306855 & -0.638531 \\
\hline 8 & -3.382346 & -0.170599 & 0.665838 \\
\hline 8 & -3.980718 & -0.693188 & -1.464354 \\
\hline 6 & -5.051320 & -2.558379 & -0.432519 \\
\hline 1 & -5.908226 & -2.916252 & 0.139515 \\
\hline 1 & -5.054873 & -3.043447 & -1.408155 \\
\hline 1 & -4.136155 & -2.845079 & 0.087649 \\
\hline 6 & -6.390860 & -0.663960 & -1.352474 \\
\hline 1 & -6.505948 & -1.275856 & -2.246977 \\
\hline 1 & -7.253794 & -0.836178 & -0.705596 \\
\hline 1 & -6.378775 & 0.381570 & -1.652878 \\
\hline 6 & -5.286354 & -0.927065 & 1.943497 \\
\hline 1 & -5.050337 & -0.294467 & 2.799368 \\
\hline 1 & -6.366999 & -1.082649 & 1.921552 \\
\hline 1 & -4.796655 & -1.888918 & 2.081173 \\
\hline 6 & -5.346944 & 1.182645 & 0.610911 \\
\hline 1 & -6.435836 & 1.211120 & 0.664781 \\
\hline 1 & -4.940759 & 1.737420 & 1.456436 \\
\hline 1 & -5.027474 & 1.675732 & -0.308024 \\
\hline 6 & 1.106784 & -2.463009 & -0.532822 \\
\hline 7 & 2.275132 & -2.396955 & -1.188796 \\
\hline 6 & 3.352826 & -2.667772 & -0.363158 \\
\hline 6 & 2.832201 & -2.930846 & 0.859493 \\
\hline 7 & 1.457210 & -2.794554 & 0.725268 \\
\hline 6 & 2.399500 & -1.932142 & -2.554012 \\
\hline 1 & 2.973667 & -1.004567 & -2.565307 \\
\hline 1 & 2.884499 & -2.687921 & -3.172375 \\
\hline 1 & 1.399554 & -1.741848 & -2.936057 \\
\hline
\end{tabular}




$\begin{array}{rrr}0.502955 & -3.009347 & 1.788449 \\ 0.449282 & -4.065115 & 2.058696 \\ 0.780784 & -2.433695 & 2.672065 \\ -0.473418 & -2.683147 & 1.435690 \\ 4.756333 & -2.549701 & -0.829069 \\ 4.939598 & -3.159696 & -1.715815 \\ 4.974105 & -1.504893 & -1.065971 \\ 5.442317 & -2.870182 & -0.047436 \\ 3.484394 & -3.218997 & 2.162052 \\ 3.371442 & -2.376075 & 2.848262 \\ 3.065003 & -4.107348 & 2.639237 \\ 4.549639 & -3.385746 & 2.016680\end{array}$


Table S26

M06-2X/def2-TZVP

TS03

$1^{\text {st }}$ frequency: $-54.1 \mathrm{~cm}^{-1}$

Sum of electronic and thermal Free Energies $=-1764.773519$ Hartree

\begin{tabular}{|c|c|c|c|}
\hline Atomic & \multicolumn{3}{|c|}{ Coordinates (Angstroms) } \\
\hline Number & $\mathrm{X}$ & Y & z \\
\hline 14 & -0.346311 & -0.641173 & 1.270599 \\
\hline 6 & 1.304546 & 1.490851 & 1.430365 \\
\hline 6 & 2.710055 & 2.800695 & 0.192391 \\
\hline 6 & 3.466716 & 1.841952 & 0.780791 \\
\hline 6 & 0.282872 & 3.397522 & 0.240590 \\
\hline 1 & 0.431465 & 4.418162 & 0.595521 \\
\hline 1 & -0.605591 & 2.975803 & 0.699733 \\
\hline 1 & 0.144629 & 3.409542 & -0.841675 \\
\hline 6 & 3.071529 & 3.918634 & -0.719014 \\
\hline 1 & 4.139309 & 3.903684 & -0.930570 \\
\hline 1 & 2.830722 & 4.888049 & -0.277603 \\
\hline 1 & 2.538772 & 3.853069 & -1.671266 \\
\hline 6 & 4.918560 & 1.538448 & 0.687605 \\
\hline 1 & 5.426326 & 2.286515 & 0.081389 \\
\hline 1 & 5.080605 & 0.559156 & 0.226396 \\
\hline 1 & 5.391270 & 1.524441 & 1.671591 \\
\hline 6 & 3.017001 & -0.002862 & 2.404442 \\
\hline 1 & 2.123473 & -0.514580 & 2.757639 \\
\hline 1 & 3.573750 & 0.388262 & 3.257299 \\
\hline 1 & 3.651527 & -0.700580 & 1.855726 \\
\hline 6 & 1.067361 & -0.931307 & -0.094971 \\
\hline 6 & 2.789056 & -0.712209 & -1.540261 \\
\hline 6 & 3.060383 & -1.777397 & -0.748598 \\
\hline 6 & 0.858288 & 0.808840 & -1.868901 \\
\hline 1 & -0.040677 & 1.085794 & -1.332605 \\
\hline 1 & 0.572648 & 0.425843 & -2.849859 \\
\hline 1 & 1.508286 & 1.674721 & -1.994599 \\
\hline 6 & 3.574042 & -0.066511 & -2.623293 \\
\hline 1 & 4.497321 & -0.614486 & -2.799682 \\
\hline 1 & 3.834105 & 0.960729 & -2.349933 \\
\hline 1 & 3.016173 & -0.031368 & -3.560710 \\
\hline 6 & 4.222943 & -2.703402 & -0.723083 \\
\hline 1 & 4.903618 & -2.469964 & -1.539125 \\
\hline 1 & 3.903030 & -3.740747 & -0.835224 \\
\hline 1 & 4.781215 & -2.628194 & 0.213404 \\
\hline 6 & 1.866596 & -2.977316 & 1.084942 \\
\hline 1 & 1.197735 & -2.663035 & 1.884286 \\
\hline 1 & 2.849034 & -3.215330 & 1.489927 \\
\hline 1 & 1.432193 & -3.851102 & 0.599475 \\
\hline 7 & 1.407389 & 2.566166 & 0.613858 \\
\hline 7 & 2.592602 & 1.079976 & 1.540459 \\
\hline 7 & 1.558330 & -0.225580 & -1.135125 \\
\hline 7 & 1.993703 & -1.893846 & 0.125353 \\
\hline 53 & -1.634022 & -2.762545 & -0.025350 \\
\hline 6 & -3.417238 & 1.433625 & -0.916542 \\
\hline 6 & -3.948985 & 1.387843 & 0.549157 \\
\hline 8 & -2.004843 & 1.186434 & -0.733536 \\
\hline
\end{tabular}




$\begin{array}{rrr}-2.993932 & 0.533254 & 1.197002 \\ -1.843952 & 0.474544 & 0.444554 \\ -3.596605 & 2.766068 & -1.618746 \\ -3.193064 & 2.701994 & -2.629807 \\ -4.656614 & 3.018566 & -1.691007 \\ -3.082658 & 3.568629 & -1.091667 \\ -3.953488 & 0.298466 & -1.778946 \\ -5.016275 & 0.427233 & -1.988335 \\ -3.409642 & 0.290318 & -2.723986 \\ -3.795203 & -0.665715 & -1.293176 \\ -3.890453 & 2.739562 & 1.251808 \\ -4.633407 & 3.431540 & 0.852348 \\ -4.086325 & 2.587735 & 2.312846 \\ -2.902245 & 3.190243 & 1.151474 \\ -5.332295 & 0.781494 & 0.698421 \\ -5.621226 & 0.795821 & 1.749319 \\ -6.067427 & 1.356366 & 0.130557 \\ -5.344810 & -0.251017 & 0.355808\end{array}$




\section{Table S27}

\section{M06-2X/def2-TZVP}

Compound 2a

$1^{\text {st }}$ frequency: $14.1 \mathrm{~cm}^{-1}$

Sum of electronic and thermal Free Energies $=-600.310083$ Hartree

\begin{tabular}{|c|c|c|c|}
\hline Atomic & \multicolumn{3}{|c|}{ Coordinates (Angstroms) } \\
\hline Number & $x$ & Y & z \\
\hline 6 & -1.293157 & -0.631900 & 0.237345 \\
\hline 6 & -0.897692 & 0.818228 & -0.200641 \\
\hline 8 & 0.391100 & 0.604592 & -0.823911 \\
\hline 8 & -0.001287 & -1.248253 & 0.457698 \\
\hline 6 & -0.663970 & 1.750526 & 0.980391 \\
\hline 1 & -0.208410 & 2.669867 & 0.613916 \\
\hline 1 & -1.601474 & 1.996135 & 1.480239 \\
\hline 1 & 0.018165 & 1.302612 & 1.704499 \\
\hline 6 & -1.835361 & 1.453210 & -1.206569 \\
\hline 1 & -2.839878 & 1.535739 & -0.786568 \\
\hline 1 & -1.482371 & 2.455336 & -1.449186 \\
\hline 1 & -1.883817 & 0.874234 & -2.126595 \\
\hline 6 & -2.099229 & -0.707616 & 1.517430 \\
\hline 1 & -3.043056 & -0.171067 & 1.401373 \\
\hline 1 & -2.323545 & -1.749754 & 1.744735 \\
\hline 1 & -1.552683 & -0.282512 & 2.356601 \\
\hline 6 & -1.974516 & -1.419671 & -0.872967 \\
\hline 1 & -2.041956 & -2.464549 & -0.570983 \\
\hline 1 & -2.980622 & -1.044245 & -1.061986 \\
\hline 1 & -1.401346 & -1.365924 & -1.799777 \\
\hline 5 & 0.893281 & -0.528187 & -0.266802 \\
\hline 8 & 2.198083 & -0.967716 & -0.473579 \\
\hline 6 & 3.232106 & -0.203787 & -0.095025 \\
\hline 8 & 3.149028 & 0.752213 & 0.611240 \\
\hline 1 & 4.170109 & -0.595568 & -0.503617 \\
\hline
\end{tabular}


Table S28

M06-2X/def2-TZVP

TS04

$1^{\text {st }}$ frequency: $-102.6 \mathrm{~cm}^{-1}$

Sum of electronic and thermal Free Energies $=-2365.094464$ Hartree

\begin{tabular}{|c|c|c|c|}
\hline Atomic & \multicolumn{3}{|c|}{ Coordinates (Angstroms) } \\
\hline Number & $\mathrm{X}$ & Y & z \\
\hline 14 & 0.470596 & -0.357794 & -0.704400 \\
\hline 6 & -0.487740 & -1.474080 & 0.511747 \\
\hline 6 & -1.660343 & -2.219667 & 2.258321 \\
\hline 6 & -2.011441 & -2.966472 & 1.175712 \\
\hline 6 & -0.091034 & -0.335868 & 2.709405 \\
\hline 1 & 0.811934 & 0.059998 & 2.249628 \\
\hline 1 & 0.183640 & -0.836779 & 3.635695 \\
\hline 1 & -0.785639 & 0.477854 & 2.908614 \\
\hline 6 & -2.166245 & -2.231967 & 3.654345 \\
\hline 1 & -2.961335 & -2.967505 & 3.754270 \\
\hline 1 & -2.570156 & -1.255130 & 3.927993 \\
\hline 1 & -1.380397 & -2.484885 & 4.367922 \\
\hline 6 & -3.048688 & -4.014570 & 1.004717 \\
\hline 1 & -3.606376 & -4.141809 & 1.930206 \\
\hline 1 & -2.617688 & -4.980651 & 0.736226 \\
\hline 1 & -3.742194 & -3.708277 & 0.218362 \\
\hline 6 & -1.336957 & -3.065840 & -1.219749 \\
\hline 1 & -2.179289 & -2.629422 & -1.752935 \\
\hline 1 & -1.463628 & -4.142598 & -1.132948 \\
\hline 1 & -0.422413 & -2.854145 & -1.767364 \\
\hline 6 & 2.147231 & -1.193898 & -1.050462 \\
\hline 6 & 4.063032 & -2.329557 & -0.967688 \\
\hline 6 & 4.195716 & -1.356453 & -1.908947 \\
\hline 6 & 2.293027 & -2.962695 & 0.680989 \\
\hline 1 & 1.300799 & -3.362635 & 0.486289 \\
\hline 1 & 2.283837 & -2.287364 & 1.539846 \\
\hline 1 & 2.968918 & -3.788383 & 0.880075 \\
\hline 6 & 5.054710 & -3.305399 & -0.448004 \\
\hline 1 & 5.987075 & -3.208847 & -0.998764 \\
\hline 1 & 4.709186 & -4.335157 & -0.553132 \\
\hline 1 & 5.264644 & -3.109738 & 0.605367 \\
\hline 6 & 5.359898 & -0.961317 & -2.741792 \\
\hline 1 & 6.212719 & -1.595304 & -2.512418 \\
\hline 1 & 5.643040 & 0.072229 & -2.536273 \\
\hline 1 & 5.144408 & -1.056109 & -3.807325 \\
\hline 6 & 2.724687 & 0.502109 & -2.749047 \\
\hline 1 & 1.693754 & 0.471401 & -3.100599 \\
\hline 1 & 3.384411 & 0.503985 & -3.611785 \\
\hline 1 & 2.899116 & 1.384734 & -2.133390 \\
\hline 7 & -0.718292 & -1.312792 & 1.824664 \\
\hline 7 & -1.258607 & -2.503717 & 0.123054 \\
\hline 7 & 2.778264 & -2.230218 & -0.475601 \\
\hline 7 & 2.991962 & -0.692782 & -1.957363 \\
\hline 53 & 3.532008 & 0.523685 & 1.506109 \\
\hline 6 & 0.010119 & 3.484126 & 0.965424 \\
\hline 6 & 0.531414 & 3.781871 & -0.475738 \\
\hline 8 & -0.226175 & 2.050299 & 0.912024 \\
\hline
\end{tabular}




$\begin{array}{rrr}1.135668 & 2.519054 & -0.855678 \\ 0.555124 & 1.551926 & -0.089275 \\ -1.293570 & 4.173307 & 1.315414 \\ -1.583732 & 3.906179 & 2.332148 \\ -1.170085 & 5.257381 & 1.270741 \\ -2.093582 & 3.879747 & 0.638634 \\ 1.056822 & 3.720415 & 2.043600 \\ 1.251371 & 4.786160 & 2.171717 \\ 0.683514 & 3.321679 & 2.987843 \\ 1.989412 & 3.205504 & 1.809031 \\ -0.591189 & 4.032598 & -1.474095 \\ -1.085787 & 4.985965 & -1.283919 \\ -0.168192 & 4.053800 & -2.478196 \\ -1.331442 & 3.230458 & -1.437450 \\ 1.584476 & 4.868452 & -0.550842 \\ 1.881984 & 5.014601 & -1.589290 \\ 1.182471 & 5.811532 & -0.174631 \\ 2.466076 & 4.601093 & 0.028422 \\ -0.694794 & -0.357941 & -2.246054 \\ -1.780256 & 0.114548 & -1.812508 \\ -0.374595 & -0.767571 & -3.355849 \\ -4.907207 & 0.873647 & -0.756286 \\ -4.843236 & -0.489285 & 0.009821 \\ -3.912968 & -1.255864 & -0.771198 \\ -4.579341 & 0.486201 & -2.095412 \\ -6.168943 & -1.243765 & -0.013288 \\ -6.007326 & -2.249843 & 0.377102 \\ -6.925249 & -0.751466 & 0.599559 \\ -6.542862 & -1.329645 & -1.033871 \\ -4.333491 & -0.377898 & 1.435950 \\ -4.962364 & 0.301110 & 2.016088 \\ -4.363910 & -1.360076 & 1.912831 \\ -3.306261 & -0.010745 & 1.451964 \\ -6.871 .865406 & 1.434650 & -0.258378 \\ -6.6158649 & -0.666750 & -2.035819 \\ -6.226094 & -1.526629 & -0.758783 \\ -7.021243 & 0.893119 & -1.240027 \\ -3.861348 & 1.876833 & -0.276869 \\ -3.847072 & 2.711643 & -0.979614 \\ -1.278885 & -2.999215\end{array}$


Table S29

M06-2X/def2-TZVP

TS05

1st frequency: $-1306.7 \mathrm{~cm}-1$

Sum of electronic and thermal Free Energies $=-1542.825339$ Hartree

\begin{tabular}{|c|c|c|c|}
\hline \multirow{2}{*}{$\begin{array}{l}\text { Atomic } \\
\text { Number }\end{array}$} & \multicolumn{3}{|c|}{ Coordinates (Angstroms) } \\
\hline & $\mathrm{X}$ & Y & Z \\
\hline & --- & - & 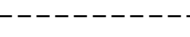 \\
\hline 14 & -0.555410 & 0.773145 & -0.149166 \\
\hline 6 & -2.331325 & 0.001454 & -0.088827 \\
\hline 6 & -4.577368 & 0.056742 & -0.207275 \\
\hline 6 & -4.207703 & -1.243835 & -0.092182 \\
\hline 6 & -3.336191 & 2.250918 & -0.313702 \\
\hline 1 & -4.307027 & 2.633522 & -0.612668 \\
\hline 1 & -2.595514 & 2.521937 & -1.064606 \\
\hline 1 & -3.036553 & 2.679136 & 0.642212 \\
\hline 6 & -5.925352 & 0.674744 & -0.311612 \\
\hline 1 & -6.692347 & -0.093006 & -0.244460 \\
\hline 1 & -6.054307 & 1.196955 & -1.261155 \\
\hline 1 & -6.095107 & 1.390776 & 0.493842 \\
\hline 6 & -5.032549 & -2.479981 & -0.040520 \\
\hline 1 & -6.081321 & -2.227075 & -0.176302 \\
\hline 1 & -4.935966 & -2.991794 & 0.918882 \\
\hline 1 & -4.754322 & -3.181259 & -0.828992 \\
\hline 6 & -2.024941 & -2.456776 & 0.164524 \\
\hline 1 & -2.684336 & -3.274371 & 0.439744 \\
\hline 1 & -1.299461 & -2.300527 & 0.959186 \\
\hline 1 & -1.497963 & -2.718107 & -0.752240 \\
\hline 6 & 0.576362 & -0.711248 & 0.215988 \\
\hline 6 & 2.172353 & -1.995252 & 1.094826 \\
\hline 6 & 2.026784 & -2.355415 & -0.207134 \\
\hline 6 & 1.246912 & -0.186586 & 2.543381 \\
\hline 1 & 0.281573 & 0.295686 & 2.659475 \\
\hline 1 & 2.010964 & 0.584437 & 2.415700 \\
\hline 1 & 1.470351 & -0.814552 & 3.402288 \\
\hline 6 & 3.166546 & -2.418724 & 2.112405 \\
\hline 1 & 3.810140 & -3.194312 & 1.704308 \\
\hline 1 & 2.690647 & -2.810569 & 3.012815 \\
\hline 1 & 3.794002 & -1.570053 & 2.392427 \\
\hline 6 & 2.815561 & -3.288197 & -1.050473 \\
\hline 1 & 3.549023 & -3.809995 & -0.440660 \\
\hline 1 & 3.352389 & -2.730388 & -1.820950 \\
\hline 1 & 2.186205 & -4.036565 & -1.535552 \\
\hline 6 & 0.736203 & -1.424729 & -2.135092 \\
\hline 1 & -0.302207 & -1.134091 & -2.283901 \\
\hline 1 & 0.918359 & -2.369021 & -2.642174 \\
\hline 1 & 1.393329 & -0.640883 & -2.524229 \\
\hline 7 & -3.410460 & 0.797703 & -0.213911 \\
\hline 7 & -2.821716 & -1.254051 & -0.018560 \\
\hline 7 & 1.238647 & -1.009457 & 1.343084 \\
\hline 7 & 1.008904 & -1.576683 & -0.717195 \\
\hline 53 & 3.028821 & 1.224142 & -0.619703 \\
\hline 8 & -0.630737 & 1.758491 & 1.155426 \\
\hline 6 & -0.361672 & 3.115607 & 0.426837 \\
\hline 8 & -0.536817 & 4.089656 & 1.045631 \\
\hline 1 & -0.301245 & 2.170495 & -0.949651 \\
\hline
\end{tabular}


Table S30

M06-2X/def2-TZVP

Int05

Sum of electronic and thermal Free Energies $=-1542.909572$ Hartree

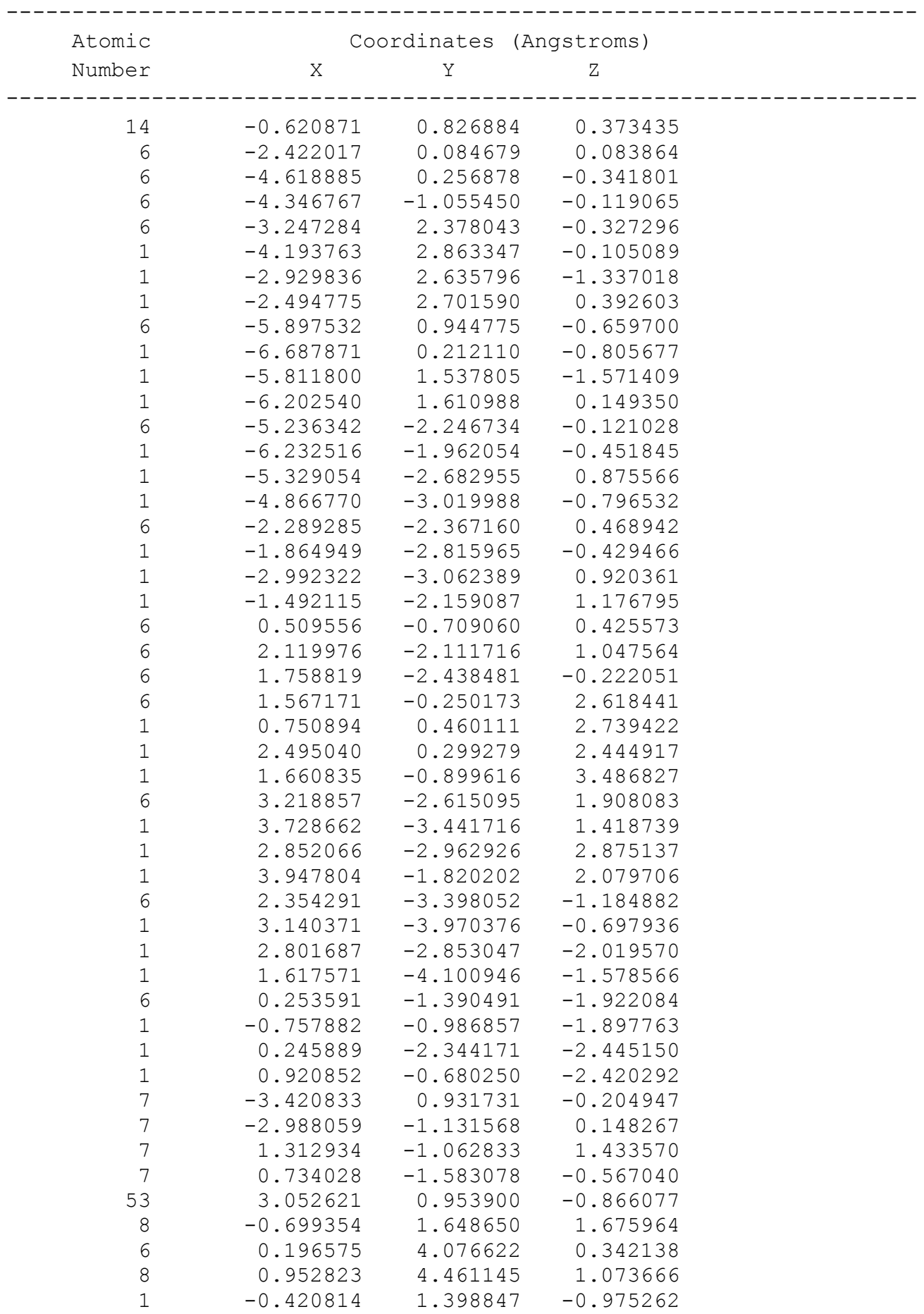


Table S31

M06-2X/def2-TZVP

Int06

$1^{\text {st }}$ frequency: $9.1 \mathrm{~cm}^{-1}$

Sum of electronic and thermal Free Energies $=-1954.587092$ Hartree

\begin{tabular}{|c|c|c|c|}
\hline \multirow{2}{*}{$\begin{array}{l}\text { Atomic } \\
\text { Number }\end{array}$} & \multicolumn{3}{|c|}{ Coordinates (Angstroms) } \\
\hline & $\mathrm{X}$ & Y & $\mathrm{Z}$ \\
\hline-------- & ----------1 & ----------- & ----------1 \\
\hline 14 & 0.388794 & -0.225724 & -0.630415 \\
\hline 6 & 0.281967 & 1.554744 & 0.035331 \\
\hline 6 & 0.320812 & 3.783420 & 0.146192 \\
\hline 6 & -0.360492 & 3.322731 & 1.232129 \\
\hline 6 & 1.426616 & 2.741378 & -1.844195 \\
\hline 1 & 2.050581 & 1.856451 & -1.961505 \\
\hline 1 & 2.082122 & 3.607781 & -1.824874 \\
\hline 1 & 0.713811 & 2.834942 & -2.663160 \\
\hline 6 & 0.638561 & 5.170004 & -0.284372 \\
\hline 1 & 0.178432 & 5.883344 & 0.395519 \\
\hline 1 & 0.262317 & 5.366526 & -1.289426 \\
\hline 1 & 1.714896 & 5.350047 & -0.285847 \\
\hline 6 & -1.023122 & 4.043083 & 2.349605 \\
\hline 1 & -0.944162 & 5.117450 & 2.200960 \\
\hline 1 & -0.564457 & 3.799520 & 3.309593 \\
\hline 1 & -2.081072 & 3.782198 & 2.407180 \\
\hline 6 & -1.027601 & 1.083372 & 2.112741 \\
\hline 1 & -2.102311 & 1.255767 & 2.071392 \\
\hline 1 & -0.646343 & 1.324995 & 3.105682 \\
\hline 1 & -0.861173 & 0.028458 & 1.869740 \\
\hline 6 & 1.654966 & -1.178637 & 0.421066 \\
\hline 6 & 3.285319 & -1.889799 & 1.757938 \\
\hline 6 & 2.907208 & -2.935155 & 0.974865 \\
\hline 6 & 2.634725 & 0.521931 & 1.933877 \\
\hline 1 & 1.688014 & 0.882765 & 2.329055 \\
\hline 1 & 3.008884 & 1.161679 & 1.131986 \\
\hline 1 & 3.363832 & 0.501284 & 2.737586 \\
\hline 6 & 4.378019 & -1.786460 & 2.758920 \\
\hline 1 & 4.861630 & -2.753526 & 2.874476 \\
\hline 1 & 4.006168 & -1.478982 & 3.737709 \\
\hline 1 & 5.132040 & -1.069800 & 2.427195 \\
\hline 6 & 3.430726 & -4.322765 & 0.889960 \\
\hline 1 & 4.203996 & -4.471670 & 1.639695 \\
\hline 1 & 3.873283 & -4.512312 & -0.089674 \\
\hline 1 & 2.645273 & -5.059564 & 1.065060 \\
\hline 6 & 1.132549 & -3.252158 & -0.792075 \\
\hline 1 & 0.118682 & -3.382537 & -0.410797 \\
\hline 1 & 1.620068 & -4.211755 & -0.932366 \\
\hline 1 & 1.121666 & -2.731666 & -1.750571 \\
\hline 7 & 0.707427 & 2.674009 & -0.574252 \\
\hline 7 & -0.371912 & 1.949685 & 1.137249 \\
\hline 7 & 2.475114 & -0.825917 & 1.417492 \\
\hline 7 & 1.891645 & -2.469006 & 0.170839 \\
\hline 53 & 4.243274 & 0.216839 & -1.379736 \\
\hline 1 & 0.937504 & -0.102427 & -1.992664 \\
\hline 6 & -1.277682 & -1.132955 & -0.303206 \\
\hline 8 & -2.264575 & 0.018918 & -0.340492 \\
\hline 8 & -1.208543 & -1.727184 & 0.848476 \\
\hline 6 & -5.518837 & -1.356214 & -0.823994 \\
\hline 6 & -5.781082 & -0.133409 & 0.113729 \\
\hline
\end{tabular}




$\begin{array}{lrr}-4.555526 & 0.607923 & -0.002698 \\ -4.098095 & -1.522068 & -0.716078 \\ -5.921395 & -0.544576 & 1.575302 \\ -5.903465 & 0.353918 & 2.192860 \\ -6.859367 & -1.071272 & 1.755684 \\ -5.091473 & -1.186902 & 1.875463 \\ -6.944088 & 0.744952 & -0.303759 \\ -7.870788 & 0.167296 & -0.321183 \\ -7.062411 & 1.558135 & 0.413050 \\ -6.780230 & 1.177475 & -1.288848 \\ -6.205248 & -2.639448 & -0.397029 \\ -7.287861 & -2.499290 & -0.356893 \\ -5.988666 & -3.425911 & -1.120472 \\ -5.852691 & -2.968349 & 0.578387 \\ -5.831074 & -1.048790 & -2.284236 \\ -5.432318 & -1.853171 & -2.902174 \\ -6.905528 & -0.974693 & -2.457209 \\ -5.359955 & -0.113764 & -2.591843 \\ -3.570853 & -0.302627 & -0.348375 \\ -1.578369 & -1.755245 & -1.170782\end{array}$




\section{Table S32}

\section{M06-2X/def2-TZVP}

TS06

$1^{\text {st }}$ frequency: $-168.3 \mathrm{~cm}^{-1}$

Sum of electronic and thermal Free Energies $=-1954.592477$ Hartree

\begin{tabular}{|c|c|c|c|}
\hline \multirow{2}{*}{$\begin{array}{l}\text { Atomic } \\
\text { Number }\end{array}$} & \multicolumn{3}{|c|}{ Coordinates (Angstroms) } \\
\hline & $\mathrm{X}$ & Y & $\mathrm{Z}$ \\
\hline 14 & -0.174655 & -1.444939 & -0.376807 \\
\hline 6 & -0.287240 & 0.411666 & 0.123362 \\
\hline 6 & -0.464267 & 2.642478 & 0.174094 \\
\hline 6 & -0.382150 & 2.203659 & 1.456708 \\
\hline 6 & -0.297038 & 1.612552 & -2.063583 \\
\hline 1 & 0.705078 & 1.979408 & -2.295851 \\
\hline 1 & -1.048317 & 2.295610 & -2.456114 \\
\hline 1 & -0.451347 & 0.631758 & -2.498874 \\
\hline 6 & -0.457639 & 4.016054 & -0.386215 \\
\hline 1 & -0.498678 & 4.745117 & 0.419238 \\
\hline 1 & -1.306222 & 4.194379 & -1.049042 \\
\hline 1 & 0.468156 & 4.180763 & -0.943186 \\
\hline 6 & -0.290169 & 2.950415 & 2.735008 \\
\hline 1 & -0.385124 & 4.017463 & 2.549635 \\
\hline 1 & 0.681134 & 2.774493 & 3.202054 \\
\hline 1 & -1.073163 & 2.653319 & 3.434033 \\
\hline 6 & -0.047688 & 0.020030 & 2.577002 \\
\hline 1 & -0.770115 & 0.279655 & 3.347450 \\
\hline 1 & 0.966636 & 0.224987 & 2.923473 \\
\hline 1 & -0.175580 & -1.034738 & 2.352799 \\
\hline 6 & 1.739541 & -1.680117 & -0.532467 \\
\hline 6 & 3.857983 & -2.057283 & 0.077297 \\
\hline 6 & 3.875611 & -1.580685 & -1.194542 \\
\hline 6 & 2.109442 & -2.568156 & 1.770187 \\
\hline 1 & 2.641424 & -3.483167 & 2.023988 \\
\hline 1 & 1.038689 & -2.784472 & 1.783393 \\
\hline 1 & 2.358057 & -1.796476 & 2.499140 \\
\hline 6 & 4.966078 & -2.382463 & 1.009158 \\
\hline 1 & 5.922156 & -2.231742 & 0.514081 \\
\hline 1 & 4.919550 & -3.416966 & 1.353027 \\
\hline 1 & 4.931725 & -1.726424 & 1.880964 \\
\hline 6 & 5.006662 & -1.183141 & -2.068020 \\
\hline 1 & 5.950393 & -1.405759 & -1.576228 \\
\hline 1 & 4.964664 & -0.105527 & -2.245792 \\
\hline 1 & 4.991171 & -1.705310 & -3.026117 \\
\hline 6 & 2.197753 & -0.729860 & -2.795248 \\
\hline 1 & 1.148320 & -0.907833 & -3.001225 \\
\hline 1 & 2.793179 & -1.141047 & -3.607356 \\
\hline 1 & 2.395476 & 0.339128 & -2.688525 \\
\hline 7 & -0.425962 & 1.522335 & -0.624456 \\
\hline 7 & -0.293702 & 0.833452 & 1.397175 \\
\hline 7 & 2.535910 & -2.128714 & 0.449910 \\
\hline 7 & 2.562667 & -1.386009 & -1.556164 \\
\hline 53 & 3.044067 & 1.686308 & 0.090466 \\
\hline 1 & -0.577406 & -1.410200 & -1.807285 \\
\hline 6 & -1.461977 & -2.655100 & 1.017849 \\
\hline 8 & -2.351774 & -1.582253 & 1.454820 \\
\hline 8 & -0.850794 & -3.219228 & 1.938734 \\
\hline 6 & -4.403814 & -0.489249 & -1.249414 \\
\hline 6 & -5.148784 & 0.054937 & 0.013353 \\
\hline 8 & -4.142998 & -0.055288 & 1.034502 \\
\hline
\end{tabular}




$\begin{array}{rrr}-3.474509 & -1.428103 & -0.676275 \\ -6.316787 & -0.832052 & 0.429064 \\ -6.659341 & -0.517101 & 1.414231 \\ -7.147372 & -0.752810 & -0.273247 \\ -6.009922 & -1.877104 & 0.492632 \\ -5.595924 & 1.500243 & -0.091310 \\ -6.282545 & 1.628356 & -0.930703 \\ -6.116305 & 1.785533 & 0.822877 \\ -4.747124 & 2.168792 & -0.222704 \\ -5.287691 & -1.208414 & -2.249126 \\ -6.065628 & -0.538026 & -2.620188 \\ -4.686697 & -1.538535 & -3.096706 \\ -5.758691 & -2.081917 & -1.803389 \\ -3.578115 & 0.585306 & -1.945913 \\ -2.918062 & 0.101125 & -2.667061 \\ -4.212181 & 1.298573 & -2.474212 \\ -2.966571 & 1.126536 & -1.221232 \\ -3.283337 & -1.055097 & 0.641120 \\ -1.987624 & -3.232931 & 0.244068\end{array}$




\section{S4. References}

(1) Li, Y.; Chan, Y.-C.; Leong, B.-X.; Li, Y.; Richards, E.; Purushothaman, I.; De, S.; Parameswaran, P.; So, C.-W., Trapping a Silicon(I) Radical with Carbenes: A Cationic cAAC-Silicon(I) Radical and an NHC-Parent-Silyliumylidene Cation. Angew. Chem. Int. Ed. 2017, 56, 7573-7578.

(2) (a) Bontemps, S.; Vendier, L.; Sabo-Etienne, S., Borane-Mediated Carbon Dioxide Reduction at Ruthenium: Formation of $\mathrm{C} 1$ and $\mathrm{C} 2$ Compounds. Angew. Chem. Int. Ed. 2012, 51, 1671-1674.

(b) Bontemps, S.; Sabo-Etienne, S., Trapping Formaldehyde in the Homogeneous Catalytic Reduction of Carbon Dioxide. Angew. Chem. Int. Ed. 2013, 52, 10253-10255.

(3) (a) Courtemanche, M.-A.; Légaré, M.-A.; Maron, L.; Fontaine, F.-G., A Highly Active Phosphine-Borane Organocatalyst for the Reduction of $\mathrm{CO}_{2}$ to Methanol Using Hydroboranes. J. Am. Chem. Soc. 2013, 135, 9326-9329. (b) Povie, G.; Villa, G.; Ford, L.; Pozzi, D.; Schiesser, C. H.; Renaud, P., Role of Catechol in the Radical Reduction of B-alkylcatecholboranes in Presence of Methanol. Chem. Commun. 2010, 46, 803-805. (c) Ho, S. Y. F.; So, C.-W.; SaffonMerceron, N.; Mézailles, N., Formation of a Zwitterionic Boronium Species from the Reaction of a Stable Carbenoid with Borane: $\mathrm{CO}_{2}$ Reduction. Chem. Commun. 2015, 51, 2107-2110.

(4) Chakraborty, S.; Zhang, J.; Krause, J. A.; Guan, H., An Efficient Nickel Catalyst for the Reduction of Carbon Dioxide with a Borane. J. Am. Chem. Soc. 2010, 132, 8872-8873.

(5) (a) Rao, B.; Chong, C. C.; Kinjo, R., Metal-Free Regio- and Chemoselective Hydroboration of Pyridines Catalysed by 1,3,2-Diazaphosphenium Triflate. J. Am. Chem. Soc. 2018, 140, 652-656.

(b) Mukherjee, D.; Shirase, S.; Spaniol, T. P.; Mashima, K.; Okuda, J., Magnesium Hydridotriphenylborate $\left[\mathrm{Mg}(\mathrm{thf})_{6}\right]\left[\mathrm{HBPh}_{3}\right]_{2}$ : a Versatile Hydroboration Catalyst. Chem. Commun. 2016, 52, 13155-13158. (c) Lawson, J. R.; Wilkins, L. C.; Melen, R. L., Tris(2,4,6trifluorophenyl)borane: An Efficient Hydroboration Catalyst. Chem. -Eur. J. 2017, 23, 1099711000 .

(6) Sheldrick, G. M. SADABS V2014/4 (Bruker AXS Inc.) University of Göttingen, Göttingen, Germany, 2014.

(7) SHELXL-2014/6 (Sheldrick, 2014); Bruker AXS Inc., Madison, WI, USA, 2014.

(8) Frisch, M. J.; Trucks, G. W.; Schlegel, H. B.; Scuseria, G. E.; Robb, M. A.; Cheeseman, J. R.; Scalmani, G.; Barone, V.; Mennucci, B.; Petersson, G. A. et al. Gaussian, Inc., Wallingford CT, 2016.

(9) Zhang, C.; Patschinski, P.; Stephenson, D. S.; Panisch, R.; Wender, J. H.; Holthausen, M. C.; Zipse, H., The Calculation of ${ }^{29} \mathrm{Si}$ NMR Chemical Shifts of Tetracoordinated Silicon Compounds in the Gas Phase and in Solution. PCCP 2014, 16, 16642-16650.

(10) Lu, T.; Chen, F., Multiwfn: A Multifunctional Wavefunction Analyzer. J. Comput. Chem. 2012, 33, 580-592.

(11) Kajiwara, T.; Takeda, N.; Sasamori, T.; Tokitoh, N., Insertion of an Overcrowded Silylene into Hydro- and Haloboranes: A Novel Synthesis of Silylborane Derivatives and Their Properties. Organometallics 2004, 23, 4723-4734.

(12) Ghadwal, R. S.; Roesky, H. W.; Merkel, S.; Henn, J.; Stalke, D., Lewis Base Stabilized Dichlorosilylene. Angew. Chem. Int. Ed. 2009, 48, 5683-5686.

(13) Arduengo, A. J.; Harlow, R. L.; Kline, M., A Stable Crystalline Carbene. J. Am. Chem. Soc. 1991, $113,361-363$. 